\title{
Einfluss der Pflanzendichte und -architektur auf Abundanz und innerpflanzliche Verteilung stängelminierender Schadinsekten in Winterraps
}

\author{
Dissertation \\ zur Erlangung des Doktorgrades \\ der Fakultät für Agrarwissenschaften \\ der Georg-August-Universität Göttingen
}

vorgelegt von

Helene Nuss

geboren in Leninabad

Göttingen, Juli 2004 


\section{D7}

Referent: Prof. Dr. S. Vidal

Korreferent: Prof. Dr. A. von Tiedemann

Tag der mündlichen Prüfung: 15. Juli 2004 


\section{Inhaltsverzeichnis}

2 Material und Methoden 5

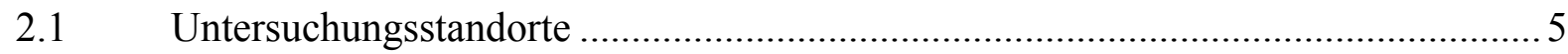

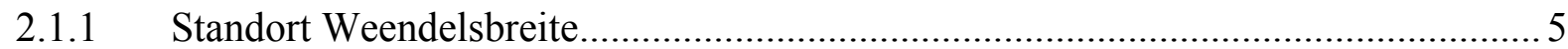

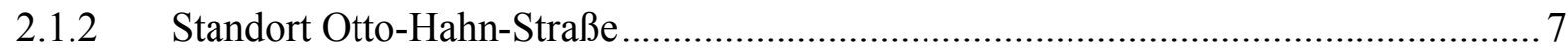

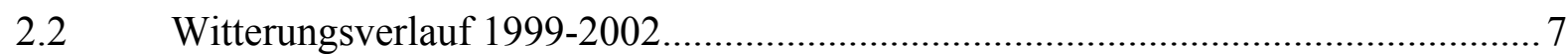

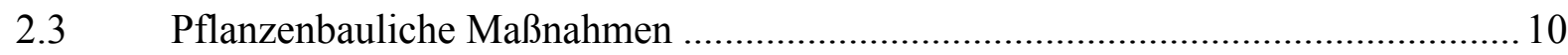

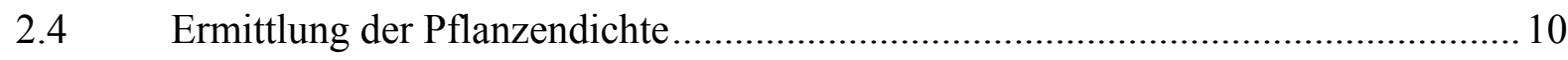

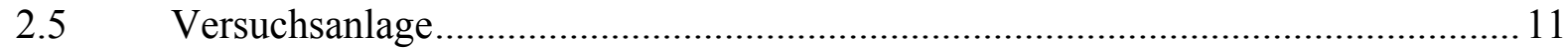

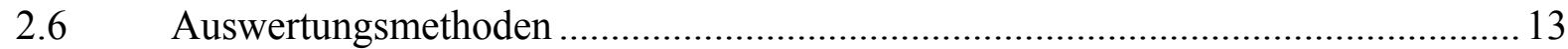

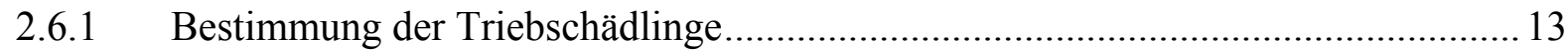

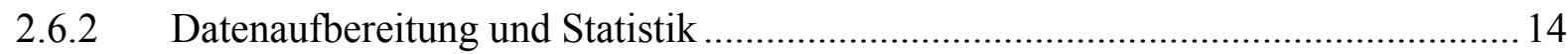

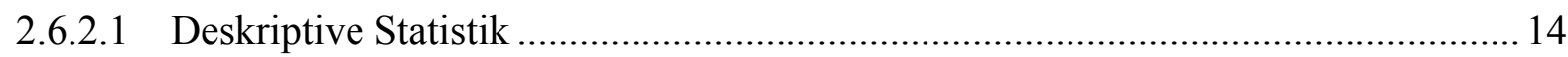

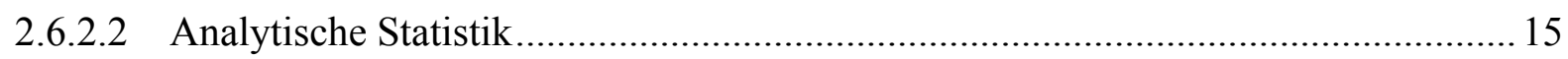

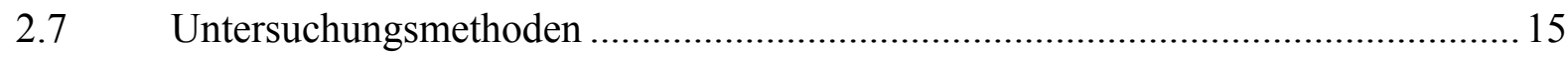

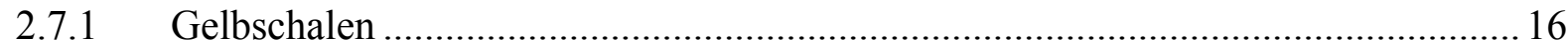

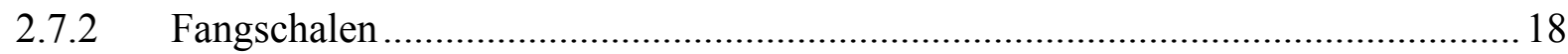

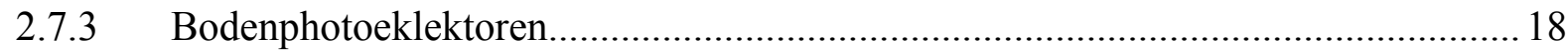

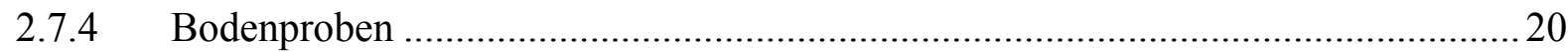

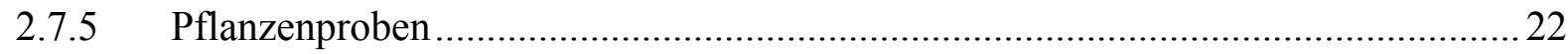

2.7.6 Ermittlung der Pflanzenlänge im Bestand ........................................................... 25

2.7.7 Ermittlung der Biomasse der Rapspflanzen im Labor ........................................ 25

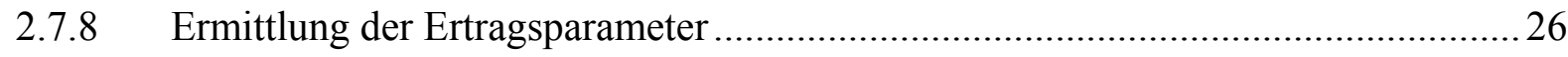

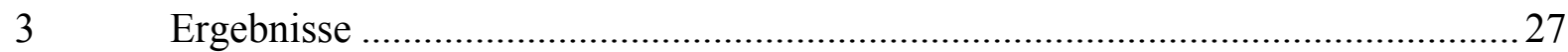

3.1 Entwicklung der Pflanzendichten und Wachstumsparameter der Pflanzen

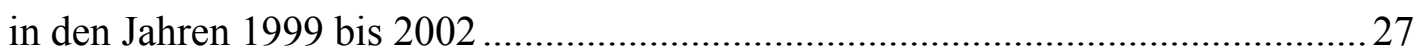

3.1.1 Vorwinterentwicklung der Pflanzen in Anhängigkeit von der Witterung .............. 27

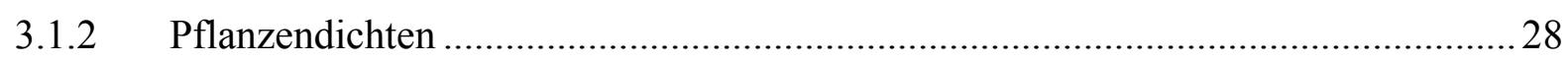

3.1.3 Absterben der Triebe während des Winters ........................................................29

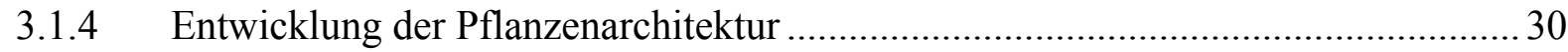


3.1.4.1 Sprossdurchmesser, Anzahl der Blätter und Seitentriebe sowie Sprosslänge.......... 30

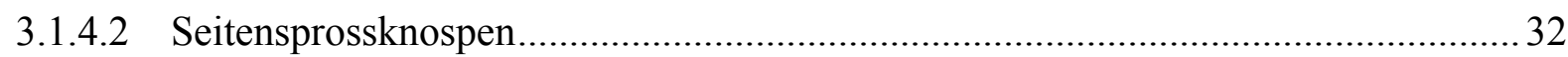

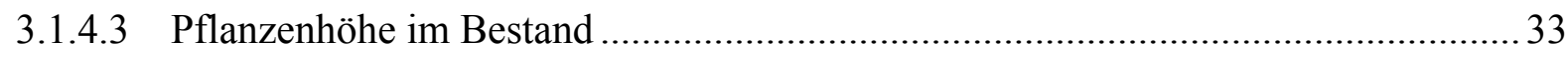

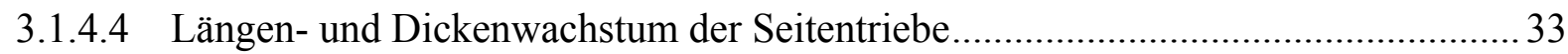

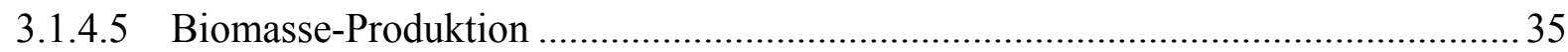

3.1.5 Beziehung zwischen den Wachstumsparametern der Pflanzen und

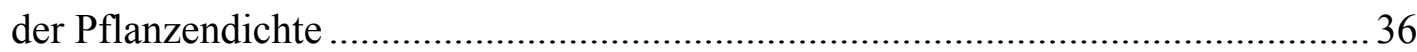

3.2 Befallsentwicklung von P. chrysocephala in Abhängigkeit von Pflanzendichte und -architektur in den Jahren 1999 bis 2001 ........................................................ 38

3.2.1 Zuwanderung von $P$. chrysocephala in die Winterrapsfelder ................................ 38

3.2.2 Besiedlungsdichte und Geschlechterverhältnis der Imagines von

P. chrysocephala

3.2.3 Aktivitätsdichte und Geschlechterverhältnis der Imagines von

P. chrysocephala

3.2.4 Abundanz und Verteilung der Eier von P. chrysocephala .................................... 44

3.2.5 Abundanz der Larven von P. chrysocephala ...................................................... 45

3.2.6 Beziehung zwischen der Larvenzahl/Pflanze von P. chrysocephala und der Pflanzendichte 46

3.2.7 Beziehung zwischen der Larvenzahl/Pflanze von P. chrysocephala und der Pflanzenarchitektur.

3.2.8 Larvendichte von P. chrysocephala in Bezug auf die Biomasse der Pflanzen ........ 48

3.2.9 Räumliche Verteilung der Eier und Larven von P. chrysocephala in den

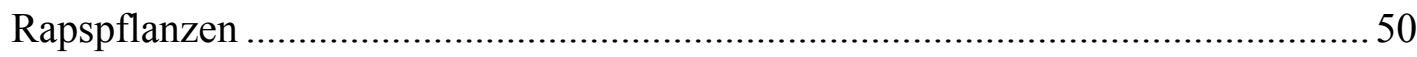

3.2.9.1 Vertikale Verteilung der Larven von P. chrysocephala auf unterschiedliche Blattetagen der Hauptsprossachse 50

3.2.9.2 Verteilung der Larven von P. chrysocephala in Endknospe, Seitentriebknospen, Sprossachse und Blättern.

3.3 Befallsentwicklung von C. napi und C. pallidactylus in Abhängigkeit von

Pflanzendichte und -architektur in den Jahren 2000 bis 2002 .................................59

3.3.1 Aktivitätsbeginn von $C$. napi auf den Überwinterungsfeldern................................59

3.3.2 Einwanderung von C. napi und C. pallidactylus in die Winterrapsfelder ............... 61

3.3.3 Abundanz der Eigelege und Larven von C. napi und C. pallidactylus .................... 64

3.3.4 Wundkallusbildung der Rapspflanzen als Reaktion auf die Eiablage von C. pallidactylus 
3.3.5 Beziehung zwischen der Anzahl der Eigelege/Pflanze von C. napi und der Pflanzendichte

3.3.6 Beziehung zwischen der Anzahl der Eigelege/Pflanze von C. napi und der Pflanzenarchitektur. 70

3.3.7 Beziehung zwischen der Anzahl der Eigelege/Pflanze von C. pallidactylus und der Pflanzendichte

3.3.8 Beziehung zwischen der Anzahl der Eigelege/Pflanze von C. pallidactylus und der Pflanzenarchitektur.

3.3.9 Räumliche Verteilung der Eier und Larven von C. napi und C. pallidactylus in den Rapspflanzen

3.3.9.1 Vertikale Verteilung der Eier von C. napi auf verschiedene Abschnitte des Haupttriebes. .74

3.3.9.2 Verteilung der Eier und Larven von C. napi auf Haupt- und Seitentriebe .75

3.3.9.3 Verteilung der Eigelege und Larven von C. pallidactylus auf Blätter und Triebe.

3.3.9.4 Vertikale Verteilung der Eigelege von C. pallidactylus auf unterschiedliche Blattetagen des Haupttriebes .....

3.3.9.5 Verteilung der Eigelege von C. pallidactylus auf das basale, mittlere und apikale Drittel der Blätter .

3.3.9.6 Häufigkeitsverteilung unterschiedlich großer Eigelege von C. pallidactylus an den Blättern der Haupttriebe .

3.3.9.7 Verteilung und Größe der Eigelege von C. pallidactylus in den Blattstielen, in dem an den Blattstiel angrenzenden Bereich der basalen Blattfiedern und der Blattspreitenbasis sowie in den Verzweigungen der Blattadern

3.3.10 Verteilung verschiedener Larvenstadien von C. napi und C. pallidactylus auf Haupt- und Seitentriebe. .86

3.3.10.1 Altersstruktur der Larven von C. napi und C. pallidactylus . .86

3.3.10.2 Verteilung verschiedener Larvenstadien von C. napi auf Haupt- und Seitentriebe

3.3.10.3 Verteilung verschiedener Larvenstadien von C. pallidactylus auf Haupt- und Seitentriebe

3.3.10.4 Einfluss des Stängelbasisdurchmessers der Rapspflanzen auf die Eiablage von C. napi und C. pallidactylus 
3.4 Kornertrag in Abhängigkeit von Pflanzendichte und -architektur .........................95

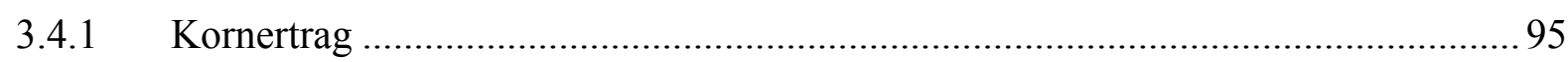

3.4.2 Beziehung zwischen den Ertragsparametern der Pflanzen und der Pflanzen-

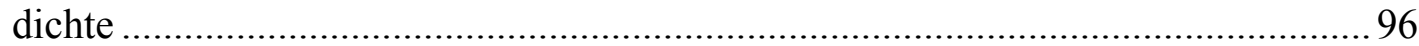

3.4.3 Beziehung zwischen den Ertragsparametern der Pflanzen und der Pflanzenarchitektur.

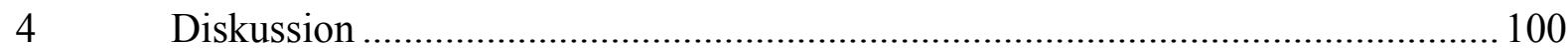

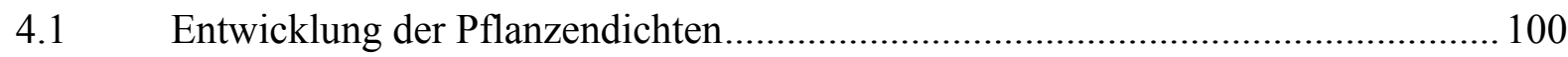

4.2 Einfluss der Pflanzendichte auf die Wachstumsparameter der Pflanzen ............... 102

4.3 Beurteilung der Methoden zur Erfassung der Triebschädlinge ............................. 104

4.4 Phänologie der Triebschädlinge P. chrysocephala, C. napi und

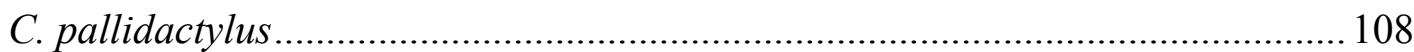

4.5 Einfluss der Pflanzendichte und -architektur auf die Befallsdichte von

P. chrysocephala .

4.6 Einfluss der Pflanzendichte und -architektur auf die innerpflanzliche

Verteilung und Schadwirkung von $P$. chrysocephala .....

4.7 Einfluss der Pflanzendichte und-architektur auf die Befallsdichte von

C. napi und C. pallidactylus

4.8 Einfluss der Pflanzendichte und -architektur auf die innerpflanzliche

Verteilung und Schadwirkung von C. napi und C. pallidactylus.

4.9 Einfluss der Pflanzendichte und -architektur auf den Kornertrag ....

$5 \quad$ Zusammenfassung. 126

6 Literatur 129

Anhang 


\section{Abkürzungsverzeichnis}

Tab. A Tabelle im Anhang

$\mathrm{Abb}$

Abbildung

$\mathrm{B}$

Bestimmtheitsmaß (in \%)

b

Regressionskoeffizient der Regressionsgeraden $y=a+b x$

$\mathrm{BBCH} \quad$ Codierung der phänologischen Entwicklungsstadien von Winterraps

$c v$

Variationskoeffizient (in \%)

Ek

Knospenblätter der Endknospe

$\mathrm{F}$

F-Wert

Ind.

Individuen

Kap.

Kapitel

$\mathrm{Kö.} / \mathrm{m}^{2}$

Körner/m²

L1

1. Larvenstadium

L2

2. Larvenstadium

L3

3. Larvenstadium

$\mathrm{NN}$

Normal Null

n.s.

nicht signifikant mit einer Fehlerwahrscheinlichkeit von über $5 \%$

$\mathrm{p}$

Signifikanzniveau

Pfl.

Pflanzlich, Pflanze

r

Korrelationskoeffizient

SD

engl. standard deviation, Standardabweichung

SE

engl. standard error, Standardfehler

Tab.

Tabelle

TKG

Tausendkorngewicht

TrM

Trockenmasse

$\mathrm{X}_{\mathrm{m}}$

arithmetischer Mittelwert 


\section{Einleitung}

Die Winterrapsbestände weisen hinsichtlich ihrer Bestandesdichte und Einzelpflanzenentwicklung vielfach eine erhebliche Variabilität auf: In Abhängigkeit von Aussaatstärke, Feldaufgang und Auswinterung können die Pflanzendichten in einem weiten Bereich zwischen 20 und über 80 Pflanzen $/ \mathrm{m}^{2}$ schwanken (GEISLER \& STOY 1987). Insbesondere der gegenwärtig zunehmend an Bedeutung gewinnende Anbau von wuchsstarken MSL-Hybridsorten führt mit empfohlenen Saatstärken von nur 35 - $50 \mathrm{Körnern} / \mathrm{m}^{2}$ gegenüber den bei konventionellen OPSorten verwendeten 50 - 80 Körnern $/ \mathrm{m}^{2}$ (SAUERMANN \& GRONOW 2000) zukünftig zu erheblich geringeren Bestandesdichten. Verschiedene Pflanzendichten von Raps haben deutliche Unterschiede in der Einzelpflanzenentwicklung zur Folge: In Abhängigkeit von dem Standraum der Einzelpflanze variieren morphologische und phänologische Entwicklungsparameter der Rapspflanzen, wie z. B. Sprosstrockenmasse (HENNING 1979, Geisler \& HeNNING 1981a, SCARISBRICK et al. 1982), Wurzelmasse (MAKOWSKI 1995), Wurzelhalsdurchmesser (SCHULZ 1994, MaKowsKi 1995, Dosdall et al. 1996), Sprosslänge (HENNING 1979, SCARISBRicK et al. 1982, MAKOWSKI 1995), Blattlänge (CIPOLLINI \& BERGELSON 2001), Anzahl Blätter (GEISLER \& HeNNING 1981a) und Seitentriebe (SCHRIMPF 1954, Clarke \& Simpson 1978, HeNNING 1979, Stoy 1981, Geisler \& StOy 1987, МомOH \& ZHOU 2001) sowie BBCH-Stadium (HENNING 1979) erheblich. Je geringer Bestandesdichten gewählt werden und je mehr Standraum die Einzelpflanzen erhalten, desto kräftiger entwickelt sind sie.

Die Veränderung der Bestandesdichte und der Pflanzenarchitektur kann jedoch Konsequenzen für die Interaktionen zwischen den Schadinsekten und ihrer Wirtspflanze haben. Bei der Wahl ihres Nahrungs- und Eiablagehabitates reagieren verschiedene herbivore Schadinsekten sehr unterschiedlich auf die Dichte ihrer Wirtspflanzen. Während einige Schädlinge wie Pieris rapae, Delia brassicae und Diuraphis noxia in niedrigen Pflanzendichten den größten Einzelpflanzenbefall mit Eiern und Larven aufweisen, erreichen andere in mittleren (z. B. Phyllotreta striolata) oder hohen Bestandesdichten (z. B. Phyllotreta cruciferae) ihre größte Häufigkeit pro Pflanze (CROMARTIE 1975, Finch \& SKINNER 1976, BASKY \& HoPPER 2000). Die Ursachen für diese Präferenzen sind sehr vielfältig. Pflanzen in geringer Dichte können ausgewählt werden, weil sie größer oder infolge verminderter intraspezifischer Konkurrenz von besserer Qualität sind (BEGON et al. 1991, HonÉK et al. 1998, HoNÉK \& MARTINKOVA 1999, 2004). Dichtere Pflanzenbestände weisen z.T. einen stärkeren Herbivorenbefall auf als solche mit geringer Pflanzendichte (PIMENTEL 1961). Ausschlaggebend sind dabei das größere Nahrungsangebot und das günstigere Mikroklima dichter Pflanzenbestände sowie die räumliche 
Verteilung der Pflanzen, die das schnelle Wachstum und die Verbreitung der Schadinsekten erleichtern (RoOt 1973, Asjakin 1986, Reader 1992, Cipollini \& Bergelson 2001). Die intraspezifische Konkurrenz um Licht, Wasser und Nährstoffe reduziert möglicherweise in dichten Beständen darüber hinaus das Abwehrvermögen der Pflanzen gegenüber Herbivoren, da in ihnen die Synthese pflanzlicher Abwehrstoffe nur eingeschränkt erfolgen kann (CIPOLLINI \& Bergelson 2001). Die Pflanzendichte und das sich daraus ergebende Mikroklima im Bestand haben zudem Einfluss auf die Abundanz und Aktivität wichtiger natürlicher Gegenspieler wie Carabiden, Coccinelliden, Lycosiden und Syrphiden (HoNÉK 1979, 1983, 1985, 1988, HoNÉK \& JAROSÍK 2000, HASKEN 2001) und wirken so indirekt auch auf die Populationsentwicklung der Herbivoren.

Die sich mit der Bestandesdichte ändernde Pflanzenarchitektur kann auch direkt Einfluss auf die Herbivoren-Wirtspflanzen-Interaktionen nehmen. Einige Herbivoren wählen aufgrund der besseren Nahrungsqualität bevorzugt besonders kräftig entwickelte Wirtspflanzen (KOUBAITI \& Lerin 1992, Dosdall et al. 1996, CostA-Arbulú \& SÁNCHEZ 2000) und erhöhen so die Fitness ihrer Nachkommen (CARR et al. 1998). Auch Auswirkungen auf die Koinzidenz zwischen Wirtspflanzen und Herbivoren sind möglich. Nach BÜCHI (1996) belegt C. napi Rapspflanzen nur bis zu einer bestimmten Pflanzenhöhe mit Eiern. Sehr wüchsige Hybridrapssorten könnten diese kritische Höhe in niedrigen Aussaatdichten sehr viel schneller durchwachsen und so die Möglichkeit für die Eiablage vorzeitig beenden. Die Pflanzenmorphologie wirkt sich darüber hinaus auch auf Wirt-Parasitoid-Interaktionen aus (CLOYD \& SADOF 2000). Große Stängeldurchmesser verringern die Effektivität von Parasitoden, da sich ihre stängelminierenden Wirte vermehrt außer Reichweite des Legebohrers befinden (NITZSCHE 1998).

Die geringeren Pflanzendichten könnten zu einem höheren Befall der Einzelpflanzen führen, da sich die Schädlinge auf eine kleinere Zahl von Wirtspflanzen verteilen müssen. Es ist jedoch zu erwarten, dass das Kompensations- und Regenerationsvermögen kräftig entwickelter Rapspflanzen in geringen Bestandesdichten gegenüber der Schädigung wesentlich höher ist als das von schwachen Pflanzen und erstere einen höheren Schädlingsbefall ohne Ertragsverluste tolerieren können. Dies gilt in besonderem Maße für Hybridrapssorten (LAMB 1989). Daraus könnte sich ein wichtiger Ansatzpunkt für den integrierten Rapsanbau ergeben. Die Reduktion der Pflanzendichten könnte eine Anhebung der Bekämpfungsschwellenwerte für die Schadorganismen erlauben und so zu einer Minimierung von Insektizidanwendungen führen.

Im Mittelpunkt der vorliegenden Arbeit stehen drei wichtige Triebschädlinge des Winterrapses (Brassica napus L.): der Rapserdfloh Psylliodes chrysocephala [L.] (Coleoptera; Chrysomelidae), der Grosse Rapsstängelrüssler Ceutorhynchus napi Gyll. und der Gefleckte Kohl- 
triebrüssler C. pallidactylus (Mrsh.) (Coleoptera; Curculionidae). Alle drei Arten sind im Untersuchungsgebiet häufig auftretende Arten mit stängelminierenden Larven. Die Biologie und Schadwirkung dieser Triebschädlinge wurde in zahlreichen Arbeiten beschrieben (MEUCHE 1940, 1944, KAUFMANN 1941a, 1941b, KÖRTING 1942, GODAN 1947, 1948, 1950, GÜNTHART 1949, Dosse 1951, Fritzsche 1956, SchMUTTERER 1956, DMOCH 1958, 1959, BuHL 1959, Mohr 1960, Schulz 1983, 1985, BRoschewitz 1985, OPPERMANN 1990, FurLAN 1991, ALFORD et al. 2003).

Zum Einfluss unterschiedlicher Aussaatstärken und der daraus resultierenden Unterschiede in der Pflanzendichte und Einzelpflanzenarchitektur auf die Befallsdichte und Schadwirkung oben genannter Triebschädlinge liegen in der Literatur bisher nur wenige Hinweise vor. Die bislang vorliegenden Studien zum Einfluss der Pflanzendichte auf den Rapsbefall mit Triebschädlingen beschränken sich auf $P$. chrysocephala und C. pallidactylus. KAUFMANN (1941b) fand in einem Standweitenversuch eine Präferenz des Rapserdflohs für Parzellen mit einer höheren Rapsdichte innerhalb der Reihe. Nach BENEDEK (1984) und BROSCHEWITZ (1985) werden hohe Pflanzendichten von Winterraps stärker mit dem Gefleckten Kohltriebrüssler befallen, weil sie ihm mehr Ressourcen für die Eiablage bieten. Auch hohe Pflanzendichten von Blumenkohl fördern die Besiedlungsdichte von C. pallidactylus (FINCH \& SKINNER 1976). Zum Einfluss der Pflanzenarchitektur auf die Abundanz und Schadwirkung der oben genannten Triebschädlinge liegen nur bei den beiden Rüsselkäfer-Arten vereinzelte Untersuchungen vor. BRoschewitz (1985) stellte bei dem Gefleckten Kohltriebrüssler fest, dass kräftige Pflanzen von Sommerraps zur Eiablage bevorzugt werden. Nach GÜNTHART (1949) werden die größeren Kohlpflanzen stärker mit $C$. napi und C. pallidactylus besiedelt als die kleineren. JOURDHEUIL (1961) und BÜCHI (1996) zeigten eine begrenzende Wirkung der Pflanzenlänge auf die Eiablage des Grossen Rapsstängelrüsslers. Bei dem Gefleckten Kohltriebrüßler stellte PALOSZ (1980) eine deutliche inverse Beziehung zwischen dem Befallsgrad und dem mittleren Kornertrag/Schote in kleineren Rapspflanzen mit weniger als 4 Seitentrieben fest. Kräftige Rapspflanzen, die sich bei Pflanzendichten von 50 bis 80 Pflanzen $/ \mathrm{m}^{2}$ entwickeln, können nach Broschewitz \& DAebeler (1987) einen Befall von 14 bis 20 C. pallidactylus-Larven pro Pflanze ohne Ertragseinbußen tolerieren.

Es ist bisher noch weitgehend unbekannt, ob und in welchem Umfang unter den gegenwärtigen Anbaubedingungen von Winterraps eine Reduzierung der Befallsstärke und Schadwirkung wichtiger Rapsschädlinge durch verminderte Pflanzendichten und entsprechend veränderte 
Architektur und Phänologie wüchsiger und kräftiger Pflanzen sowie deren Kompensationsund Regenerationsvermögen möglich ist.

Das Ziel der nachfolgend beschriebenen Forschungsarbeit war es daher, unter den Bedingungen des natürlichen Befallsdruckes zu untersuchen, welchen Einfluss unterschiedliche Aussaatstärken von Winterraps und die daraus resultierenden Pflanzendichten und Einzelpflanzenarchitektur auf die Befallsdichte, das räumlich-zeitliche Verteilungsmuster in den Pflanzen und die Schadwirkung der drei oben genannten Triebschädlinge sowie den Ertrag haben. 


\section{Material und Methoden}

\subsection{Untersuchungsstandorte}

Die Freilanduntersuchungen wurden auf den Versuchsflächen des Institutes für Pflanzenpathologie und Pflanzenschutz der Universität Göttingen in den Anbaujahren 1999/00 und 2000/01 am Standort Weendelsbreite und im Jahr 2001/02 am Standort Otto-Hahn-Straße durchgeführt. Beide Versuchsstandorte befinden sich in Göttingen-Weende am Nordrand des Stadtgebietes (Abb. 1). Die durchschnittliche Höhe über NN beträgt $200 \mathrm{~m}$. Die Untersuchungsstandorte können dem subatlantischen Klimaraum zugerechnet werden. Im langjährigen Mittel fallen $634 \mathrm{~mm}$ Niederschlag, die Jahresdurchschnittstemperatur beträgt $8,6^{\circ} \mathrm{C}$.

Die Versuchsflächen am Standort Weendelsbreite und Otto-Hahn-Straße werden seit vielen Jahren vorrangig mit einer dreigliedrigen Fruchtfolge (Winterraps-Winterweizen-Wintergerste) bewirtschaftet. Die Kultur Winterraps nimmt bezogen auf die gesamte bewirtschaftete Fläche mit ca. $25 \%$ einen hohen Anteil an der Anbaufläche ein. Die Nähe der Versuchsschläge zu den Überwinterungshabitaten sowie der häufige Verzicht auf Insektizidbehandlungen im Frühjahr boten optimale Bedingungen für eine Massenvermehrung der drei untersuchten Triebschädlinge.

\subsubsection{Standort Weendelsbreite}

Bei der Versuchsfläche Weendelsbreite handelt es sich um eine Parabraunerde aus Löß der Zustandsstufe 3 mit einer Bodenwertzahl von 80 und einer Ackerzahl von 82. In der Vegetationsperiode 1999/00 lag die Versuchsfläche nördlich des Weendelsgrabens, der mit Bäumen, Büschen und Gras bewachsen ist (Abb. 1, s. Markierung). Die Fläche weist eine leichte Hangneigung in Richtung Süden auf und grenzt im Norden an einen mit Feldgehölzen bewachsenen Feldweg mit dahinterliegenden Ackerflächen, im Westen an weitere Ackerflächen. Im Nordosten und Osten schließen sich oberhalb der Fläche bewaldete Hangflächen an, die im wesentlichen mit Buchenwald bestückt sind. Im Versuch 2000/01 befand sich die Untersuchungsfläche südlich des Weendelsgrabens (Abb. 1, s. Markierung), sie ist im Westen, Osten und Süden von Ackerflächen umgeben. Nach Norden schließt sich der Weendelsgraben mit dahinterliegenden Ackerflächen und im Nord-Osten ein Gartengrundstück an. 




Abb. 1: Lage der Versuchsflächen Weendelsbreite und Otto-Hahn-Straße 


\subsubsection{Standort Otto-Hahn-Straße}

Bei der Versuchsfläche Otto-Hahn-Straße handelt es sich um einen Kalkstein-Verwitterungsboden der Zustandsstufe 3 mit einer Bodenwertzahl von 68 und einer Ackerzahl 68. Die Versuchsfläche lag ca. 300m östlich vom Standort Weendelsbreite und grenzt im Norden, Osten und Süden an heckenartige Baum- und Gebüschsäume, im Westen an eine bebaute Ackerfläche an (Abb. 1, s. Markierung).

\subsection{Witterungsverlauf 1999-2002}

Die Wetterdaten wurden während des gesamten Untersuchungszeitraumes von einer ca. 1,5 $\mathrm{km}$ von dem Versuchsstandort Weendelsbreite entfernt liegenden Wetterstation aufgezeichnet. Neben der durchschnittlichen, maximalen und minimalen Lufttemperatur (2 m Höhe), der Niederschlagsmenge, der Windgeschwindigkeit (2 m Höhe) und der relativen Luftfeuchte wurde auch die Bodentemperatur (10 cm Tiefe) sowie die Sonneneinstrahlung ( $3 \mathrm{~m}$ Höhe) erfasst.

Die Monatsmittelwerte der Temperatur und die Niederschlagsmenge im August 1999 entsprachen denen der langjährigen Mittelwerte der Jahre 1951-1980 (Abb. 2a). Der September fiel mit einer Monatsdurchschnittstemperatur von $17,4{ }^{\circ} \mathrm{C}$ ungewöhnlich warm aus. Auch die Niederschlagsmenge blieb $16 \mathrm{~mm}$ unter dem langjährigen Durchschnittswert. Die Monate Dezember 1999 bis Mai 2000 zeichneten sich ebenfalls durch zu hohe Temperaturen aus. Insbesondere der Februar war mit einem Durchschnittswert von $4,3{ }^{\circ} \mathrm{C}$ außergewöhnlich mild und warm. Der Juli hingegen war etwas zu kalt. Die relativ geringe Niederschlagsneigung im September und November setzte sich auch im April, Mai und Juni weiter fort. Überdurchschnittlich starke Niederschläge waren in den Monaten Dezember bis März sowie im Juli festzustellen.

Das Winterhalbjahr des Anbaujahres 2000/01 fiel durch überdurchschnittlich hohe Temperaturen auf (Abb. 2b). In den Monaten August bis Februar waren die Temperaturen um $0,9{ }^{\circ} \mathrm{C}$ bis 2,1 ${ }^{\circ} \mathrm{C}$ höher als die langjährigen Mittelwerte. Auch Mai und Juli waren ungewöhnlich warm. Relativ hohe Niederschläge fielen im September, März und Juli (51,7 mm, 42,5 mm bzw. 19,1 mm höher als im langjährigen Mittel). Die Monate Oktober und Mai waren hingegen zu trocken (15,5 mm bzw. 28,0 mm geringer als im langjährigen Mittel).

Insbesondere der September 2001 war außergewöhnlich feucht und kalt (61,1 mm über dem langjährigen Mittel bzw. 1,7 ${ }^{\circ} \mathrm{C}$ unter dem langjährigen Mittel) (Abb. 2c). Vergleichsweise hohe Niederschläge fielen auch im Februar und Mai (44,7 mm bzw. 58,7 mm höher als im 
langjährigen Mittel). Die Monate Dezember, Juni und Juli hingegen waren sehr trocken (24,3 mm, 43,6 mm bzw. 44,6 mm geringer als im langjährigen Mittel). Den im langjährigen Vergleich relativ kühlen Monaten September und Dezember stehen die wärmeren Monate August, Oktober, Januar, Februar und März gegenüber. Vor allem in den Monaten August, Oktober und Februar lag der Monatsmittelwert um $2,1^{\circ} \mathrm{C}, 3,3{ }^{\circ} \mathrm{C}$ bzw. $4,5^{\circ} \mathrm{C}$ höher als üblich.

Die drei Vegetationsperioden 1999/00, 2000/01 und 2001/02 erwiesen sich insgesamt mit Jahresdurchschnittswerten von $9,8{ }^{\circ} \mathrm{C}, 9,4{ }^{\circ} \mathrm{C}$ bzw. $9,4{ }^{\circ} \mathrm{C}$ und Jahressummen von $712,6 \mathrm{~mm}$, 679,4 mm bzw. 725,1 mm Niederschlag im Vergleich zu den langjährigen Mittelwerten von $8,6{ }^{\circ} \mathrm{C}$ bzw. $634 \mathrm{~mm}$ als zu mild und zu niederschlagsreich. 

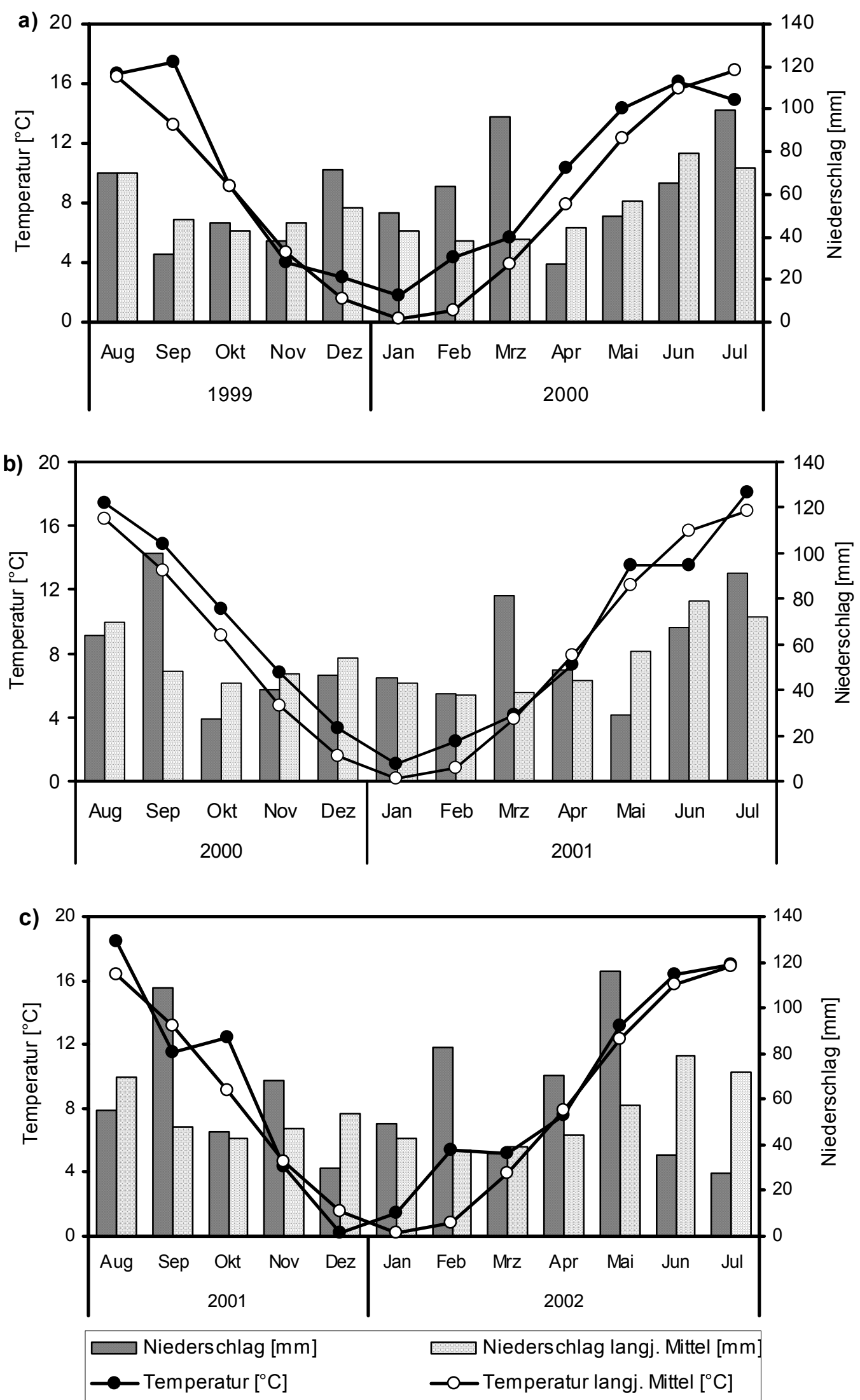

Abb. 2: Monatsmittelwerte der Lufttemperatur und des Niederschlags in den Versuchsjahren 1999 bis 2002 im Vergleich zum langjährigen Mittel (1951-1980) 


\subsection{Pflanzenbauliche Maßnahmen}

Der Winterraps wurde auf den Versuchsflächen Weendelsbreite und Otto-Hahn-Straße in allen Versuchsjahren praxisüblich bewirtschaftet. Auf den Einsatz von Insektiziden gegen C. napi, C. pallidactylus und andere Schädlinge im Frühjahr wurde jedoch verzichtet. Eine Insektizidbehandlung gegen $P$. chrysocephala wurde im Herbst der drei Jahre nach dem Überschreiten der Bekämpfungsschwelle (3 - 5 Larven/Pflanze, GARBE et al. 1996) vorgenommen. Im 1. Versuchsjahr wurden die ganzen Versuchsparzellen behandelt, im 2. und 3. Jahr jeweils nur eine Parzellenhälfte (Abb. 3a bis 3c, Kap. 2.5). Die entomologischen Untersuchungen im Rahmen der vorliegenden Arbeit wurden ausschließlich in den unbehandelten Teilflächen durchgeführt. In den Mantelsaaten wurde keine Insektizidbehandlung durchgeführt. Die Stickstoffdüngung erfolgte in betriebsüblicher Höhe unter Berücksichtigung der bei Vegetationsbeginn im Frühjahr im Boden gefundenen Menge an mineralischem Stickstoff. Die Details der einzelnen pflanzenbaulichen Maßnahmen sind Tabelle $1 \mathrm{zu}$ entnehmen.

Tab. 1: Pflanzenbauliche Maßnahmen in den Anbaujahren 1999/00 - 2001/02

\begin{tabular}{|c|c|c|c|}
\hline Anbaujahr & $1999 / 2000$ & $2000 / 2001$ & $2001 / 2002$ \\
\hline Vorfrucht & Winterroggen & Wintergerste & Winterweizen \\
\hline $\begin{array}{l}\text { Boden- } \\
\text { bearbeitung }\end{array}$ & $\begin{array}{l}\text { Pflug, } \\
2 \times \text { Kreiselegge }\end{array}$ & $\begin{array}{l}\text { Pflug, Walze, } \\
2 \times \text { Kreiselegge }\end{array}$ & $\begin{array}{l}\text { Pflug, } 2 \times \text { Walze, } \\
2 \times \text { Kreiselegge }\end{array}$ \\
\hline Aussaattermin & 23.08 .99 & 24.08 .00 & 28.08 .01 \\
\hline Saatstärke & $\begin{array}{l}2,1 \mathrm{~kg} / \mathrm{ha}=30 \mathrm{Körner} / \mathrm{m}^{2} \\
4,1 \mathrm{~kg} / \mathrm{ha}=60 \mathrm{Körner} / \mathrm{m}^{2} \\
6,2 \mathrm{~kg} / \mathrm{ha}=90 \mathrm{Körner} / \mathrm{m}^{2}\end{array}$ & $\begin{array}{l}2,1 \mathrm{~kg} / \mathrm{ha}=30 \mathrm{Körner} / \mathrm{m}^{2} \\
4,1 \mathrm{~kg} / \mathrm{ha}=60 \mathrm{Körner} / \mathrm{m}^{2} \\
6,2 \mathrm{~kg} / \mathrm{ha}=90 \mathrm{Körner} / \mathrm{m}^{2}\end{array}$ & $\begin{array}{l}2,1 \mathrm{~kg} / \mathrm{ha}=30 \mathrm{Körner} / \mathrm{m}^{2} \\
4,1 \mathrm{~kg} / \mathrm{ha}=60 \mathrm{Körner} / \mathrm{m}^{2}\end{array}$ \\
\hline Sorte & Mohican & Artus & Artus \\
\hline Saatgutbeizung & Oftanol T & $\begin{array}{l}\text { Gaucho WS } 70 \text { + Contur plus } \\
+ \text { TMTD + DMM }\end{array}$ & Chinook + TMTD + DMM \\
\hline $\begin{array}{l}\text { N-Gaben } \\
\text { (Düngerform) }\end{array}$ & $\begin{array}{lll}21.03 .00 & 80 \mathrm{~kg} & (\mathrm{AHL}) \\
03.04 .00 & 35 \mathrm{~kg} & (\mathrm{SSA}) \\
11.04 .00 & 80 \mathrm{~kg} & (\mathrm{AHL}) \\
\end{array}$ & $\begin{array}{lll}02.04 .01 & 80 \mathrm{~kg} & (\mathrm{AHL}) \\
04.04 .01 & 35 \mathrm{~kg} & (\mathrm{SSA}) \\
12.04 .01 & 80 \mathrm{~kg} & (\mathrm{AHL}) \\
\end{array}$ & $\begin{array}{lll}11.03 .02 & 80 \mathrm{~kg} & (\mathrm{AHL}) \\
18.03 .02 & 35 \mathrm{~kg} & (\mathrm{SSA}) \\
03.04 .02 & 80 \mathrm{~kg} & (\mathrm{AHL}) \\
\end{array}$ \\
\hline Herbizideinsatz & $\begin{array}{l}\text { 03.09.99 } \text { Butisan Top 2,0 I/ha } \\
\text { 19.10.99 Pradone Kombi 3,5 kg/ha }\end{array}$ & 25.08.00 Brasan 2,5 I/ha & 29.08.01 Brasan 2,5 I/ha \\
\hline Wachstumsregler & 11.04.00 CCC $7201 \mathrm{l} / \mathrm{ha}$ & 09.04.01 CCC $7201 \mathrm{l} / \mathrm{ha}$ & \\
\hline Fungizideinsatz & 11.04.00 Folicur 0,5 I/ha & 09.04.01 Folicur 0,5 I/ha & \\
\hline Insektizideinsatz & 29.11.99 Karate WG $100 \mathrm{~g} / \mathrm{ha}$ & 09.11.00 Karate WG $100 \mathrm{~g} / \mathrm{ha}$ & 13.12.01 Karate WG $100 \mathrm{~g} / \mathrm{ha}$ \\
\hline Schneckenkorn & 24.08.99 Metarex 3,5 kg/ha & 24.08.00 Metarex 3,5 kg/ha & $\begin{array}{l}\text { 28.08.01 Metarex } 6 \mathrm{~kg} / \mathrm{ha} \\
\text { 13.09.01 Metarex } 6 \mathrm{~kg} / \mathrm{ha} \\
\text { 19.10.01 Metarex } 6 \mathrm{~kg} / \mathrm{ha}\end{array}$ \\
\hline Erntetermin & 21.07 .00 & 25.07 .01 & 31.07 .02 \\
\hline
\end{tabular}

\subsection{Ermittlung der Pflanzendichte}

Die Pflanzendichte wurde in allen Versuchsjahren im Herbst im 3-4-Blattstadium und im Frühjahr kurz nach Beginn des Längenwachstums des Winterrapses mit Hilfe eines 0,25 $\mathrm{m}^{2}$ 
großen Zählrahmens aus Holz ermittelt (Tab. 2). Die Erhebungen erfolgten zufällig entlang eines Transektes. Im Anbaujahr 1999/00 wurden die Pflanzen im Herbst und Frühjahr an 8 Stellen pro Parzelle $(8 * 6=48$ Stellen/Saatvariante) gezählt. In den Jahren 2000/01 und 2001/02 wurden die Pflanzen in den unbehandelten und behandelten Teilflächen an je 10 Stellen pro Parzelle ( $10 * 6=60$ Stellen pro behandelte bzw. unbehandelte Saatvariante) gezählt. Nach einem sehr hohen Befall von $P$. chrysocephala im Herbst 2000 wurde die Anzahl der Pflanzen ohne Haupttrieb durch Untersuchung der Stoppeln mit dem oben genannten Holzzählrahmen nach der Ernte Ende Juli bestimmt. Die Zählung der Stoppeln wurde nur in den unbehandelten Parzellen an 14 Stellen pro Parzelle $(14 * 6=84$ Stellen/Variante) durchgeführt. Die mittleren Pflanzendichten pro $\mathrm{m}^{2}$ und die Pflanzenverluste über Winter konnten anschließend rechnerisch ermittelt werden.

Tab. 2: Termine der Zählungen von Rapspflanzen und -stoppeln zur Ermittlung der Bestandesdichte in den Versuchsjahren 1999 bis 2002

\begin{tabular}{cccc}
\hline Versuchsjahr & Herbst & Frühjahr & Sommer \\
\hline $1999 / 00$ & 28. Sep. & 22. Mrz. & - \\
$2000 / 01$ & 19. Sep. & 04. Apr. & 31. Jul. \\
$2001 / 02$ & 18. Okt. & 27. Mrz. & - \\
\hline
\end{tabular}

\subsection{Versuchsanlage}

Mit Ausnahme des Anbaujahres 2001/02 wurden die Versuche als randomisierte Blockanlage angelegt (Abb. 3a bis 3c). In den Anbaujahren 1999/00 und 2000/01 wurden drei Saatstärken von 30, 60 und $90 \mathrm{Körnern} / \mathrm{m}^{2}$, im Jahr 2001/02 zwei Saatstärken von 30 und $60 \mathrm{Körnern} / \mathrm{m}^{2}$ ausgesät. Die Parzellen mit gleicher Saatstärke wurden in allen Versuchen 6-fach wiederholt. Die Parzellengröße betrug im 1. Jahr 7,5 m x 15 m, im 2. Jahr 15 m x 20 m und im 3. Jahr 15 $\mathrm{m} \times 22,5 \mathrm{~m} . \mathrm{Im} 1$. und 2. Versuchsjahr wurde je ein 7,5 m breiter Mantelsaatstreifen zwischen den Parzellenblöcken und in allen Jahren ein mindestens $5 \mathrm{~m}$ breiter Mantelsaatstreifen um die Versuchsflächen herum angelegt. In allen Mantelsaaten wurden $60 \mathrm{Körner} / \mathrm{m}^{2}$ ausgesät. Die Größe der Versuchsfläche lag im Versuchsjahr 1999/00 bei ca. 0,3 ha (22,5 m x 127,5 m), im Jahr 2000/01 bei ca. 0,7 ha (45 m x 157,5 m) und im Jahr 2001/02 bei 0,6 ha (67,5 m x $90 \mathrm{~m})$. Im 1. Versuchsjahr wurde die Liniensorte Mohican, im 2. und 3. Jahr die Hybridsorte Artus angebaut. Die Aussaat erfolgte in allen Versuchsjahren mit einer Scheibenschardrillmaschine mit einem Reihenabstand von 13,2 cm. Der Aussaattermin fiel stets in die letzte Augustdekade. 
a) 1999/00 (Weendelsbreite)

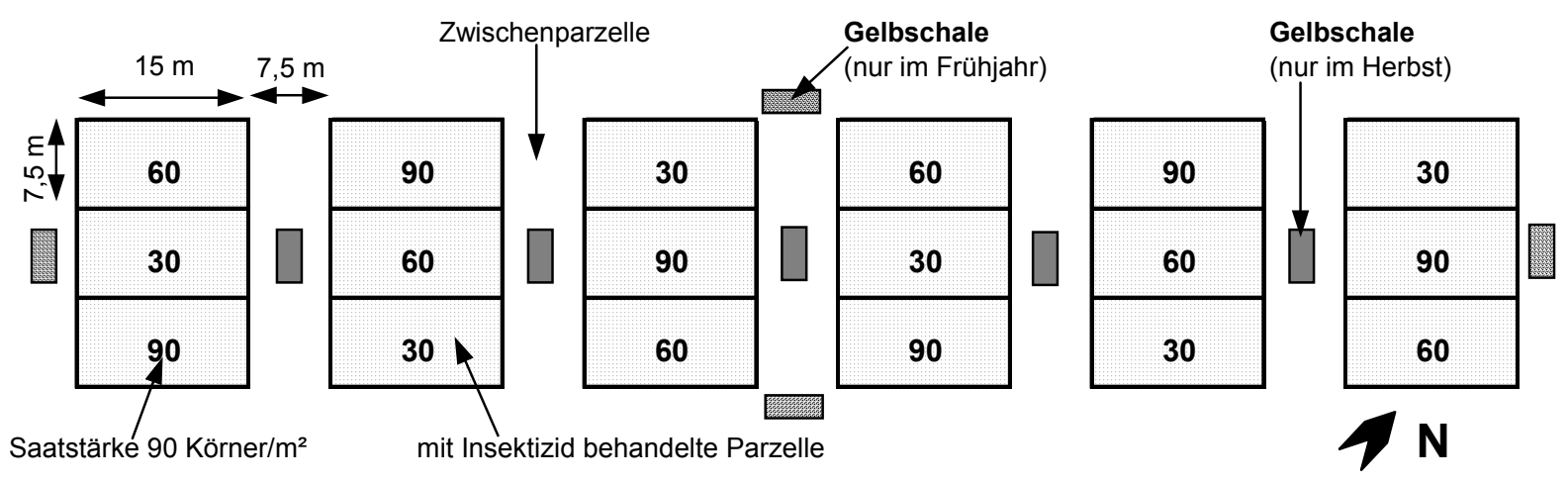

b) 2000/01 (Weendelsbreite)

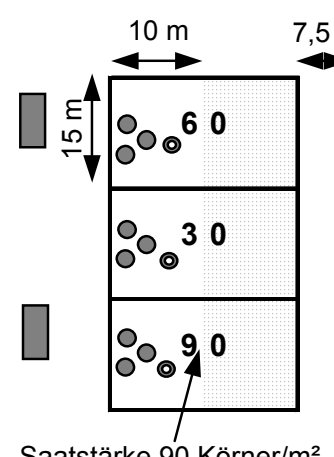

Saatstärke 90 Körner/m²

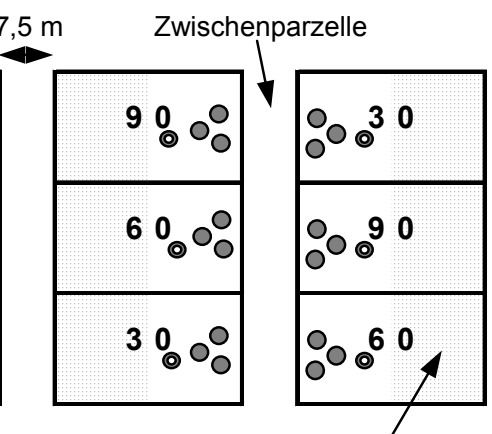

mit Insektizid behandelte Parzelle
Bodenphotoeklektor

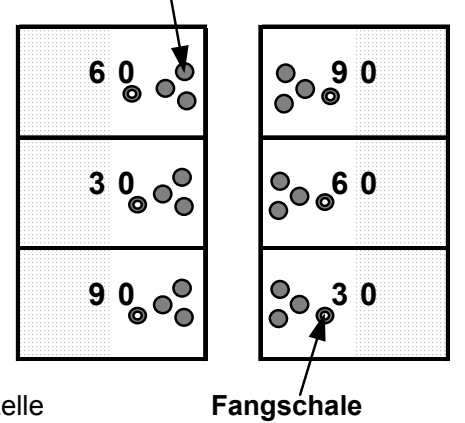

Gelbschale

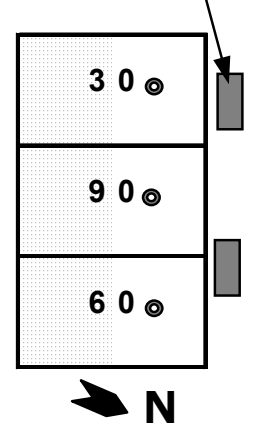

c) $2001 / 02$ (Otto-Hahn-Straße)

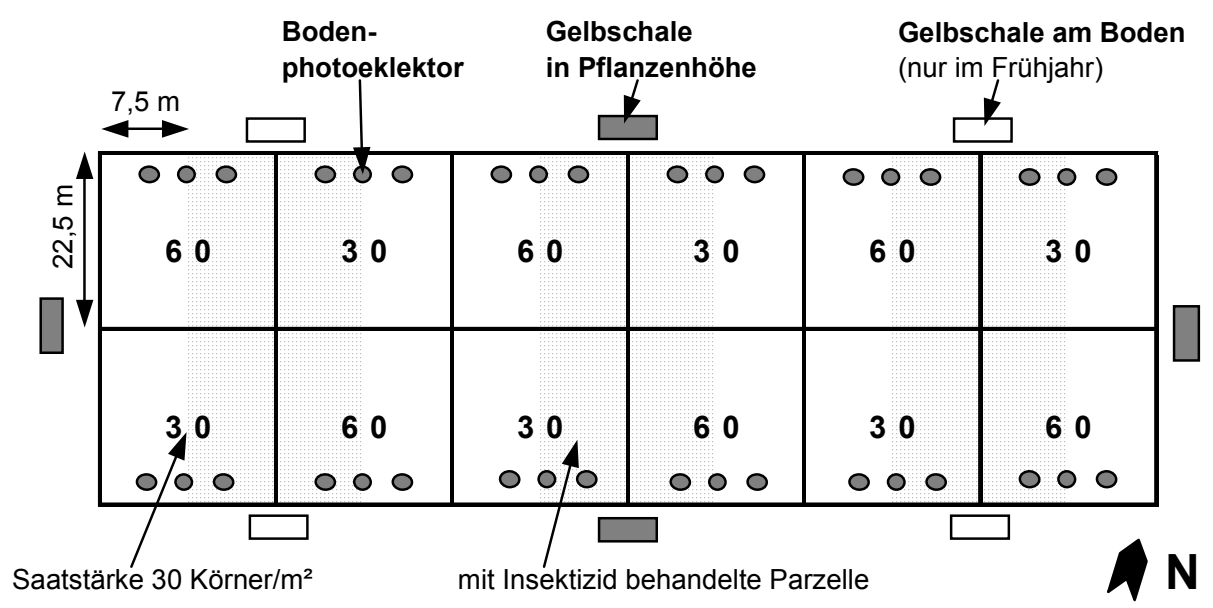

Abb. 3: Schematische Darstellung der Versuchsanlagen und der räumlichen Verteilung der Stichproben in den Versuchsjahren 1999/00, 2000/01 (Weendelsbreite) und 2001/02 (Otto-Hahn-Straße) 


\subsection{Auswertungsmethoden}

\subsubsection{Bestimmung der Triebschädlinge}

Die Determination von P. chrysocephala, C. napi und C. pallidactylus wurde unter einem lichtstarken Zeiss-Binokular mit 8- bis 100-facher Vergrößerung durchgeführt. Bei der Bestimmung der Imagines fanden Angaben von Schulz (1983), BROSCHEWITZ (1985) und OPPERMANN (1990) Verwendung. Die Imagines von P. chrysocephala sind 3 bis 4,5 mm große, glänzende, blauschwarze, länglich-ovale Blattkäfer. Die Imagines von C. pallidactylus sind 2,5 bis 3,5 mm lang. Durch eine unregelmäßig verteilte, weißgraue, schuppenartige Behaarung sehen die Käfer fleckig aus. Sie haben einen dünnen, abwärts gebogenen Rüssel, rötlichgelbe bis rostbraune Tarsen und einen hellen kreisförmigen Fleck an der Flügeldeckenbasis. Die Imagines von $C$. napi sind 3,2 bis $4 \mathrm{~mm}$ lange, im Grundton einheitlich schwarz gefärbte Rüsselkäfer, die durch eine schuppige Behaarung schiefergrau erscheinen. Die drei bis vier nebeneinander liegenden Schuppenreihen innerhalb der Zwischenräume der Flügeldecken unterscheiden C. napi von der ähnlich aussehenden Art C. assimilis, die lediglich eine bis drei unregelmäßige Schuppenreihen innerhalb deutlich engerer Zwischenräume aufweist (FREUDE et al. 1983).

Die Differenzierung der Geschlechter erfolgte anhand morphologischer Merkmale. Die Geschlechter von $P$. chrysocephala unterscheiden sich dadurch, dass die Männchen ein deutlich verbreitertes, herzförmiges erstes Tarsenglied besitzen, während es bei den Weibchen zylindrisch und von nahezu konstantem Durchmesser ist (KAUfMann 1941a, Schulz 1983). Die Männchen von C. napi und C. pallidactylus besitzen am Ende der Mittel- und Hinterschienen auf der Innenseite einen kräftigen Dornfortsatz, der winkelförmig nach innen geknickt ist. An den Extremitäten der Weibchen fehlt dieser Dorn. Ein im letzten Abdominalsternit der Männchen befindliches Grübchen, in dem bei der Kopulation das Pygidium des Weibchens liegt, stellt ein weiteres sekundäres Geschlechtsmerkmal dar (GÜNTHART 1949, BROSCHEWITZ 1985, OPPERMANN 1990).

Die Unterscheidung der Eier der beiden Ceutorhynchus-Arten in den Trieben lässt sich anhand ihrer Größe und Form vornehmen. Die Eier von C. napi sind mit einer Länge von 0,80 mm und einer Breite von 0,45 mm (OPPERMANN 1990) größer und schlanker als die Eier von $C$. pallidactylus (Länge: 0,56 mm, Breite: 0,38 mm, SPEYER 1921). Als weiteres Hilfsmittel bei der Bestimmung der Eier kann die Größe der Eigelege dienen. Die Weibchen von C. napi legen nur ein Ei pro Eigelege ab, während die Gelege von C. pallidactylus häufig 3 - 4 Eier enthalten (GÜNTHART 1949, BROSCHEWITZ 1985). Die Eier von P. chrysocephala werden in den 
Oberboden abgelegt. Sie sind oval, hellgelb gefärbt, trüb durchscheinend, und weisen außen eine Maschenstruktur auf, die ein polyedrisches Netz als Relief trägt. Sie haben eine Länge von 0,70 - 0,90 $\mathrm{mm}$ und eine Breite von 0,38 - 0,40 $\mathrm{mm}$ (Schulz 1983).

Im Gegensatz zu den beinlosen Larven von C. napi und C. pallidactylus zeichnen sich die Larven von P. chrysocephala durch das Vorhandensein von drei Beinpaaren aus. Die sichersten Unterscheidungsmerkmale der Larven der beiden Ceutorhynchus-Arten sind die Kutikulastruktur und die Beborstung der Kopfkapseln der Larven (GÜNTHART 1949, DOSSE 1953, SCHERF 1964, BrosCHEWITZ 1985, OpPERMANN 1990). Der gesamte Körper von C. napi ist mit warzenähnlichen Wülsten besetzt, die auf der Ventralseite klein und flach sind, dorsal dagegen stark gewölbt erscheinen. Die Larvenhaut von C. pallidactylus ist hingegen völlig glatt und glänzend. Die Kopfkapsel von C. napi weist am hinteren äußeren Rand eine Borste auf, die bei den Larven von C. pallidactylus fehlt. Als zusätzliches Hilfsmittel bei der Bestimmung der Larven kann die Farbe der Kopfkapseln dienen. Die Kopfkapsel von C. napi weist im 1. und 2. Larvenstadium eine schwärzlich-braune Färbung auf, während sie im 3. Larvenstadium und bei allen Larvenstadien von C. pallidactylus gelb ist (MEUCHE 1942).

Die drei Larvenstadien von P. chrysocephala (Schulz 1983), C. napi (Meuche 1942, GÜNTHART 1949, Dosse 1953) und C. pallidactylus (SPEYER 1921, GÜNTHART 1949, NiSSEN 1997) lassen sich anhand der Breite ihrer Kopfkapsel unterscheiden.

\subsubsection{Datenaufbereitung und Statistik}

Die Aufbereitung des Datenmaterials erfolgte mit den Programmen MS Excel (Version 2000), Xact (Version 7.02f2) und SigmaPlot (Version 2000). Die statistischen Datenanalysen wurden mit dem Statistikprogramm SYSTAT (Version 10) durchgeführt. Zur statistischen Absicherung der Untersuchungsergebnisse dienten die Mittelwerte der Messdaten je Wiederholungsparzelle und Termin. Signifikante Unterschiede $(\mathrm{p} \leq 0,05)$ sind im Ergebnisteil durch unterschiedliche Buchstaben gekennzeichnet.

\subsubsection{Deskriptive Statistik}

$\underline{\text { Sexualindex }}$

Der Sexualindex beschreibt den Anteil der Weibchen in der Population (SCHWERDTFEgER 1968). Der Index nimmt Werte zwischen 0 und 1 an. 


\subsubsection{Analytische Statistik}

Alle empirisch gewonnenen Daten wurden zunächst auf Normalverteilung überprüft. Hierzu wurden je Stichprobe mit der Prozedur, Descriptive Statistics/Basic Statistics' Mean- und SDWerte berechnet. Die berechneten Werte wurden dann mit dem nicht parametrischen KOLMOGOROV-SMIRNOW-Test (Nonparametric Tests/One sample KS) auf Normalverteilung getestet. Lag eine Normalverteilung vor, wurde entweder eine einfaktorielle Varianzanalyse (ANOVA) mit anschließenden LSD- oder Tukey-Tests (bei unabhängigen 2 bzw. 3 Stichproben) durchgeführt oder ein t-Test (t-test/Two Groups) (bei verbundenen Stichproben) gerechnet. Die phänologischen Daten wurden mittels einer einfaktoriellen Varianzanalyse im ,repeated measurement design' (RM-ANOVA) analysiert. Wurden bei den Versuchen zwei Faktoren variiert, erfolgte die Berechnung einer zweifaktoriellen Varianzanalyse (ANOVA/Covariate(s)/Tukey). Die nicht normal verteilten Datensätze wurden dem nicht parametrischen KoLMOGOROV-SMIRNOW-Test unterzogen. Wurden die Versuchsvarianten weniger als sechsmal wiederholt, erfolgte der Vergleich zweier unabhängiger Stichproben durch die Anwendung des MANN-Whitney U-Tests (Nonparametric Tests/Kruskal-Wallis). Mehr als zwei unabhängige Stichproben wurden einer statistischen Auswertung mit Hilfe des KRUSKAL-WALLIS-Tests (Nonparametric Tests/ Kruskal-Wallis One-Way Analysis of Variance) unterzogen. Stellte sich bei dieser Berechnung signifikanter Unterschied heraus, schloss sich ein paarweiser Stichprobenvergleich mit dem Mann-Whitney U-Test und einer Präzisierung des Signifikanzniveaus nach DUNN-SZIDAK (SOKAL \& ROHLF 1995) an.

\section{$\underline{\text { ARCSINUS-Transformation }}$}

Prozentwerte wurden vor der statistischen Analyse zunächst in relative Zahlen umgewandelt und dann mit Hilfe des Statistikprogramms SigmaPlot (Version 2000) einer ARCSINUSTransformation unterzogen.

\section{Korrelationen und Regressionen}

Zur Ermittlung linearer Zusammenhänge zwischen zwei gleichwertigen Parametern wurden Korrelations- und Regressionsanalysen mit dem Programm Xact (Version 7.02f2) berechnet.

\subsection{Untersuchungsmethoden}

In den Freilanduntersuchungen an den Standorten Weendelsbreite und Otto-Hahn-Straße wurden zur Erfassung der Insekten Gelb- und Fangschalen, Bodenphotoeklektoren sowie Boden- 
und Pflanzenproben verwendet. Mittels Gelbschalen wurde zum einen der Flugbeginn von $C$. napi auf den Überwinterungsfeldern ermittelt, zum anderen dienten sie zur Kontrolle der $\mathrm{Zu}$ wanderung der Triebschädlinge in die Winterrapsfelder. Mit den Fangschalen wurde die Aktivitätsdichte, mit den Photoeklektoren die Abundanz der Imagines und mit den Bodenproben die Eidichte von $P$. chrysocephala innerhalb der Versuchsparzellen bestimmt. In den Pflanzenproben wurde die Abundanz und räumlich-zeitliche Verteilung der Larven von P. chrysocephala und der Eier und Larven von C. napi und C. pallidactylus sowie die Ausbildung der Einzelpflanzenparameter erfasst; zusätzlich wurden verschiedene Einzelpflanzenparameter ermittelt.

\subsubsection{Gelbschalen}

Der Aktivitätsbeginn von C. napi auf den Überwinterungsfeldern wurde mit Hilfe praxisüblicher Gelbschalen (Typ Zeneca, Maße 33 x 25 x 7,3 cm, ohne Abdeckgitter, Abb. 4) registriert. Jeweils eine Schale wurde in 5 bis $10 \mathrm{~m}$ Abstand vom Feldrand an jeder Seite des Feldes am Boden aufgestellt. Die Fallen wurden etwa zur Hälfte mit Wasser gefüllt und mit einigen Tropfen Entspannungsmittel (Pricol, 0,5 g/l) versetzt, damit die gefangenen Schadinsekten schneller absinken. Eine kleine Öffnung ca. $2 \mathrm{~cm}$ unterhalb der Schalenoberkante diente dazu, den Überlauf der Fangflüssigkeit bei Regen zu verhindern. Um die Attraktivität der Gelbschalen für die Schadinsekten zu erhalten, wurden die Schalen nach jeder Leerung mit einem großen Pinsel gereinigt und mit frischem Wasser aufgefüllt. Nach dem Leeren der Fallen wurden die Tiere zur Konservierung in 70 \%igen Alkohol überführt. Die Gelbschalen wurden täglich geleert. Die Fangzeiträume der Gelbschalen auf den Überwinterungsfeldern sind in Tabelle 3 wiedergegeben.

Auch auf den mit Raps bestellten Versuchsfeldern wurden Gelbschalen vom gleichen Typ installiert, um die Zuwanderung von $P$. chrysocephala im Herbst sowie von C. napi und $C$. pallidactylus im Frühjahr zu erfassen.

Die im Herbst eingesetzten Gelbschalen wurden auf den Boden aufgestellt. Im Herbst 1999 kamen 5 Schalen zum Einsatz, die in der Mitte der 5 Zwischenparzellen positioniert wurden (Abb. 3a). Im Herbst 2000 wurden jeweils 2 Schalen (insgesamt 4) im Abstand von 20 m zum Rand der Versuchsparzellen an den Kopfseiten des Feldes installiert (Abb. 3b). Im Herbst 2001 wurde jeweils eine Schale (insgesamt 4) im Abstand von 1 m zu den Kopf- und Längsseiten des Versuchsfeldes platziert (Abb. 3c). Die Fallenleerungen erfolgten zumeist in 7- 
tägigem Rhythmus, andernfalls wurden die Individuenzahlen auf eine 7-tägige Periode normiert.

Tab. 3: Fangzeiträume der Gelbschalen an den Standorten Weendelsbreite und Otto-HahnStraße in den Versuchsjahren 1999 bis 2002

\begin{tabular}{cccc}
\hline Jahr & Standort & Feld * & Fangzeitraum \\
\hline 1999 & Weendelsbreite & WR (REF) & 09.09.-04.11. \\
2000 & Weendelsbreite & ÜF (RSR) & $13.03 .-07.04$. \\
2000 & Weendelsbreite & WR (RSR, KTR) & 13.03.-07.04. \\
\hline 2000 & Weendelsbreite & WR (REF) & 06.09.-01.11. \\
2001 & Weendelsbreite & ÜF (RSR) & 28.03.-08.04. \\
2001 & Weendelsbreite & WR (RSR, KTR) & 28.03.-08.04. \\
\hline 2001 & Otto-Hahn-Straße & WR (REF) & $10.09 .-05.11$. \\
2002 & Weendelsbreite & ÜF (RSR) & $02.02 .-07.04$. \\
2002 & Otto-Hahn-Straße & WR (RSR, KTR) & $02.02 .-07.04$. \\
2002 & Otto-Hahn-Straße & WR ** (RSR, KTR) & $05.03 .-07.04$. \\
\hline
\end{tabular}

*: ÜF $=$ Überwinterungsfeld, WR $=$ Winterraps, $W_{R}^{* *}=$ Gelbschalen am Boden, REF $=$ Rapserdfloh, RSR $=$ Grosser Rapsstängelrüssler, KTR = Gefleckter Kohltriebrüssler
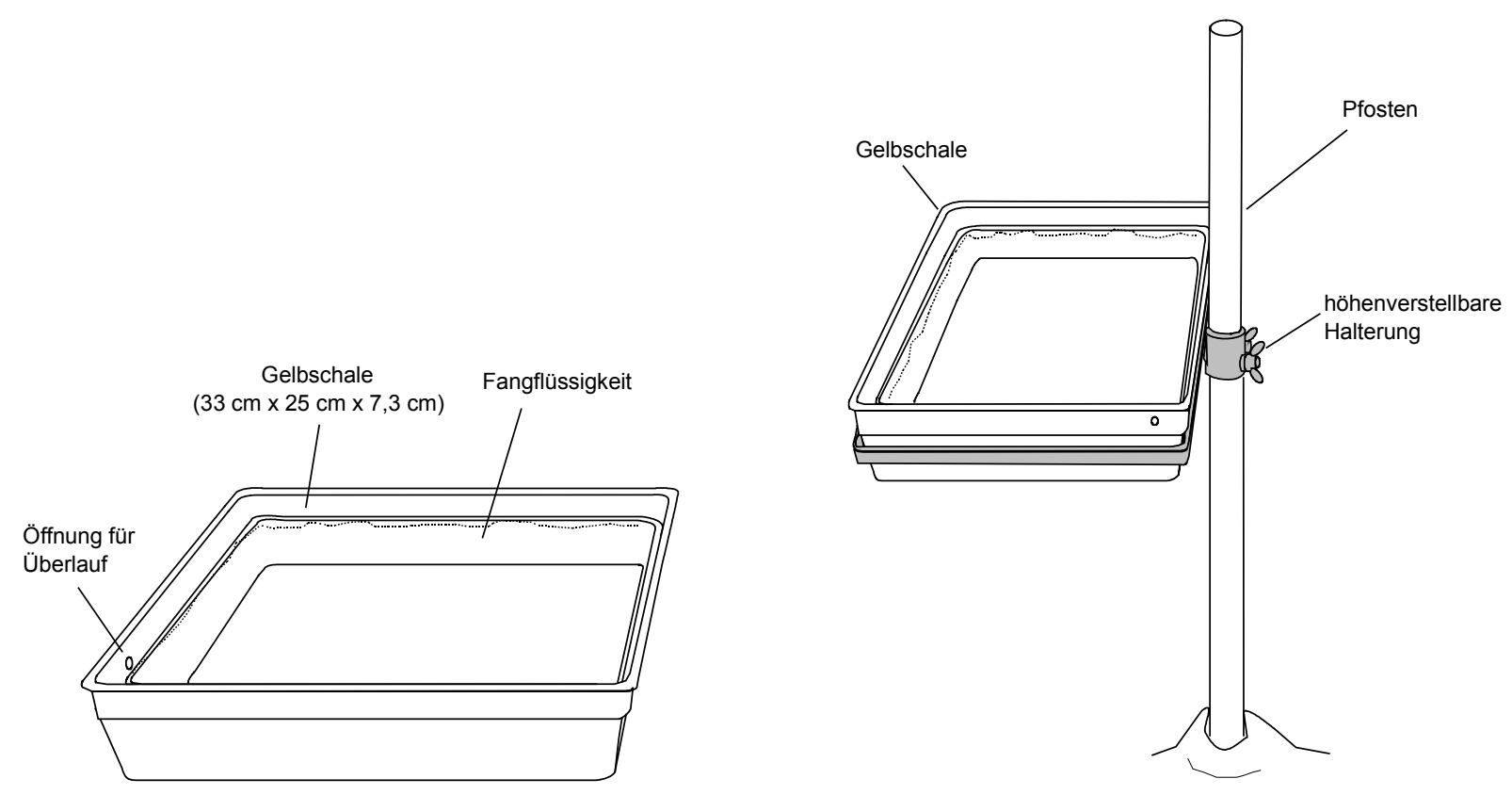

Abb. 4: Gelbschale (Typ Zeneca)

Im Frühjahr wurden die Gelbschalen zunächst am Boden belassen und zu Beginn des Längenwachstums an etwa $2 \mathrm{~m}$ lange Holzpfosten mit einer höhenverstellbaren Halterung befestigt (Abb. 4). Hierbei wurden die Schalen der jeweiligen Höhe der Pflanzen in der Weise an- 
gepasst, dass sie jeweils eine Handbreit über der Vegetation fixiert wurden. Die Gelbschalen standen im Frühjahr 2000 nicht mehr in den Zwischenparzellen, sondern jeweils eine Schale (insgesamt 4) wurde im Abstand von $1 \mathrm{~m}$ zu den Kopf- und Längsseiten des Versuchsfeldes positioniert (Abb. 3a). Die Fallenleerungen erfolgten in eintägigen Intervallen. Nach dem Aussortieren wurden die Tiere zur Konservierung in 70 \%igen Alkohol überführt.

Im Frühjahr 2002 kamen zusätzlich zu den 4 Gelbschalen, die der Pflanzenhöhe angepasst wurden, 4 weitere Gelbschalen (05.03.-07.04.) zum Einsatz, die auf der Bodenoberfläche aufgestellt waren. Sie sollten zur Klärung des Einwanderungsverhaltens (fliegende oder laufende Fortbewegung) dienen. Die Fangzeiträume der Gelbschalen auf den Winterrapsfeldern sind in Tabelle 3 wiedergegeben.

\subsubsection{Fangschalen}

Die Aktivitätsdichte von $P$. chrysocephala innerhalb der Versuchsparzellen wurde im Herbst 2000 mit Hilfe von Fangschalen erhoben. Da P. chrysocephala nicht durch farbige Schalen angelockt wird (SCHRÖDTER \& NOLTE 1954), konnten gelbe Plastikschalen (Ø $23 \mathrm{~cm}, 415,5$ $\mathrm{cm}^{2}$ ) verwendet werden. Jeweils eine Fangschale wurde in der Mitte der Versuchsparzelle am Boden aufgestellt. Die Fallen wurden etwa zur Hälfte mit Wasser gefüllt, das mit einem Entspannungsmittel (Pricol, 0,5 g/l) versetzt war. Die Schalen wurden nach jeder Leerung mit einem Pinsel gereinigt und mit frischem Wasser aufgefüllt. Die Leerung der Schalen wurde in 7-tätigen Intervallen vorgenommen. Zur Konservierung wurden die Tiere in 70 \%igen Alkohol überführt. Der Fangzeitraum im Herbst 2000 ist in Tabelle 4 wiedergegeben.

Tab. 4: Fangzeitraum der Fangschalen am Standort Weendelsbreite im Versuch 2000

\begin{tabular}{ccc}
\hline Jahr & Standort & Fangzeitraum \\
\hline 2000 & Weendelsbreite & 06.09.-02.11. \\
\hline
\end{tabular}

\subsubsection{Bodenphotoeklektoren}

Die Abundanz von P. chrysocephala im Rapsbestand wurde mit Bodenphotoeklektoren (Hersteller: Fa. Ecotech, Bonn, Abb. 5) ermittelt. Sie besaßen eine kreisförmige Grundfläche von $0,25 \mathrm{~m}^{2}$ und bestanden aus einem Kunststoffrahmen und einem schwarzen Stoffzelt mit Reißoder Klettverschluss, an dessen Spitze die Eklektorkopfdose (durchsichtiger Deckel, längerer Außenzylinder, leicht konischer Innenzylinder und Dosenstutzen) angebracht war. Das Prinzip 
dieser Fallenart beruht auf der positiv phototaktischen Orientierung der Tiere. Diese wandern zum Licht hin und gelangen dabei in die durchsichtige Eklektorkopfdose, die mit Ethylenglykol als Fangflüssigkeit gefüllt war. Um das Entweichen von Tieren in Bodennähe zu verhindern, wurden die Rahmen ca. 15 bis $20 \mathrm{~cm}$ tief in den Boden eingelassen und von außen etwa $20 \mathrm{~cm}$ hoch mit Boden angeschüttet. Die Kopfdosendeckel wurden mittels Gummis an der Kopfdose fixiert. Die Photoeklektoren wurden im Herbst der Jahre 2000 und 2001 mit je 3 Wiederholungen pro Parzelle (18 Eklektoren pro Saatstärke) in $3 \mathrm{~m}$ Abstand vom Parzellenrand entlang der Parzellenlängsseiten aufgebaut. Die Installation erfolgte in beiden Jahren eine Woche nach dem Erstauftreten der Rapserdflöhe in den Gelbschalen. Die Leerung der Eklektorkopfdosen wurde in 7-tätigen Intervallen vorgenommen. Der Durchlass zwischen Zelt und Kopfdose wurde regelmäßig auf Netze von Spinnen kontrolliert und bei Bedarf gereinigt, um die Fängigkeit der Photoeklektoren zu gewährleisten. Im Anschluss an die Kopfdosenleerung wurden die Eklektoren um ca. 2 m versetzt. Die Kopfdosenfänge wurden zur Konservierung in 70 \%igen Alkohol überführt. Die Fangzeiträume sind in Tabelle 5 wiedergegeben.
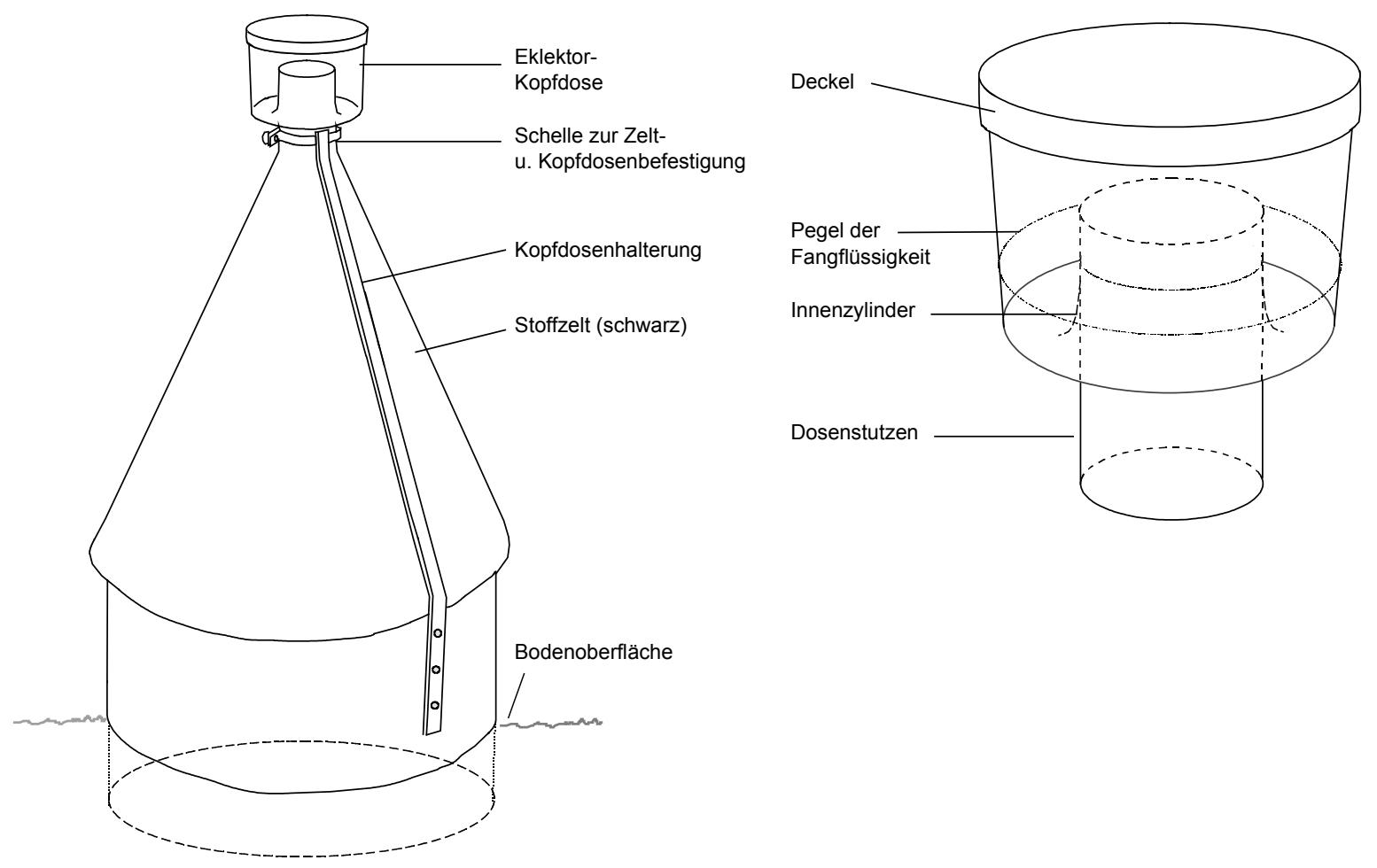

Abb. 5: Bodenphotoeklektor (verändert nach MÜHLENBERG 1993) 
Tab. 5: Fangzeiträume der Bodenphotoeklektoren an den Standorten Weendelsbreite und Otto-Hahn-Straße im Herbst der Jahre 2000 und 2001

\begin{tabular}{ccc}
\hline Versuchsjahr & Standort & Fangzeitraum \\
\hline 2000 & Weendelsbreite & 19.09.-02.10. \\
2001 & Otto-Hahn-Straße & $30.09 .-17.10$. \\
\hline
\end{tabular}

\subsubsection{Bodenproben}

Zur Ermittlung der Eidichten von $P$. chrysocephala in Abhängigkeit von unterschiedlichen Saatstärken wurden im Herbst der Jahre 2000 und 2001 an jeweils einem Termin Bodenproben entnommen. Im Herbst 2000 wurden die Bodenproben in allen drei Saatstärken unter Einschluss des Wurzelbereichs der Pflanzen und in der Saatstärke $30 \mathrm{Kö.} / \mathrm{m}^{2}$ auch außerhalb des Wurzelbereiches an pflanzenfreien Stellen genommen. Hierbei wurde eine rechteckige Stechform aus Kunststoff mit einer Grundfläche von $255 \mathrm{~cm}^{2}$ (Maße: 25,5 x 10 x $4 \mathrm{~cm}$ ) verwendet. Die oberen $4 \mathrm{~cm}$ des Bodens wurden abgetragen.

Im Herbst 2001 wurde die Methode in der Weise optimiert, dass die Bodenproben in allen Saatstärken in genau definierten Entfernungsstufen $(0-5 \mathrm{~cm}, 5-10 \mathrm{~cm}, 10-15 \mathrm{~cm})$ von den beprobten Pflanzen entnommen wurden. Die Probenahmen erfolgten mit einer rechteckigen Stechform aus Kunststoff mit einer Grundfläche von $45 \mathrm{~cm}^{2}$ (Maße: 9 x 5 x $4 \mathrm{~cm}$ ). Jede Probe setzte sich aus zwei Einstichen zusammen (Abb. 6). Im Herbst 2000 betrug der Stichprobenumfang je Saatstärkenvariante 15 Bodenproben (je 5 Proben in 3 Wiederholungsparzellen), im Herbst 2001 je Variante und Entfernungsstufe 24 Proben (je 6 Proben in 4 Wiederholungsparzellen).

Zusätzlich wurden die Gesamteidichten im Herbst 2001 in größeren Proben bestimmt. Hierzu wurde jeweils eine Fläche von $625 \mathrm{~cm}^{2}$ mit einem Holzrahmen abgesteckt, mit einem Spaten senkrecht zur Bodenoberfläche ausgestochen und bis zu einer Tiefe von $4 \mathrm{~cm}$ flach abgetragen. Bei den Saatstärken 30 und $60 \mathrm{Kö.} / \mathrm{m}^{2}$ wurden gezielt die Stellen ausgewählt, die im mittleren Bereich 2 bzw. 4 Pflanzen pro $1 / 16 \mathrm{~m}^{2}$ aufwiesen. Diese Pflanzendichte entsprach also etwa der Pflanzenzahl auf $1 / 16 \mathrm{~m}^{2}$ in der jeweiligen Saatstärke. Der Stichprobenumfang je Saatstärke lag bei 30 Bodenproben (je 5 Proben in 6 Wiederholungsparzellen). Alle Proben wurden einzeln in Plastikschalen mit einem Deckel verschlossen und im Kühlraum bei $2{ }^{\circ} \mathrm{C}$ gelagert, um die Eimortalität durch Bodenprädatoren in den Bodenproben auszuschließen.

Im Labor wurden die Eier mit gesättigter NaCl-Lösung (300 g/l) aus den Bodenproben aufgeschwemmt. Hierzu wurden die Proben in der Salzlösung vorsichtig zerkrümelt, kurz geschüt- 
telt und etwa 1 Stunde stehen gelassen. Anschließend wurde der wässrige Überstand und darin befindliche feine Wurzeln, Bodenpartikel, abgestorbene pflanzliche und tierische Substanz und Erdfloheier durch ein Metallsieb geseiht (Maschengröße $<0,38 \mathrm{~mm}$ ), mit Leitungswasser nachgespült und das aufgeschwemmte Material in 80 \%igen Alkohol überführt. Das Nachspülen mit Leitungswasser erfolgte durch mehrmaliges schnelle Eintauchen des Siebes in einen Wasserwirbel. Der Wasserwirbel wurde hierfür in einem flachen Spülbehälter durch einen starken Wasserstrahl erzeugt. Durch das schnelle Eintauchen und Herausnehmen des Siebes konnte die Verschlämmung der Siebmaschen vermieden werden. Vor dem Auszählen der Eier unter einem Binokular wurden die Proben zum Aufschwemmen der Eier erneut mit NaClLösung aufgefüllt und bis zum Absetzen von Schwebstoffen stehen gelassen.

Der günstigste Zeitpunkt der Probenahmen wurde durch stichprobenartige, wiederholte Sektion der Ovarien von Erdflohweibchen bestimmt (Tab. 6).

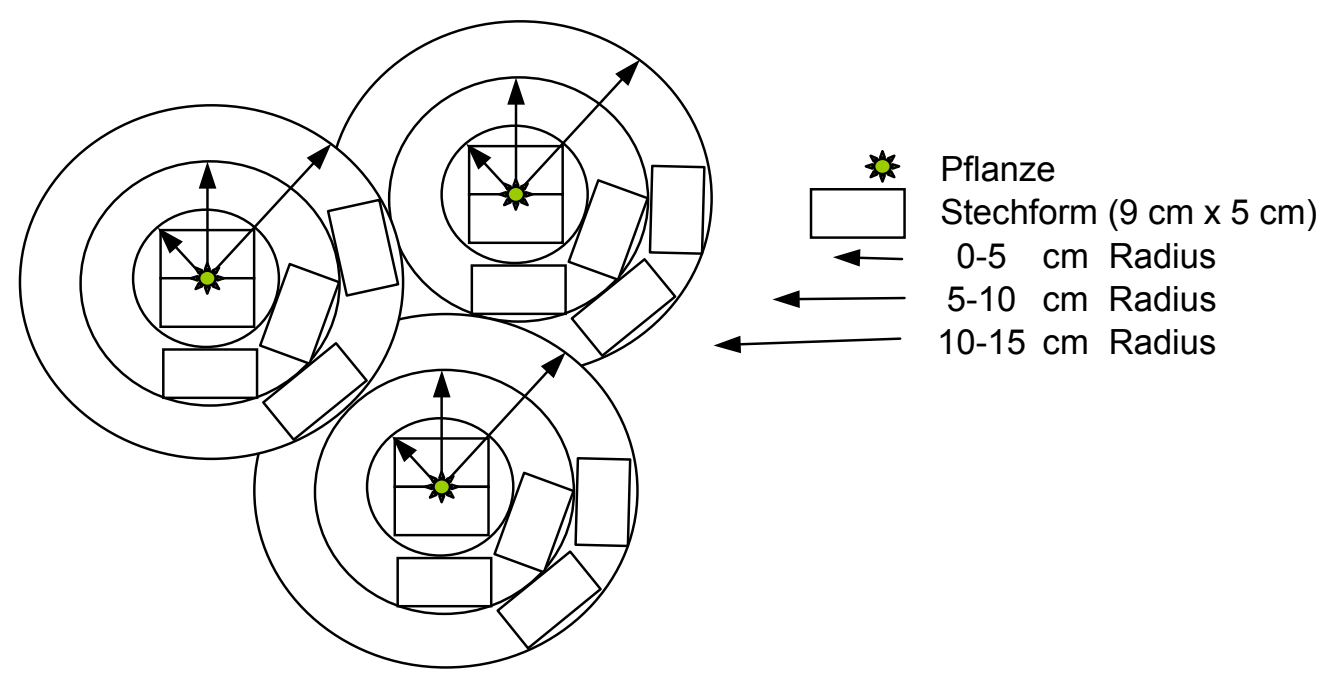

Abb. 6: Schematische Darstellung der Entnahme von Bodenproben in drei unterschiedlichen Entfernungsradien um die Rapspflanzen im Herbst 2001 am Standort Otto-HahnStraße

Tab. 6: Termine der Entnahme von Bodenproben und Art der Probenahme an den Standorten Weendelsbreite und Otto-Hahn-Straße im Herbst der Jahre 2000 und 2001

\begin{tabular}{cccc}
\hline Versuchsjahr & Standort & Art der Probenahme & Termin \\
\hline 2000 & Weendelsbreite & $\begin{array}{c}\text { innerhalb und außerhalb } \\
\text { des Wurzelbereiches }\end{array}$ & 14.10. \\
2001 & Otto-Hahn-Straße & flächenbezogen & 30.10. \\
2001 & Otto-Hahn-Straße & 3 Entfernungsstufen & 04.11. \\
\hline
\end{tabular}




\subsubsection{Pflanzenproben}

Zur Ermittlung der Abundanz und räumlich-zeitlichen Verteilung der Larven von P. chrysocephala und der Eigelege und Larven von C. napi und C. pallidactylus in den Pflanzen sowie zur Erfassung der Einzelpflanzenparameter wurden Rapspflanzen am Wurzelhals abgeschnitten und im Labor untersucht. Die Probenahmen erfolgten zufällig entlang eines Transektes an mehreren Terminen.

Um die Verteilung der Eigelege und Larven in den Pflanzen räumlich genauer zuordnen zu können, wurden die Blätter der verschiedenen Blattetagen, die Seitentriebe und der Haupttrieb getrennt untersucht. Die in den Blättern und Trieben befindlichen Eier und Larven wurden herauspräpariert und bis zu der Art und dem Entwicklungsstadium (Larven) bestimmt. Zuvor wurden die Sprosslänge, der Sprossdurchmesser am Wurzelhals und in $50 \mathrm{~cm}$ Höhe sowie die Anzahl der Blätter und Seitentriebe ermittelt. Zudem wurde im April 2000 und 2001 eine Einteilung der Pflanzen in Klassen mit unterschiedlichen Seitentrieblängen (8 Klassen, Tab. 7) vorgenommen. Im April 2001 wurde zusätzlich die Länge und der Durchmesser der Seitentriebe in $20 \mathrm{~cm}$ und $40 \mathrm{~cm}$ Sprosshöhe ermittelt. Im April 2002 wurden diese Parameter in drei definierten Höhenabschnitten der Hauptsprossachse $(0-20 \mathrm{~cm}, 20-40 \mathrm{~cm}$ und über $40 \mathrm{~cm})$ erfasst. Die Anzahl der Seitentriebe ( $>1 \mathrm{~cm}$ ) wurde jeweils im April und Mai der drei Versuchsjahre bestimmt. Im Mai 2002 wurde außerdem die Anzahl schotentragender und verkümmerter Seitentriebe registriert. Die einzelnen Termine zur Ermittlung der Pflanzenparameter sind in Tabelle 8 dargestellt.

Tab. 7: Klassifizierung der Seitentrieblänge im mittleren und oberen Drittel des Haupttriebes der Rapspflanzen

\begin{tabular}{ccc}
\hline Klasse & $\begin{array}{c}\text { Länge der Seitentriebe } \\
\text { im mittleren Drittel } \\
\text { des Haupttriebes }\end{array}$ & $\begin{array}{c}\text { Länge der Seitentriebe } \\
\text { im oberen Drittel } \\
\text { des Haupttriebes }\end{array}$ \\
\hline 1 & $<0,5 \mathrm{~cm}$ & $<0,5 \mathrm{~cm}$ \\
2 & $<1 \mathrm{~cm}$ & $<0,5 \mathrm{~cm}$ \\
3 & $1-4,9 \mathrm{~cm}$ & $<1 \mathrm{~cm}$ \\
4 & $1-4,9 \mathrm{~cm}$ & $1-4,9 \mathrm{~cm}$ \\
5 & $5-7,9 \mathrm{~cm}$ & $<5 \mathrm{~cm}$ \\
6 & $5-7,9 \mathrm{~cm}$ & $5-7,9 \mathrm{~cm}$ \\
7 & $>8 \mathrm{~cm}$ & $<8 \mathrm{~cm}$ \\
8 & $>8 \mathrm{~cm}$ & $>8 \mathrm{~cm}$ \\
\hline
\end{tabular}


Tab. 8: Probenahmetermine für die Ermittlung der Pflanzenwachstumsparameter an den Standorten Weendelsbreite und Otto-Hahn-Straße in den Jahren 1999 bis 2002

\begin{tabular}{cll}
\hline Versuchsjahr & Standort & Termin \\
\hline 1999 & Weendelsbreite & 11.11. \\
2000 & Weendelsbreite & 20.04. \\
2000 & Weendelsbreite & 12.05. \\
\hline 2000 & Weendelsbreite & 16.11. \\
2001 & Weendelsbreite & 24.04. \\
2001 & Weendelsbreite & 25.05. \\
\hline 2001 & Otto-Hahn-Straße & 29.11. \\
2002 & Otto-Hahn-Straße & 17.04. \\
2002 & Otto-Hahn-Straße & 21.05. \\
\hline
\end{tabular}

Die Befallsdichte und -verteilung der Larven von $P$. chrysocephala wurde im Herbst und Winter der drei Versuchsjahre erhoben. Zur Erfassung der räumlich-zeitlichen Dynamik des Larvenbefalls von $P$. chrysocephala in den Rapspflanzen wurden die Pflanzenproben in den Versuchen 2000/01 und 2001/02 im Gegensatz zum Versuch 1999/00 an 4 bzw. 3 Terminen gesammelt. Eine Aufstellung der Probetermine mit den entsprechenden Untersuchungszielen ist Tabelle $9 \mathrm{zu}$ entnehmen. Der Stichprobenumfang je Saatstärkenvariante und Termin betrug 96 Pflanzen (je 16 Pflanzen aus 6 Wiederholungsparzellen).

Die Ermittlung der Abundanz und Verteilung der Eigelege und Larven von C. napi und $C$. pallidactylus erfolgte in allen drei Versuchsjahren an jeweils zwei Terminen im April und Mai (Tab. 10). Der 1. Termin der Probenahme lag am Höhepunkt der Eiablage von C. napi und $C$. pallidactylus, der 2. Termin direkt vor der Abwanderung der ersten Larven von C. napi und $C$. pallidactylus aus den Pflanzen. Pro Saatstärkenvariante und Termin wurden 90 Pflanzen (je 15 Pflanzen aus 6 Wiederholungsparzellen) untersucht.

Zur Erfassung der Eiablagepräferenz von C. napi und C. pallidactylus in Abhängigkeit vom Stängeldurchmesser wurde im April 2002 eine weitere Pflanzenprobe entnommen. Abweichend von der zuvor beschriebenen Vorgehensweise bei den Probenahmen wurden Pflanzen mit dicken und dünnen Stängeln gezielt aus der Mantelsaat gesucht und als Probenmaterial (je 40 Pflanzen) eingebracht.

Um die geeigneten Zeitpunkte für die Probenahmen festzulegen, wurden im Rapsbestand regelmäßig Pflanzen gesammelt und unter dem Binokular auf den Befall mit Eiern und Larven der Triebschädlinge kontrolliert. Die Pflanzenproben wurden bis zu ihrer Untersuchung im Kühlraum bei etwa $2{ }^{\circ} \mathrm{C}$ aufbewahrt. Die phänologischen Entwicklungsstadien der Rapspflan- 
zen wurden gemäß der BBCH-Skala (STAUß et al. 1994) bestimmt. Das herauspräparierte Tiermaterial wurde in 70 \%igem Alkohol konserviert.

Tab. 9: Probenahmetermine für die Ermittlung der Befallsdichte und -verteilung der Larven von $P$. chrysocephala an den Standorten Weendelsbreite und Otto-Hahn-Straße in den Versuchsjahren 1999 bis 2002

\begin{tabular}{|c|c|c|c|}
\hline Versuchsjahr & Standort & Termin & Untersuchungsparameter \\
\hline 1999 & Weendelsbreite & 11.11. & $\begin{array}{l}\text { 1. Larvenzahl pro Pflanze und pro } m^{2} \\
\text { 2. \% Larven in Endknospe, Spross, Seitentrieb- } \\
\text { knospen und Blättern } \\
\text { 3. Vertikale Verteilung der Larven in den Blatt- } \\
\text { etagen }\end{array}$ \\
\hline 2000 & Weendelsbreite & 20.10 & $\begin{array}{l}\text { 1. Vertikale Verteilung der Larven in den Blatt- } \\
\text { etagen }\end{array}$ \\
\hline 2000 & Weendelsbreite & 31.10. & $\begin{array}{l}\text { 1. Vertikale Verteilung der Larven in den Blatt- } \\
\text { etagen }\end{array}$ \\
\hline 2000 & Weendelsbreite & 16.11. & $\begin{array}{l}\text { 1. Larvenzahl pro Pflanze und pro } \mathrm{m}^{2} \\
\text { 2. \% Larven in Endknospe, Spross, Seitentrieb- } \\
\text { knospen und Blättern } \\
\text { 3. Vertikale Verteilung der Larven in den Blatt- } \\
\text { etagen }\end{array}$ \\
\hline 2001 & Weendelsbreite & 15.02. & $\begin{array}{l}\text { 1. \% Larven in Endknospe, Spross, Seitentrieb- } \\
\text { knospen und Blättern } \\
\text { 2. Vertikale Verteilung der Larven in den Blatt- } \\
\text { etagen } \\
\text { 3. Larvenzahl pro Pflanze und pro g pflanzlicher } \\
\text { Biomasse }\end{array}$ \\
\hline 2001 & Otto-Hahn-Straße & 12.11 & $\begin{array}{l}\text { 1. Vertikale Verteilung der Larven in den Blatt- } \\
\text { etagen }\end{array}$ \\
\hline 2001 & Otto-Hahn-Straße & 29.11. & $\begin{array}{l}\text { 1. Larvenzahl pro Pflanze und pro } \mathrm{m}^{2} \\
\text { 2. \% Larven in Endknospe, Spross, Seitentrieb- } \\
\text { knospen und Blättern } \\
\text { 3. Vertikale Verteilung der Larven in den Blatt- } \\
\text { etagen } \\
\text { 4. Larvenzahl pro Pflanze und pro g pflanzlicher } \\
\text { Biomasse }\end{array}$ \\
\hline 2002 & Otto-Hahn-Straße & 31.01. & $\begin{array}{l}\text { 1. \% Larven in Endknospe, Spross, Seitentrieb- } \\
\text { knospen und Blättern } \\
\text { 2. Vertikale Verteilung der Larven in den Blatt- } \\
\text { etagen } \\
\text { 3. Larvenzahl pro Pflanze und pro g pflanzlicher } \\
\text { Biomasse }\end{array}$ \\
\hline
\end{tabular}


Tab. 10: Termine der Entnahme von Pflanzenproben an den Standorten Weendelsbreite und Otto-Hahn-Straße zur Ermittlung der Befallsdichte und -verteilung der Eier und Larven von C. napi und C. pallidactylus in den Versuchsjahren 2000 bis 2002

\begin{tabular}{cll}
\hline Versuchsjahr & Standort & Termin \\
\hline 2000 & Weendelsbreite & 20.04. \\
2000 & Weendelsbreite & 12.05. \\
\hline 2001 & Weendelsbreite & 24.04. \\
2001 & Weendelsbreite & 25.05. \\
\hline 2002 & Otto-Hahn-Straße & 02.04. \\
2002 & Otto-Hahn-Straße & 17.04. \\
2002 & Otto-Hahn-Straße & 21.05. \\
\hline
\end{tabular}

\subsubsection{Ermittlung der Pflanzenlänge im Bestand}

Ergänzend zur Pflanzenbonitur wurde an je einem Termin im Frühjahr die Pflanzenlänge im Bestand erhoben. Die Vermessung der Pflanzen erfolgte entlang eines Transektes durch die Parzellen. Der Stichprobenumfang betrug je Saatstärke, Termin und Parzelle 32 Pflanzen. Die einzelnen Termine sind in Tabelle 11 dargestellt.

Tab. 11: Termine der Ermittlung von Pflanzenlänge im Freiland an den Standorten Weendelsbreite und Otto-Hahn-Straße in den Versuchsjahren 2000 bis 2002

\begin{tabular}{ccl}
\hline Versuchsjahr & Standort & Termin \\
\hline 2000 & Weendelsbreite & 29.03. \\
2001 & Weendelsbreite & 04.04. \\
2002 & Otto-Hahn-Straße & 28.03. \\
\hline
\end{tabular}

\subsubsection{Ermittlung der Biomasse der Rapspflanzen im Labor}

Für die Bestimmung der Biomasse wurden Pflanzen aus den im Abschnitt ,Pflanzenproben’ beschriebenen Probenahmen verwendet. Die Ermittlung der Biomasse im Labor erfolgte erst nach den Pflanzenuntersuchungen. Jede einzelne Pflanze wurde in einer Aluminium-Schale 24 $\mathrm{h}$ bei $105^{\circ} \mathrm{C}$ getrocknet und gewogen. Die Termine der Biomassenermittlung sind in Tabelle 12 zusammengefasst. 
Tab. 12: Termine der Ermittlung von Biomasse (Trockengewicht) im Labor an den Standorten Weendelsbreite und Otto-Hahn-Straße im Winter der Jahre 2001 und 2002

\begin{tabular}{ccc}
\hline Versuchsjahr & Standort & Termin \\
\hline 2001 & Weendelsbreite & 15.02. \\
2001 & Otto-Hahn-Straße & 29.11. \\
2002 & Otto-Hahn-Straße & 31.01. \\
\hline
\end{tabular}

\subsubsection{Ermittlung der Ertragsparameter}

Die Beerntung der Versuche wurde mit einem Parzellenmähdrescher mit einer Schnittbreite von 2,6 m als Kerndrusch durchgeführt. Die Länge der abgeernteten Kernparzellen betrug in den Erntejahren 2000, 2001 und 200215 m, 10 m bzw. 7,5 m. Die beernteten Teilflächen lagen außerhalb der intensiv beprobten Parzellenbereiche. Die geerntete Kornmenge pro Parzelle wurde eingesackt, beschriftet und gewogen. Nach dem Wiegen des Ernteguts wurde jedem Sack eine Stichprobe entnommen. Diese Kornproben dienten als Grundlage für die weitere Bestimmung von Kornfeuchte, Fremdbesatz und TKG. Zur Bestimmung der Kornfeuchte wurde diesen Grundproben je eine Stichprobe mit definiertem Volumen entnommen und gewogen, $24 \mathrm{~h}$ bei $105^{\circ} \mathrm{C}$ getrocknet und erneut gewogen. Nach Bereinigung des Fremdbesatzes wurde die Korntrockenmasse auf einen Feuchtegehalt von $9 \%$ umgerechnet. Für die Bestimmung des Fremdbesatzes und des TKG wurde der Rest der Grundproben zunächst bei Raumtemperatur bis zum Erreichen einer Gleichgewichtsfeuchte aufbewahrt. Vor der Bestimmung des Fremdbesatzes wurden die Proben gewogen, dann gereinigt und erneut gewogen. Für das TKG wurden dem gereinigten Material Stichproben entnommen und gewogen. Die Anzahl der Körner einer Stichprobe wurde mit einem Saatkornzählgerät erhoben. Anschließend wurde das TKG einer Stichprobe rechnerisch ermittelt. 


\section{Ergebnisse}

Bei der Darstellung der Ergebnisse wird zunächst auf die Dichten und Wachstumsparameter der Rapspflanzen als mögliche Einflussfaktoren auf die Aktivitäts- und Besiedlungsdichte, Eiablage und Larvenzahl der Triebschädlinge in den verschiedenen Saatstärkenvarianten eingegangen. Im Anschluss daran werden die Zuwanderung, die Siedlungs- und Aktivitätsdichte der Imagines und die Dichte und Verteilung der Präimaginalstadien auf verschiedene Pflanzenorgane erörtert. Zuletzt werden die Ergebnisse des Kornertrages/ha, des Einzelpflanzenertrages und des Tausendkorngewichtes dargestellt.

\subsection{Entwicklung der Pflanzendichten und Wachstumsparameter der Pflanzen in den Jahren 1999 bis 2002}

\subsubsection{Vorwinterentwicklung der Pflanzen in Abhängigkeit von der Witterung}

In den Anbauperioden 1999/00 und 2000/01 wurde die Vorwinterentwicklung der Pflanzen durch günstige Witterungsbedingungen im Herbst gefördert. Ein niederschlagsarmer August begünstigte eine rechtzeitige Aussaat bei optimalen Bodenverhältnissen. Ein infolge der Trockenheit zunächst etwas verzögertes Auflaufen der Saat im September 1999 wurde durch eine zügige Jugendentwicklung der Pflanzen ausgeglichen. Insbesondere die Hybridrapssorte zeigte im Herbst 2000 bei günstigen Witterungsbedingungen ein schnelles Wachstum und ging deutlich stärker entwickelt in die Vegetationsruhe als die Linienrapssorte im Herbst 1999.

Im Anbaujahr 2001/02 verlief die Vorwinterentwicklung der Hybridrapssorte verhaltener als im Jahr zuvor. Der September 2001 war durch nasskalte Witterung gekennzeichnet, wobei anhaltende Niederschläge zu einer starken Verschlämmung der Bodenoberfläche führten. Dies hatte insgesamt nicht nur einen sehr stark verzögerten Auflauf der Saat, sondern auch eine sehr zögerliche Jugendentwicklung der Pflanzen zur Folge. Überdurchschnittlich warme Temperaturen im Oktober 2001 begünstigten das Pflanzenwachstum und bewirkten einen mit der Linienrapssorte im Versuch 1999/00 vergleichbaren vorwinterlichen Entwicklungsstand der Pflanzen. 


\subsubsection{Pflanzendichten}

Die verschiedenen Saatstärken führten in allen drei Versuchsjahren zu entsprechend abgestuften, signifikant verschiedenen Pflanzendichten (Tab. 13, 14 und 15). Im Versuch 1999/00 wurden die Pflanzendichten in allen Varianten durch den Aufwuchs von im Boden überliegenden Ausfallraps erhöht. Ein signifikanter Rückgang der Pflanzendichte bis zum Frühjahr trat in den beiden ersten Versuchsjahren ausschließlich in der Saatvariante $90 \mathrm{Kö.} / \mathrm{m}^{2}$ auf, wobei die Pflanzendichte sowohl in den Insektizid-behandelten (Versuch 1999/00 und 2000/01) als auch in den unbehandelten Parzellen (Versuch 2000/01) verringert war. Im Versuchsjahr 2001/02 war hingegen bei beiden Saatstärken (30 und $60 \mathrm{Kö.} / \mathrm{m}^{2}$ ) eine statistisch gesicherte Reduktion der Pflanzendichte in den behandelten und unbehandelten Parzellen vom Herbst zum Frühjahr zu verzeichnen (Tab. 15). Durch die Insektizidbehandlung konnte nur bei der Saatstärke $90 \mathrm{Kö.} / \mathrm{m}^{2}$ im Versuch 2000/01 eine signifikante Verringerung der Pflanzenverluste von $19,4 \%$ in den unbehandelten auf $11,4 \%$ in den behandelten Parzellen erreicht werden (ANOVA, LSD-Test, $\mathrm{p}=0,027$ ).

Tab. 13: Mittlere Pflanzendichte ( \pm SE) bei unterschiedlichen Saatstärken am Standort Weendelsbreite im Versuch 1999/00

\begin{tabular}{|c|c|c|}
\hline \multirow{3}{*}{ Saatstärke } & \multicolumn{2}{|c|}{ Pflanzen $/ \mathrm{m}^{2}$} \\
\hline & Herbst 1999* & $\begin{array}{c}\text { Frühjahr } 2000 \\
\text { behandelt }\end{array}$ \\
\hline & $X_{m} \pm S E$ & $X_{m} \pm S E$ \\
\hline 30 Körner/m² & $40,8 \pm 1,9$ a $A$ & $37,4 \pm 2,8$ a $A$ \\
\hline 60 Körner/m² & $68,4 \pm 3,6$ b A & $65,9 \pm 2,8$ b A \\
\hline 90 Körner/m² & $102,6 \pm 4,0 \mathrm{c} A$ & $82,1 \pm 5,2$ c B \\
\hline
\end{tabular}

Tab. 14: Mittlere Pflanzendichte $( \pm \mathrm{SE})$ bei unterschiedlichen Saatstärken am Standort Weendelsbreite im Versuch 2000/01

\begin{tabular}{|c|c|c|c|}
\hline \multirow[b]{2}{*}{ Saatstärke } & \multicolumn{3}{|c|}{ Pflanzen $/ \mathrm{m}^{2}$} \\
\hline & $\begin{array}{l}\text { Herbst } 2000 \\
X_{m} \pm S E\end{array}$ & $\begin{array}{c}\text { Frühjahr } 2001 \\
\text { behandelt } \\
X_{m} \pm S E\end{array}$ & $\begin{array}{c}\text { Frühjahr } 2001 \\
\text { unbehandelt } \\
X_{m} \pm S E\end{array}$ \\
\hline 30 Körner/m² & $26,8 \pm 0,8$ a $A$ & $30,0 \pm 2,2$ a $A$ & $27,8 \pm 1,1$ a $A$ \\
\hline 60 Körner $/ \mathrm{m}^{2}$ & $49,3 \pm 2,0 \quad b \quad A$ & $54,1 \pm 1,6$ b $A$ & $47,1 \pm 1,6$ b A \\
\hline 90 Körner $/ \mathrm{m}^{2}$ & $86,2 \pm 1,2 \subset A$ & $76,4 \pm 1,9 \quad \mathrm{c} \quad \mathrm{B}$ & $69,5 \pm 1,8 \quad c \quad B$ \\
\hline
\end{tabular}

Verschiedene Klein- und Großbuchstaben indizieren signifikante Unterschiede zwischen den Saatstärken bzw. zwischen dem Herbst und den behandelten und unbehandelten Parzellen im Frühjahr, bei unabhängigen Stichproben: ANOVA, Tukey-Test, $p<0,001$, bei verbundenen Stichproben: $t-T e s t, p<0,01$ 
Tab. 15: Mittlere Pflanzendichte $( \pm \mathrm{SE})$ bei unterschiedlichen Saatstärken am Standort OttoHahn-Straße im Versuch 2001/02

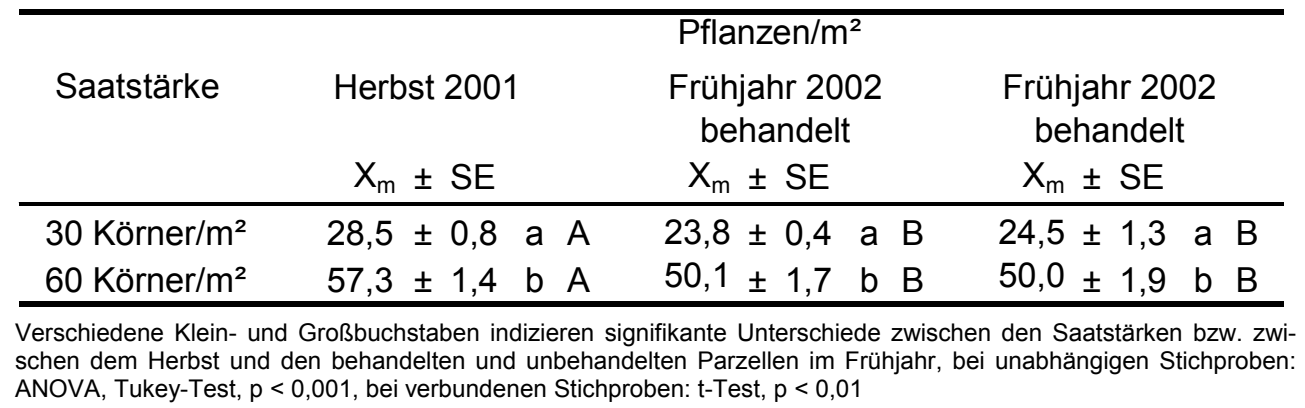

\subsubsection{Absterben der Triebe während des Winters}

Als Reaktion der Rapspflanzen auf den Larvenbefall von $P$. chrysocephala kann es zu einer Zerstörung des Vegetationspunktes am Haupttrieb kommen. Häufig sterben solche Pflanzen während des Winters vollständig ab. Zu Vegetationsbeginn im Frühjahr 2001 wurden in den Insektizid-unbehandelten Teilflächen allerdings Rapspflanzen beobachtet, an denen lediglich die Hauptsprossspitze abstarb, so dass der ungeschädigte Teil des Hauptsprosses und die intakten Seitentriebe das Überleben sicherten. Die Anzahl der Pflanzen ohne Hauptsprossspitze wurde erst nach der Ernte durch Untersuchung der Pflanzenstoppeln erhoben.

In Tabelle 16 ist die Anzahl der Pflanzen mit abgestorbenen Hauptsprossspitzen in den unterschiedlichen Saatstärken im Juli 2001 aufgeführt. Bei den Saatstärken 60 und 90 Kö./m² wiesen deutlich mehr Pflanzen eine abgestorbene Sprossspitze auf $\left(7,2 \mathrm{bzw} .7,3 \mathrm{Pfl} . / \mathrm{m}^{2}\right)$ als bei der Saatstärke $30 \mathrm{Kö.} / \mathrm{m}^{2}$ (4,2 Pfl. $\left./ \mathrm{m}^{2}\right)$. Diese Unterschiede ließen sich statistisch nicht absichern (Tab. 16). Bezogen auf die Pflanzendichte $/ \mathrm{m}^{2}$ in den einzelnen Saatstärkevarianten war der Anteil der Pflanzen $/ \mathrm{m}^{2}$ mit fehlenden Hauptsprossen zur Ernte bei der Saatstärke 30 Kö. $/ \mathrm{m}^{2}$ mit 18,9 \% gegenüber 16,8 \% bei der Saatstärke $60 \mathrm{Kö.} / \mathrm{m}^{2}$ und 12,0 \% bei der Saatstärke $90 \mathrm{Kö.} / \mathrm{m}^{2}$ am höchsten. Die Unterschiede zwischen den Saatstärken waren aber nicht signifikant. 
Tab. 16: Mittlere Anzahl Pflanzen mit abgestorbenen Haupttrieben ( \pm SE) bei unterschiedlichen Saatstärken am Standort Weendelsbreite im Juli 2001

\begin{tabular}{lcr}
\hline Saatstärke & \multicolumn{2}{c}{$\begin{array}{c}\text { Pflanzen } / \mathrm{m}^{2} \\
\text { absolut }\end{array}$} \\
& $\mathrm{X}_{\mathrm{m}} \pm \mathrm{SE}$ & $\mathrm{X}_{\mathrm{m}} \pm \mathrm{SE}$ \\
\hline $30 \mathrm{Körner} / \mathrm{m}^{2}$ & $4,2 \pm 0,9$ a & $18,9 \pm 5,0$ a \\
$60 \mathrm{Körner} / \mathrm{m}^{2}$ & $7,2 \pm 1,2$ a & $16,8 \pm 2,4$ a \\
$90 \mathrm{Körner} / \mathrm{m}^{2}$ & $7,3 \pm 1,0$ a & $12,0 \pm 1,8$ a \\
\hline Verschiedene Kleinbuchstaben indizieren signifikante Unterschiede zwischen \\
den Saatstärken, ANOVA, Tukey-Test, $\mathrm{p}<0,05$
\end{tabular}

Zwischen dem Vegetationsbeginn im Frühjahr und dem Erntetermin ergab sich allgemein eine Reduktion der Pflanzenzahl (Tab. 17). Der höchste Pflanzenverlust war bei der Saatstärke 90 Kö. $/ \mathrm{m}^{2}\left(8,4 \mathrm{Pfl} . / \mathrm{m}^{2}\right)$ festzustellen. In den Varianten mit 30 und $60 \mathrm{Kö.} / \mathrm{m}^{2}$ waren die Pflanzenverluste mit 4,4 bzw. 5,0 Pflanzen $/ \mathrm{m}^{2}$ nur etwa halb so hoch wie bei der Variante $90 \mathrm{Kö.} / \mathrm{m}^{2}$. Bei den Saatvarianten 30 und $90 \mathrm{Kö.} / \mathrm{m}^{2}$ sank die Pflanzendichte im Zeitraum April bis Juli signifikant ab. Durch diese Pflanzenverluste könnte der Anteil von Pflanzen ohne Haupttriebe bei allen Saatstärken, insbesondere jedoch bei der Saatstärke $90 \mathrm{Kö.} / \mathrm{m}^{2}$, bei der Stoppelzählung unterschätzt worden sein.

Tab. 17: Mittlere Pflanzendichte ( \pm SE) bei unterschiedlichen Saatstärken am Standort Weendelsbreite im April und Juli 2001

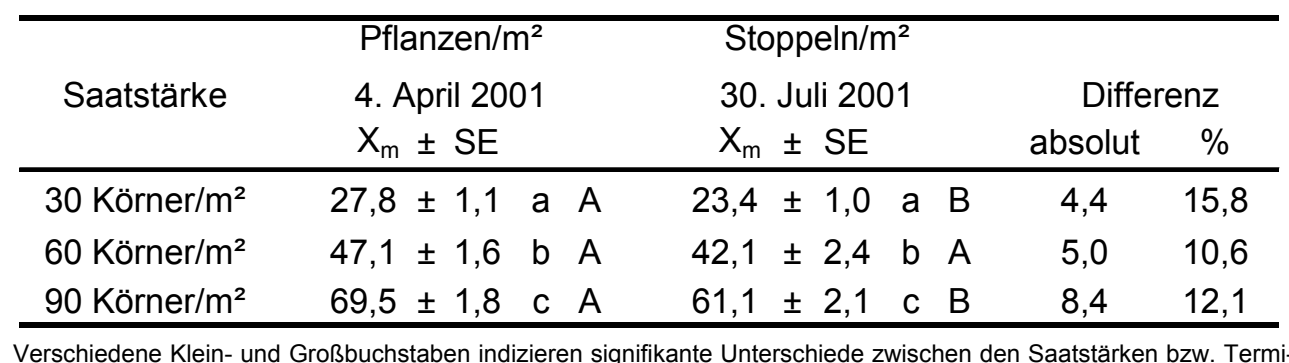

nen, bei unabhängigen Stichproben: ANOVA, Tukey-Test, $p<0,001$, bei verbundsen Stichproben: t-test, $p<0,05$

\subsubsection{Entwicklung der Pflanzenarchitektur}

\subsubsection{Sprossdurchmesser, Anzahl der Blätter und Seitentriebe sowie Sprosslänge}

In Tabelle 18 ist der Sprossdurchmesser am Wurzelhals und in $50 \mathrm{~cm}$ Höhe, die Anzahl der Blätter und Seitentriebe sowie die Sprosslänge der Rapspflanzen an drei verschiedenen Probeterminen im November (Tab. 18a), April (Tab. 18b) bzw. Mai (Tab. 18c) in den Anbaujahren 
1999/00 bis 2001/02 zusammengestellt. In Abhängigkeit von der Standraumzumessung in den einzelnen Saatstärkevarianten kam es zu deutlichen Unterschieden bei den Wachstumsparametern der Pflanzen. Die Saatstärke $30 \mathrm{Kö.} / \mathrm{m}^{2}$ führte in allen Versuchen im Vergleich zu den Saatstärken 60 und $90 \mathrm{Kö.} / \mathrm{m}^{2}$ zu wesentlich kräftiger entwickelten Einzelpflanzen: Mit abnehmender Saatstärke stiegen der Sprossdurchmesser am Wurzelhals und in $50 \mathrm{~cm}$ Höhe sowie die Anzahl der Blätter und Seitentriebe signifikant an. Ein signifikanter Anstieg der Sprosslänge war nur im November 1999, im April 2001 und 2002 sowie im Mai 2000 und $2001 \mathrm{zu}$ beobachten. Der Vergleich der Saatstärken zeigte auch, das die Entwicklung der oben genannten Pflanzenparameter bei den beiden hohen Saatstärken 60 und $90 \mathrm{Kö.} / \mathrm{m}^{2}$ nicht immer signifikant verschieden war; dies machte sich insbesondere bei der Linienrapssorte im Versuch 1999/00 bemerkbar.

Die im Mai 2002 ermittelte Anzahl der schotentragenden Seitentriebe war bei der Saatstärke $30 \mathrm{Kö.} / \mathrm{m}^{2}$ mit 10,0 Seitentrieben/Pflanze signifikant höher als bei der Saatstärke $60 \mathrm{Kö} . / \mathrm{m}^{2}$ mit 5,5 Trieben/Pflanze (Mann-Whitney U-Test, $\mathrm{p}=0,009$ ). Die Anzahl der verkümmerten Seitentriebe betrug bei den Saatstärken 30 und 60 Kö./m² 4,3 bzw. 4,8 Triebe/Pflanze. Diese Differenz ließ sich statistisch nicht absichern (Mann-Whitney U-Test, $p=0,655$ ).

Tab. 18a: Mittelwerte der Wachstumsparameter der Pflanzen ( \pm SE) unterschiedlicher Saatstärken im November 1999 und 2000 am Standort Weendelsbreite und im November 2001 am Standort Otto-Hahn-Straße (BBCH 15-18)

\begin{tabular}{|c|c|c|c|c|c|c|c|}
\hline \multirow[t]{2}{*}{ Jahr } & \multirow[t]{2}{*}{ Saatstärke } & \multicolumn{2}{|c|}{$\begin{array}{c}\text { Sprossdurch- } \\
\text { messer am } \\
\text { Wurzelhals [mm] }\end{array}$} & \multicolumn{2}{|l|}{$\begin{array}{l}\text { Anzahl } \\
\text { Blätter }\end{array}$} & \multicolumn{2}{|l|}{$\begin{array}{l}\text { Sprosslänge } \\
{[\mathrm{mm}]}\end{array}$} \\
\hline & & $X_{m} \pm S E$ & & $\mathrm{X}_{\mathrm{m}} \pm \mathrm{SE}$ & & $X_{m} \pm S E$ & \\
\hline \multirow[t]{3}{*}{1999} & 30 Körner $/ \mathrm{m}^{2}$ & $10,2 \pm 0,3$ & $\bar{a}$ & $6,9 \pm 0,1$ & $\bar{a}$ & $18,6 \pm 0,6$ & $\bar{a}$ \\
\hline & 60 Körner/m² & $6,9 \pm 0,7$ & b & $5,4 \pm 0,3$ & $\mathrm{~b}$ & $13,7 \pm 0,8$ & $b$ \\
\hline & 90 Körner/m² & $6,6 \pm 0,6$ & $b$ & $5,1 \pm 0,2$ & $b$ & $12,9 \pm 0,8$ & $b$ \\
\hline \multirow[t]{3}{*}{2000} & 30 Körner/m² & $13,8 \pm 0,4$ & $a$ & $11,0 \pm 0,3$ & a & $29,8 \pm 2,2$ & a \\
\hline & 60 Körner/m² & $10,5 \pm 0,4$ & $b$ & $8,4 \pm 0,2$ & $\mathrm{~b}$ & $29,2 \pm 3,3$ & a \\
\hline & 90 Körner/m² & $8,3 \pm 0,2$ & c & $7,6 \pm 0,1$ & b & $25,8 \pm 1,8$ & a \\
\hline \multirow[t]{2}{*}{2001} & 30 Körner/m² & $10,7 \pm 0,2$ & $a$ & $10,3 \pm 0,1$ & a & $16,4 \pm 0,6$ & $a$ \\
\hline & 60 Körner/m² & $7,4 \pm 0,2$ & $b$ & $8,2 \pm 0,1$ & $\mathrm{~b}$ & $16,3 \pm 1,0$ & a \\
\hline
\end{tabular}

Verschiedene Buchstaben indizieren signifikante Unterschiede zwischen den Saatstärken, ANOVA, Tukey-Test, $p<0,05$ 
Tab. 18b: Mittelwerte der Wachstumsparameter der Pflanzen ( \pm SE) unterschiedlicher Saatstärken im April 2000 und 2001 am Standort Weendelsbreite und im April 2002 am Standort Otto-Hahn-Straße (BBCH 55-59)

\begin{tabular}{|c|c|c|c|c|c|c|c|c|c|}
\hline Jahr & Saatstärke & \multicolumn{2}{|l|}{$\begin{array}{c}\text { Sprossdurch- } \\
\text { messer am } \\
\text { Wurzelhals } \\
\text { [mm] }\end{array}$} & \multicolumn{2}{|l|}{$\begin{array}{l}\text { Anzahl } \\
\text { Blätter }\end{array}$} & \multicolumn{2}{|l|}{$\begin{array}{c}\text { Anzahl } \\
\text { primärer } \\
\text { Seitentriebe }\end{array}$} & \multicolumn{2}{|l|}{$\begin{array}{l}\text { Spross- } \\
\text { länge } \\
\text { [cm] }\end{array}$} \\
\hline \multirow[t]{3}{*}{2000} & $30 \mathrm{Körner} / \mathrm{m}^{2}$ & $14,9 \pm 1,3$ & $a$ & $17,5 \pm 0,5$ & a & $10,6 \pm 1,1$ & $a$ & $65,9 \pm 4,4$ & a \\
\hline & $60 \mathrm{Körner} / \mathrm{m}^{2}$ & $12,1 \pm 0,3$ & $a$ & $15,9 \pm 0,4$ & b & $6,3 \pm 0,4$ & b & $62,6 \pm 1,6$ & a \\
\hline & 90 Körner/m² & $10,3 \pm 0,6$ & b & $13,7 \pm 0,7$ & c & $3,7 \pm 0,9$ & b & $56,7 \pm 2,7$ & a \\
\hline \multirow[t]{3}{*}{2001} & 30 Körner/m² & $18,4 \pm 0,3$ & a & $24,1 \pm 1,1$ & a & $14,0 \pm 0,6$ & a & $62,2 \pm 0,8$ & $\mathrm{a}$ \\
\hline & 60 Körner $/ \mathrm{m}^{2}$ & $14,2 \pm 0,3$ & b & $20,3 \pm 1,0$ & b & $10,0 \pm 0,5$ & b & $55,6 \pm 0,7$ & $\mathrm{~b}$ \\
\hline & 90 Körner/m² & $10,9 \pm 0,2$ & c & $18,3 \pm 0,8$ & $b$ & $6,9 \pm 0,4$ & c & $45,6 \pm 0,8$ & C \\
\hline \multirow[t]{2}{*}{2002} & 30 Körner/m² & $16,6 \pm 0,3$ & a & $21,1 \pm 0,3$ & a & $12,4 \pm 0,7$ & a & $56,7 \pm 1,6$ & a \\
\hline & $60 \mathrm{Körner} / \mathrm{m}^{2}$ & $12,6 \pm 0,2$ & b & $19,1 \pm 0,2$ & b & $6,3 \pm 0,4$ & b & $49,7 \pm 1,6$ & b \\
\hline
\end{tabular}

Verschiedene Buchstaben indizieren signifikante Unterschiede zwischen den Saatstärken, 2000 und 2001: ANOVA, Tukey-Test, 2002: MannWhitney U-Test, $p<0,05$

Tab. 18c: Mittelwerte der Wachstumsparameter der Pflanzen ( \pm SE) unterschiedlicher Saatstärken im Mai 2000 und 2001 am Standort Weendelsbreite und im Mai 2002 am Standort Otto-Hahn-Straße (BBCH 67-69)

\begin{tabular}{|c|c|c|c|c|c|c|}
\hline Jahr & Saatstärke & $\begin{array}{c}\text { Spross- } \\
\text { durchmesser } \\
\text { am Wurzelhals } \\
{[\mathrm{mm}]} \\
\mathrm{X}_{\mathrm{m}} \pm \mathrm{SE}\end{array}$ & $\begin{array}{c}\text { Spross- } \\
\text { durchmesser } \\
\text { in } 50 \mathrm{~cm} \\
\text { Höhe }[\mathrm{mm}] \\
\mathrm{X}_{\mathrm{m}} \pm \mathrm{SE}\end{array}$ & $\begin{array}{c}\text { Anzahl } \\
\text { primärer } \\
\text { Seitentriebe }\end{array}$ & $\begin{array}{c}\text { Anzahl } \\
\text { sekundärer } \\
\text { Seitentriebe } \\
\mathrm{X}_{\mathrm{m}} \pm \mathrm{SE}\end{array}$ & $\begin{array}{l}\text { Spross- } \\
\text { länge } \\
{[\mathrm{cm}]} \\
X_{\mathrm{m}} \pm \mathrm{SE}\end{array}$ \\
\hline \multirow[t]{3}{*}{2000} & 30 Körner/m² & $13,8 \pm 0,4$ a & $8,9 \pm 0,3 a$ & $15,6 \pm 0,4 a$ & $4,7 \pm 0,4$ a & $128,9 \pm 3,4 a$ \\
\hline & 60 Körner/m² & $11,5 \pm 0,4 b$ & $7,5 \pm 0,3 b$ & $12,7 \pm 0,6 b$ & $1,9 \pm 0,3 b$ & $118,2 \pm 2,9 b$ \\
\hline & 90 Körner/m² & $9,1 \pm 0,2 c$ & $5,8 \pm 0,2 c$ & $9,6 \pm 0,5 \mathrm{c}$ & $0,3 \pm 0,1 \mathrm{c}$ & $109,0 \pm 1,5 b$ \\
\hline \multirow[t]{3}{*}{2001} & 30 Körner/m² & $19,0 \pm 0,4 a$ & $10,2 \pm 0,3 a$ & $14,4 \pm 0,3 a$ & $16,9 \pm 1,6 a$ & $143,8 \pm 1,4$ \\
\hline & 60 Körner/m² & $13,8 \pm 0,4 b$ & $7,6 \pm 0,2 b$ & $11,1 \pm 0,2 b$ & $8,9 \pm 0,4 b$ & $128,7 \pm 3,6$ \\
\hline & 90 Körner/m² & $10,4 \pm 0,3 \mathrm{c}$ & $6,2 \pm 0,2 c$ & $8,8 \pm 0,2 c$ & $2,6 \pm 0,3 c$ & $122,5 \pm 3,5 b$ \\
\hline \multirow[t]{2}{*}{2002} & 30 Körner/m² & $18,4 \pm 0,6 a$ & $12,3 \pm 0,4 a$ & $16,5 \pm 0,8 a$ & $31,5 \pm 1,4 a$ & $159,3 \pm 5,3$ \\
\hline & 60 Körner $/ \mathrm{m}^{2}$ & $13,8 \pm 0,2 b$ & $11,2 \pm 0,2 b$ & $11,1 \pm 0,8 b$ & $13,1 \pm 2,1 b$ & $144,8 \pm 5,5$ \\
\hline
\end{tabular}

Verschiedene Buchstaben indizieren signifikante Unterschiede zwischen den Saatstärken, 2000 und 2001: ANOVA, Tukey-Test, 2002: MannWhitney U-Test, $p<0,05$

\subsubsection{Seitensprossknospen}

Im Versuch 2000/01 konnten deutliche Auswirkungen der Standraumverhältnisse auf die vorwinterliche Entwicklung der Seitensprossknospen festgestellt werden, die jedoch nicht quanti- 
fiziert wurden. Die Pflanzen der Saatstärke $30 \mathrm{Kö.} / \mathrm{m}^{2}$ besaßen im Vergleich zu denen der Saatstärken 60 und $90 \mathrm{Kö.} / \mathrm{m}^{2}$ weiter entwickelte Seitensprossknospen mit sichtbarem Längenund Dickenwachstum. Dies führte zu einem höheren Befall der Seitentriebknospen durch die Larven von P. chrysocephala im Februar 2001 (Kap. 3.2.9.2) und zu einer frühen Ausbildung der Seitentriebe im April 2001 (Kap. 3.1.4.4).

\subsubsection{Pflanzenhöhe im Bestand}

Tabelle 19 zeigt Ende März/Anfang April im Versuchsfeld ermittelte Höhe der Pflanzen. Die kräftigen Pflanzen der Saatstärke $30 \mathrm{Kö.} / \mathrm{m}^{2}$ übertrafen die schwächer entwickelten Pflanzen der Saatstärken 60 und $90 \mathrm{Kö.} / \mathrm{m}^{2}$ in ihrem Längenwachstum deutlich: Mit zunehmender Saatstärke nahm die Länge der Pflanzen infolge der verzögerten Entwicklung im Herbst in allen Versuchen signifikant ab.

Tab. 19: Mittlere Höhe der Pflanzen ( \pm SE) bei unterschiedlichen Saatstärken im März/April 2000 und 2001 am Standort Weendelsbreite und im März 2002 am Standort OttoHahn-Straße (BBCH 50-51)

\begin{tabular}{|c|c|c|c|c|c|}
\hline \multirow[t]{2}{*}{ Jahr } & \multirow[t]{2}{*}{ Saatstärke } & \multicolumn{4}{|c|}{$\begin{array}{l}\text { Pflanzenhöhe } \\
\text { [cm] }\end{array}$} \\
\hline & & $X_{m}$ & \pm & SE & \\
\hline \multirow[t]{3}{*}{2000} & 30 Körner/m² & 16,6 & \pm & 1,3 & a \\
\hline & 60 Körner/m² & 11,6 & \pm & 0,8 & $b$ \\
\hline & 90 Körner/m² & 8,9 & \pm & 0,4 & c \\
\hline \multirow[t]{3}{*}{2001} & 30 Körner/m² & 21,8 & \pm & 0,6 & $a$ \\
\hline & 60 Körner/m² & 16,6 & \pm & 0,5 & $D$ \\
\hline & 90 Körner/m² & 12,4 & \pm & 0,3 & c \\
\hline \multirow[t]{2}{*}{2002} & 30 Körner/m² & 14,6 & \pm & 0,5 & 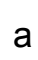 \\
\hline & 60 Körner/m² & 12,3 & \pm & 0,5 & b \\
\hline
\end{tabular}

Verschiedene Buchstaben indizieren signifikante Unterschiede zwischen den Saatstärken, 2000 und 2001: ANOVA, Tukey-Test, 2002: MannWhitney U-Test, $p<0,05$

\subsubsection{Längen- und Dickenwachstum der Seitentriebe}

Im April 2000 und 2001 wurden die Pflanzen durch Einteilung in 8 Klassen mit unterschiedlicher Länge der Seitentriebe differenziert (Tab. 20). Die Ergebnisse lassen erkennen, dass das Längenwachstum der Seitentriebe in beiden Versuchen mit zunehmender Saatstärke signifikant abnahm. Die im April 2001 und 2002 durch Messung exakter erfassten Längen und 
Durchmesser der Seitentriebe in $20 \mathrm{~cm}$ und $40 \mathrm{~cm}$ Höhe bzw. in drei definierten Abschnitten der Hauptsprossachse $(0-20 \mathrm{~cm}, 20-40 \mathrm{~cm}$ und über $40 \mathrm{~cm})$ zeigten ebenfalls einen starken Einfluss der Saatstärke: Bei den Saatstärken 60 und 90 Kö. $/ \mathrm{m}^{2}$ wurden signifikant geringere Längen und Durchmesser der Seitentriebe nachgewiesen als bei der Saatstärke $30 \mathrm{Kö.} / \mathrm{m}^{2}$ (Abb. 7 und 8). Die mittleren Längen und Durchmesser der Seitentriebe der Gesamtpflanzen sind in Tabelle 21 angegeben. Stellt man die in den Versuchen 2001 und 2002 gemessenen Seitentrieblängen den Trieblängenklassen in den Versuchen 2000 und 2001 aus Tabelle 20 gegenüber, so ist deutlich zu erkennen, dass die Entwicklung der Seitentriebe bei den Hybridrapspflanzen im Versuch 2001 insgesamt schon weiter fortgeschritten war als bei den Pflanzen der Linien- und Hybridrapssorte in den Versuchen 2000 bzw. 2002.

Tab. 20: Mittlere Länge der Seitentriebe $( \pm$ SE) an Pflanzen verschiedener Saatstärken im April 2000 und 2001 (BBCH 55-59) am Standort Weendelsbreite

\begin{tabular}{|c|c|c|c|c|c|c|}
\hline \multirow[t]{2}{*}{ Jahr } & \multirow[t]{2}{*}{ Saatstärke } & \multicolumn{5}{|c|}{$\begin{array}{c}\text { Mittelwert der } \\
\text { Klassifizierung der } \\
\text { Seitentrieblänge* }^{*}\end{array}$} \\
\hline & & $X_{m}$ & \pm & SE & & \\
\hline \multirow[t]{3}{*}{2000} & 30 Körner/m² & 5,2 & \pm & 0,6 & a & $A$ \\
\hline & 60 Körner/m² & 3,5 & \pm & 0,1 & $b$ & $A$ \\
\hline & 90 Körner/m² & 2,8 & \pm & 0,1 & $\mathrm{C}$ & $A$ \\
\hline \multirow[t]{3}{*}{2001} & 30 Körner/m² & 5,4 & \pm & 0,3 & $a$ & $A$ \\
\hline & 60 Körner/m² & 4,0 & \pm & 0,1 & $b$ & $B$ \\
\hline & 90 Körner/m² & 3,5 & \pm & 0,1 & $\mathrm{~b}$ & B \\
\hline
\end{tabular}

Tab. 21: Mittlere Länge und Durchmesser der Seitentriebe ( \pm SE) an Pflanzen unterschiedlicher Saatstärken im April 2001 und 2002 (BBCH 55-59) an den Standorten Weendelsbreite bzw. Otto-Hahn-Straße

\begin{tabular}{|c|c|c|c|c|c|c|c|c|c|}
\hline \multirow[t]{2}{*}{ Jahr } & \multirow[t]{2}{*}{ Saatstärke } & \multicolumn{4}{|c|}{$\begin{array}{l}\text { Länge der } \\
\text { Seitentriebe } \\
{[\mathrm{cm}]}\end{array}$} & \multicolumn{4}{|c|}{$\begin{array}{l}\text { Durchmesser der } \\
\text { Seitentriebe } \\
{[\mathrm{mm}]}\end{array}$} \\
\hline & & $\mathrm{X}_{\mathrm{m}}$ & \pm & SE & & $X_{m}$ & \pm & SE & \\
\hline \multirow[t]{3}{*}{2001} & 30 Körner/m² & 4,6 & \pm & 0,3 & a & 2,7 & \pm & 0,0 & a \\
\hline & 60 Körner/m² & 2,5 & \pm & 0,2 & $b$ & 2,1 & \pm & 0,1 & $b$ \\
\hline & 90 Körner/m² & 1,9 & \pm & 0,2 & $\mathrm{~b}$ & 1,8 & \pm & 0,1 & C \\
\hline \multirow[t]{2}{*}{2002} & 30 Körner/m² & 2,8 & \pm & 0,2 & a & 2,1 & \pm & 0,1 & a \\
\hline & 60 Körner/m² & 2,1 & \pm & 0,2 & $b$ & 1,9 & \pm & 0,0 & $b$ \\
\hline
\end{tabular}

Verschiedene Buchstaben indizieren signifikante Unterschiede zwischen den Saatstärken, 2001: ANOVA, Tukey-Test, 2002: Mann-Whitney U-Test, $p<0,05$ 

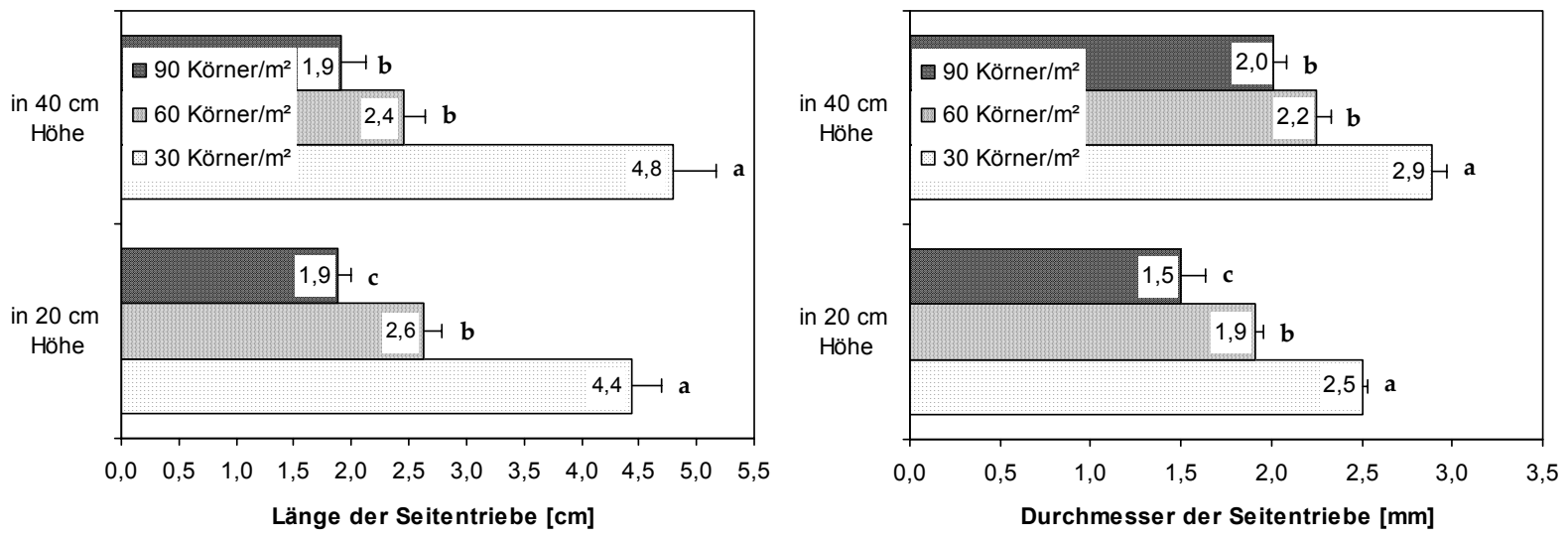

Abb. 7: Mittlere Länge und Durchmesser der Seitentriebe ( \pm SE) in 20 und $40 \mathrm{~cm}$ Höhe an Pflanzen verschiedener Saatstärken im April 2001 (BBCH 55-59) am Standort OttoHahn-Straße (Verschiedene Buchstaben indizieren signifikante Unterschiede zwischen den Saatstärken, ANOVA, Tukey-Test, $\mathrm{p}<0,05$ )
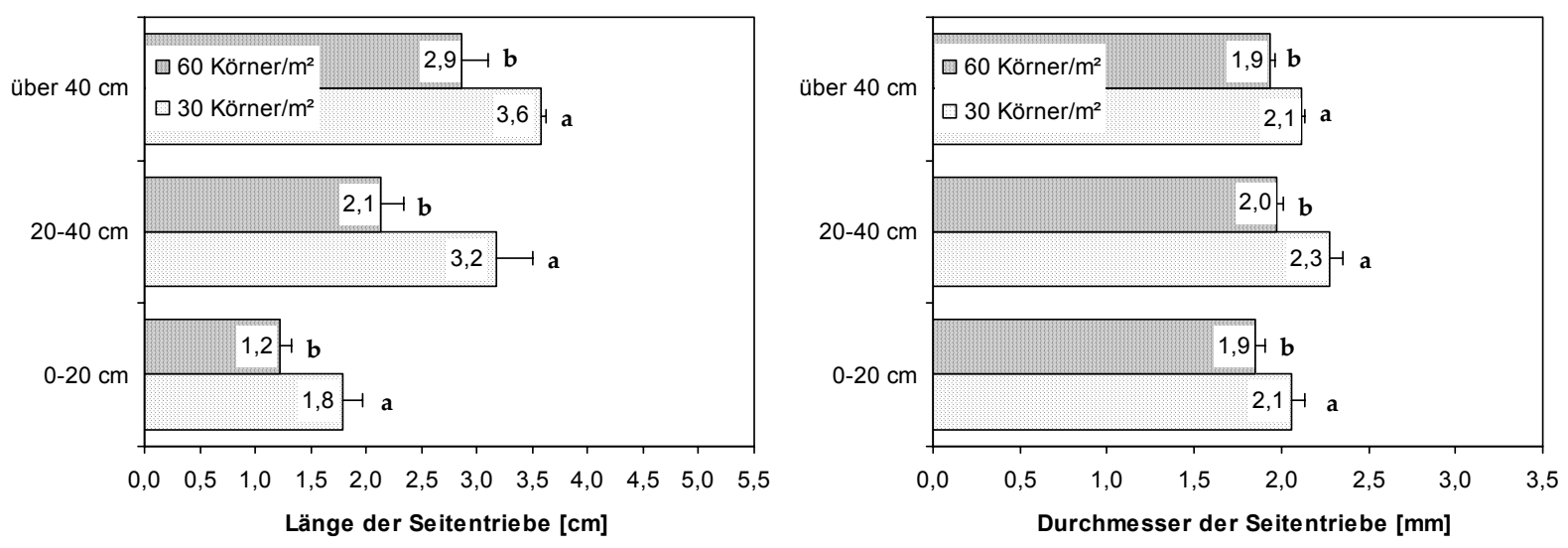

Abb. 8: Mittlere Länge und Durchmesser der Seitentriebe ( \pm SE) in drei Abschnitten der Hauptsprossachse an Pflanzen verschiedener Saatstärken im April 2002 (BBCH 5559) am Standort Otto-Hahn-Straße (Verschiedene Buchstaben indizieren signifikante Unterschiede zwischen den Saatstärken, Mann-Whitney U-Test, $\mathrm{p}<0,05$ )

\subsubsection{Biomasse-Produktion}

Analog zur Einzelpflanzenentwicklung stieg die Biomasse-Produktion der Pflanzen mit abnehmender Saatstärke an (Tab. 22). Zu allen drei Terminen waren deutliche Unterschiede in der Trockenmasse-Produktion zwischen den Saatstärken zu beobachten, lediglich im Februar 2001 war die Differenz zwischen den Saatstärken 60 und $90 \mathrm{Kö.} / \mathrm{m}^{2}$ nicht signifikant. Von November 2001 bis Januar 2002 sank die Trockenmasse bei der Saatstärke $30 \mathrm{Kö.} / \mathrm{m}^{2}$ wesentlich stärker ab als bei der Saatstärke $60 \mathrm{Kö.} / \mathrm{m}^{2}$. 
Als Folge unterschiedlicher Aussaat- und Wachstumsbedingungen in den Versuchen 2000/01 und 2001/02 wiesen die Pflanzen der Saatstärken 30 und $60 \mathrm{Kö.} / \mathrm{m}^{2}$ im Februar 2001 eine um das 1,7-fache bzw. 1,4-fache höhere Trockenmasse auf als die Pflanzen der entsprechenden Saatstärken im Januar 2002 (vgl. Kap. 3.1.1).

Tab. 22: Mittlere Trockenmasse der Pflanzen $( \pm \mathrm{SE})$ verschiedener Saatstärken im Versuch 2000/01 und 2001/02 an den Standorten Weendelsbreite bzw. Otto-Hahn-Straße

\begin{tabular}{|c|c|c|c|c|}
\hline \multirow{4}{*}{ Saatstärke } & \multicolumn{4}{|c|}{ TrM [g/Pflanze] } \\
\hline & \multirow{2}{*}{$\begin{array}{c}\text { Versuch } 2000 / 01 \\
15.02 .01\end{array}$} & \multicolumn{3}{|c|}{ Versuch 2001/02 } \\
\hline & & 29.11 .01 & 31.01 .02 & \\
\hline & $\mathrm{X}_{\mathrm{m}} \pm \mathrm{SE}$ & $\mathrm{X}_{\mathrm{m}} \pm \mathrm{SE}$ & $\mathrm{X}_{\mathrm{m}} \pm \mathrm{SE}$ & \\
\hline 30 Körner/m² & $8,2 \pm 0,8 \quad a$ & $6,7 \pm 0,4$ a $A$ & $4,9 \pm 0,3$ & $B$ \\
\hline 60 Körner/m² & $3,9 \pm 0,4 b$ & $2,9 \pm 0,1 \quad b \quad A$ & $2,8 \pm 0,2$ & $A$ \\
\hline 90 Körner/m² & $2,2 \pm 0,2 b$ & - & - & \\
\hline
\end{tabular}

Verschiedene Klein- und Großbuchstaben indizieren signifikante Unterschiede zwischen den Saatstärken bzw. Jahreszeiten, bei unabhängigen Stichproben: 2001: ANOVA, Tukey-Test, 2002: Mann-Whitney U-Test, $p<0,05$, bei verbundenen Stichproben: $t-T e s t, p<0,05$

\subsubsection{Beziehung zwischen den Wachstumsparametern der Pflanzen und der Pflanzen- dichte}

Zwischen den Wachstumsparametern der Pflanzen und der Pflanzendichte ergaben sich in den Monaten November (Tab. 23a), April (Tab. 23b) und Mai (Tab. 23c) der drei Versuchsjahre mit nur wenigen Ausnahmen signifikante und eng korrelierte negative Zusammenhänge. Lediglich für die Sprosslänge im November 2000 und 2001 und im Mai 2002 sowie für den Sprossdurchmesser in $50 \mathrm{~cm}$ Höhe im Mai 2002 wurden keine signifikanten Beziehungen zur Pflanzendichte nachgewiesen. Es muss dabei jedoch berücksichtigt werden, dass in den Versuchen 2000/01 und 2001/02 gegenüber dem Versuch 1999/00 ein Wechsel von der Linienzur Hybridrapssorte vorgenommen wurde und der Befallsdruck mit C. napi im Frühjahr 2002 (Kap. 3.3.3) relativ hoch war. Darüber hinaus ergaben sich bei der Hybridrapssorte in den Versuchen 2000/01 und 2001/02 insgesamt engere Korrelationen als bei der Liniensorte im Versuch 1999/00. 
Tab. 23a: Korrelationen zwischen den Wachstumsparametern der Pflanzen und der Pflanzendichte im November 1999 und 2000 am Standort Weendelsbreite und im November 2001 am Standort Otto-Hahn-Straße

\begin{tabular}{|c|c|c|c|c|c|}
\hline $\begin{array}{c}\text { Korrelier } \\
\text { Merkm }\end{array}$ & ande & Jahr & $r$ & $p$ & $\mathrm{~B}[\%]$ \\
\hline $\begin{array}{l}\text { Sprossdurchmesser } \\
\text { am Wurzelhals }\end{array}$ & - Pflanzendichte & $\begin{array}{l}1999 \\
2000 \\
2001\end{array}$ & $\begin{array}{l}-0,69 \\
-0,92 \\
-0,91\end{array}$ & $\begin{array}{l}0,004 \\
0,000 \\
0,000\end{array}$ & $\begin{array}{l}48,0 \\
85,2 \\
82,0\end{array}$ \\
\hline Anzahl Blätter & - Pflanzendichte & $\begin{array}{l}1999 \\
2000 \\
2001\end{array}$ & $\begin{array}{l}-0,73 \\
-0,85 \\
-0,95\end{array}$ & $\begin{array}{l}0,001 \\
0,000 \\
0,000\end{array}$ & $\begin{array}{l}53,9 \\
71,6 \\
91,2\end{array}$ \\
\hline pfl. Trockenmasse & - Pflanzendichte & $\begin{array}{l}1999 \\
2000 \\
2001\end{array}$ & $\begin{array}{c}- \\
- \\
-0,91\end{array}$ & 0,000 & 83,7 \\
\hline Sprosslänge & - Pflanzendichte & $\begin{array}{l}1999 \\
2000 \\
2001 \\
\end{array}$ & $\begin{array}{l}-0,77 \\
-0,24 \\
+0,07\end{array}$ & $\begin{array}{l}0,000 \\
0,336 \\
0,812\end{array}$ & $\begin{array}{c}60,0 \\
5,8 \\
0,6 \\
\end{array}$ \\
\hline
\end{tabular}

Tab. 23b: Korrelationen zwischen den Wachstumsparametern der Pflanzen und der Pflanzendichte im April 2000 und 2001 am Standort Weendelsbreite und im April 2002 am Standort Otto-Hahn-Straße

\begin{tabular}{|c|c|c|c|c|c|}
\hline \multicolumn{2}{|c|}{$\begin{array}{c}\text { Korrelierende } \\
\text { Merkmale }\end{array}$} & Jahr & $r$ & $\mathrm{p}$ & $\mathrm{B}[\%]$ \\
\hline $\begin{array}{l}\text { Sprossdurchmesser } \\
\text { am Wurzelhals }\end{array}$ & - Pflanzendichte & $\begin{array}{l}2000 \\
2001 \\
2002\end{array}$ & $\begin{array}{l}-0,78 \\
-0,95 \\
-0,95\end{array}$ & $\begin{array}{l}0,000 \\
0,000 \\
0,000\end{array}$ & $\begin{array}{l}60,9 \\
91,1 \\
91,1\end{array}$ \\
\hline Anzahl Blätter & - Pflanzendichte & $\begin{array}{l}2000 \\
2001 \\
2002\end{array}$ & $\begin{array}{l}-0,74 \\
-0,73 \\
-0,85\end{array}$ & $\begin{array}{l}0,001 \\
0,001 \\
0,002\end{array}$ & $\begin{array}{l}54,8 \\
53,4 \\
72,7\end{array}$ \\
\hline $\begin{array}{l}\text { Anzahl primärer } \\
\text { Seitentriebe }\end{array}$ & - Pflanzendichte & $\begin{array}{l}2000 \\
2001 \\
2002\end{array}$ & $\begin{array}{l}-0,85 \\
-0,91 \\
-0,92\end{array}$ & $\begin{array}{l}0,000 \\
0,000 \\
0,000\end{array}$ & $\begin{array}{l}72,0 \\
83,0 \\
84,4\end{array}$ \\
\hline $\begin{array}{l}\text { Klassen mit unterschied- } \\
\text { licher Seitentrieblänge }\end{array}$ & - Pflanzendichte & $\begin{array}{l}2000 \\
2001 \\
2002\end{array}$ & $\begin{array}{c}-0,80 \\
-0,83 \\
-\end{array}$ & $\begin{array}{l}0,000 \\
0,000\end{array}$ & $\begin{array}{l}63,2 \\
68,5\end{array}$ \\
\hline $\begin{array}{l}\text { Länge primärer } \\
\text { Seitentriebe }\end{array}$ & - Pflanzendichte & $\begin{array}{l}2000 \\
2001 \\
2002\end{array}$ & $\begin{array}{c}- \\
-0,87 \\
-0,84\end{array}$ & $\begin{array}{l}0,000 \\
0,003\end{array}$ & $\begin{array}{l}75,6 \\
70,2\end{array}$ \\
\hline $\begin{array}{l}\text { Durchmesser primärer } \\
\text { Seitentriebe }\end{array}$ & - Pflanzendichte & $\begin{array}{l}2000 \\
2001 \\
2002\end{array}$ & $\begin{array}{c}- \\
-0,92 \\
-0,87\end{array}$ & $\begin{array}{l}0,000 \\
0,002\end{array}$ & $\begin{array}{l}83,7 \\
74,9\end{array}$ \\
\hline Sprosslänge & - Pflanzendichte & $\begin{array}{l}2000 \\
2001 \\
2002\end{array}$ & $\begin{array}{l}-0,59 \\
-0,96 \\
-0,67\end{array}$ & $\begin{array}{l}0,009 \\
0,000 \\
0,033\end{array}$ & $\begin{array}{l}35,3 \\
91,5 \\
45,0\end{array}$ \\
\hline
\end{tabular}


Tab. 23c: Korrelationen zwischen den Wachstumsparametern der Pflanzen und der Pflanzendichte im Mai 2000 und 2001 am Standort Weendelsbreite und im Mai 2002 am Standort Otto-Hahn-Straße

\begin{tabular}{lccccc}
\hline \multicolumn{1}{c}{$\begin{array}{c}\text { Korrelierende } \\
\text { Merkmale }\end{array}$} & Jahr & $\mathrm{r}$ & $\mathrm{p}$ & $\mathrm{B}[\%]$ \\
\hline Sprossdurchmesser & - Pflanzendichte & 2000 & $-0,88$ & 0,000 & 77,2 \\
am Wurzelhals & & 2001 & $-0,93$ & 0,000 & 86,7 \\
& 2002 & $-0,85$ & 0,002 & 72,6 \\
Sprossdurchmesser & - Pflanzendichte & 2000 & $-0,79$ & 0,000 & 61,9 \\
in 50 cm Höhe & & 2001 & $-0,93$ & 0,000 & 85,9 \\
& 2002 & $-0,61$ & 0,057 & 37,7 \\
Anzahl primärer & - Pflanzendichte & 2000 & $-0,81$ & 0,000 & 66,2 \\
Seitentriebe & & 2001 & $-0,95$ & 0,000 & 89,3 \\
& & 2002 & $-0,79$ & 0,007 & 62,2 \\
Anzahl sekundärer & - Pflanzendichte & 2000 & $-0,80$ & 0,000 & 64,5 \\
Seitentriebe & & 2001 & $-0,91$ & 0,000 & 82,4 \\
& & 2002 & $-0,90$ & 0,001 & 81,3 \\
Anzahl & - Pflanzendichte & 2000 & - & & \\
Seitentriebe & 2001 & - & & \\
& & 2002 & $-0,87$ & 0,001 & 76,0 \\
Sprosslänge & - Pflanzendichte & 2000 & $-0,71$ & 0,001 & 51,1 \\
& 2001 & $-0,80$ & 0,000 & 64,2 \\
& & 2002 & $-0,42$ & 0,229 & 17,4 \\
\hline
\end{tabular}

\subsection{Befallsentwicklung von $P$. chrysocephala in Abhängigkeit von Pflanzendichte und -architektur in den Jahren 1999 bis 2001}

\subsubsection{Zuwanderung von $P$. chrysocephala in die Winterrapsfelder}

Im Jahr 1999 begann die Zuwanderung von P. chrysocephala in die Versuchsfläche bereits in der ersten Septemberwoche. Die Fangzahlen nahmen kontinuierlich bis zur dritten Septemberwoche zu und fielen dann stetig ab (Abb. 9). Das Aktivitätsmaximum von 66,8 Individuen/Gelbschale*Woche wurde bei der Leerung am 23.09. festgestellt.

Auch im Jahr 2000 begann die Zuwanderung von P. chrysocephala Anfang September. Die höchste Aktivitätsdichte wurde bei der Leerung am 13.09. mit 120,0 Käfern/Gelbschale*Woche beobachtet (Abb. 9). Danach sanken die Fangzahlen beständig ab.

Im Jahr 2001 setzte die Zuwanderung von P. chrysocephala im Vergleich zu beiden Vorjahren verspätet ein. Bis zum 24. September waren zunächst nur vereinzelte Tiere zu beobachten. Erst in der darauf folgenden Woche kam es zu einer starken Zuwanderung. Am 01.10. wurden im Mittel 108,8 Käfer/Gelbschale*Woche nachgewiesen (Abb. 9). 


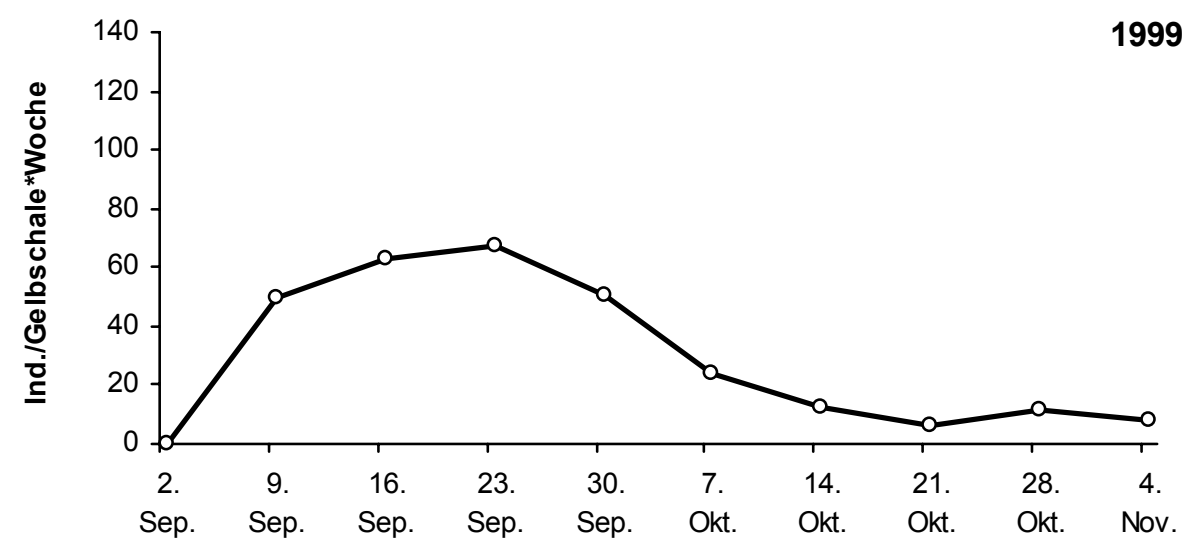

BBCH 12-13 BBCH 14-15 BBCH 16-17
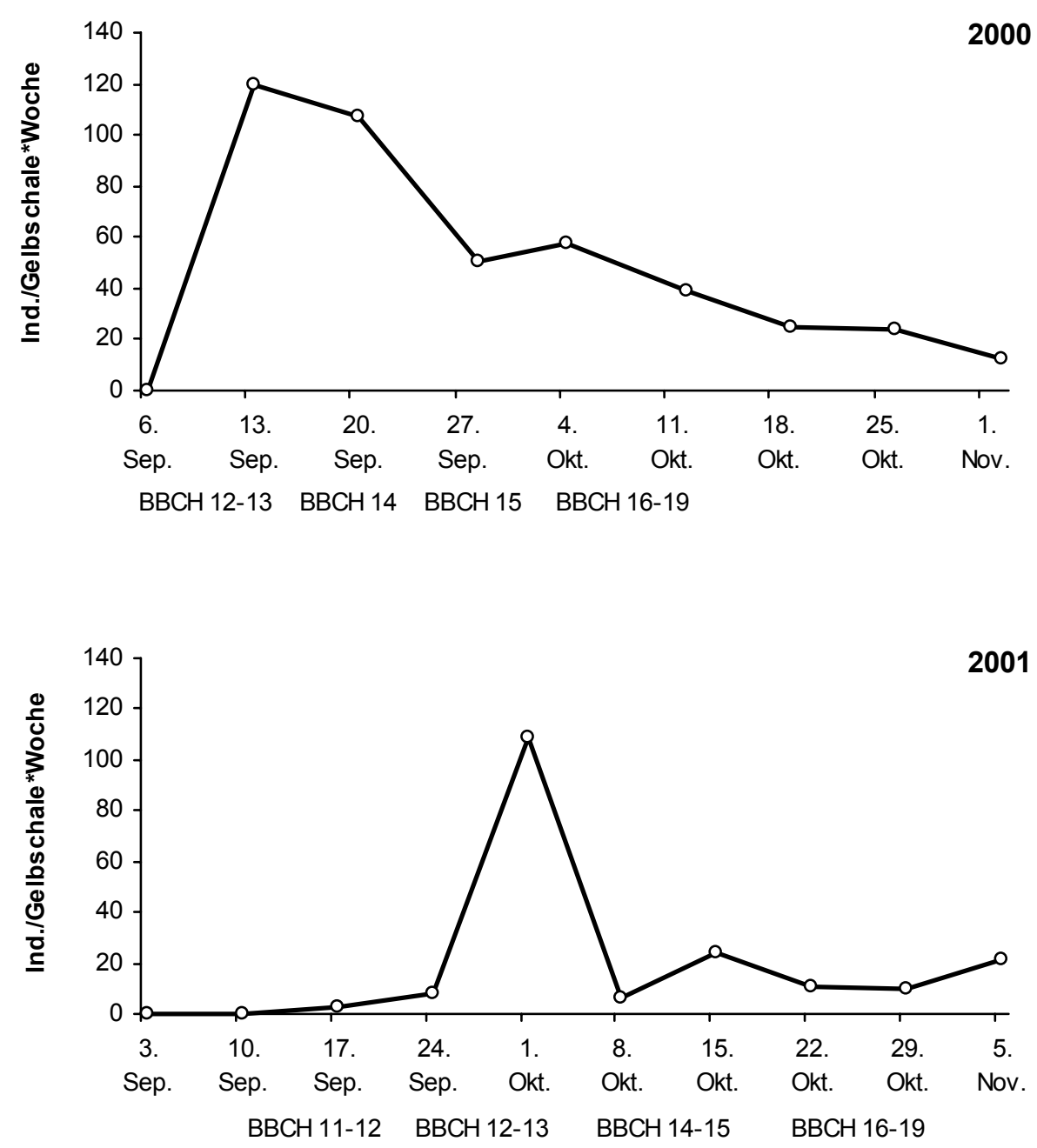

Abb. 9: Mittlerer Gelbschalenfang von $P$. chrysocephala am Standort Weendelsbreite in den Jahren 1999 und 2000 und am Standort Otto-Hahn-Straße im Jahr 2001 


\subsubsection{Besiedlungsdichte und Geschlechterverhältnis der Imagines von $P$. chrysocephala}

Die Imagines von P. chrysocephala traten in den Versuchen 2000 und 2001 in sehr hohen Dichten auf (Tab. 24). Die über drei Erfassungstermine gemittelte Abundanz der Imagines zeigte im Jahr 2000 keine statistisch gesicherten Unterschiede zwischen den Saatstärken, bei der Saatstärke $90 \mathrm{Kö.} / \mathrm{m}^{2}$ allerdings etwas geringere Dichten. Die gemeinsame Verrechnung der Daten aller drei Termine führte zu keinen signifikanten Unterschieden zwischen den Saatstärken (RM-ANOVA, gesamt: $p=0,108, F=2,693$, Weibchen: $p=0,203, F=1,825$, Männchen: $p=0,147, F=2,254)$. Auch an einzelnen Terminen zeigten sich keine Unterschiede in der Besiedlung der Saatstärkevarianten (Abb. 10).

Im Jahr 2001 war die mittlere Abundanz der Imagines von P. chrysocephala bei der Saatstärke $60 \mathrm{Kö.} / \mathrm{m}^{2}$ signifikant höher als bei der Saatstärke $30 \mathrm{Kö.} / \mathrm{m}^{2}$ (Tab. 24). Bei der Betrachtung einzelner Termine ergaben sich nur an den beiden ersten Probeterminen bei der Saatstärke 60 $\mathrm{Kö.} / \mathrm{m}^{2}$ höhere Dichten sowohl für die Individuengesamtzahl als auch für die Abundanz der beiden Geschlechter (Abb. 11). Bei den männlichen Individuen von P. chrysocephala waren die Unterschiede zwischen den beiden Saatstärken am zweiten Probetermin statistisch abzusichern. Die RM-ANOVA ergab einen signifikanten Unterschied zwischen den Saatstärken für die Gesamtzahl der Individuen, nicht jedoch für die einzelnen Geschlechter (RM-ANOVA, gesamt: $p=0,043, F=5,348$, Weibchen: $p=0,072, F=4,045$, Männchen: $p=0,067, F=$ 4,239).

Tab. 24: Mittlere Abundanz ( $\pm \mathrm{SE})$ der Imagines von $P$. chrysocephala über drei Erfassungstermine in Parzellen mit verschiedenen Saatstärken an den Standorten Weendelsbreite und Otto-Hahn-Straße in den Jahren 2000 bzw. 2001

\begin{tabular}{cccccc}
\hline & \multicolumn{5}{c}{ Käfer/m² } \\
Saatstärke & 2000 & & 2001 \\
& $\mathrm{X}_{\mathrm{m}} \pm \mathrm{SE}$ & $\mathrm{X}_{\mathrm{m}} \pm \mathrm{SE}$ & \\
\hline $30 \mathrm{Körner} / \mathrm{m}^{2}$ & $26,2 \pm 2,7$ & a & 29,8 & $\pm 3,0$ & $\mathrm{a}$ \\
$60 \mathrm{Körner} / \mathrm{m}^{2}$ & $24,5 \pm 1,6$ a & $39,9 \pm 3,7$ & $\mathrm{~b}$ \\
$90 \mathrm{Körner} / \mathrm{m}^{2}$ & $19,8 \pm 1,6$ a & - & \\
\hline Unterschiedliche Buchstaben stellen signifikante Unterschiede zwischen den Saatstärken
\end{tabular}

dar, 2000: Kruskal-Wallis-Test, 2001: ANOVA, Tukey-Test, $p<0,05$ 


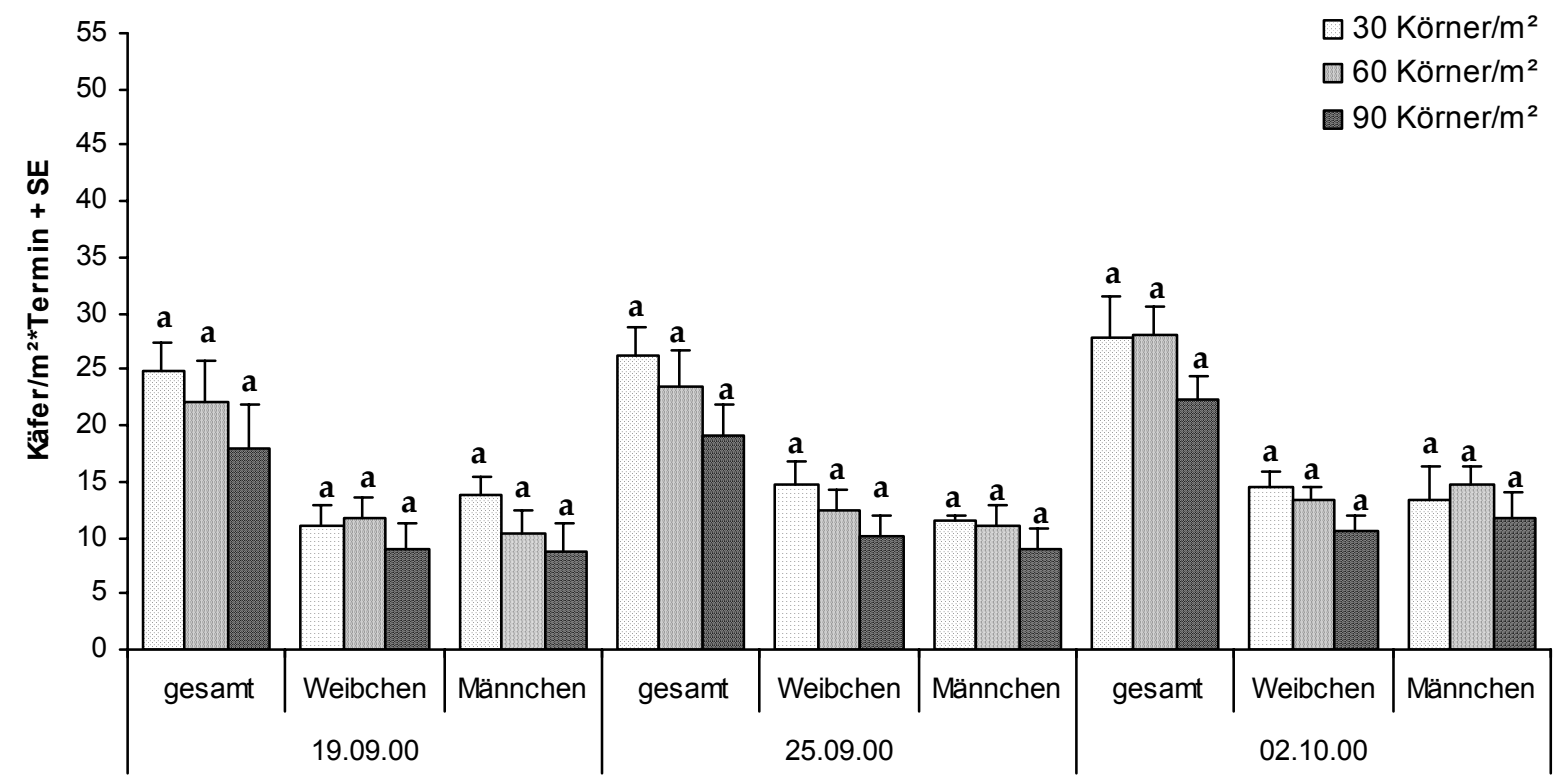

Abb. 10: Mittlere Abundanz der Imagines von P. chrysocephala in Parzellen mit unterschiedlichen Saatstärken am Standort Weendelsbreite im Jahr 2000 (Unterschiedliche Buchstaben stellen signifikante Unterschiede zwischen den Saatstärken dar, Kruskal-Wallis-Test, $\mathrm{p}<0,05$ )

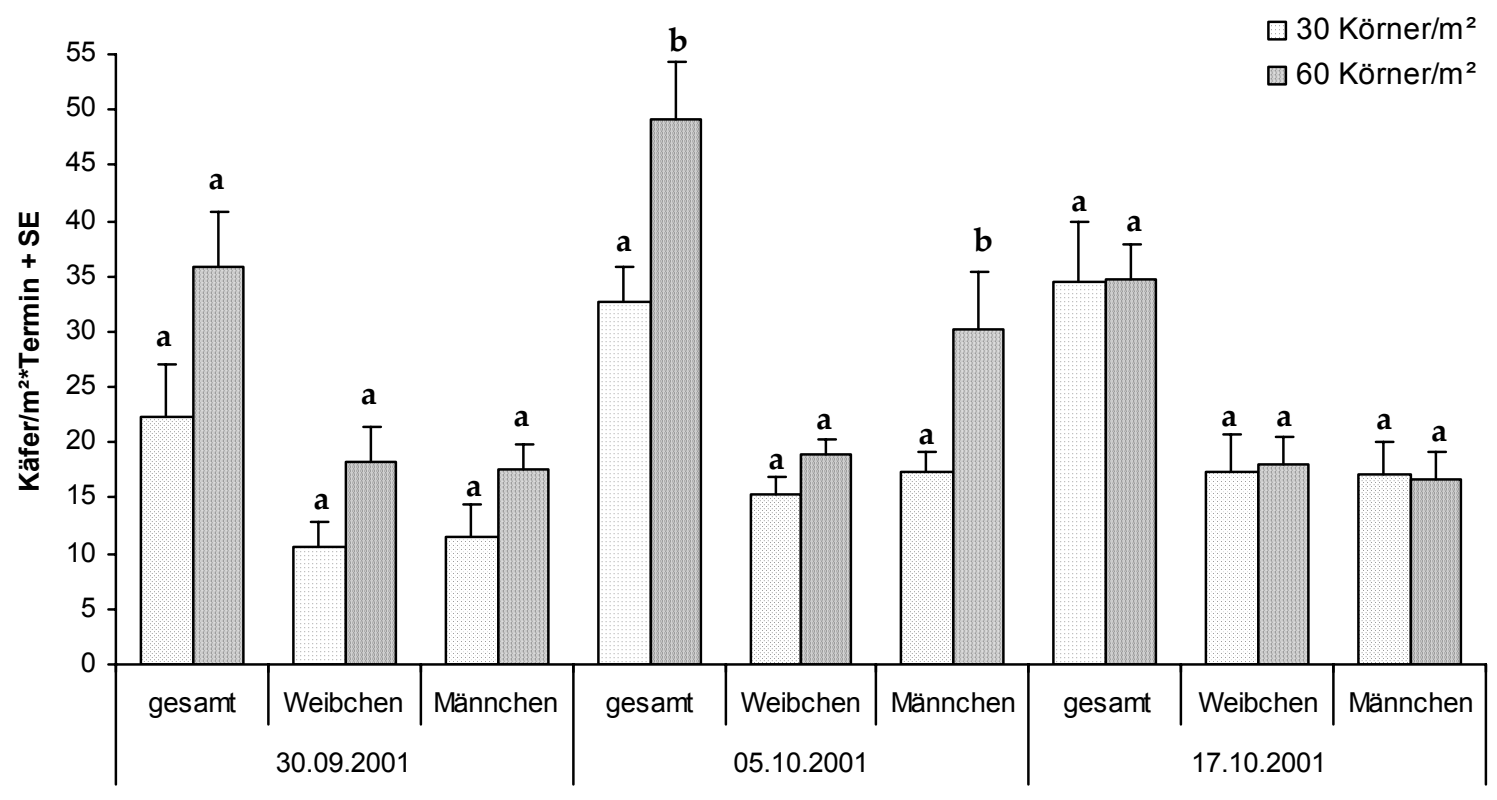

Abb. 11: Mittlere Abundanz der Imagines von P. chrysocephala in Parzellen mit unterschiedlichen Saatstärken am Standort Otto-Hahn-Straße im Jahr 2001 (Unterschiedliche Buchstaben stellen signifikante Unterschiede zwischen den Saatstärken dar, ANOVA, Tukey-Test, $\mathrm{p}<0,05$ ) 
Die Differenzierung der Geschlechter von P. chrysocephala ergab in beiden Jahren bei allen Saatstärken ein nahezu ausgeglichenes Geschlechterverhältnis (Tab. 25). Die mittlere Anzahl der Weibchen betrug im Jahr 2000 in den Saatstärken 30, 60 und 90 Kö./m² 13,3, 12,5 bzw. 10,0 Weibchen $/ \mathrm{m}^{2}$, im Jahr 2001 in den Saatstärken 30 und 60 Kö/m² 14,4 bzw. 18,4 Weibchen $/ \mathrm{m}^{2}$.

Tab. 25: Sexualindex der Imagines von $P$. chrysocephala in Parzellen mit unterschiedlichen Saatstärken an den Standorten Weendelsbreite und Otto-Hahn-Straße in den Jahren 2000 bzw. 2001

\begin{tabular}{ccc}
\hline Saatstärke & \multicolumn{2}{c}{ Sexualindex } \\
& 2000 & 2001 \\
\hline $30 \mathrm{Körner} / \mathrm{m}^{2}$ & 0,51 & 0,49 \\
$60 \mathrm{Körner} / \mathrm{m}^{2}$ & 0,51 & 0,46 \\
$90 \mathrm{Körner} / \mathrm{m}^{2}$ & 0,50 & - \\
\hline
\end{tabular}

\subsubsection{Aktivitätsdichte und Geschlechterverhältnis der Imagines von $P$. chrysocephala}

Bei den in Abbildung 12 dargestellten Aktivitätsverläufen von P. chrysocephala im Versuch 2000 fällt auf, dass in der 2. und 3. Septemberwoche bei der Saatstärke $30 \mathrm{Kö.} / \mathrm{m}^{2} \mathrm{im}$ Vergleich zu den Saatstärken 60 und $90 \mathrm{Kö.} / \mathrm{m}^{2}$ signifikant geringere Aktivitätsdichten zu verzeichnen waren. In der 3. Woche lag nur noch zwischen den Saatstärken 30 und $60 \mathrm{Kö.} / \mathrm{m}^{2}$ ein gesicherter Unterschied vor. Ab dem 4. Oktober konnten keine weiteren Aktivitätsunterschiede mehr festgestellt werden. Die kumulative Aktivitätsdichte (06.09. - 02.11.) war bei den Saatstärken 60 und 90 Kö./m² um den Faktor 1,5 bzw. 1,3 signifikant höher als bei der Saatstärke $30 \mathrm{Kö.} / \mathrm{m}^{2}$ (Abb. 13). Bei den Weibchen von P. chrysocephala konnten statistisch gesicherte Unterschiede zwischen allen drei Saatstärken ermittelt werden. Das Geschlechterverhältnis war bei allen Saatvarianten etwas zugunsten der Männchen verschoben (Tab. 26). 


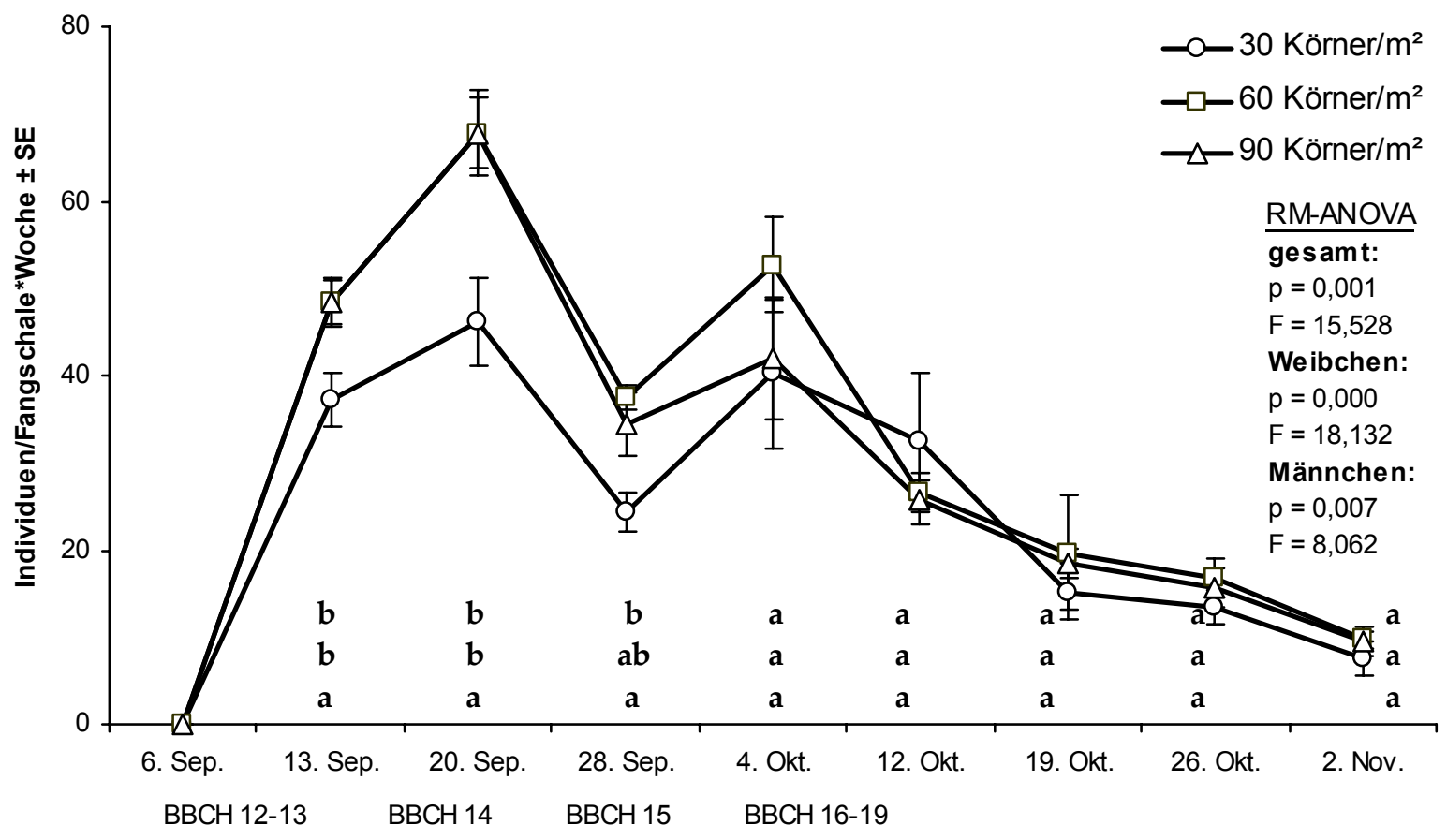

Abb. 12: Mittlere Aktivitätsdichte der Imagines von $P$. chrysocephala in Parzellen mit unterschiedlichen Saatstärken am Standort Weendelsbreite im Jahr 2000 (Unterschiedliche Buchstaben stellen signifikante Unterschiede zwischen den Saatstärken dar, RM-ANOVA, ANOVA, Tukey-Test, $\mathrm{p}<0,05)$

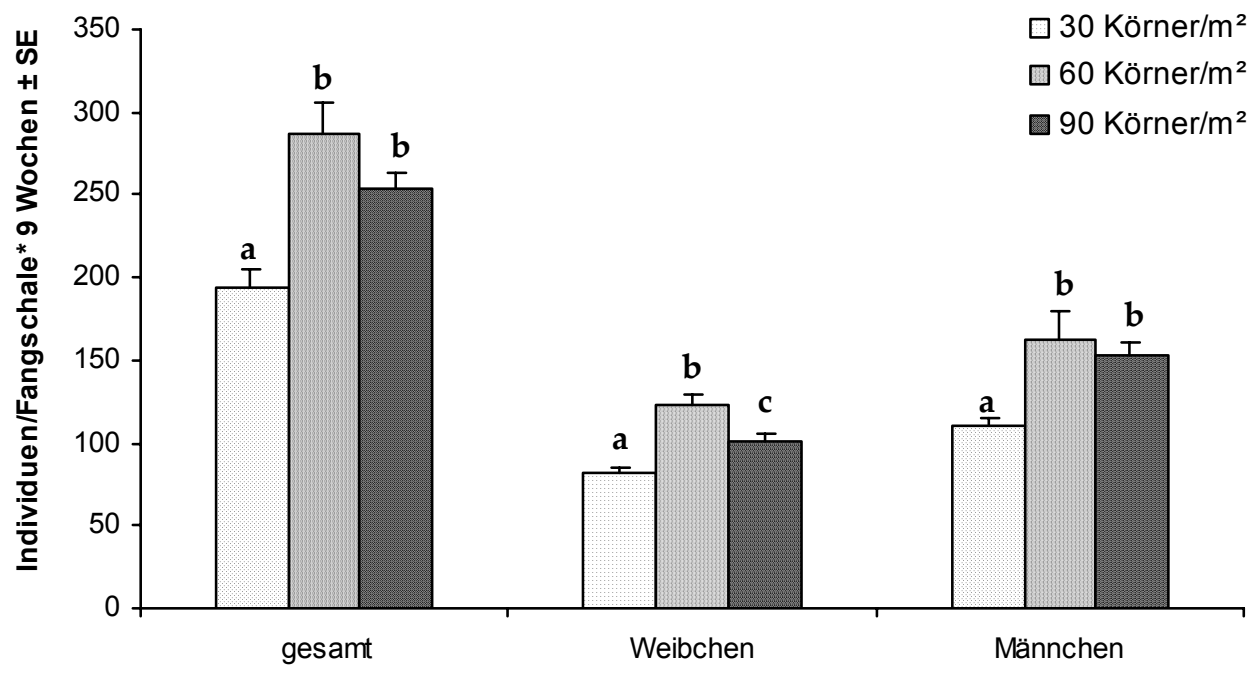

Abb. 13: Mittlere Fangsumme der Imagines von $P$. chrysocephala in Parzellen mit unterschiedlichen Saatstärken am Standort Weendelsbreite im Jahr 2000 (06.09.02.11.) (Unterschiedliche Buchstaben stellen signifikante Unterschiede zwischen den Saatstärken dar, ANOVA, Tukey-Test, $\mathrm{p}<0,05$ ) 
Tab. 26: Sexualindex der Imagines von $P$. chrysocephala in Parzellen mit unterschiedlichen Saatstärken am Standort Weendelsbreite im Jahr 2000 auf Basis der Aktivitätsdichte

\begin{tabular}{cc}
\hline Saatstärke & Sexualindex \\
\hline $30 \mathrm{Körner} / \mathrm{m}^{2}$ & 0,42 \\
$60 \mathrm{Körner} / \mathrm{m}^{2}$ & 0,43 \\
$90 \mathrm{Körner} / \mathrm{m}^{2}$ & 0,40 \\
\hline
\end{tabular}

\subsubsection{Abundanz und Verteilung der Eier von $P$. chrysocephala}

Die Abundanz der Eier von P. chrysocephala im Wurzelbereich der Pflanzen wies im Versuch 2000 bei den unterschiedlichen Saatstärken keine signifikanten Unterschiede auf (Tab. 27). Bei der Saatstärke $30 \mathrm{Kö.} / \mathrm{m}^{2}$ legten die Erdflohweibchen einen Teil der Eier außerhalb des Wurzelbereiches in größerem Abstand von der Pflanze $(>10 \mathrm{~cm}) \mathrm{ab}$.

Bei der genaueren Erfassung der Eidichte in verschiedenen Entfernungsstufen von der Wirtspflanze im Versuch 2001 (0-5 cm, 5-10 cm und 10-15 cm Radius) zeigte sich, dass sowohl die Entfernung von der Pflanze als auch die Saatstärke einen signifikanten Effekt auf die Eiablage hatten: Mit zunehmendem Abstand von der Pflanze ging die Eidichte bei der Saatstärke 30 $\mathrm{Kö.} / \mathrm{m}^{2}$ stärker zurück als bei der Saatstärke $60 \mathrm{Kö} / \mathrm{m}^{2}$ (Tab. 28).

Die Abundanz der Rapserdfloheier pro $\mathrm{m}^{2}$ zeigte im Versuch 2001 bei den Saatstärken 30 und $60 \mathrm{Kö.} / \mathrm{m}^{2}$ mit 66,7 und 99,7 Eiern $/ \mathrm{m}^{2}$ keinen statistisch gesicherten Unterschied, bei der Saatstärke $30 \mathrm{Kö.} / \mathrm{m}^{2}$ allerdings niedrigere Werte (ANOVA, Tukey-Test, p =0,239, F = 1,564) (Abb. 14).

Tab. 27: Mittlere Eidichte $( \pm \mathrm{SE})$ von $P$. chrysocephala innerhalb des Wurzelbereiches der Pflanze und an pflanzenfreien Stellen in Parzellen mit unterschiedlichen Saatstärken am Standort Weendelsbreite im Versuch 2000

\begin{tabular}{|c|c|c|c|c|c|c|c|}
\hline \multirow[t]{3}{*}{ Saatstärke } & \multicolumn{7}{|c|}{ Eier $/ 255 \mathrm{~cm}^{2}$} \\
\hline & \multirow{2}{*}{\multicolumn{3}{|c|}{$\begin{array}{l}\text { Wurzelbereich } \\
X_{m} \pm S E\end{array}$}} & \multicolumn{4}{|c|}{ pflanzenfreie Stellen } \\
\hline & & & & $X_{m}$ & \pm & SE & \\
\hline 30 Körner/m² & 5,2 & $\pm 0,3$ & a $A$ & 1,6 & \pm & 0,2 & $B$ \\
\hline 60 Körner/m² & 5,3 & $\pm 0,8$ & a & & - & & \\
\hline 90 Körner/m² & 3,9 & $\pm 0,6$ & a & & - & & \\
\hline
\end{tabular}


Tab. 28: Mittlere Eidichte ( $\pm \mathrm{SE}$ ) von $P$. chrysocephala in verschiedenen Entfernungsstufen von der Pflanze in Parzellen mit unterschiedlichen Saatstärken am Standort OttoHahn-Straße im Versuch 2001

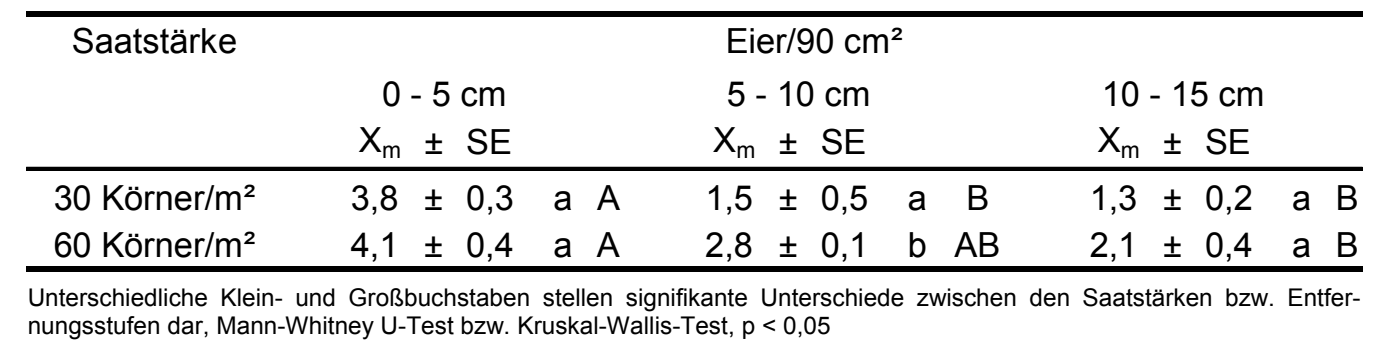

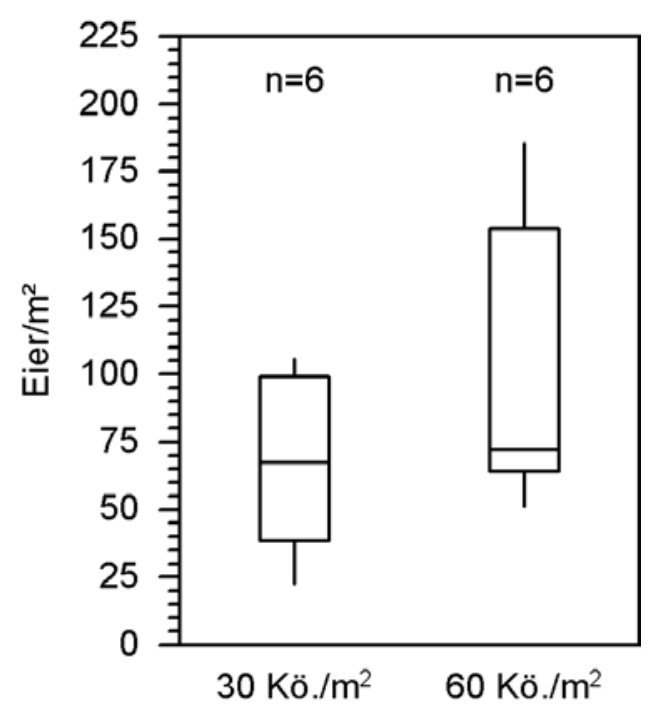

Abb. 14: Mittlere Eidichte von $P$. chrysocephala pro $\mathrm{m}^{2}$ in Parzellen mit unterschiedlichen Saatstärken am Standort Otto-Hahn-Straße im Versuch 2001

\subsubsection{Abundanz der Larven von $\boldsymbol{P}$. chrysocephala}

Die graphische Darstellung der Larvendichten von $P$. chrysocephala in den Versuchen 2000/01 und 2001/02 beschränkt sich im Folgenden jeweils auf den letzten Herbsttermin (16. bzw. 29. November). Im Versuch 1999/00 wurden nur Pflanzenproben vom 11. November untersucht.

Die signifikant höchste Larvendichte/Pflanze wurde in den drei Untersuchungsjahren jeweils in den Parzellen mit der Saatstärke $30 \mathrm{Kö.} / \mathrm{m}^{2}$ ermittelt (Abb. 15). Die Saatstärke $60 \mathrm{Kö.} / \mathrm{m}^{2}$ führte nur im Versuch 1999 nicht zu einer im Vergleich mit $30 \mathrm{Kö} / \mathrm{m}^{2}$ signifikant geringeren Larvendichte. Die Larvenzahl $/ \mathrm{m}^{2}$ wies in allen drei Jahren eine gegenläufige Abstufung zwischen den Saatstärken auf; jedoch ergaben sich lediglich im Versuch 2001 statistisch gesicherte Unterschiede zwischen den Saatstärken 30 und $60 \mathrm{Kö.} / \mathrm{m}^{2}$. Die Larvenzahl war bei der Saat- 
stärke $60 \mathrm{Kö.} / \mathrm{m}^{2}$ mit 466,1 Larven $/ \mathrm{m}^{2}$ signifikant höher als bei der Saatstärke $30 \mathrm{Kö.} / \mathrm{m}^{2}$ mit 335,7 Larven $/ \mathrm{m}^{2}$. Die in Abbildung 15 dargestellten Daten der Larvendichten sind in Tabelle A-1 bis A-3 im Anhang genauer aufgeführt.

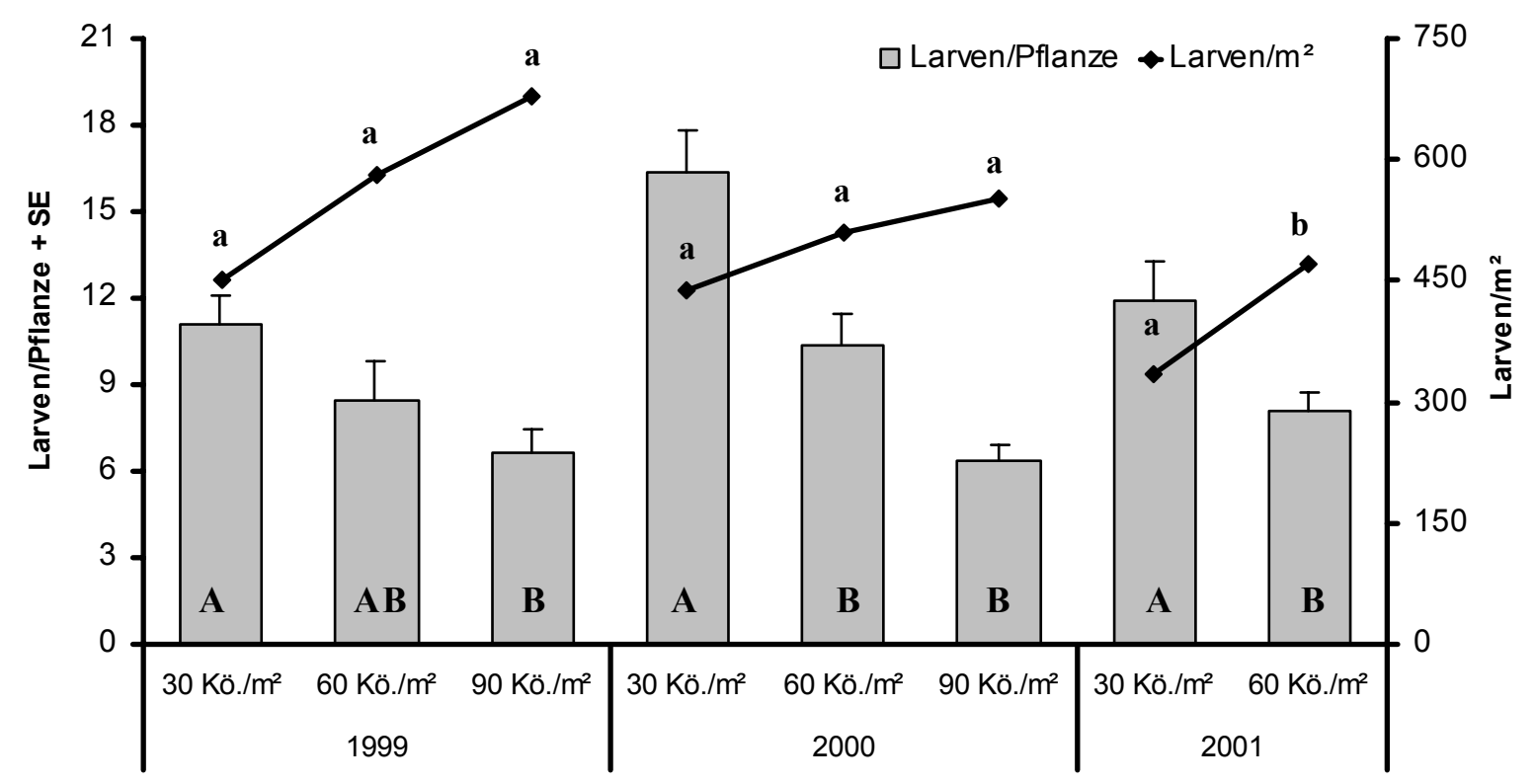

Abb. 15: Mittlere Larvendichte von $P$. chrysocephala in Pflanzen unterschiedlicher Saatstärken im November 1999 und 2000 am Standort Weendelsbreite und im November 2001 am Standort Otto-Hahn-Straße (Verschiedene Buchstaben indizieren signifikante Unterschiede zwischen den Saatstärken im jeweiligen Jahr, ANOVA, TukeyTest, $\mathrm{p}<0,05)$

\subsubsection{Beziehung zwischen der Larvenzahl/Pflanze von $P$. chrysocephala und der Pflan- zendichte}

Zwischen der Abundanz der Larven/Pflanze von P. chrysocephala und der Pflanzendichte zeigte sich in allen drei Versuchsjahren eine signifikante lineare Beziehung (Abb. 16). Mit der Abnahme der Pflanzendichte kam es zu einer Zunahme der Larvenzahl/Pflanze. Die Steigung der Regressionsgeraden verlief im Versuch 1999 flacher $(b=0,07)$ als in den Versuchen 2000 und $2001(\mathrm{~b}=0,16$ bzw. 0,13). In den Versuchen 2000 und 2001 war damit ein stärkerer Anstieg der Larvenzahl/Pflanze mit abnehmender Pflanzendichte festzustellen als im Versuch 1999. Dieser Zusammenhang war im Jahr $2000(B=67$ \%) deutlicher als in den Jahren 1999 und 2001, deren Bestimmtheitsmaße übereinstimmten $(B=39 \%)$. 

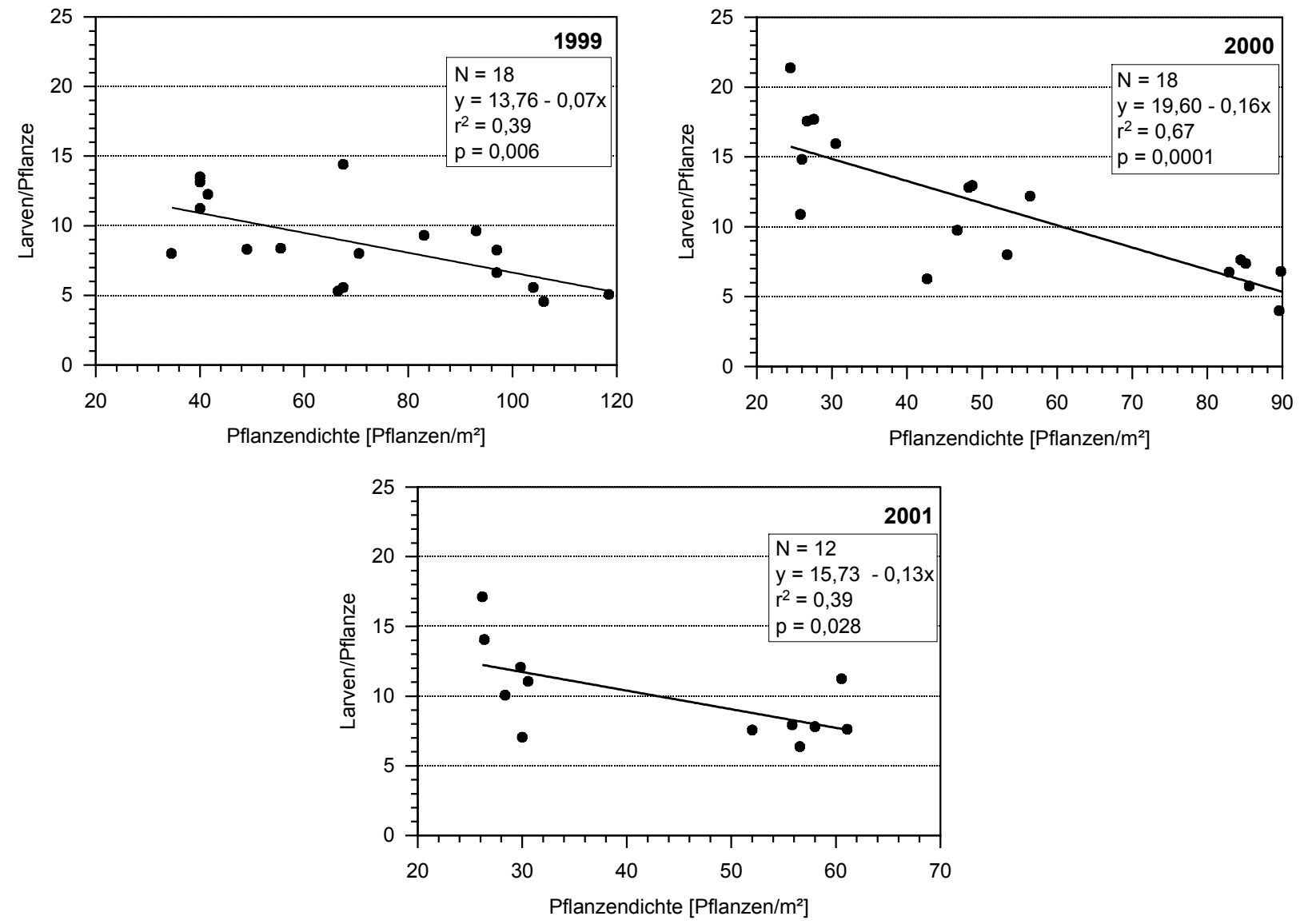

Abb. 16: Abundanz der Larven/Pflanze von P. chrysocephala in Abhängigkeit von Pflanzendichte im November 1999 und 2000 am Standort Weendelsbreite und im November 2001 am Standort Otto-Hahn-Straße

\subsubsection{Beziehung zwischen der Larvenzahl/Pflanze von $\boldsymbol{P}$. chrysocephala und der Pflan- zenarchitektur}

Zwischen der Larvendichte/Pflanze von P. chrysocephala und den Pflanzenparametern Wurzelhalsdurchmesser, Anzahl Blätter und Sprossachsenlänge ergab sich im Herbst 1999 für alle drei Parameter eine signifikante lineare Beziehung (Tab. 29). Im Jahr 2000 konnte nur für die Parameter Wurzelhalsdurchmesser und Anzahl Blätter ein statistisch gesicherter Zusammenhang zur Larvendichte/Pflanze festgestellt werden. Dieser Zusammenhang war bei den beiden Pflanzenparametern mit einem Bestimmtheitsmaß von 79,4 \% bzw. 62,0 \% deutlicher als im Versuch 1999 ( $\mathrm{B}=44,0$ \% bzw. B = 54,1 \%). Im Versuchsjahr 2001 ergab sich für keinen der drei Wachstumsparameter eine signifikante Beziehung zum Larvenbefall/Pflanze. 
Tab. 29: Korrelationen zwischen der Abundanz der Larven/Pflanze von $P$. chrysocephala und den Wachstumsparametern der Pflanzen im November 1999 und 2000 am Standort Weendelsbreite und im November 2001 am Standort Otto-Hahn-Straße

\begin{tabular}{cccccc}
\hline $\begin{array}{c}\text { Korrelierende } \\
\text { Merkmale }\end{array}$ & Jahr & r & p & B [\%] \\
\hline Larven von P. chrysocephala/Pflanze & - Sprossdurchmesser & 1999 & $+0,66$ & 0,007 & 44,0 \\
& am Wurzelhals & 2000 & $+0,89$ & 0,000 & 79,4 \\
& & 2001 & $+0,49$ & 0,100 & 24,4 \\
Larven von P. chrysocephala/Pflanze & - Anzahl Blätter & 1999 & $+0,74$ & 0,001 & 54,1 \\
& & 2000 & $+0,79$ & 0,000 & 62,0 \\
& & 2001 & $+0,54$ & 0,070 & 28,7 \\
Larven von P. chrysocephala/Pflanze & - Sprossachsenlänge & 1999 & $+0,64$ & 0,010 & 41,4 \\
& & 2000 & $+0,40$ & 0,099 & 15,8 \\
& & 2001 & $+0,12$ & 0,701 & 1,6 \\
\hline
\end{tabular}

\subsubsection{Larvendichte von $P$. chrysocephala in Bezug auf die Biomasse der Pflanzen}

Im November 2001 bestand bei der Larvenzahl pro g Biomasse ein signifikanter Unterschied zwischen den Saatstärken 30 und $60 \mathrm{Kö.} / \mathrm{m}^{2}$ : Mit ansteigender Saatstärke nahm die Larvenzahl/g pflanzlicher Biomasse signifikant zu (Tab. 30). Trotz höherer Larvenzahl/Pflanze stand einer Larve in den Pflanzen der Saatstärke $30 \mathrm{Kö.} / \mathrm{m}^{2}$ 1,5-mal mehr Biomasse zur Verfügung als in den Pflanzen der Saatstärke $60 \mathrm{Kö.} / \mathrm{m}^{2}$ (0,54 g bzw. 0,36 g Trockenmasse/Larve).

Im Februar 2001 und Januar 2002 waren zwischen den Larvenzahlen/g Biomasse in den Saatstärkevarianten keine signifikanten Unterschiede zu verzeichnen (Tab. 31). Während im Februar 2001 nur geringfügige Abweichungen zwischen den Saatstärken bestanden, zeigte sich im Januar 2002 eine ähnliche Differenz zwischen den Larvenzahlen/g Biomasse wie im November 2001.

Tab. 30: Mittlere Larvenzahl von $P$. chrysocephala pro Pflanze und pro g pflanzlicher Biomasse ( \pm SE) bei verschiedenen Saatstärken im Herbst 2001 am Standort Weendelsbreite

\begin{tabular}{|c|c|c|c|}
\hline \multirow{3}{*}{ Saatstärke } & \multicolumn{3}{|c|}{ November 2001} \\
\hline & $\begin{array}{l}\text { Larven/ } \\
\text { Pflanze }\end{array}$ & $\begin{array}{c}\text { pflanzliche } \\
\text { Biomasse } \\
\text { [g TrM] }\end{array}$ & $\begin{array}{c}\text { Larven/g } \\
\text { Biomasse }\end{array}$ \\
\hline & $X_{m} \pm S E$ & $\mathrm{X}_{\mathrm{m}} \pm \mathrm{SE}$ & $\mathrm{X}_{\mathrm{m}} \pm \mathrm{SE}$ \\
\hline 30 Körner/m² & $11,9 \pm 1,4 a$ & $6,7 \pm 0,4 a$ & $1,9 \pm 0,3 a$ \\
\hline 60 Körner/m² & $8,1 \pm 0,7 b$ & $2,9 \pm 0,1 b$ & $2,8 \pm 0,2 b$ \\
\hline
\end{tabular}


Tab. 31: Mittlere Larvenzahl von $P$. chrysocephala pro Pflanze und pro g pflanzlicher Biomasse $( \pm$ SE) bei verschiedenen Saatstärken im Winter der Jahre 2001 und 2002 am Standort Weendelsbreite bzw. Otto-Hahn-Straße

\begin{tabular}{|c|c|c|c|c|c|c|}
\hline \multirow{3}{*}{ Saatstärke } & \multicolumn{3}{|c|}{ Februar 2001} & \multicolumn{3}{|c|}{ Januar 2002} \\
\hline & $\begin{array}{l}\text { Larven/ } \\
\text { Pflanze }\end{array}$ & $\begin{array}{c}\text { pflanzliche } \\
\text { Biomasse } \\
\text { [g TrM] }\end{array}$ & $\begin{array}{l}\text { Larven/g } \\
\text { Biomasse }\end{array}$ & $\begin{array}{l}\text { Larven/ } \\
\text { Pflanze }\end{array}$ & $\begin{array}{c}\text { pflanzliche } \\
\text { Biomasse } \\
\text { [g TrM] }\end{array}$ & $\begin{array}{l}\text { Larven/g } \\
\text { Biomasse }\end{array}$ \\
\hline & $X_{m} \pm S E$ & $X_{m} \pm S E$ & $X_{m} \pm S E$ & $X_{m} \pm S E$ & $X_{m} \pm S E$ & $X_{m} \pm S E$ \\
\hline 30 Körner/m² & $18,9 \pm 2,0 a$ & $8,2 \pm 0,8 a$ & $2,4 \pm 0,3 a$ & $15,2 \pm 1,6 a$ & $4,9 \pm 0,3 a$ & $3,2 \pm 0,5 a$ \\
\hline 60 Körner $/ \mathrm{m}^{2}$ & $9,6 \pm 1,3 b$ & $3,9 \pm 0,4 b$ & $2,4 \pm 0,2 a$ & $13,3 \pm 1,5 a$ & $2,8 \pm 0,2 b$ & $4,8 \pm 0,6 a$ \\
\hline 90 Körner/m² & $6,5 \pm 0,5 b$ & $2,2 \pm 0,2 b$ & $3,1 \pm 0,3 a$ & - & - & - \\
\hline
\end{tabular}

Unterschiedliche Buchstaben stellen signifikante Unterschiede zwischen den Saatstärken dar, 2001: ANOVA, Tukey-Test, 2002: Mann-Whitney U-Test, $p<0,05$

Eine deutliche lineare Beziehung zwischen dem Larvenbefall pro Pflanze und dem Trockengewicht der Einzelpflanzen bestand nur im Februar 2001: Die Larvenzahl/Pflanze stieg mit zunehmender Pflanzenmasse signifikant an (Abb. 17).

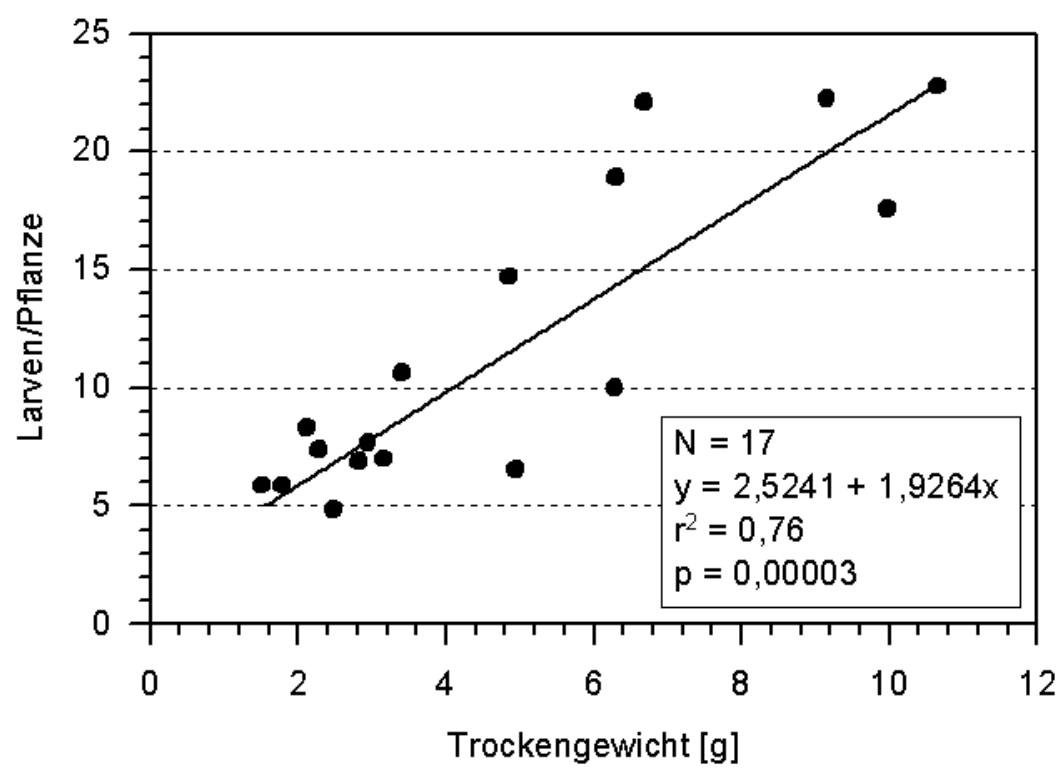

Abb. 17: Mittlere Larvenzahl von $P$. chrysocephala pro Pflanze in Abhängigkeit von der Biomasse der Pflanzen im Februar 2001 am Standort Weendelsbreite 


\subsubsection{Räumliche Verteilung der Eier und Larven von $P$. chrysocephala in den Raps- pflanzen}

\subsubsection{Vertikale Verteilung der Larven von $\boldsymbol{P}$. chrysocephala auf unterschiedliche Blattetagen der Hauptsprossachse}

Bei der Untersuchung des Larvenbefalls einzelner Blattetagen im Herbst 1999 wurden die Larven in den kleinen, nicht geöffneten Blättern der Endknospe gemeinsam erfasst (Abb. 18). Im Herbst 2000 und 2001 hingegen wurden diese Blätter einzeln untersucht und lediglich die jüngsten Blätter der Endknospe (Länge $<5 \mathrm{~mm}$ ) zusammengefasst (Abb. 19 und 20). Bei den Pflanzenuntersuchungen im Februar 2001 und Januar 2002 wurden die jungen Blätter der Endknospe schon ab einer Länge von $10 \mathrm{~mm}$ Länge zusammengefasst, weil die Endknospen bereits deutlich differenzierte Blütenknospenanlagen aufwiesen und bei den kräftigen Pflanzen eine Länge von $5 \mathrm{~mm}$ überschritten wurde. Es wurden nur die Blattetagen der jeweiligen Saatvariante graphisch dargestellt, die bei mindestens $50 \%$ der untersuchten Pflanzen pro Wiederholungsparzelle und mindestens 50 \% der Parzellen der jeweiligen Saatstärke vorhanden waren. Die geöffneten Blätter wurden mit arabischen, die Knospenblätter der Endknospe mit römischen Zahlen bezeichnet.

Im November 1999 entfiel der höchste Larvenbefall bei allen Saatstärken auf die 3. Blattetage; die Larvenzahl sank in den jüngeren Blättern wieder mehr oder weniger stark ab (Abb. 18). Mit Ausnahme der 7. Blattetage trat bei der Saatstärke 90 Kö. $/ \mathrm{m}^{2}$ gegenüber den Saatstärken 30 und $60 \mathrm{Kö.} / \mathrm{m}^{2}$ ein deutlich geringerer Larvenbefall/Blattetage auf. Bemerkenswert ist, dass sich die Larven bei der Saatstärke $30 \mathrm{Kö.} / \mathrm{m}^{2}$ auf mehr Blattetagen verteilen konnten als bei den beiden hohen Saatstärken. Dem relativ hohen Larvenbefall/Pflanze bei der Saatstärke 30 Kö./m² (Tab. A-1, Anhang) stand also eine höhere Anzahl Blätter gegenüber. Bei dem Larvenbefall der Endknospen waren nur marginale Unterschiede zwischen den Saatvarianten erkennbar (vgl. Kap. 3.2.9.2).

In den Versuchen 2000/01 und 2001/02 zeigte sich bei allen Saatvarianten an vier bzw. drei aufeinander folgenden Probeterminen eine stetige Zunahme des Larvenbefalls/Pflanze (Abb. 19 und 20, Tab. A-2 bzw. Tab. A-3, Anhang). Zudem war eine kontinuierliche Umverteilung der Larven in den einzelnen Blattetagen in Richtung Endknospe zu verzeichnen (Abb. 19 und 20), die bei verschiedenen Saatstärken unterschiedlich verlief. 


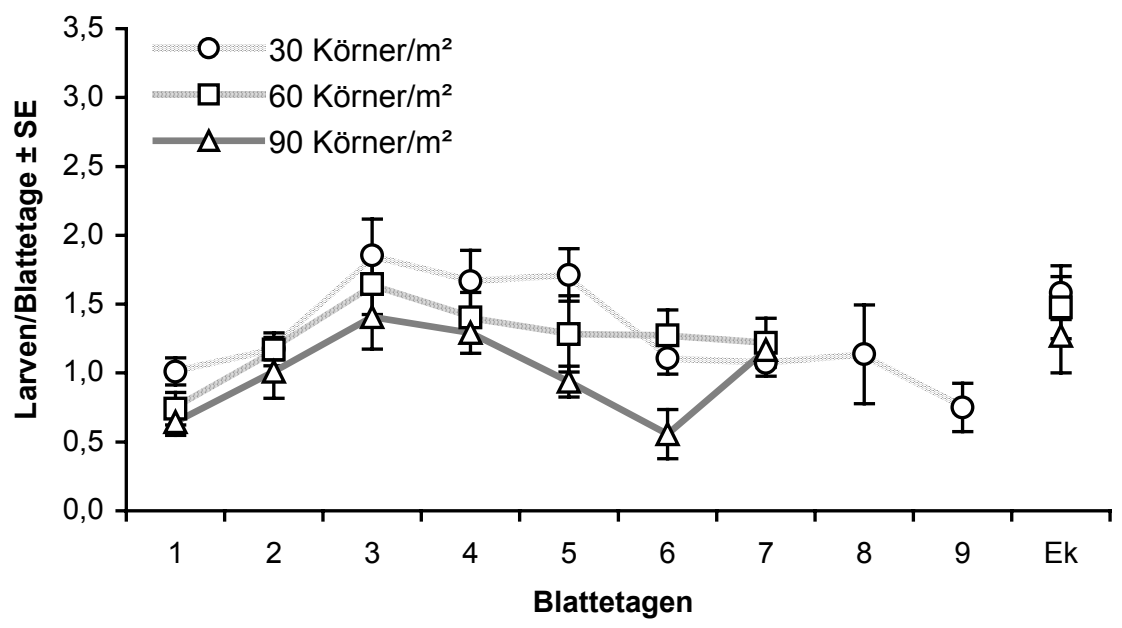

\begin{abstract}
Abb. 18: Vertikale Verteilung der Larven von $P$. chrysocephala in den Blattetagen unterschiedlicher Saatstärken am 11. November 1999 am Standort Weendelsbreite $(E k=$ Knospenblätter der Endknospe)
\end{abstract}

Am 1. Herbsttermin im Versuch 2000/01 wurden alle Blattetagen bei der Saatstärke $90 \mathrm{Kö.} / \mathrm{m}^{2}$ etwa gleichstark befallen (Abb. 19). Am 2. und 3. Termin waren dann hauptsächlich die oberen Blattetagen mit Larven besetzt. Bei der Saatstärke $60 \mathrm{Kö.} / \mathrm{m}^{2}$ ergab sich am 1. Herbsttermin zunächst ebenfalls eine ausgeglichene Befallsverteilung. Am 2. und 3. Termin bot sich ein ähnliches Bild wie bei der Saatstärke $90 \mathrm{Kö.} / \mathrm{m}^{2}$; nur am 2. Termin sank die Larvenzahl ab dem 6. Blatt wieder stark ab. Bei der Saatstärke $30 \mathrm{Kö.} / \mathrm{m}^{2}$ entfiel der höchste Larvenbefall am 1. und 2. Herbsttermin auf die 3. bzw. 4. Blattposition und ging in den jüngeren Blattetagen mehr oder weniger stark zurück. Am 3. Probetermin stieg die Larvenzahl in den einzelnen Blattetagen mit zunehmender Blattetage steil an. Das Befallsmaximum erstreckte sich hierbei einheitlich auf die Blattpositionen 6 bis 10 und fand sich wie auch bei beiden hohen Saatstärken nahe der Endknospe. Beim letzten Untersuchungstermin im Februar 2001 entfiel die höchste Larvenzahl bei der Saatstärke $30 \mathrm{Kö.} / \mathrm{m}^{2}$ auf das 8. und 13. Blatt, bei der Saatstärke 60 Kö./m² auf das 9. Blatt und bei der Saatstärke $90 \mathrm{Kö.} / \mathrm{m}^{2}$ auf die letzten 5 Blätter (Blattpositionen 6 bis 10).

Der maximale Befall in der Endknospe entfiel bei allen Saatstärken an den ersten drei Erfassungsterminen auf die äußeren Knospenblätter. Der Befall sank nach innen deutlich ab. Der Larvenbefall aller Knospenblätter war am 1. Herbsttermin noch gering; bei der Saatstärke 30 Kö. $/ \mathrm{m}^{2}$ war er gegenüber den Saatstärken 60 und $90 \mathrm{Kö.} / \mathrm{m}^{2}$ am geringsten. Mit jedem weiteren Herbsttermin zeichnete sich bei allen Saatstärken eine stetige Zunahme des Larvenbefalls der Knospenblätter ab, bis dann am 16.11.00 das höchste Befallsniveau erreicht wurde. Die Saatstärke $60 \mathrm{Kö.} / \mathrm{m}^{2}$ zeigte hierbei eine deutlich stärkere Befallszunahme als die Saatstärken 
30 und $90 \mathrm{Kö.} / \mathrm{m}^{2}$. Am 15.02.01 war eine auffällige Verringerung des Befalls der Endknospen zu beobachten. Er blieb jedoch bei der Saatstärke $60 \mathrm{Kö.} / \mathrm{m}^{2}$ deutlich höher als bei den Saatstärken 30 und $90 \mathrm{Kö.} / \mathrm{m}^{2}$. Der Larvenbefall der letzten Blattetage innerhalb der Endknospe war bei der Saatstärke $90 \mathrm{Kö.} / \mathrm{m}^{2}$ höher als bei den Saatstärken 30 und $60 \mathrm{Kö.} / \mathrm{m}^{2}$.

Am 1. Herbsttermin im Versuch 2001/02 fand die Besiedlung bei den Saatstärken 30 und 60 $\mathrm{Kö.} / \mathrm{m}^{2}$ vorzugsweise in den unteren Blattetagen statt (Abb. 20). Am 2. Termin zeigte sich bei beiden Saatstärken eine langsame Zunahme des Befalls/Blattetage in Richtung Endknospe. Die höchsten Larvenzahlen konnten bei der Saatstärke $30 \mathrm{Kö.} / \mathrm{m}^{2}$ am viertletzten (Blattposition 8) und bei der Saatstärke $60 \mathrm{Kö.} / \mathrm{m}^{2}$ am vorletzten Blatt (Blattposition 7) festgestellt werden. Am 31. Januar 2002 war bei beiden Saatstärken eine ähnliche Befallsverteilung mit einem Höhepunkt im mittleren Bereich der Blattetagen (30 und $60 \mathrm{Kö.} / \mathrm{m}^{2}$ : Blattposition 7 bzw. 6) zu verzeichnen.

In den Endknospen fanden sich die höchsten Larvenzahlen bei allen Saatstärken, wie im Versuch zuvor, an den beiden Erfassungsterminen im Herbst in den äußeren Knospenblättern und sanken dann nach innen ab. Der zunächst sehr geringe Larvenbefall der Knospenblätter am 1. Herbsttermin stieg stetig an und erreichte am 31. Januar 2002 den höchsten Wert mit einem besonders hohen Befall der letzten Blattetage innerhalb der Endknospe bei der Saatstärke 60 $\mathrm{Kö.} / \mathrm{m}^{2}$ gegenüber der Saatstärke $30 \mathrm{Kö.} / \mathrm{m}^{2}$.

Insgesamt konnte in den beiden Versuchen 2000/01 und 2001/02 festgestellt werden, dass sich die Larven in den schwächer entwickelten Pflanzen der Saatstärken 90 und 60 Kö./m² auf wesentlich weniger Blattetagen verteilen konnten als in den kräftigen Pflanzen der Saatstärke 30 $\mathrm{Kö.} / \mathrm{m}^{2}$. Während der fortschreitenden Umverteilung der Larven in Richtung Endknospe und gleichzeitigem Anstieg des Befallsniveaus/Pflanze erreichten die Larven in den schwächeren Pflanzen früher die letzten entfalteten Blätter nahe der Endknospe als in den kräftigen Pflanzen. Eine Verringerung des Befallsniveaus der Endknospen war bei allen Saatstärken nur im Versuch 2000/01 am letzten Termin zu beobachten. 

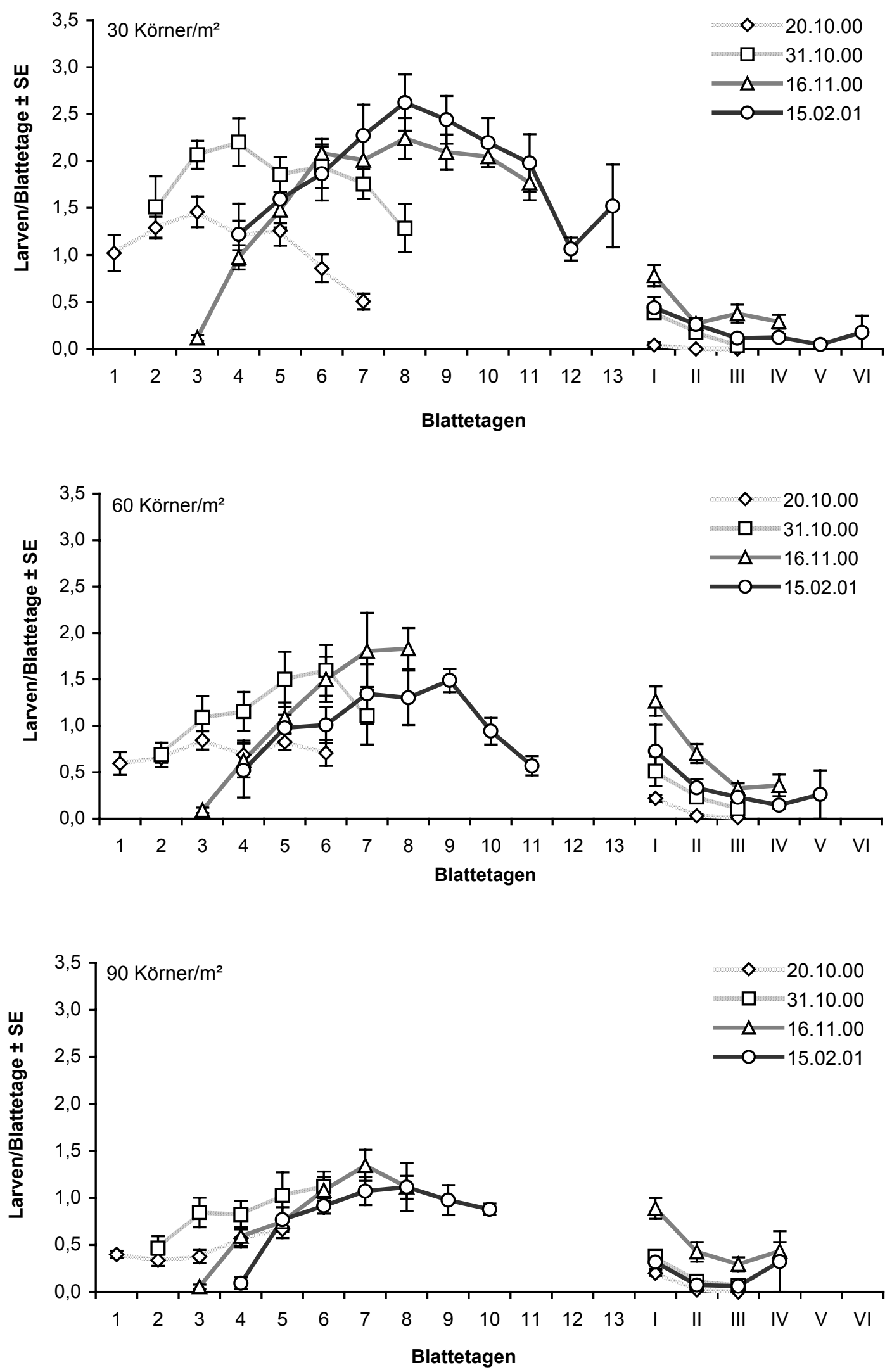

Abb. 19: Vertikale Verteilung der Larven von P. chrysocephala in den Blattetagen unterschiedlicher Saatstärken an vier verschiedenen Terminen im Versuch 2000/01 am Standort Weendelsbreite (Arabische Zahlen bezeichnen voll geöffnete Blätter, römische Zahlen Knospenblätter der Endknospe) 

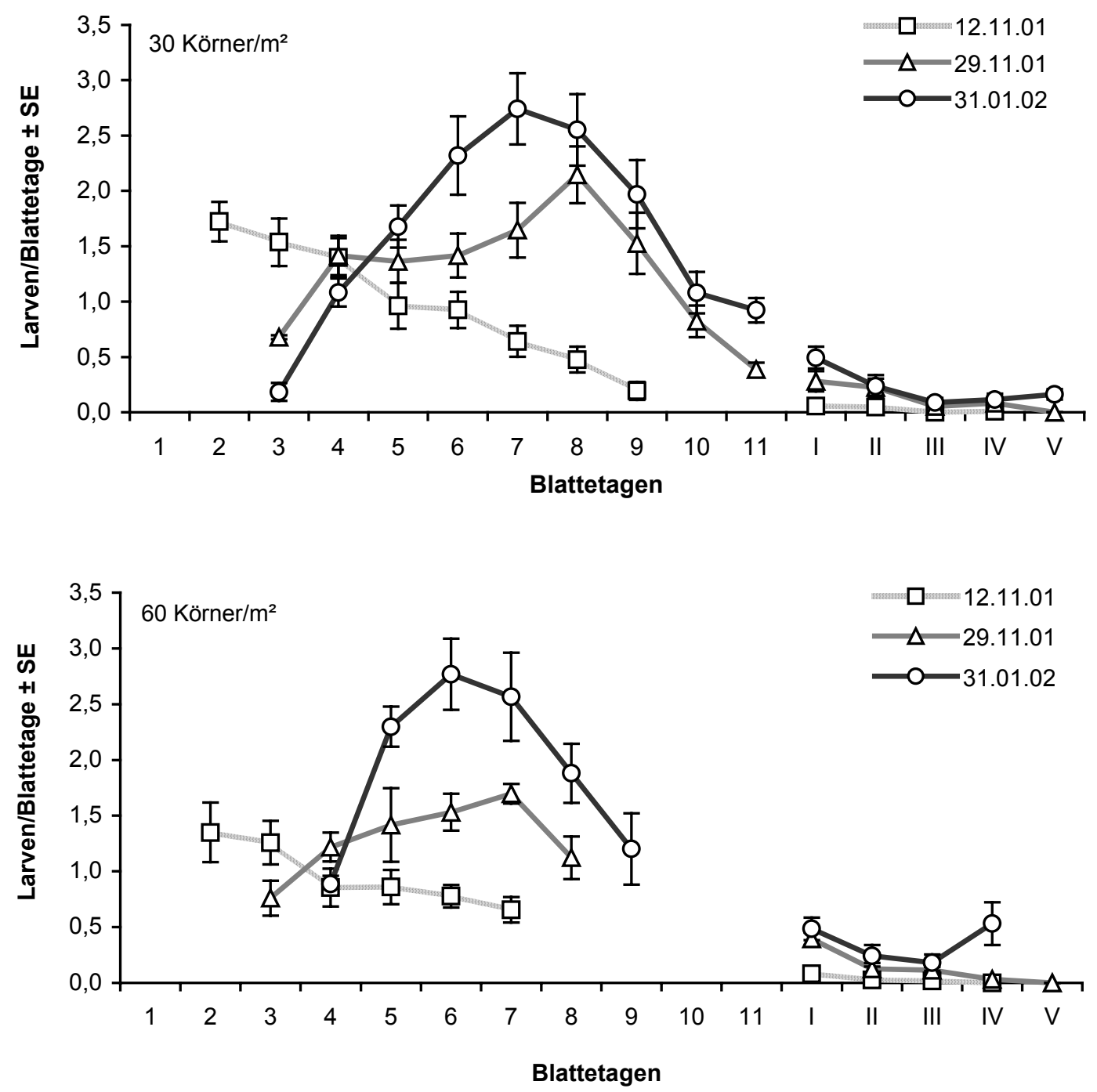

Abb. 20: Vertikale Verteilung der Larven von $P$. chrysocephala in den Blattetagen unterschiedlicher Saatstärken an drei verschiedenen Terminen im Versuch 2001/02 am Standort Otto-Hahn-Straße (Arabische Zahlen bezeichnen voll geöffnete Blätter, römische Zahlen Knospenblätter der Endknospe)

\subsubsection{Verteilung der Larven von $\boldsymbol{P}$. chrysocephala in Endknospe, Seitentriebknospen, Sprossachse und Blättern}

Für die Darstellung der Verteilung der Larven von $P$. chrysocephala in Endknospe, Seitentriebknospen, Sprossachse und Blättern in den Versuchen 1999/00 bis 2001/02 wurden die Daten des jeweils letzten Probentermins im November und des Probentermins im Februar bzw. Januar der Jahre 2001 und 2002 herangezogen. Die vollständigen Larvenzahlen in den Einzelpflanzen im Herbst und Winter sind in den Tabellen A-2 bzw. A-3 im Anhang aufgeführt. 
In allen drei Jahren wurde der überwiegende Anteil der Larven in den Blättern gefunden, während in den Sprossachsen, Seitentrieb- und Endknospen nur ein geringer Teil auftrat. Die absoluten Werte für den Befall der Gesamtpflanzen und der Endknospen sind in Tabelle 32 dargestellt. Die Larvenzahl in der Endknospe wies in den Versuchen 1999 und 2001 nur geringfügige Unterschiede zwischen den Saatstärken auf. Im Versuch 2000 hingegen war die Larvendichte bei der Saatstärke 60 Kö./m² mit 3,0 Larven/Endknospe deutlich höher als bei den Saatstärken 30 und $90 \mathrm{Kö.} / \mathrm{m}^{2}$.

Tab. 32: Mittlere Anzahl Larven von $P$. chrysocephala in der Gesamtpflanze und der Endknospe ( \pm SE) bei verschiedenen Saatstärken im November 1999 und 2000 am Standort Weendelsbreite und im November 2001 am Standort Otto-Hahn-Straße

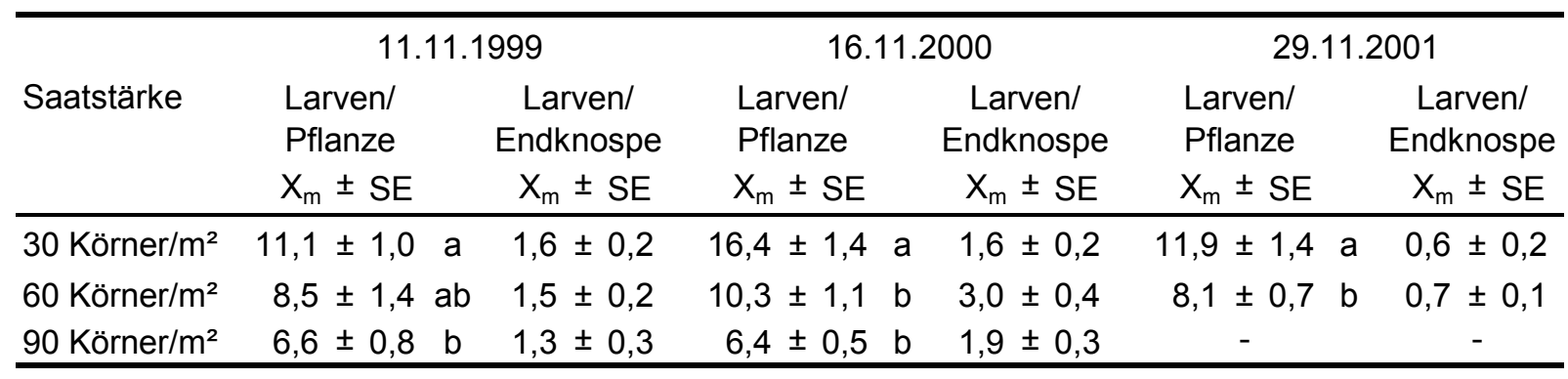

Unterschiedliche Buchstaben indizieren signifikante Unterschiede zwischen den Saatstärken im jeweiligen Jahr, ANOVA, Tukey-Test, $p<0,05$

Der Anteil der Larven in der Endknospe stieg dagegen im Herbst der drei Untersuchungsjahre mit zunehmender Saatstärke an (Abb. 21). Ein signifikanter Unterschied zwischen den Saatstärken zeigte sich jedoch lediglich im Versuch 2000: Der Endknospenbefall war bei den Saatstärken 60 und $90 \mathrm{Kö.} / \mathrm{m}^{2}$ mit 28,7 \% bzw. 28,6 \% an der Gesamtlarvenzahl/Pflanze signifikant höher als bei der Saatstärke $30 \mathrm{Kö.} / \mathrm{m}^{2}$ mit 10,4 \%. Im Vergleich der drei Versuchsjahre ist ersichtlich, dass der relative Befall der Endknospen im Herbst 2001 bei den Saatvarianten 30 und $60 \mathrm{Kö.} / \mathrm{m}^{2}$ mit 4,9 \% bzw. 8,7\% am geringsten war.

Zwischen der Larvenzahl/Gesamtpflanze und der Larvenzahl/Endknospe zeigte sich im Versuchsjahr 1999 ein signifikanter Zusammenhang (Abb. 22): Mit einer Zunahme der Larvenzahl/Pflanze stieg die Larvenzahl/Endknospe an. In den Versuchsjahren 2000 und 2001 konnte keine statistisch gesicherte Beziehung zwischen der Larvendichte/Pflanze und der Larvendichte/Endknospe festgestellt werden. 


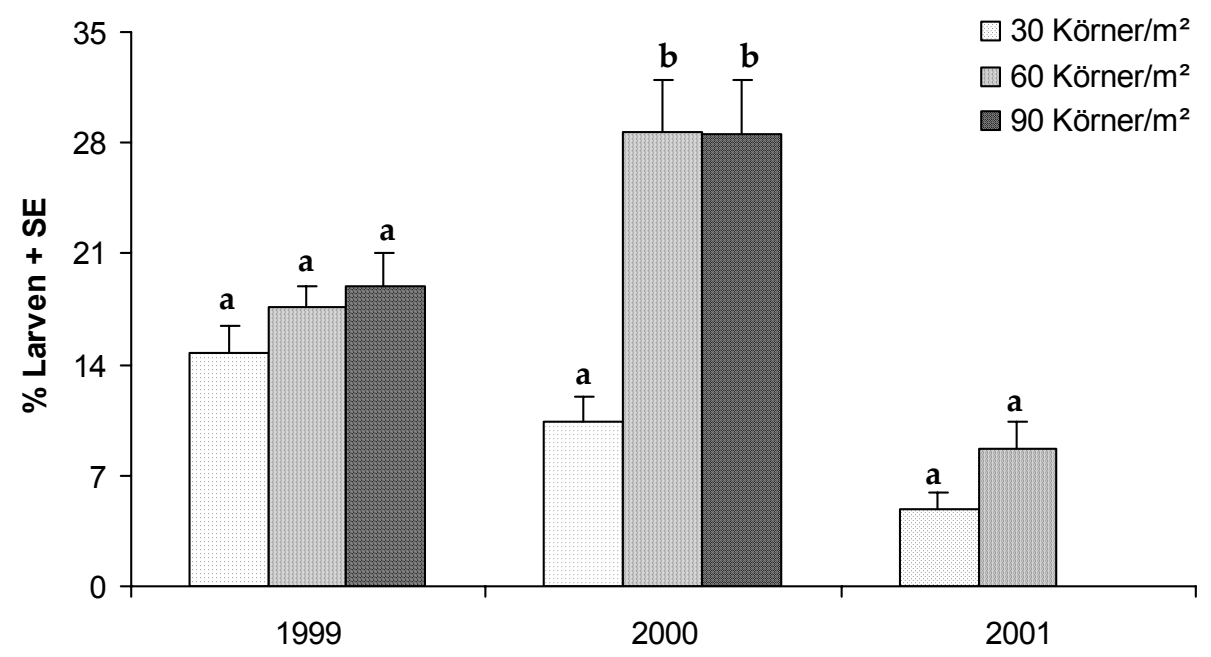

Abb. 21: Mittlerer Anteil Larven von P. chrysocephala in der Endknospe am Gesamtbefall der Pflanze bei verschiedenen Saatstärken im November der Jahre 1999 und 2000 am Standort Weendelsbreite und im November 2001 am Standort Ott-Hahn-Straße (Verschiedene Buchstaben indizieren signifikante Unterschiede zwischen den Saatstärken im jeweiligen Jahr, ARCSIN-Transformation, ANOVA, Tukey-Test, $\mathrm{p}<0,01$ )
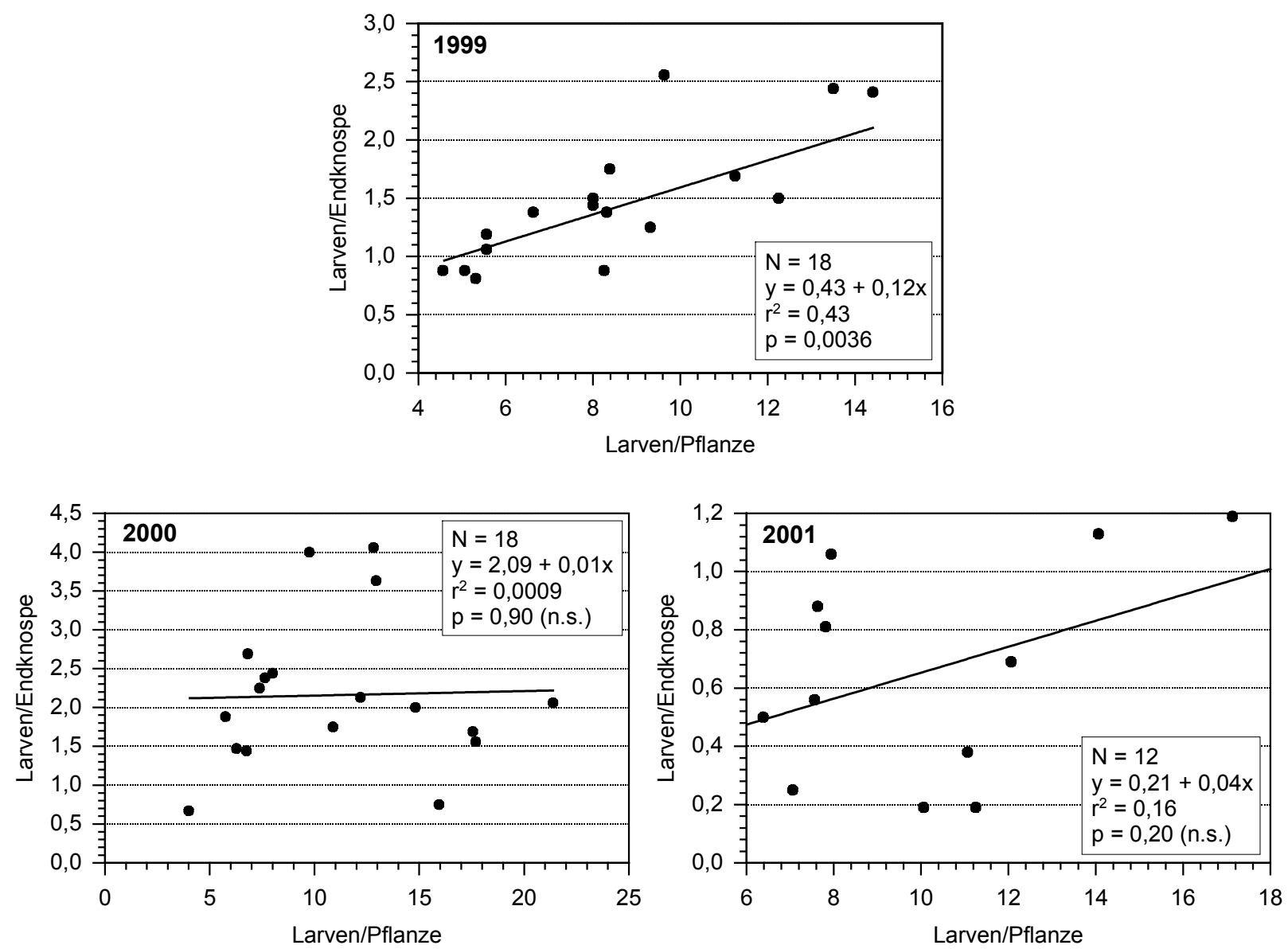

Abb. 22: Mittlere Anzahl Larven von $P$. chrysocephala pro Endknospe in Abhängigkeit vom Einzelpflanzenbefall im November der Jahre 1999 und 2000 am Standort Weendelsbreite und im November 2001 am Standort Otto-Hahn-Straße 
In den Pflanzenproben vom Februar 2001 ergab sich bei allen drei Saatstärken eine Verminderung des relativen Befalls der Endknospen um den Faktor 1,7, 2,4 bzw. 2,3 gegenüber dem November 2000 (vgl. Tab. 33 und Abb. 21). Die Unterschiede zwischen den Saatvarianten waren geringer und konnten, bedingt durch die starke Streuung der Einzelwerte bei der Saatstärke $90 \mathrm{Kö.} / \mathrm{m}^{2}$, nur noch zwischen den Saatstärken 30 und $60 \mathrm{Kö.} / \mathrm{m}^{2}$ statistisch abgesichert werden (Tab. 33). Im Januar 2002 hingegen zeichnete sich bei beiden Saatstärken eine leichte Zunahme des Endknospenbefalls gegenüber dem Herbst 2001 ab (vgl. Abb. 21 und Tab. 34). Der Unterschied zwischen den beiden Saatstärken war nicht statistisch abzusichern.

Die Seitentriebknospen der Rapspflanzen wurden im Herbst der Jahre 1999 und 2000 ebenfalls von P. chrysocephala befallen. Im Versuch 1999 war dies lediglich bei der Saatvariante $30 \mathrm{Kö.} / \mathrm{m}^{2}$ zu verzeichnen, der Befall betrug 1,0 \% der Gesamtlarvenzahl. Im Versuch 2000 wiesen alle Saatstärken Larvenbefall in den Seitentriebknospen auf. Der relative Befall belief sich bei den Saatvarianten 30, 60 und $90 \mathrm{Kö.} / \mathrm{m}^{2}$ auf 1,5 \%, 0,3 \% bzw. 0,1 \%. Die Saatstärke $30 \mathrm{Kö.} / \mathrm{m}^{2}$ unterschied sich hierbei hoch signifikant von den beiden höheren Saatstärken 60 und $90 \mathrm{Kö.} / \mathrm{m}^{2}$, die keine statistisch gesicherten Unterschiede voneinander aufwiesen (ARCSIN-Transformation, ANOVA, Tukey-Test, $\mathrm{p}<0,01)$.

Im Februar 2001 war bei den drei Saatstärken 30, 60 und 90 Kö. $/ \mathrm{m}^{2}$ ein Anstieg des relativen Befalls der Seitentriebknospen um den Faktor 11, 35 bzw. 59 gegenüber dem Herbst zu verzeichnen (Tab. 33). Im darauf folgenden Versuchsjahr 2001/02 trat der Befall der Seitentriebknospen erst im Winter auf (Tab. 34). Insgesamt verhielt sich der relative Befall der Seitentriebe im Winter der beiden Versuche gegenläufig zum Endknospenbefall. Im Versuch 2001 war er bei der Saatstärke $30 \mathrm{Kö.} / \mathrm{m}^{2}$ um den Faktor 1,6 und 1,9 höher als bei den Saatstärken 60 bzw. 90 Kö./m². Im Versuch 2002 war er um den Faktor 1,7 höher als bei der Saatstärke 60 Kö./m². Signifikante Unterschiede waren lediglich im Versuch 2001 zwischen den Saatvarianten 30 und $90 \mathrm{Kö.} / \mathrm{m}^{2}$ festzustellen.

Die Sprossachsen waren im Herbst noch nicht von Larven befallen. Der relative Larvenbefall der Blätter verhielt sich im Herbst in den drei Versuchen gegenläufig zum Befall der End- und Seitentriebknospen und zeigte lediglich im Versuch 2000 statistisch gesicherte Unterschiede zwischen den Saatstärken. Bei der Saatstärke $30 \mathrm{Kö.} / \mathrm{m}^{2}$ war der Anteil der Larven in den Blättern mit 88,2 \% signifikant höher als bei den Saatstärken 60 und $90 \mathrm{Kö.} / \mathrm{m}^{2}$ mit einem Anteil von 71,0 \% bzw. 71,3 \%. Im Herbst 1999 belief sich der relative Blattbefall bei den Saatstär- 
ken 30, 60 und $90 \mathrm{Kö.} / \mathrm{m}^{2}$ auf 84,3 \%, 82,4 \% bzw. 81,1 \%, im Herbst 2001 bei den Saatstärken 30 und $60 \mathrm{Kö.} / \mathrm{m}^{2}$ auf 95,1 \% bzw. 91,3\%.

Im Winter der beiden Versuche 2001 und 2002 zeigte der relative Larvenbefall der Sprossachsen und der Blätter keine statistisch gesicherten Differenzen zwischen den Saatstärken (Tab. 33 und 34).

Tab. 33: Mittlerer Anteil der Larven von P. chrysocephala in Endknospe, Seitentriebknospen, Sprossachse und Blättern am Gesamtbefall der Pflanze $( \pm \mathrm{SE})$ bei verschiedenen Saatstärken im Februar 2001 am Standort Weendelsbreite

\begin{tabular}{|c|c|c|c|c|c|c|}
\hline \multirow{3}{*}{ Saatstärke } & \multicolumn{6}{|c|}{$\%$ Larven, bezogen auf Gesamtbefall der Pflanze } \\
\hline & Endknospe & $\begin{array}{l}\text { Seitentrieb- } \\
\text { knospen }\end{array}$ & \multicolumn{2}{|l|}{ Sprossachse } & \multicolumn{2}{|l|}{ Blätter } \\
\hline & $\mathrm{X}_{\mathrm{m}} \pm \mathrm{SE}$ & $\mathrm{X}_{\mathrm{m}} \pm \mathrm{SE}$ & $X_{m} \pm S E$ & & $X_{m} \pm S E$ & \\
\hline 30 Körner/m² & $6,0 \pm 1,1$ & $15,6 \pm 1,3 \quad a$ & $1,7 \pm 0,6$ & $\mathrm{a}$ & $76,7 \pm 1,4$ & a \\
\hline 60 Körner/m² & $12,1 \pm 1,7$ & $9,7 \pm 2,0 a b$ & $1,2 \pm 0,3$ & a & $77,0 \pm 2,0$ & a \\
\hline 90 Körner/m² & $12,2 \pm 2,7$ al & $8,3 \pm 1,5 b$ & $2,6 \pm 1,1$ & $\mathrm{a}$ & $76,9 \pm 2,4$ & a \\
\hline
\end{tabular}

Unterschiedliche Buchstaben stellen signifikante Unterschiede zwischen den Saatstärken dar, ARCSIN-Transformation, ANOVA, Tukey-Test, $p<0,05$

Tab. 34: Mittlerer Anteil der Larven von P. chrysocephala in Endknospe, Seitentriebknospen, Sprossachse und Blättern am Gesamtbefall der Pflanze ( \pm SE) bei verschiedenen Saatstärken im Januar 2002 am Standort Otto-Hahn-Straße

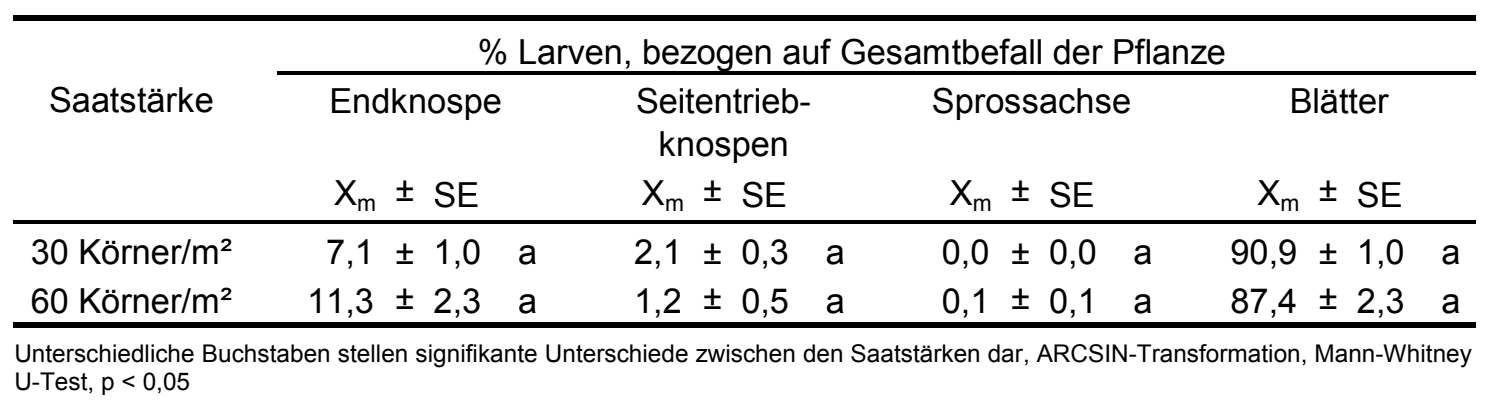




\subsection{Befallsentwicklung von $C$. napi und $C$. pallidactylus in Abhängigkeit von Pflanzen-} dichte und -architektur in den Jahren 2000 bis 2002

\subsubsection{Aktivitätsbeginn von $C$. napi auf den Überwinterungsfeldern}

Im Jahr 2000 wurden die ersten Individuen von C. napi auf den Überwinterungsfeldern am 13.03. in den Gelbschalen gefangen (Abb. 23). Eine stärkere Aktivität war am 20.03., also 7 Tage später, zu verzeichnen. Das Aktivitäts-Maximum wurde am 21.03. mit 73,5 Individuen/Gelbschale ermittelt. Im Jahr 2001 begann der Schlupf von C. napi später als im Vorjahr (Abb. 23). Der erste Fang erfolgte am 30.03., der Höhepunkt der Aktivität trat am 31.03. mit 12,7 Individuen/Gelbschale auf. Das Jahr 2002 zeichnete sich durch einen außerordentlich frühen Schlupfbeginn von C. napi aus (Abb. 23). Die ersten Individuen wurden bereits am 02.02. in den Gelbschalen gefangen. Dann schloss sich eine Phase von etwa 4 Wochen an, in denen keine weiteren Tiere nachzuweisen waren, bevor am 05.03. erneut eine Aktivität zu beobachten war. Der höchste Tagesfang von 17,0 Individuen/Gelbschale wurde am 11.03. erreicht. 

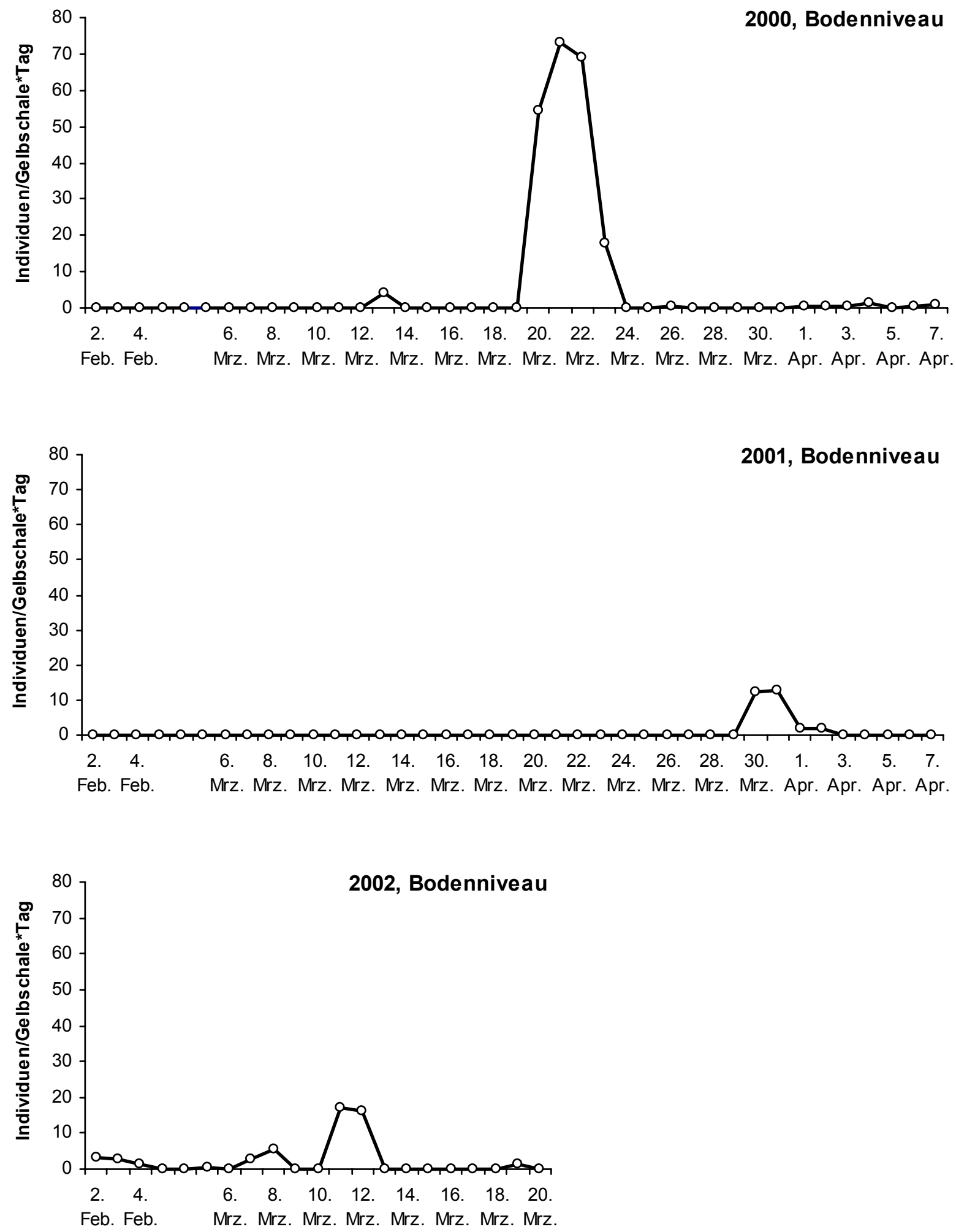

Abb. 23: Aktivitätsverlauf von $C$. napi auf dem Überwinterungsfeld am Standort Weendelsbreite in den Jahren 2000 bis 2002 


\subsubsection{Einwanderung von $C$. napi und $C$. pallidactylus in die Winterrapsfelder}

Der Zuflug von $C$. napi und C. pallidactylus in den Winterrapsbestand wurde am Standort Weendelsbreite in den Versuchsjahren 2000 bis 2001 mit Gelbschalen überwacht, die kontinuierlich der Bestandeshöhe angepasst wurden. Im Jahr 2002 wurden am Standort Otto-HahnStraße neben den Gelbschalen in Bestandeshöhe auch Gelbschalen aufgestellt, die am Boden belassen wurden.

Im Jahr 2000 wiesen die Gelbschalenfänge im Winterraps sowohl für $C$. napi als auch für $C$. pallidactylus den ersten Zuflug am 20.03. aus. Der Hauptzuflug von C. napi wurde am 22.03. mit 4,8 Individuen/Gelbschale*Tag ermittelt (Abb. 24). Der Hauptzuflug von C. pallidactylus wurde am 22.03. und 23.03. mit 6,5 Individuen/Gelbschale erreicht (Abb. 25).

Im Jahr 2001 setzte der Zuflug von C. napi und C. pallidactylus erst Ende März ein. Die ersten Individuen von C. napi wurden am 30.03. in den Gelbschalen gefangen (Abb. 24). Am 31.03. und 02.04. kam es zu einem starken Zuflug von C. napi mit 11,0 bzw. 12,0 Ind./Gelbschale. Das Aktivitäts-Maximum von C. pallidactylus lag am 01.04. und 02.04 . bei 25,8 bzw. 25,5 Käfern/Gelbschale (Abb. 25).

Das Jahr 2002 zeichnete sich durch einen außerordentlich frühen Zuflug von C. napi und C. pallidactylus aus. Die ersten Fänge beider Ceutorhynchus-Arten in den Gelbschalen fanden sich schon am 03.02.. Ein weiterer Zuflug von C. napi war erst am 05.03., 30 Tage später, zu verzeichnen. Der maximale Fang erfolgte am 11.03. mit 15,8 Individuen/Gelbschale in Bestandeshöhe und 25,5 Individuen/Gelbschale in Bodennähe (Abb. 24). Am 11.03. wurden auch die Imagines von $C$. pallidactylus mit 7,3 Tieren/Gelbschale in Bestandeshöhe und 16,3 Tieren/Gelbschale in Bodennähe festgestellt (Abb. 25). Der Hauptfang erfolgte am 12.03. mit 20,3 Käfern/Gelbschale in Bestandeshöhe und 24,8 Käfern/Gelbschale in Bodennähe. Zu einer weiteren hohen Flugaktivität von $C$. pallidactylus kam es am 01.04. mit 10 Individuen/Gelbschale in Bestandeshöhe und 19,5 Individuen/Gelbschale auf Bodenniveau.

Die errechneten Gelbschalen-Fangsummen und die jeweiligen Sexualindices von C. napi und C. pallidactylus im Versuch 2002 zeigten zwischen den beiden Gelbschalenpositionen keine statistisch gesicherten Unterschiede (Tab. 35 und 36). Nur bei C. pallidactylus ergab sich am 01.04. eine auffällige Präferenz der Käfer für die Bodenschalen ( $p=0,070, F=4,857$, ANOVA, LSD-Test) (vgl. Abb. 24 und 25). Der Sexualindex belief sich hierbei auf 0,11. Auch im gesamten Beobachtungsraum war das Geschlechterverhältnis von C. pallidactylus zugunsten männlicher Tiere verschoben (Tab. 36). Der Sexualindex von C. napi war weniger stark zugunsten der Männchen verschoben (Tab. 35). 

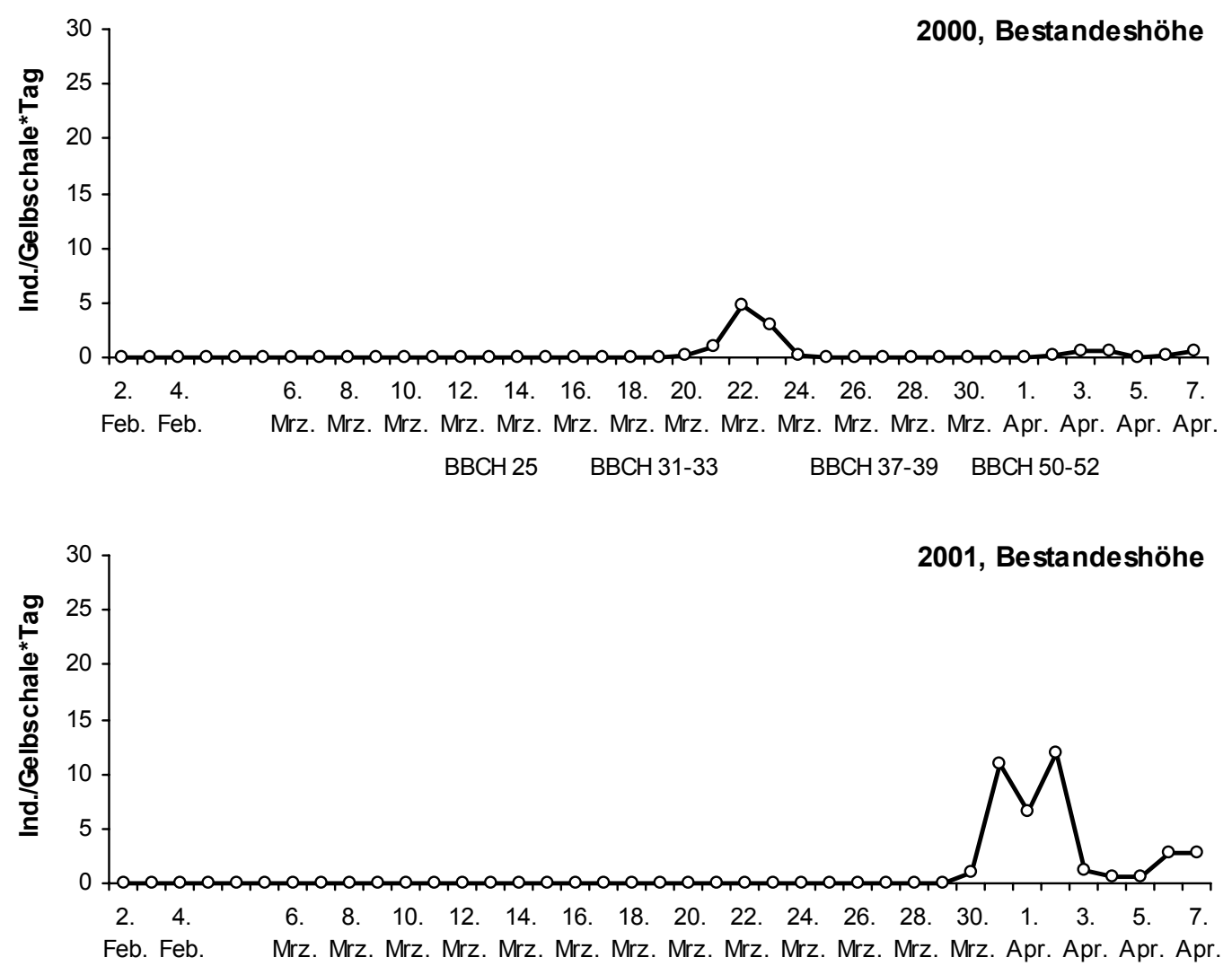

BBCH 25-30 BBCH 33-39 BBCH 50
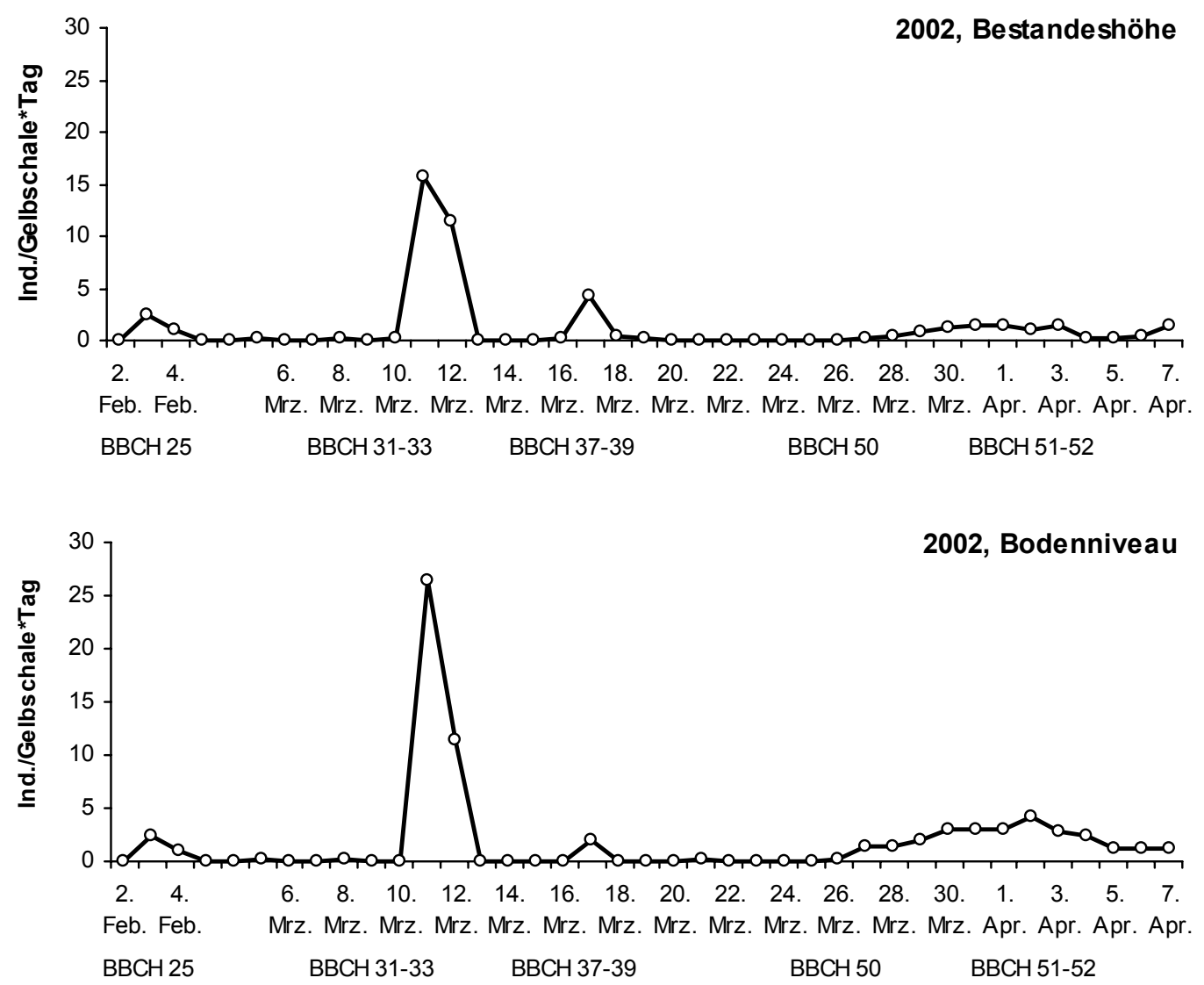

Abb. 24: Gelbschalenfang von C. napi in Bestandeshöhe in den Jahren 2000 bis 2002 und in Bodennähe im Jahr 2002 am Standort Weendelsbreite bzw. Otto-Hahn-Straße 

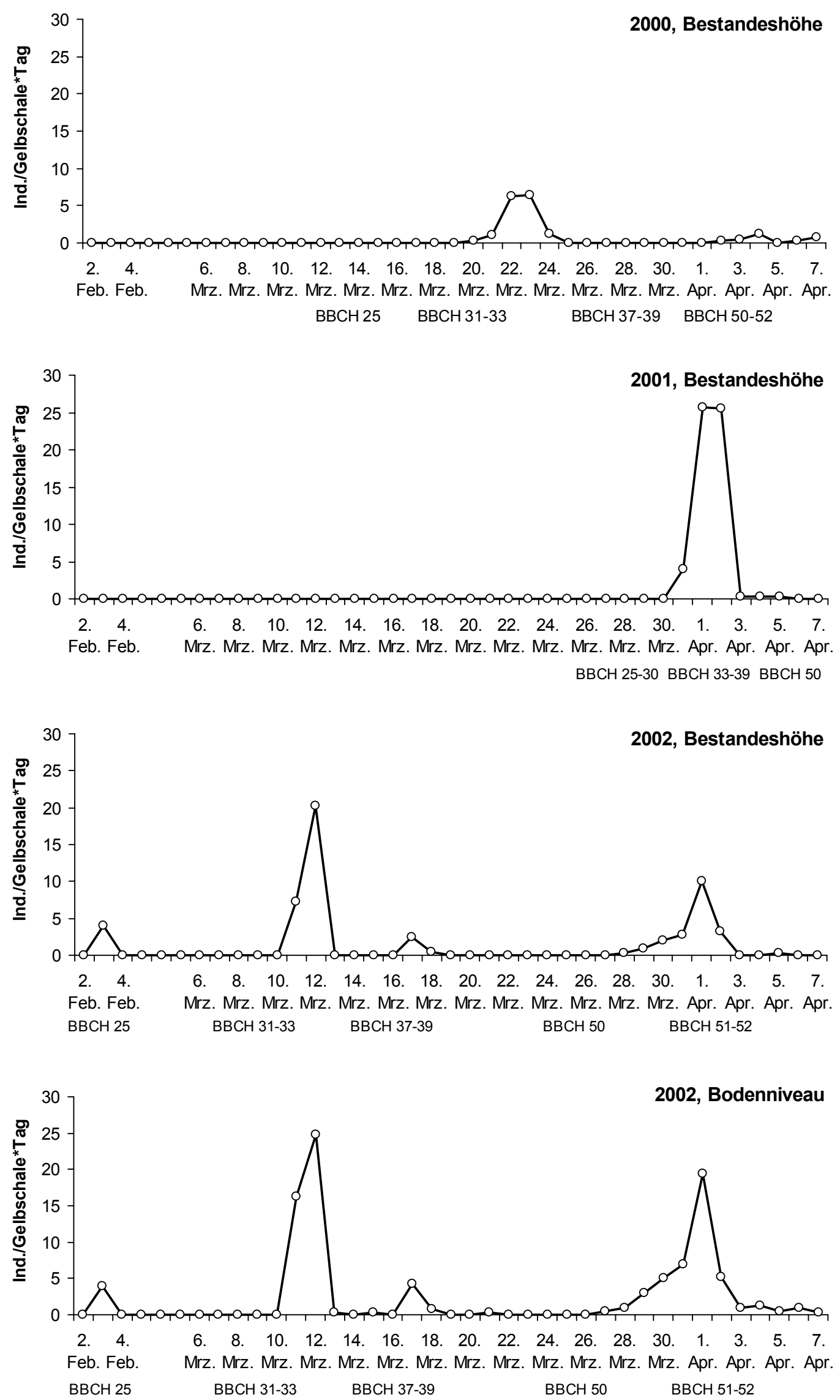

Abb. 25: Gelbschalenfang von C. pallidactylus in Bestandeshöhe in den Jahren 2000 bis 2002 und in Bodennähe im Jahr 2002 am Standort Weendelsbreite bzw. Otto-Hahn-Straße 
Tab. 35: Mittlere Fangsumme und Sexualindex ( $\pm \mathrm{SD}$ ) von $C$. napi in unterschiedlicher Gelbschalenposition am Standort Otto-Hahn-Straße im Jahr 2002 (02.02.-07.04.)

\begin{tabular}{|c|c|c|c|c|}
\hline \multirow{2}{*}{$\begin{array}{c}\text { Gelbschalen- } \\
\text { position }\end{array}$} & \multicolumn{2}{|l|}{ Fangsumme } & \multicolumn{2}{|l|}{ Sexualindex } \\
\hline & $X_{m} \pm S D$ & & $X_{m} \pm S D$ & \\
\hline Bestandeshöhe & $45,5 \pm 16,6$ & a & $0,34 \pm 0,09$ & $a$ \\
\hline Bodenniveau & $70,3 \pm 47,5$ & a & $0,31 \pm 0,15$ & $a$ \\
\hline
\end{tabular}

Tab. 36: Mittlere Fangsumme und Sexualindex ( \pm SD) von C. pallidactylus in unterschiedlicher Gelbschalenposition am Standort Otto-Hahn-Straße im Jahr 2002 (02.02.07.04.)

\begin{tabular}{lcccc}
\hline $\begin{array}{c}\text { Gelbschalen- } \\
\text { position }\end{array}$ & Fangsumme & & Sexualindex & \\
& $\mathrm{X}_{\mathrm{m}} \pm \mathrm{SD}$ & & $\mathrm{X}_{\mathrm{m}} \pm \mathrm{SD}$ & \\
\hline Bestandeshöhe & $52,5 \pm 21,5$ & a & $0,22 \pm 0,03$ & a \\
Bodenniveau & $94,3 \pm 42,0$ & a & $0,18 \pm 0,07$ & a \\
\hline
\end{tabular}

Verschiedene Buchstaben indizieren signifikante Unterschiede zwischen den Saatstärken, ANOVA, LSD-Test, $\mathrm{p}<0,05$

\subsubsection{Abundanz der Eigelege und Larven von $C$. napi und $C$. pallidactylus}

Die Abundanz der Eigelege von C. napi und C. pallidactylus wurde in den Rapspflanzen jeweils im April der drei Untersuchungsjahre erfasst. Die Pflanzen der Saatstärke $30 \mathrm{Kö.} / \mathrm{m}^{2}$ wiesen in allen Jahren einen höheren Befall der Einzelpflanzen als die der Saatstärken 60 und 90 Kö./m² auf (Abb. 26 und 27). Die Befallsunterschiede zwischen den Saatstärken ließen sich bei C. napi nur für das Jahr 2001 und bei C. pallidactylus für die Jahre 2000 und 2002 statistisch absichern. Aufgrund der verspäteten Eiablage von C. pallidactylus im April 2001 wurden nur sehr wenige Eiablagestellen bei der Probenahme erfasst.

Die Larvenzahl/Pflanze von C. napi zeigte im Mai der drei Jahre ähnliche Unterschiede zwischen den Saatstärkevarianten wie die Eizahl/Pflanze im April. Eine Ausnahme bildeten lediglich die Varianten 30 und $60 \mathrm{Kö.} / \mathrm{m}^{2}$ im Jahr 2001, die sich nicht signifikant unterschieden. Bei dem Vergleich der beiden Probetermine wird außerdem deutlich, dass die Larvenzahl/Pflanze gegenüber der Eizahl/Pflanze in allen Untersuchungsjahren zurückging (vgl. Abb. 26 und Abb. 28). Auch bei der Larvenzahl/Pflanze von C. pallidactylus boten sich im Mai der drei Jahre ähnliche Befallsunterschiede zwischen den Saatstärken wie bei der Eizahl/Pflanze im April. Allerdings zeigte sich bei den Larven im Jahr 2001 ein signifikanter Unterschied zwi- 
schen den Saatstärken 30 und 90 Kö./m², im Jahr 2002 dagegen kein signifikanter Unterschied zwischen den Saatstärken 30 und 60 Kö./m² (vgl. Abb. 27 und Abb. 29).

Bezogen auf die Abundanz der Eigelege und Larven $/ \mathrm{m}^{2}$ wies der Befall von C. napi und $C$. pallidactylus keine signifikanten Unterschiede zwischen den Saatstärken auf (Abb. 26, 27, 28 und 29). Bei C. napi zeigten die Ei- und Larvendichten $/ \mathrm{m}^{2}$ jedoch zwischen den Jahren 2000 und 2002 gegenüber dem Jahr 2001 eine gegenläufige Abstufung: In den Versuchen 2000 und 2002 stieg die Befallsdichte $/ \mathrm{m}^{2}$ tendenziell mit zunehmender Saatstärke an, im Versuch 2001 nahm sie mit der Saatstärke ab. Bei C. pallidactylus war lediglich im Versuch 2002 eine graduelle Abstufung zwischen den beiden Versuchssaatstärken 30 und $60 \mathrm{Kö.} / \mathrm{m}^{2}$ zu erkennen, wobei die geringe Saatstärke höhere Befallsdichten $/ \mathrm{m}^{2}$ aufwies. In den Jahren 2000 und 2001 ergab sich bei der Saatstärke $60 \mathrm{Kö.} / \mathrm{m}^{2}$ ein tendenziell höherer Larvenbefall $/ \mathrm{m}^{2}$ als bei den beiden anderen Saatstärken.

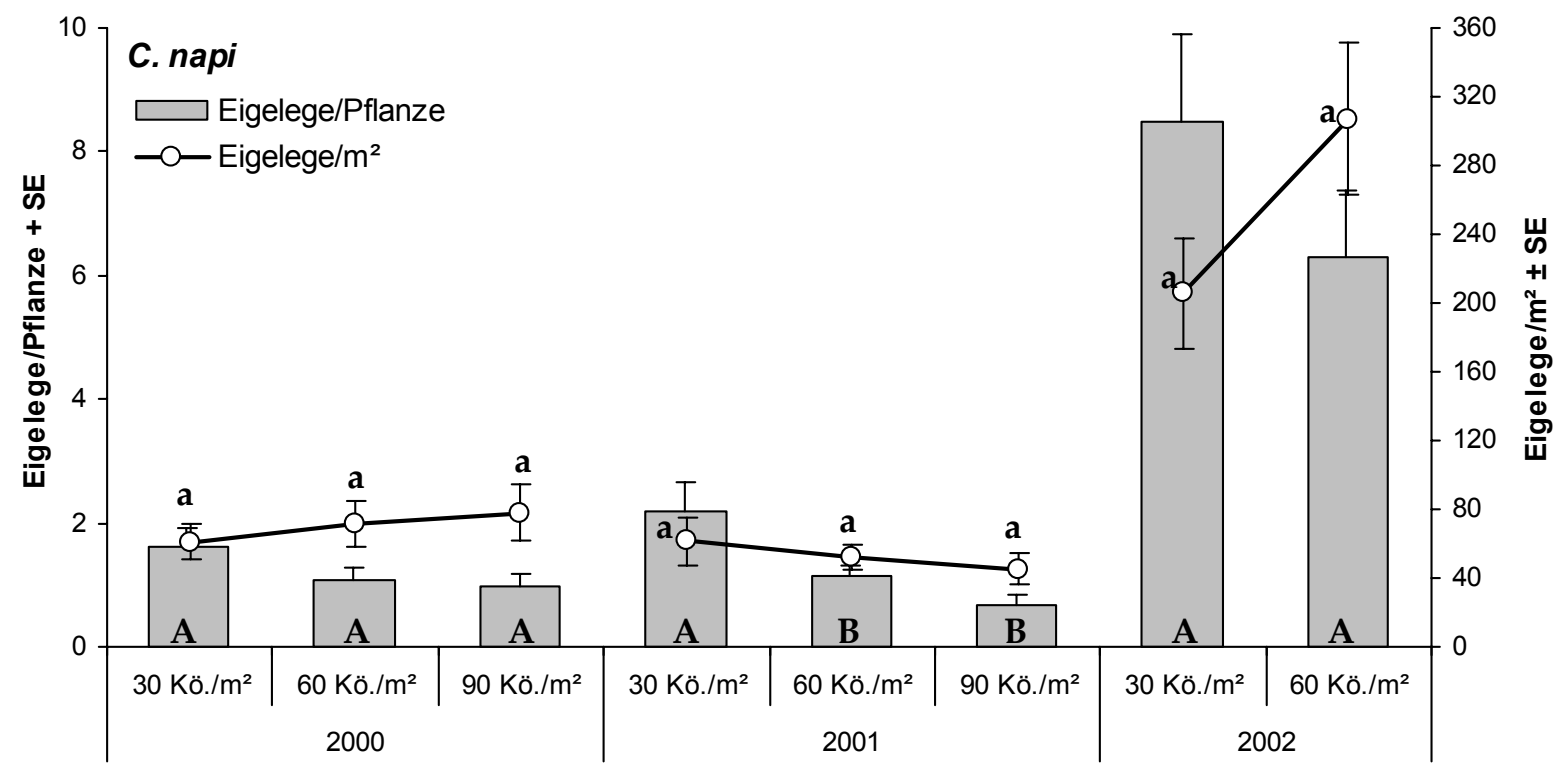

Abb. 26: Mittlere Dichte der Eigelege von C. napi in Pflanzen verschiedener Saatstärken im April 2000 und 2001 am Standort Weendelsbreite und im April 2002 am Standort Otto-Hahn-Straße (Unterschiedliche Buchstaben indizieren signifikante Unterschiede zwischen den Saatstärken im jeweiligen Jahr, 2000 und 2001: ANOVA, TukeyTest, 2002: Mann-Whitney U-Test, $\mathrm{p}<0,05$ ) 


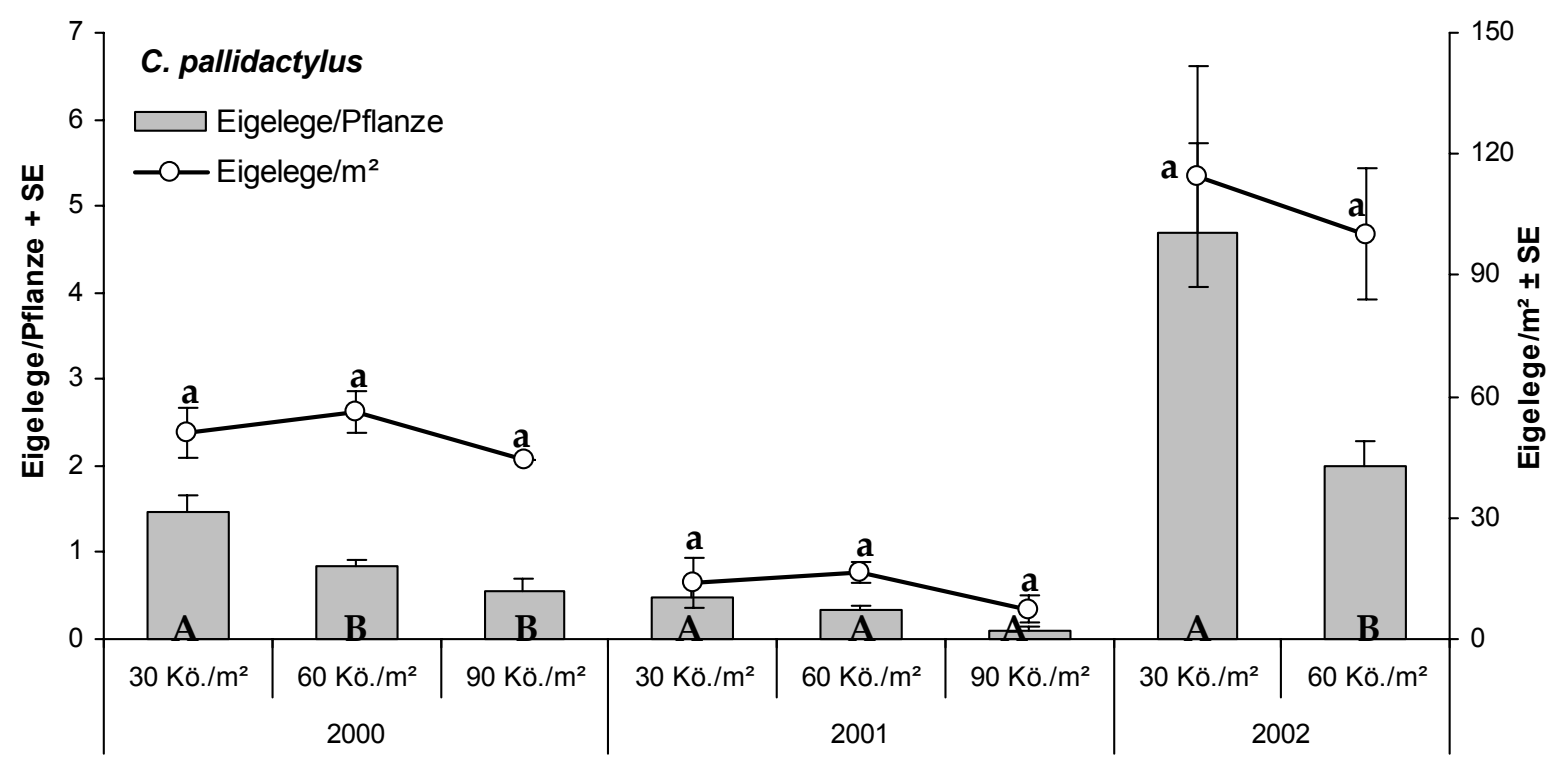

Abb. 27: Mittlere Dichte der Eigelege von C. pallidactylus in Pflanzen verschiedener Saatstärken im April 2000 und 2001 am Standort Weendelsbreite und im April 2002 am Standort Otto-Hahn-Straße (Verschiedene Buchstaben indizieren signifikante Unterschiede zwischen den Saatstärken im jeweiligen Jahr, 2000 und 2001: ANOVA, Tukey-Test, 2002: Mann-Whitney U-Test, $\mathrm{p}<0,05)$

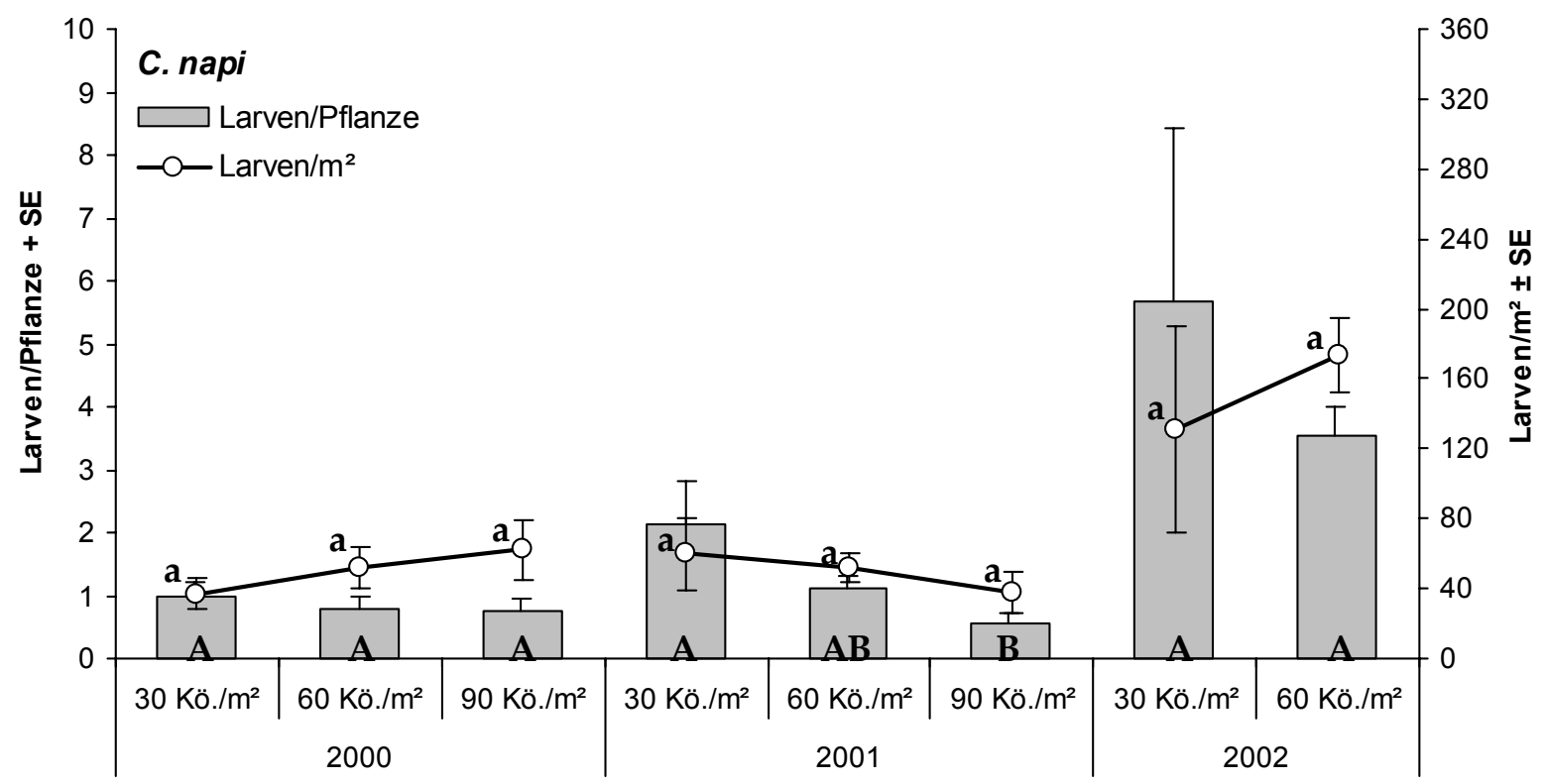

Abb. 28: Mittlere Larvendichte von $C$. napi in Pflanzen verschiedener Saatstärken im Mai 2000 und 2001 am Standort Weendelsbreite und im Mai 2002 am Standort OttoHahn-Straße (Unterschiedliche Buchstaben indizieren signifikante Unterschiede zwischen den Saatstärken im jeweiligen Jahr, 2000 und 2001: ANOVA, Tukey-Test, 2002: Mann-Whitney U-Test, $\mathrm{p}<0,05$ ) 


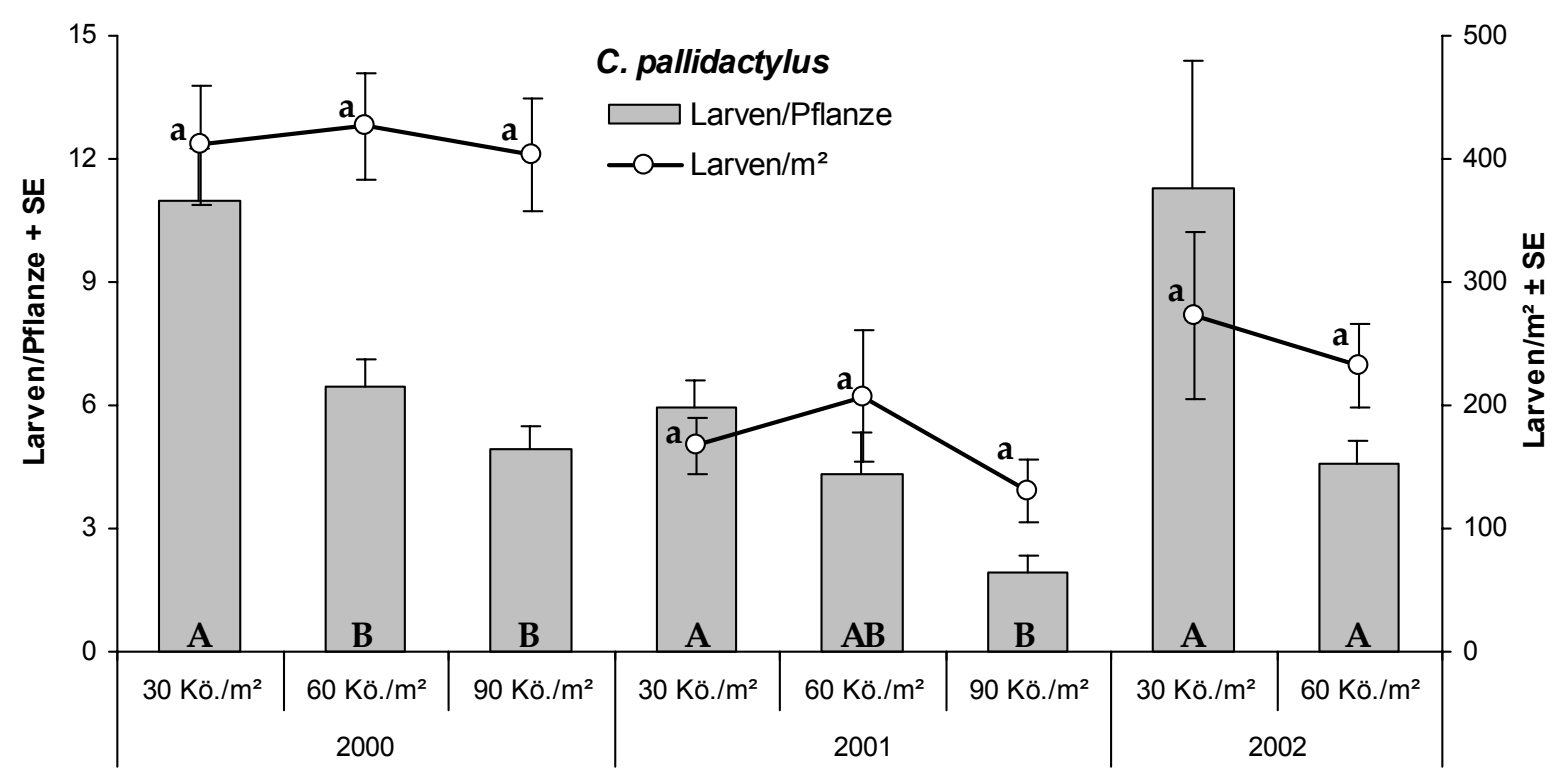

Abb. 29: Mittlere Larvendichte von C. pallidactylus in Pflanzen verschiedener Saatstärken im Mai 2000 und 2001 am Standort Weendelsbreite und im Mai 2002 am Standort Otto-Hahn-Straße (Unterschiedliche Buchstaben stellen signifikante Unterschiede zwischen den Saatstärken im jeweiligen Jahr dar, 2000 und 2001: ANOVA, Tukey-Test, 2002: Mann-Whitney U-Test, $\mathrm{p}<0,05$ )

\subsubsection{Wundkallusbildung der Rapspflanzen als Reaktion auf die Eiablage von $C$. palli- dactylus}

Die Eiablage von $C$. pallidactylus in die Blätter der Rapspflanzen kann zur Bildung von Wundkallusgewebe unmittelbar um das Eigelege führen. Die Eier werden hierbei zerquetscht oder durch das Aufplatzen der Epidermis an die Blattoberfläche geschoben. Dort vertrocknen sie oder fallen heraus. In den Jahren 2000 und 2002 war eine deutliche Reduzierung der Eigelege durch Wundkallusbildung zu beobachten. In beiden Jahren ergaben sich bei der Häufigkeit dieser Reaktion keine signifikanten Unterschiede zwischen den Saatstärken (Tab. 37). Im Jahr 2000 war eine intensivere Wundkallusbildung an den Pflanzen zu beobachten als im Jahr 2002. Darüber hinaus führte die Wundkallusbildung im Versuch $2000 \mathrm{zu}$ einer wesentlich stärkeren Reduzierung der Eigelege als im Versuch 2002. Im April 2001 ließ eine sehr geringe Eiablage von C. pallidactylus keine weitergehende Auswertung zu. 
Tab 37: Häufigkeit der Wundkallusbildung im Bereich der Eigelege von C. pallidactylus in Blättern unterschiedlicher Saatstärken im April der Jahre 2000 und 2002 an den Standorten Weendelsbreite bzw. Otto-Hahn-Straße

\begin{tabular}{|c|c|c|c|c|c|c|c|c|c|c|c|c|c|c|c|c|}
\hline \multirow{3}{*}{ Saatstärke } & \multicolumn{8}{|c|}{2000} & \multicolumn{8}{|c|}{2002} \\
\hline & \multicolumn{4}{|c|}{$\begin{array}{c}\% \text { Eigelege } \\
\text { mit Wundkallus } \\
\text { gesamt }\end{array}$} & \multicolumn{4}{|c|}{$\begin{array}{c}\% \text { Eigelege } \\
\text { mit Wundkallus } \\
\text { ohne Eier }\end{array}$} & \multicolumn{4}{|c|}{$\begin{array}{c}\% \text { Eigelege } \\
\text { mit Wundkallus } \\
\text { gesamt }\end{array}$} & \multicolumn{4}{|c|}{$\begin{array}{c}\% \text { Eigelege } \\
\text { mit Wundkallus } \\
\text { ohne Eier }\end{array}$} \\
\hline & $\mathrm{X}_{\mathrm{m}}$ & \pm & SE & & $X_{m}$ & \pm & SE & & $\mathrm{X}_{\mathrm{m}}$ & \pm & SE & & $\mathrm{X}_{\mathrm{m}}$ & \pm & SE & \\
\hline 30 Körner/m² & 79,8 & \pm & 5,5 & $a$ & 54,6 & \pm & 5,4 & $a$ & 37,2 & \pm & 3,5 & $a$ & 13,4 & \pm & 2,5 & $a$ \\
\hline 60 Körner/m² & 78,0 & \pm & 5,9 & a & 47,6 & \pm & 4,5 & $a$ & 34,3 & \pm & 6,8 & a & 15,6 & \pm & 5,5 & a \\
\hline 90 Körner/m² & 69,8 & \pm & 10,7 & a & 50,4 & \pm & 15,9 & $a$ & & - & & & & - & & \\
\hline
\end{tabular}

Unterschiedliche Buchstaben stellen signifikante Unterschiede zwischen den Saatstärken dar, ARCSIN-Transformation, 2000: ANOVA, Tukey-Test, 2002: Mann-Whitney U-Test, $p<0,05$

\subsubsection{Beziehung zwischen der Anzahl der Eigelege/Pflanze von $C$. napi und der Pflan- zendichte}

Zwischen der Anzahl der Eigelege/Pflanze von C. napi und der Pflanzendichte ergab sich nur im Versuch 2001 eine hoch signifikante lineare Beziehung (Abb. 30). Mit zunehmender Pflanzendichte fiel die Anzahl der Eigelege/Pflanze deutlich ab. Bei separater Betrachtung der Eiablage in die Haupt- und Seitentriebe wurden für beide Eiablageorte signifikante Korrelationen festgestellt, deren Bestimmtheitsmaß mit 38,5 \% bzw. 30,0 \% auf einen stärkeren Zusammenhang zwischen der Eiablage in die Haupttriebe und der Pflanzendichte in diesem Versuch hindeutet (Tab. 38).

Bei den Versuchen 2000 und 2002 ergaben sich keine absicherbaren Beziehungen zwischen der Eiablage in die Haupttriebe und der Pflanzendichte (Tab. 38). Zwischen der Eiablage in die Seitentriebe und der Pflanzendichte war im Versuch 2002 eine hochsignifikante Beziehung $(B=69,5 \%)$ zu verzeichnen. Der Zusammenhang zwischen den beiden Parametern im Versuch 2000 (B = 19,7 \%) war nur auf einem schwächeren Signifikanzniveau bei einer Fehlerwahrscheinlichkeit von $10 \%$ statistisch abzusichern. 

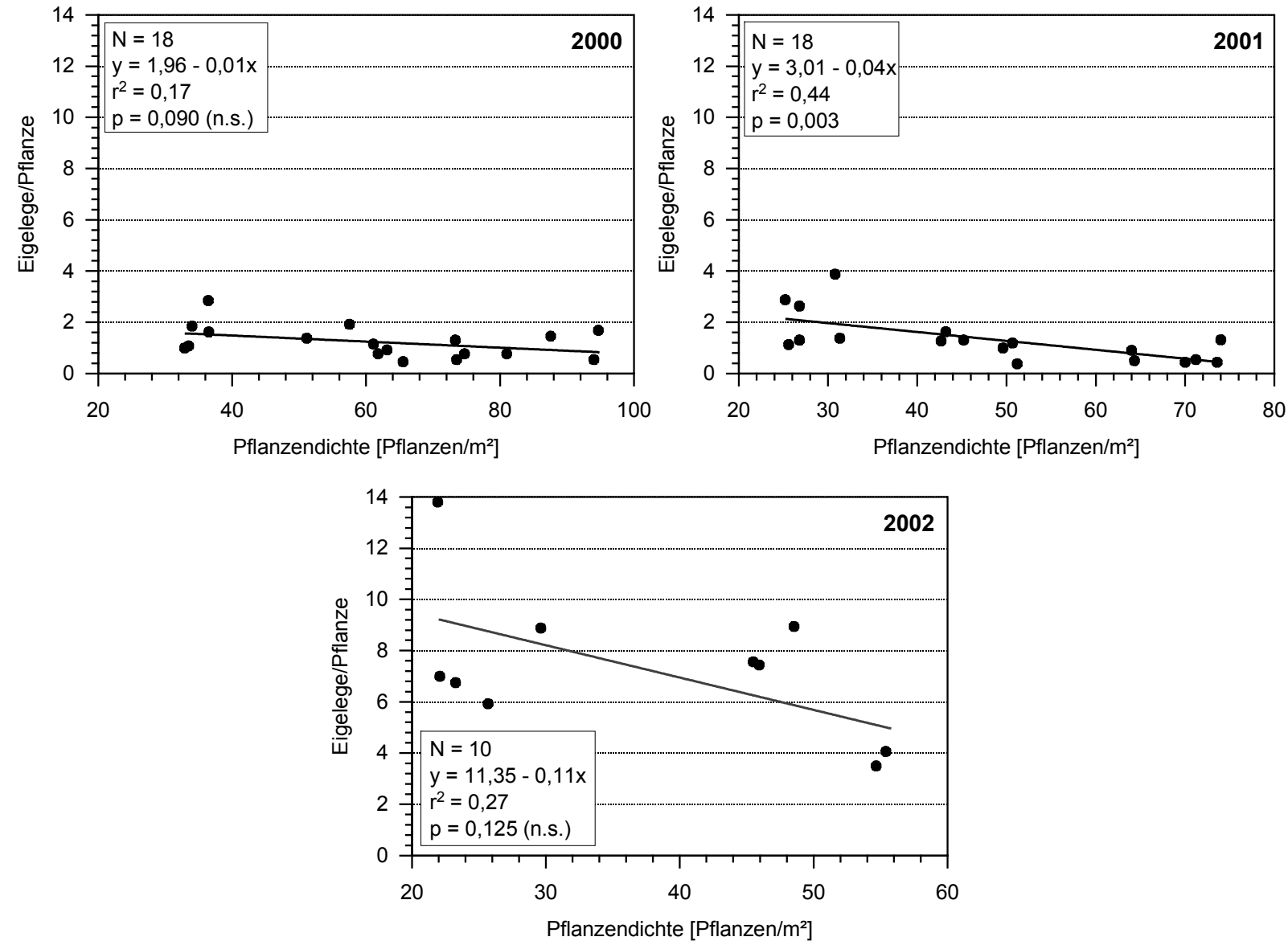

Abb. 30: Abundanz der Eigelege/Pflanze von C. napi in Anhängigkeit von der Pflanzendichte im April 2000 und 2001 am Standort Weendelsbreite und im April 2002 am Standort Otto-Hahn-Straße

Tab. 38: Korrelationen zwischen der Abundanz der Eigelege von $C$. napi in den Haupt- und Seitentrieben und der Pflanzendichte im April 2000 und 2001 am Standort Weendelsbreite und im April 2002 am Standort Otto-Hahn-Straße

\begin{tabular}{ccccc}
\hline $\begin{array}{c}\text { Korrelierende } \\
\text { Merkmale }\end{array}$ & Jahr & $r$ & $p$ & B [\%] \\
\hline Eigelege von C. napi in den Haupttrieben - Pflanzendichte & 2000 & $-0,30$ & 0,227 & 8,9 \\
& 2001 & $-0,62$ & 0,006 & 38,5 \\
& 2002 & $-0,41$ & 0,232 & 17,2 \\
Eigelege von C. napi in den Seitentrieben - Pflanzendichte & 2000 & $-0,44$ & 0,062 & 19,7 \\
& 2001 & $-0,55$ & 0,018 & 30,0 \\
& 2002 & $-0,83$ & 0,003 & 69,5 \\
\hline
\end{tabular}




\subsubsection{Beziehung zwischen der Anzahl der Eigelege/Pflanze von $C$. napi und der Pflan- zenarchitektur}

In Tabelle 39 sind die Beziehungen zwischen der Anzahl der Eigelege/Pflanze von C. napi und den untersuchten Wachstumsparametern der Pflanzen im April der drei Versuchsjahre dargestellt. Für die einzelnen Pflanzenparameter konnte nur im Versuch 2001 eine signifikante lineare Beziehung zur Dichte der Eigelege nachgewiesen werden. Diese Beziehung bestand für alle untersuchten Pflanzenparameter. Mit zunehmender Größe der Pflanzen kam es zu einer Zunahme des Einzelpflanzenbefalls. Die Zahl der Eigelege/Pflanze wies die stärkste positive Beziehung zum Parameter Anzahl Blätter auf (B=66,8 \%). Für die Parameter Wurzelhalsdurchmesser, Sprosslänge, Seitentrieblängen-Klassen und Durchmesser der Seitentriebe wurden Bestimmtheitsmaße von etwa 40 \%, für die Parameter Anzahl Seitentriebe und Länge der Seitentriebe von $26 \%$ bzw. $32 \%$ erreicht.

Tab. 39: Korrelationen zwischen der Abundanz der Eigelege/Pflanze von C. napi und den Wachstumsparametern der Pflanzen im April 2000 und 2001 am Standort Weendelsbreite und im April 2002 am Standort Otto-Hahn-Straße

\begin{tabular}{|c|c|c|c|c|}
\hline $\begin{array}{l}\text { Korrelierende } \\
\text { Merkmale }\end{array}$ & Jahr & $\mathrm{r}$ & $\mathrm{p}$ & $B$ [\%] \\
\hline $\begin{array}{r}\text { Eigelege von C. napi/Pflanze - Sprossdurchmesser } \\
\text { am Wurzelhals }\end{array}$ & $\begin{array}{l}2000 \\
2001 \\
2002\end{array}$ & $\begin{array}{l}+0,38 \\
+0,65 \\
+0,32\end{array}$ & $\begin{array}{l}0,115 \\
0,004 \\
0,376\end{array}$ & $\begin{array}{l}14,6 \\
41,7 \\
10,0\end{array}$ \\
\hline Eigelege von C. napi/Pflanze - Anzahl Blätter & $\begin{array}{l}2000 \\
2001 \\
2002\end{array}$ & $\begin{array}{l}+0,27 \\
+0,82 \\
+0,38\end{array}$ & $\begin{array}{l}0,270 \\
0,000 \\
0,276\end{array}$ & $\begin{array}{c}7,5 \\
66,8 \\
14,6\end{array}$ \\
\hline Eigelege von C. napi/Pflanze - Sprosslänge & $\begin{array}{l}2000 \\
2001 \\
2002\end{array}$ & $\begin{array}{l}+0,21 \\
+0,66 \\
+0,12\end{array}$ & $\begin{array}{l}0,404 \\
0,003 \\
0,732\end{array}$ & $\begin{array}{c}4,5 \\
43,3 \\
1,5\end{array}$ \\
\hline Eigelege von C. napi/Pflanze - Anzahl Seitentriebe & $\begin{array}{l}2000 \\
2001 \\
2002\end{array}$ & $\begin{array}{l}+0,43 \\
+0,51 \\
+0,26\end{array}$ & $\begin{array}{l}0,074 \\
0,031 \\
0,477\end{array}$ & $\begin{array}{c}18,3 \\
25,6 \\
6,7\end{array}$ \\
\hline $\begin{array}{c}\text { Eigelege von C. napi/Pflanze - Seitentrieblängen- } \\
\text { Klassen }\end{array}$ & $\begin{array}{l}2000 \\
2001 \\
2002\end{array}$ & $\begin{array}{c}+0,42 \\
+0,61 \\
-\end{array}$ & $\begin{array}{l}0,082 \\
0,007\end{array}$ & $\begin{array}{c}1,7 \\
37,8\end{array}$ \\
\hline $\begin{aligned} \text { Eigelege von C. napi/Pflanze - } & \text { Länge der } \\
& \text { Seitentriebe }\end{aligned}$ & $\begin{array}{l}2000 \\
2001 \\
2002\end{array}$ & $\begin{array}{c}- \\
+0,56 \\
+0,46\end{array}$ & $\begin{array}{l}0,014 \\
0,176\end{array}$ & $\begin{array}{l}31,7 \\
21,4\end{array}$ \\
\hline $\begin{aligned} \text { Eigelege von C. napi/Pflanze - } & \begin{array}{l}\text { Durchmesser der } \\
\text { Seitentriebe }\end{array}\end{aligned}$ & $\begin{array}{l}2000 \\
2001 \\
2002\end{array}$ & $\begin{array}{c}- \\
+0,64 \\
+0,59\end{array}$ & $\begin{array}{l}0,004 \\
0,069\end{array}$ & $\begin{array}{l}40,8 \\
35,1\end{array}$ \\
\hline
\end{tabular}

Bei getrennter Betrachtung der Haupt- und Seitentriebe ergaben sich im Versuch 2001 jeweils ähnliche Korrelationen zwischen der Dichte der Eigelege von C. napi und den Pflanzenparametern wie für die gesamte Pflanze (Tab. 40). Nur bei der Eiablage in die Haupttriebe ergab sich keine signifikante Beziehung zur Anzahl der Seitentriebe und bei der Eiablage in die Sei- 
tentriebe keine signifikante Beziehung zur Sprosslänge und zum Durchmesser der Seitentriebe. In den Versuchen 2000 und 2002 zeigten sich lediglich für die Eiablage in die Seitentriebe signifikante Korrelationen zu den geprüften Pflanzenparametern. Nur die Parameter Anzahl Blätter (in beiden Versuchen) sowie Sprosslänge (im Versuch 2002) wiesen keine statistisch gesicherten Beziehungen zur Anzahl der Eigelege in den Seitentrieben auf.

Tab. 40: Korrelationen zwischen der Abundanz der Eigelege von $C$. napi in den Haupt- und Seitentrieben und den Wachstumsparametern der Pflanzen im April 2000 und 2001 am Standort Weendelsbreite und im April 2002 am Standort Otto-Hahn-Straße

\begin{tabular}{|c|c|c|c|c|}
\hline $\begin{array}{l}\text { Korrelierende } \\
\text { Merkmale }\end{array}$ & Jahr & $r$ & $\mathrm{p}$ & $\mathrm{B}[\%]$ \\
\hline $\begin{aligned} \text { Eigelege von C. napi in den Haupttrieben } & \text { - Sprossdurchmesser } \\
& \text { am Wurzelhals }\end{aligned}$ & $\begin{array}{l}2000 \\
2001 \\
2002\end{array}$ & $\begin{array}{l}+0,15 \\
+0,60 \\
+0,22\end{array}$ & $\begin{array}{l}0,565 \\
0,008 \\
0,551\end{array}$ & $\begin{array}{c}2,2 \\
36,1 \\
4,7\end{array}$ \\
\hline $\begin{array}{c}\text { Eigelege von C. napi in den Seitentrieben - Sprossdurchmesser } \\
\text { am Wurzelhals }\end{array}$ & $\begin{array}{l}2000 \\
2001 \\
2002 \\
\end{array}$ & $\begin{array}{r}+0,72 \\
+0,54 \\
+0,68 \\
\end{array}$ & $\begin{array}{l}0,001 \\
0,020 \\
0,030 \\
\end{array}$ & $\begin{array}{l}51,3 \\
29,0 \\
45,8 \\
\end{array}$ \\
\hline Eigelege von C. napi in den Haupttrieben - Anzahl Blätter & $\begin{array}{l}2000 \\
2001 \\
2002\end{array}$ & $\begin{array}{l}+0,20 \\
+0,75 \\
+0,31\end{array}$ & $\begin{array}{l}0,429 \\
0,001 \\
0,392\end{array}$ & $\begin{array}{c}4,0 \\
55,7 \\
9,4\end{array}$ \\
\hline Eigelege von C. napi in den Seitentrieben - Anzahl Blätter & $\begin{array}{l}2000 \\
2001 \\
2002 \\
\end{array}$ & $\begin{array}{r}+0,30 \\
+0,71 \\
+0,62\end{array}$ & $\begin{array}{l}0,228 \\
0,001 \\
0,055\end{array}$ & $\begin{array}{c}8,9 \\
51,0 \\
38,2\end{array}$ \\
\hline Eigelege von C. napi in den Haupttrieben - Sprosslänge & $\begin{array}{l}2000 \\
2001 \\
2002\end{array}$ & $\begin{array}{r}-0,00 \\
+0,65 \\
+0,04\end{array}$ & $\begin{array}{l}0,986 \\
0,004 \\
0,920\end{array}$ & $\begin{array}{c}0,0 \\
42,4 \\
0,1\end{array}$ \\
\hline Eigelege von C. napi in den Seitentrieben - Sprosslänge & $\begin{array}{l}2000 \\
2001 \\
2002 \\
\end{array}$ & $\begin{array}{r}+0,59 \\
+0,44 \\
+0,49\end{array}$ & $\begin{array}{l}0,010 \\
0,063 \\
0,147 \\
\end{array}$ & $\begin{array}{l}34,9 \\
19,6 \\
24,2 \\
\end{array}$ \\
\hline Eigelege von C. napi in den Haupttrieben - Anzahl Seitentriebe & $\begin{array}{l}2000 \\
2001 \\
2002\end{array}$ & $\begin{array}{r}+0,26 \\
+0,45 \\
+0,16\end{array}$ & $\begin{array}{l}0,301 \\
0,056 \\
0,668\end{array}$ & $\begin{array}{c}6,7 \\
20,6 \\
2,5\end{array}$ \\
\hline Eigelege von C. napi in den Seitentrieben - Anzahl Seitentriebe & $\begin{array}{l}2000 \\
2001 \\
2002 \\
\end{array}$ & $\begin{array}{r}+0,59 \\
+0,47 \\
+0,65\end{array}$ & $\begin{array}{l}0,009 \\
0,046 \\
0,041\end{array}$ & $\begin{array}{l}35,1 \\
22,2 \\
42,1 \\
\end{array}$ \\
\hline $\begin{array}{c}\text { Eigelege von C. napi in den Haupttrieben - Seitentrieblängen- } \\
\text { Klassen }\end{array}$ & $\begin{array}{l}2000 \\
2001 \\
2002\end{array}$ & $\begin{array}{c}+0,17 \\
+0,53 \\
-\end{array}$ & $\begin{array}{l}0,508 \\
0,023\end{array}$ & $\begin{array}{r}2,9 \\
28,0\end{array}$ \\
\hline $\begin{array}{c}\text { Eigelege von C. napi in den Seitentrieben - Seitentrieblängen- } \\
\text { Klassen }\end{array}$ & $\begin{array}{l}2000 \\
2001 \\
2002 \\
\end{array}$ & $\begin{array}{c}+0,76 \\
+0,63 \\
- \\
\end{array}$ & $\begin{array}{l}0,000 \\
0,005\end{array}$ & $\begin{array}{l}58,2 \\
39,9\end{array}$ \\
\hline $\begin{array}{r}\text { Eigelege von C. napi in den Haupttrieben - Länge der } \\
\text { Seitentriebe }\end{array}$ & $\begin{array}{l}2000 \\
2001 \\
2002\end{array}$ & $\begin{array}{c}- \\
+0,49 \\
+0,35\end{array}$ & $\begin{array}{l}0,037 \\
0,320\end{array}$ & $\begin{array}{l}24,0 \\
12,4\end{array}$ \\
\hline $\begin{array}{r}\text { Eigelege von C. napi in den Seitentrieben - Länge der } \\
\text { Seitentriebe }\end{array}$ & $\begin{array}{l}2000 \\
2001 \\
2002 \\
\end{array}$ & $\begin{array}{c}- \\
+0,56 \\
+0,84 \\
\end{array}$ & $\begin{array}{l}0,014 \\
0,003\end{array}$ & $\begin{array}{l}31,8 \\
70,9\end{array}$ \\
\hline $\begin{array}{c}\text { Eigelege von C. napi in den Haupttrieben - Durchmesser der } \\
\text { Seitentriebe }\end{array}$ & $\begin{array}{l}2000 \\
2001 \\
2002\end{array}$ & $\begin{array}{c}- \\
+0,63 \\
+0,49\end{array}$ & $\begin{array}{l}0,005 \\
0,146\end{array}$ & $\begin{array}{l}39,5 \\
24,2\end{array}$ \\
\hline $\begin{array}{c}\text { Eigelege von C. napi in den Seitentrieben - Durchmesser der } \\
\text { Seitentriebe }\end{array}$ & $\begin{array}{l}2000 \\
2001 \\
2002\end{array}$ & $\begin{array}{l}- \\
+0,44 \\
+0,89\end{array}$ & $\begin{array}{l}0,063 \\
0,001\end{array}$ & $\begin{array}{l}19,6 \\
78,8\end{array}$ \\
\hline
\end{tabular}


Die Dichte der Eigelege in den Seitentrieben zeigte mit einem Bestimmtheitsmaß von $50 \%$ bis $80 \%$ die engsten Zusammenhänge mit den Parametern Wurzelhalsdurchmesser und Seitentrieblängen-Klassen im Versuch 2000 und zum Durchmesser der Seitentriebe und zur Länge der Seitentriebe im Versuch 2002. Für den Parameter Anzahl Seitentriebe wurde in beiden Versuchen eine etwas schwächer korrelierte Beziehung mit einem Bestimmtheitsmaß von 35,1 \% bzw. 42,1 \% zur Befallsdichte in den Seitentrieben erreicht.

\subsubsection{Beziehung zwischen der Anzahl der Eigelege/Pflanze von $C$. pallidactylus und der Pflanzendichte}

Die Beziehung zwischen der Anzahl der Eigelege/Pflanze von C. pallidactylus und der Pflanzendichte wurde nur im April der Versuchsjahre 2000 und 2002 überprüft, da die Anzahl der Eiablagestellen im April 2001 zu gering war.

In beiden Versuchsjahren ergab sich ein signifikanter linearer Zusammenhang zwischen der Anzahl der Eigelege/Pflanze von C. pallidactylus und der Pflanzendichte (Abb. 31). Eine Abnahme der Pflanzendichte führte zu einem Anstieg der Anzahl der Eigelege/Pflanze. Bei höherem Befallsdruck im Versuch 2002 war eine stärkere Steigung der Regressionsgeraden $(b=$ 0,10) zu beobachten als bei niedrigem Befallsdruck im Versuch $2000(\mathrm{~b}=0,02)$. Der Zusammenhang zwischen der Abundanz der Eigelege/Pflanze und der Pflanzendichte war im Versuch $2000(B=57 \%)$ deutlich enger als im Versuch $2002(B=39 \%)$.
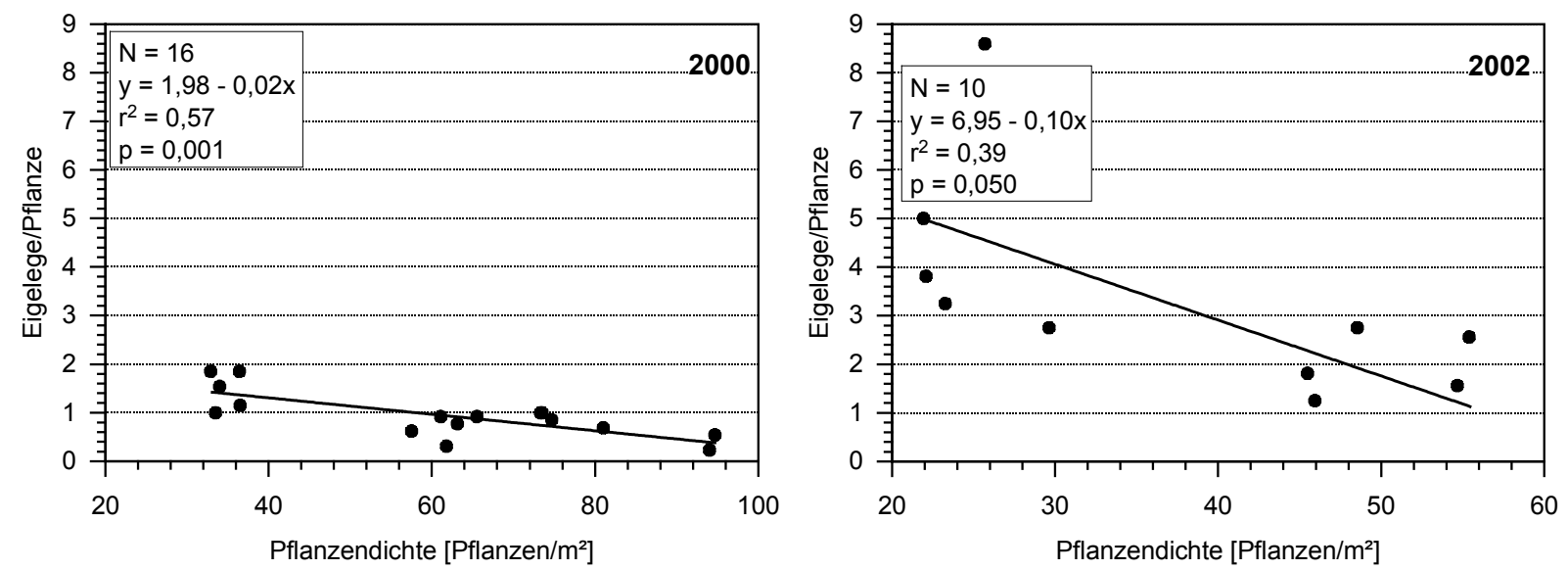

Abb. 31: Abundanz der Eigelege/Pflanze von C. pallidactylus in Anhängigkeit von der Pflanzendichte und der im April 2000 und 2002 an den Standorten Weendelsbreite bzw. Otto-Hahn-Straße 


\subsubsection{Beziehung zwischen der Anzahl der Eigelege/Pflanze von $C$. pallidactylus und der Pflanzenarchitektur}

Die Beziehung zwischen der Anzahl der Eigelege/Pflanze von C. pallidactylus und der Pflanzenarchitektur wurde ebenfalls nur im April der Versuchsjahre 2000 und 2002 überprüft, da die Anzahl der Eiablagestellen im April 2001 zu gering war.

Im Versuch 2000 ergab sich mit Ausnahme der Parameter Durchmesser der Seitentriebe und Länge der Seitentriebe eine signifikante lineare Korrelation zur Dichte der Eigelege/Pflanze (Tab. 41). Mit einer Zunahme des Wurzelhalsdurchmessers, der Sprosslänge, der Anzahl Blätter und Seitentriebe und der Seitentrieblängen-Klassen stieg der Einzelpflanzenbefall deutlich an. Im Versuch 2002 konnten lediglich für die Merkmale Wurzelhalsdurchmesser und Anzahl Seitentriebe signifikante Korrelationen nachgewiesen werden. Für die Parameter Wurzelhalsdurchmesser und Anzahl Seitentriebe (in beiden Versuchen) sowie für den Parameter Seitentrieblängen-Klasse (Versuch 2000) wurden die engsten Korrelationen mit einem Bestimmtheitsmaß von $54,8 \%$ bis $65,6 \%$ erreicht.

Tab. 41: Korrelationen zwischen der Abundanz der Eigelege/Pflanze von C. pallidactylus und den Wachstumsparametern der Pflanzen im April 2000 und 2002 an den Standorten Weendelsbreite bzw. Otto-Hahn-Straße

\begin{tabular}{|c|c|c|c|c|c|}
\hline $\begin{array}{c}\text { Korrelierende } \\
\text { Merkmale }\end{array}$ & & Jahr & $r$ & $\mathrm{p}$ & $\mathrm{B}[\%]$ \\
\hline Eigelege von C. pallidactylus /Pflanze & $\begin{array}{l}\text { - Sprossdurchmesser } \\
\text { am Wurzelhals }\end{array}$ & $\begin{array}{l}2000 \\
2002\end{array}$ & $\begin{array}{l}+0,81 \\
+0,74\end{array}$ & $\begin{array}{l}0,000 \\
0,014\end{array}$ & $\begin{array}{l}65,6 \\
54,8\end{array}$ \\
\hline Eigelege von C. pallidactylus/Pflanze & - Anzahl Blätter & $\begin{array}{l}2000 \\
2002\end{array}$ & $\begin{array}{l}+0,65 \\
+0,58\end{array}$ & $\begin{array}{l}0,008 \\
0,078\end{array}$ & $\begin{array}{l}40,5 \\
33,4\end{array}$ \\
\hline Eigelege von C. pallidactylus/Pflanze & - Sprosslänge & $\begin{array}{l}2000 \\
2002\end{array}$ & $\begin{array}{l}+0,62 \\
+0,31\end{array}$ & $\begin{array}{l}0,011 \\
0,380\end{array}$ & $\begin{array}{c}37,8 \\
9,8\end{array}$ \\
\hline Eigelege von C. pallidactylus/Pflanze & - Anzahl Seitentriebe & $\begin{array}{l}2000 \\
2002\end{array}$ & $\begin{array}{l}+0,80 \\
+0,77\end{array}$ & $\begin{array}{l}0,000 \\
0,009\end{array}$ & $\begin{array}{l}64,5 \\
59,9\end{array}$ \\
\hline Eigelege von C. pallidactylus/Pflanze & $\begin{array}{l}\text { - Seitentrieblängen- } \\
\text { Klassen }\end{array}$ & $\begin{array}{l}2000 \\
2002\end{array}$ & $\begin{array}{c}0,79 \\
-\end{array}$ & 0,000 & 62,5 \\
\hline Eigelege von C. pallidactylus/Pflanze & $\begin{array}{l}\text { - Länge der } \\
\text { Seitentriebe }\end{array}$ & $\begin{array}{l}2000 \\
2002\end{array}$ & $+0,52$ & 0,122 & 27,0 \\
\hline Eigelege von C. pallidactylus/Pflanze & $\begin{array}{l}\text { - Durchmesser der } \\
\text { Seitentriebe }\end{array}$ & $\begin{array}{l}2000 \\
2002\end{array}$ & +- & 0,124 & 26,7 \\
\hline
\end{tabular}




\subsubsection{Räumliche Verteilung der Eier und Larven von $C$. napi und $C$. pallidactylus in den Rapspflanzen}

\subsubsection{Vertikale Verteilung der Eier von $C$. napi auf verschiedene Abschnitte des Haupttriebes}

Im April der Versuchsjahre 2001 und 2002 wurde die Zahl der Eier von C. napi in definierten vertikalen Abschnitten des Haupttriebes (Triebhöhe: 0-20 cm, 20-40 cm und über $40 \mathrm{~cm}$ ) untersucht. Mit Hilfe des Covariate(s)-Tests der ANOVA zeigte sich in beiden Versuchen, dass die Eiablage in verschiedenen Stängelhöhen nicht durch die Saatstärke beeinflusst wurde (Saatstärke als Covariate und Klassen der Triebhöhe als Variate, 2001: $F=0,099, p=0,755$ bzw. $F=16,206, p=0,000,2002: F=0,022, p=0,882$ bzw. $F=32,932, p=0,000$ ).

Bei allen Saatstärken befand sich der größte Anteil der Eier in dem oberen Abschnitt mit der Triebspitze (Abb. 32 und 33). Bei den längeren Pflanzen der Saatstärke $30 \mathrm{Kö.} / \mathrm{m}^{2}$ handelte es sich dabei um den Bereich über $40 \mathrm{~cm}$, bei den mittellangen Pflanzen der Saatstärke $60 \mathrm{Kö.} / \mathrm{m}^{2}$ um die Bereiche 20-40 cm und über $40 \mathrm{~cm}$ und bei den kürzeren Pflanzen der Saatstärke 90 Kö./m² um den Bereich 20-40 cm. Die Länge der Pflanzen lag im April 2001 in den Saatstärken 30, 60 und $90 \mathrm{Kö.} / \mathrm{m}^{2}$ im Mittel ( \pm SE) bei $62,2 \pm 0,8 \mathrm{~cm}, 55,6 \pm 0,7 \mathrm{~cm}$ bzw. 45,6 \pm 0,8 $\mathrm{cm}$ und im April 2002 in den Saatstärken 30 und $60 \mathrm{Kö.} / \mathrm{m}^{2}$ bei 56,7 $\pm 1,6 \mathrm{~cm}$ bzw. 49,7 $\pm 1,6$ $\mathrm{cm}$.

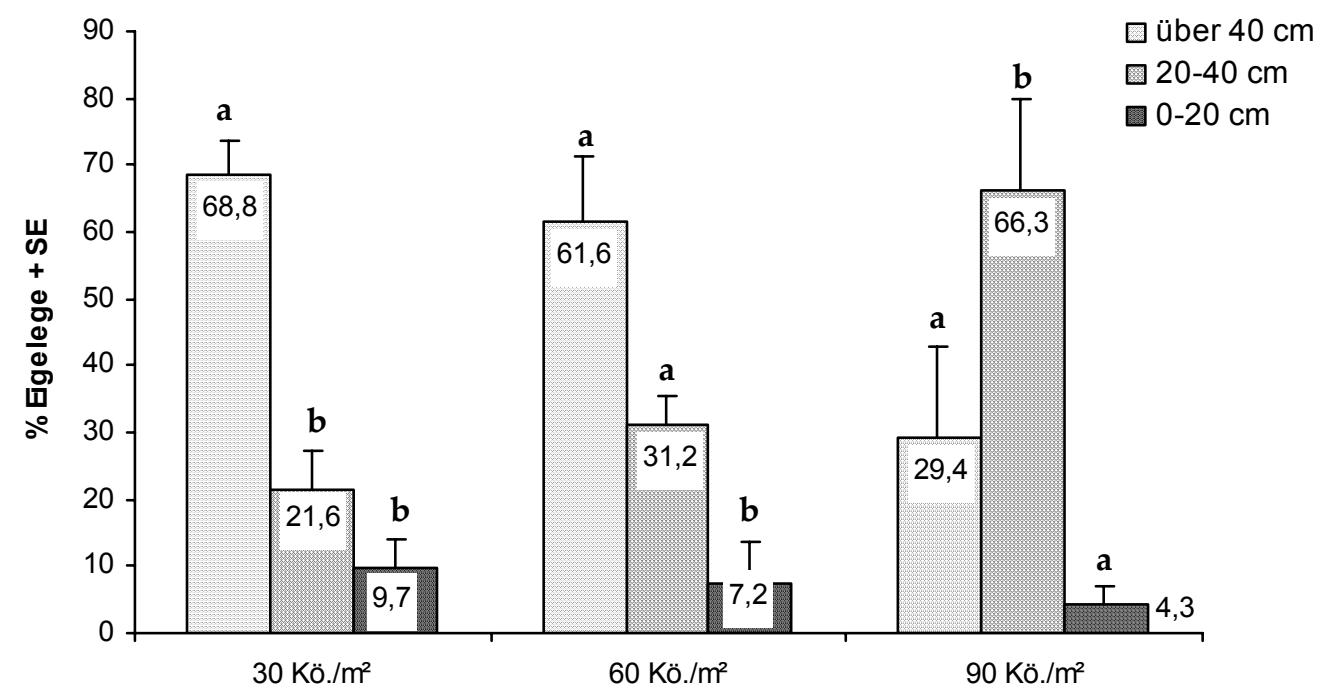

Abb. 32: Verteilung der Eier von $C$. napi auf verschiedene Höhenabschnitte in Pflanzen unterschiedlicher Saatstärken im April 2001 am Standort Weendelsbreite (Unterschiedliche Buchstaben stellen signifikante Unterschiede innerhalb der Säulengruppe dar, ARCSIN-Transformation, ANOVA, Tukey-Test, $\mathrm{p}<0,05$ ) 


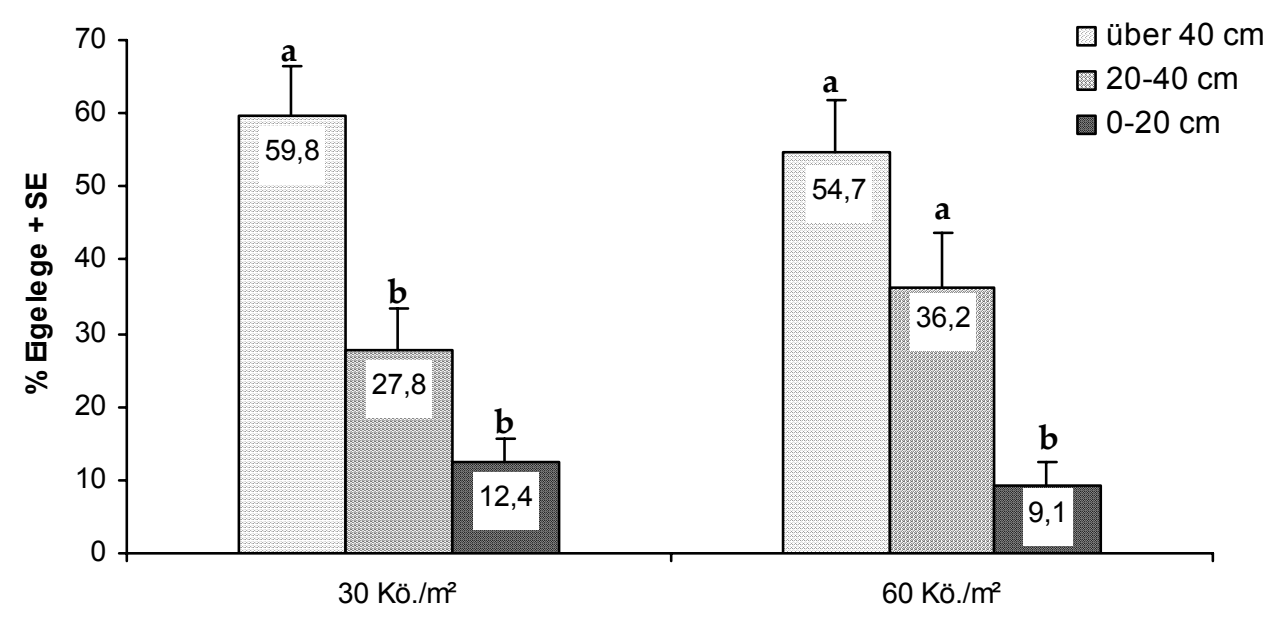

Abb. 33: Verteilung der Eier von C. napi auf verschiedene Höhenabschnitte in Pflanzen unterschiedlicher Saatstärken im April 2002 am Standort Otto-Hahn-Straße (Unterschiedliche Buchstaben stellen signifikante Unterschiede innerhalb der Säulengruppe dar, ARCSIN-Transformation, Kruskal-Wallis-Test, $\mathrm{p}<0,05$ )

\subsubsection{Verteilung der Eier und Larven von $C$. napi auf Haupt- und Seitentriebe}

Der Befall von C. napi verteilte sich auf die Haupt- und Seitentriebe der Einzelpflanzen (Abb. 34, 35 und 36). Die Seitentriebe wurden erst ab einem Durchmesser von 1,7 mm mit Eiern belegt. Es ist zu erkennen, dass der Anteil der Eier und Larven in den heranwachsenden Seitentrieben im Mai aller drei Jahre gegenüber dem Vormonat April angestiegen war. Die Eiablage war demnach bei den ersten Probeterminen im April noch nicht abgeschlossen. Der Vergleich der Saatvarianten bezüglich des Seitentriebbefalls zeigt in den Jahren 2000 und 2001 nur im Monat Mai signifikante Unterschiede (Abb. 34 und 35): Der Anteil der Larven in den Seitentrieben war bei der Saatstärke $30 \mathrm{Kö.} / \mathrm{m}^{2}$ mit 19,9 \% bzw. 39,7 \% gegenüber der Saatstärke $90 \mathrm{Kö.} / \mathrm{m}^{2}$ mit 1,0 \% bzw. 11,2 \% signifikant erhöht. Im Versuch 2002 zeichnete sich in beiden Monaten ein signifikanter Unterschied zwischen den Saatstärken ab (Abb. 36). Der Vergleich der drei Jahre lässt erkennen, dass die Eiablage in die Seitentriebe bei der Saatstärke $30 \mathrm{Kö.} / \mathrm{m}^{2}$ im Mai 2001 und 2002 mit 39,7 \% bzw. 30,7 \% deutlich höher ausfiel als im Mai 2000 mit $19,9 \%$.

Bei den absoluten Werten für die Anzahl der Eier und Larven von C. napi in Haupt- und Seitentrieben in den Monaten April bzw. Mai der Versuche 2000 bis 2002 ergaben sich ähnliche Unterschiede zwischen den Saatstärken (Tab. A-4, Anhang). 


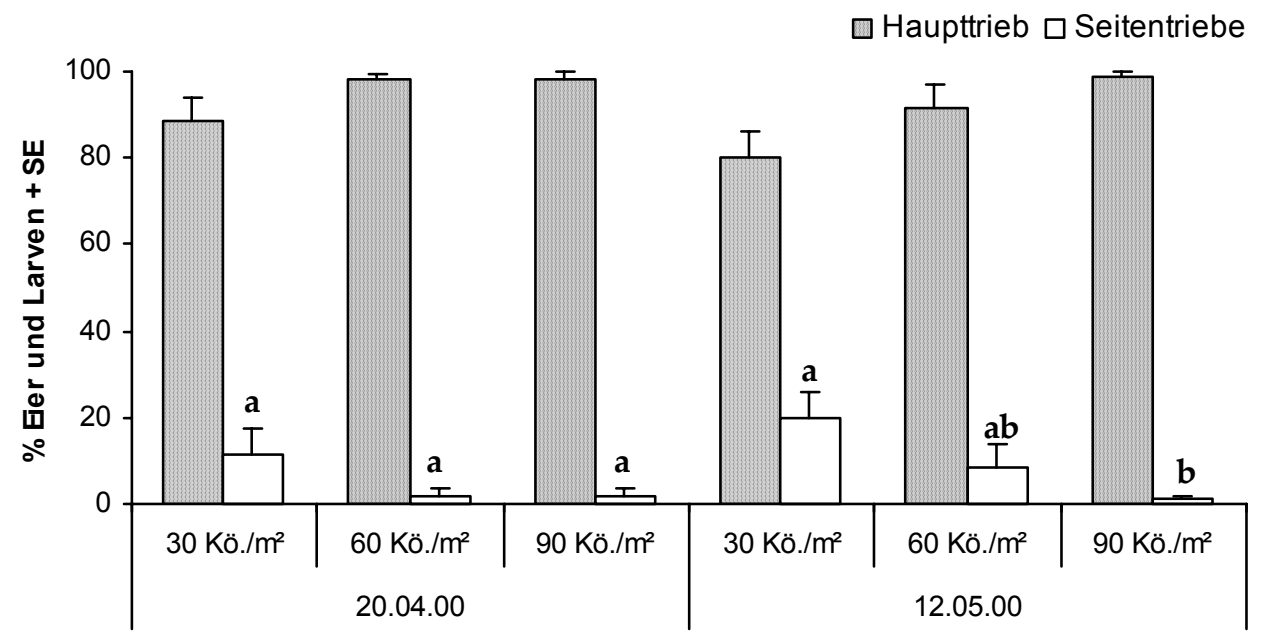

Abb. 34: Verteilung der Eier und Larven von C. napi auf Haupt- und Seitentriebe in unterschiedlichen Saatstärken im Jahr 2000 am Standort Weendelsbreite (Unterschiedliche Buchstaben indizieren signifikante Unterschiede zwischen den Saatstärken an einzelnen Terminen, ARCSIN-Transformation, ANOVA, Tukey-Test, $p<0,05$ )

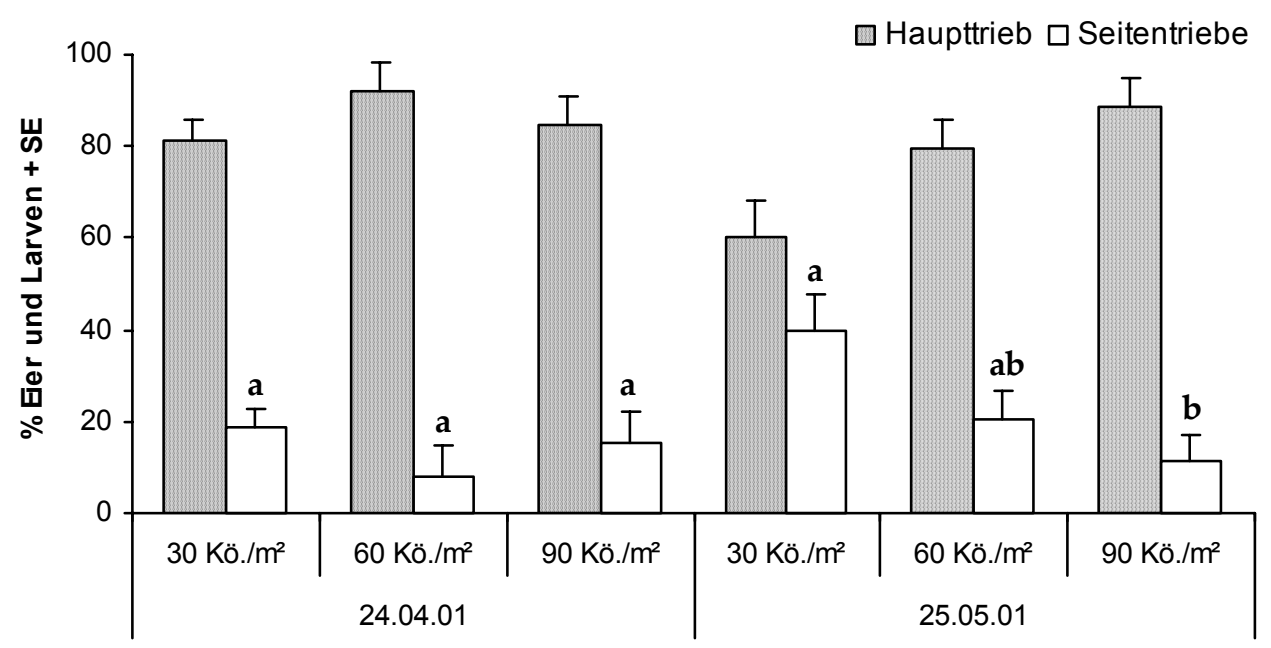

Abb. 35: Verteilung der Eier und Larven von C. napi auf Haupt- und Seitentriebe in unterschiedlichen Saatstärken im Jahr 2001 am Standort Weendelsbreite (Unterschiedliche Buchstaben indizieren signifikante Unterschiede zwischen den Saatstärken an einzelnen Terminen, ARCSIN-Transformation, ANOVA, Tukey-Test, $p<0,05$ ) 


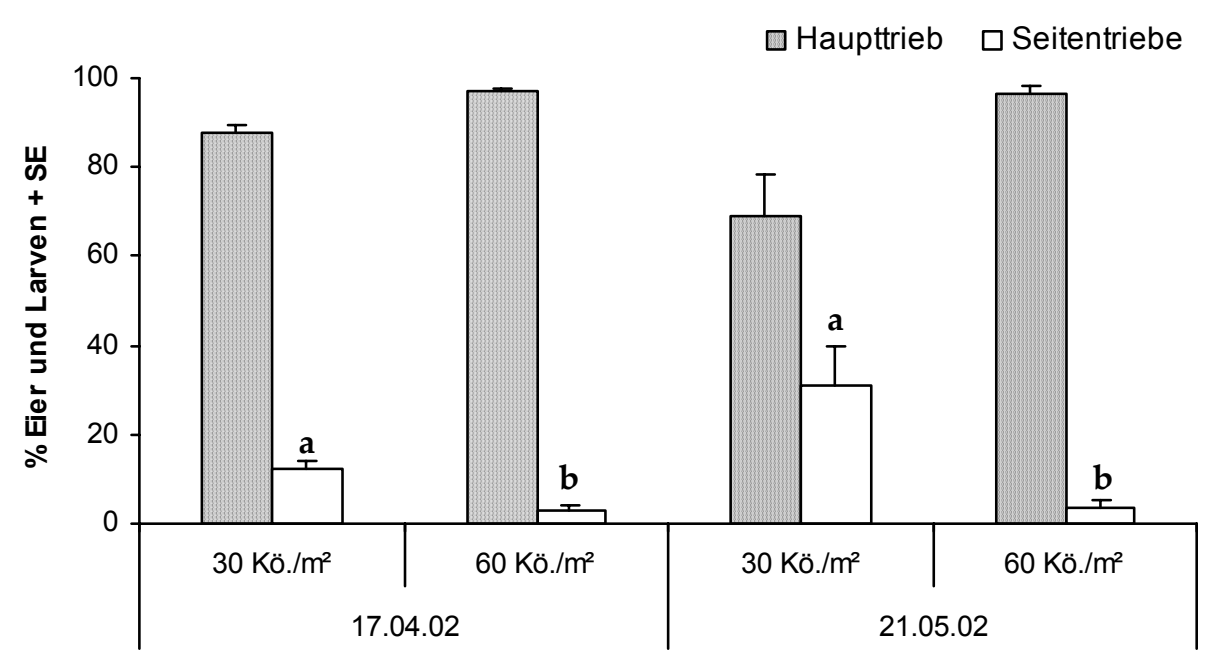

Abb. 36: Verteilung der Eier und Larven von $C$. napi auf Haupt- und Seitentriebe in unterschiedlichen Saatstärken im Jahr 2002 am Standort Otto-Hahn-Straße (Unterschiedliche Buchstaben indizieren signifikante Unterschiede zwischen den Saatstärken an einzelnen Terminen, ARCSIN-Transformation, Mann-Whitney U-Test, $p<0,05$ )

\subsubsection{Verteilung der Eigelege und Larven von $C$. pallidactylus auf Blätter und Triebe}

Die Verteilung der Eigelege von C. pallidactylus auf Blätter und Triebe wurde nur im April der Versuchsjahre 2000 und 2002 überprüft, da die Anzahl der Eigelege im April 2001 zu gering war. Im April 2002 zeigte sich, dass der größte Teil der Eier bei den beiden Saatstärken 30 und $60 \mathrm{Kö.} / \mathrm{m}^{2}$ in die Unterseite der Blätter abgelegt wurde, nur 17,1 \% bzw. 15,5\% der Eigelege waren auf der Oberseite der Blätter zu finden. Ein signifikanter Unterschied zwischen den Saatstärken konnte nicht nachgewiesen werden (Mann-Whitney U-Test, p = 0,251).

Die Untersuchung der Pflanzen im April der Jahre 2000 und 2002 ergab, dass der weitaus größte Teil der Eier in die Blätter der Haupttriebe abgelegt wurde (Abb. 37 und 38). Relativ wenige Eigelege fanden sich in den Blättern der Seitentriebe und nur sehr wenige in den Trieben selbst. Die Eiablage in die Blätter der Haupttriebe wies in beiden Untersuchungsjahren keine signifikanten Unterschiede zwischen den Saatvarianten auf. Eine Eiablage in die Blätter der Seitentriebe war lediglich im Versuch 2000 zu beobachten. Sie war bei der Saatstärke 30 Kö. $/ \mathrm{m}^{2}$ signifikant höher als bei den Saatstärken 60 und $90 \mathrm{Kö.} / \mathrm{m}^{2}$. Die Eiablage in die Haupttriebe wies in beiden Jahren keine signifikanten Unterschiede zwischen den Saatstärken auf. Eine Eiablage in die Seitentriebe war nur im Jahr 2002 festzustellen; die Unterschiede zwischen den Saatvarianten waren nicht signifikant. 


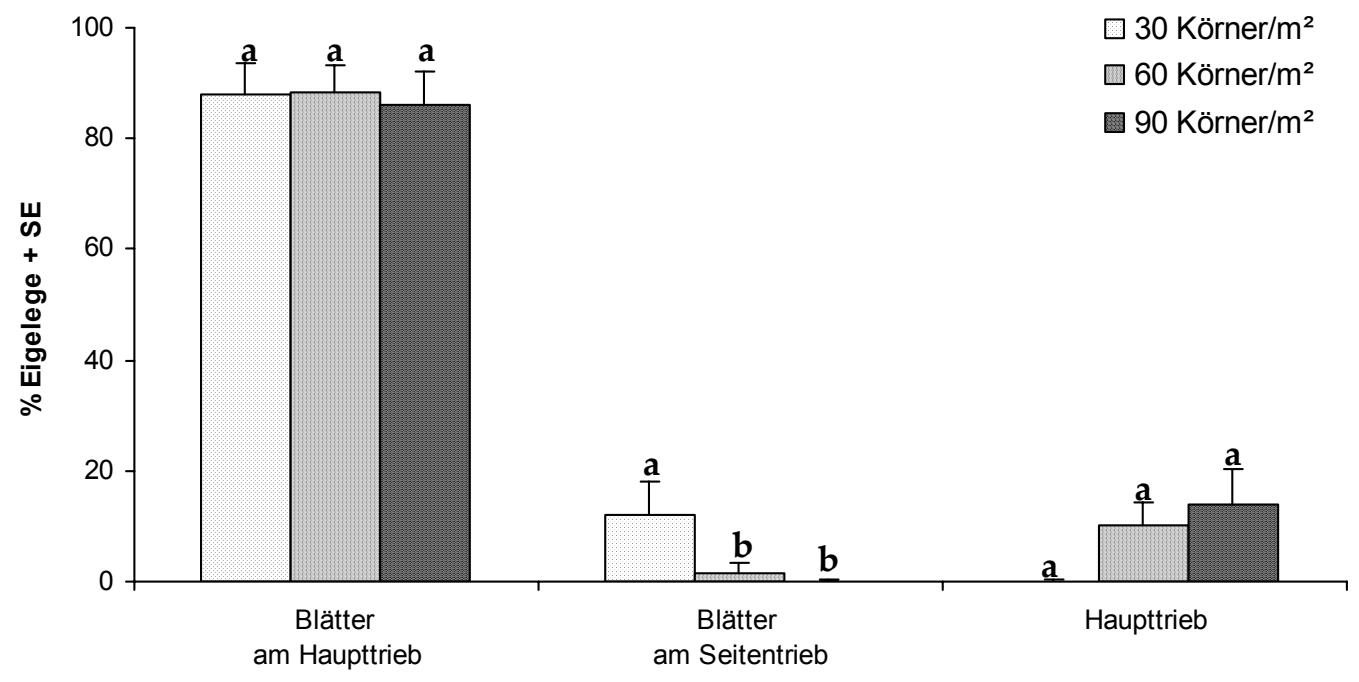

Abb. 37: Verteilung der Eigelege von $C$. pallidactylus auf Blätter und Triebe in Pflanzen unterschiedlicher Saatstärken im April 2000 am Standort Weendelsbreite (Unterschiedliche Buchstaben indizieren signifikante Unterschiede zwischen den Saatstärken, ARCSIN-Transformation, ANOVA, Tukey-Test, $\mathrm{p}<0,05$ )

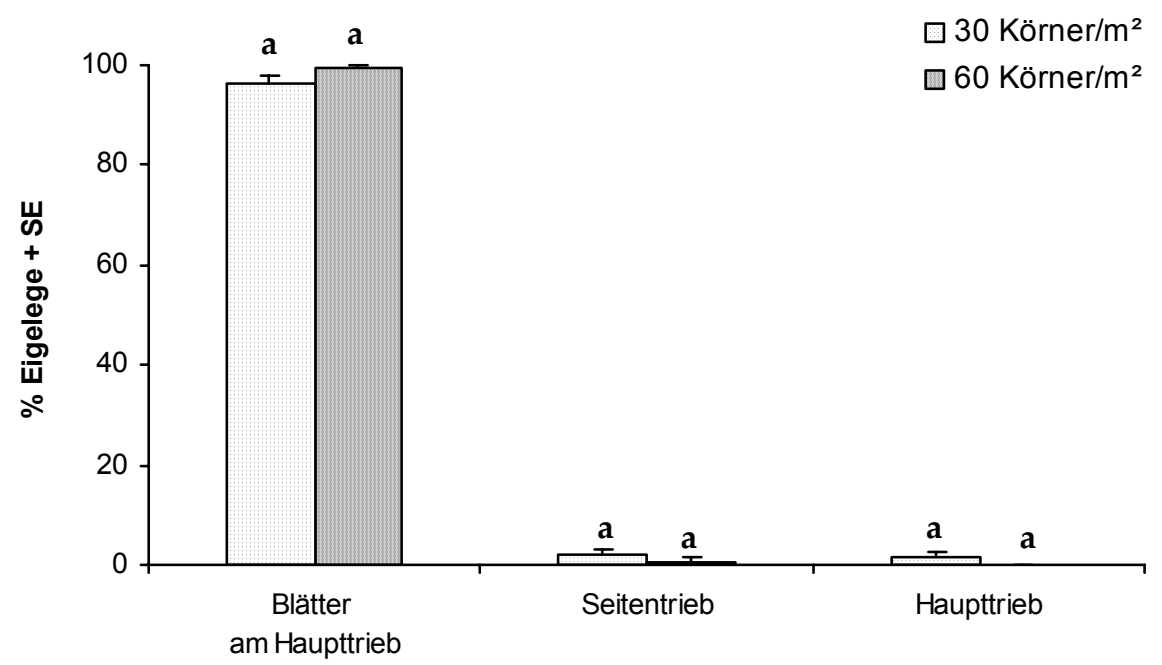

Abb. 38: Verteilung der Eigelege von C. pallidactylus auf Blätter und Triebe in Pflanzen verschiedener Saatstärken im April 2002 am Standort Otto-Hahn-Straße (Unterschiedliche Buchstaben indizieren signifikante Unterschiede zwischen den Saatstärken, ARCSIN-Transformation, Mann-Whitney U-Test, $\mathrm{p}<0,05$ )

Der im Vergleich zur Eiablage relativ starke Befall der Seitentriebe mit Larven von C. pallidactylus im Mai der drei Untersuchungsjahre weist auf eine spätere Eiablage in die heranwachsenden Seitentriebe hin (Abb. 39). Die Seitentriebe der Saatstärke $30 \mathrm{Kö.} / \mathrm{m}^{2}$ wurden dabei deutlich stärker bevorzugt als die Seitentriebe der Saatstärken 60 und 90 Kö./m². Der Anteil der Larven in den Seitentrieben war bei der Saatstärke $30 \mathrm{Kö.} / \mathrm{m}^{2} \mathrm{im} \mathrm{Jahr} 2000$ gegenüber 
der Saatstärke 90 Kö./m², im Jahr 2001 gegenüber den Saatstärken 60 und 90 Kö./m² sowie im Jahr 2002 gegenüber der Saatstärke $60 \mathrm{Kö} / \mathrm{m}^{2}$ signifikant erhöht. Bei dem Vergleich der drei Jahre wird deutlich, dass in den Jahren 2001 und 2002 gegenüber dem Jahr 2000 nicht nur der relative Befall der Seitentriebe drastisch anstieg, sondern auch die Unterschiede zwischen den einzelnen Saatstärken deutlich größer wurden.

Die absoluten Werte der Eigelege und des Larvenbefalls von C. pallidactylus an Blättern und Trieben wiesen in den Monaten April bzw. Mai der Versuche 2000 bis 2002 häufig signifikante Unterschiede zwischen den Saatstärken auf (Tab. A-5, Anhang).

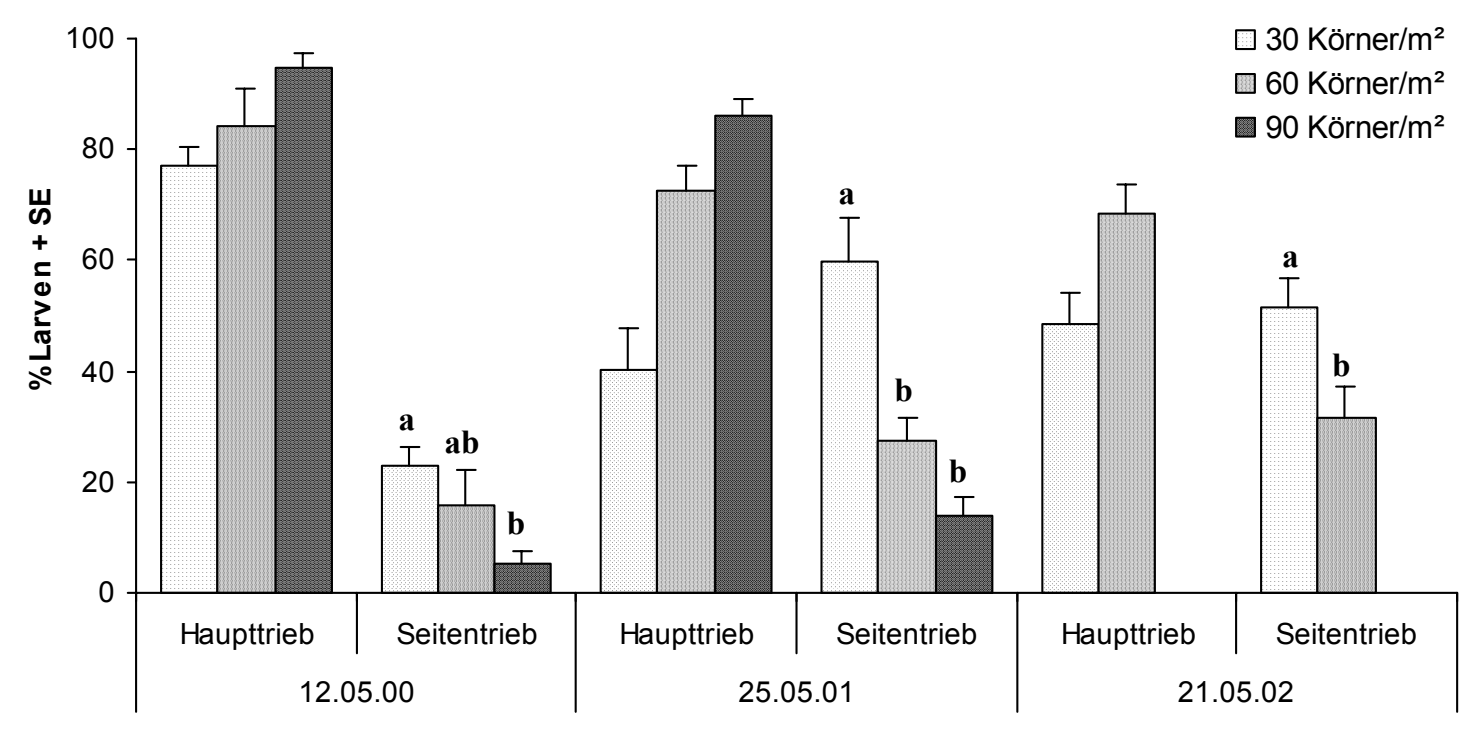

Abb. 39: Verteilung der Larven von C. pallidactylus auf Haupt- und Seitentriebe in Pflanzen unterschiedlicher Saatstärken im Mai 2000 und 2001 am Standort Weendelsbreite und im Mai 2002 am Standort Otto-Hahn-Straße (Unterschiedliche Buchstaben indizieren signifikante Unterschiede zwischen den Saatstärken innerhalb der Säulengruppe, ARCSIN-Transformation, 2000 und 2001: ANOVA, Tukey-Test, 2002: Mann-Whitney U-Test, $\mathrm{p}<0,05$ )

\subsubsection{Vertikale Verteilung der Eigelege von $C$. pallidactylus auf unterschiedliche Blattetagen des Haupttriebes}

Die vertikale Verteilung der Eigelege von C. pallidactylus auf verschiedene Blattetagen des Haupttriebes der Rapspflanzen wurde nur im April 2002 untersucht (Abb. 40). Innerhalb der Blattetagen wurde nicht zwischen den Haupt- und Lanzettblättern unterschieden. Die Eiablage von C. pallidactylus fand vorzugsweise in den unteren bis mittleren Blattetagen statt. Der höchste Befall entfiel bei der Saatstärke $30 \mathrm{Kö.} / \mathrm{m}^{2}$ auf die Blattetagen 5 bis 7 , bei der Saatstärke $60 \mathrm{Kö.} / \mathrm{m}^{2}$ auf die Blattetage 5. Bei der überwiegenden Zahl der Blattetagen war der 
Befall bei der Saatstärke $30 \mathrm{Kö.} / \mathrm{m}^{2}$ eindeutig höher als bei der Saatstärke $60 \mathrm{Kö.} / \mathrm{m}^{2}$. Dies war für die Blattpositionen 7 und 8 statistisch abzusichern.

Die größte Befallshäufigkeit war bei der Saatstärke $30 \mathrm{Kö.} / \mathrm{m}^{2}$ in den Blattetagen 3 bis 9 und bei der Saatstärke $60 \mathrm{Kö.} / \mathrm{m}^{2}$ in den Blattetagen 3 bis $10 \mathrm{zu}$ finden (Abb. 41). Die Blattpositionen 2-9 bzw. 2-6 wiesen mehr als nur eine Eiablagestelle/Blatt auf (Abb. 42). Die 5. Blattetage erreichte bei den Saatstärken 30 und $60 \mathrm{Kö.} / \mathrm{m}^{2}$ mit 1,7 bzw. 1,6 Eigelegen/befallenes Blatt den höchsten Wert. Die maximale Anzahl lag bei der Saatstärke $30 \mathrm{Kö.} / \mathrm{m}^{2}$ bei 6 Eigelegen pro Blatt. Bei der Saatstärke $60 \mathrm{Kö.} / \mathrm{m}^{2}$ wurden maximal 3 Eigelege pro Blatt erreicht.

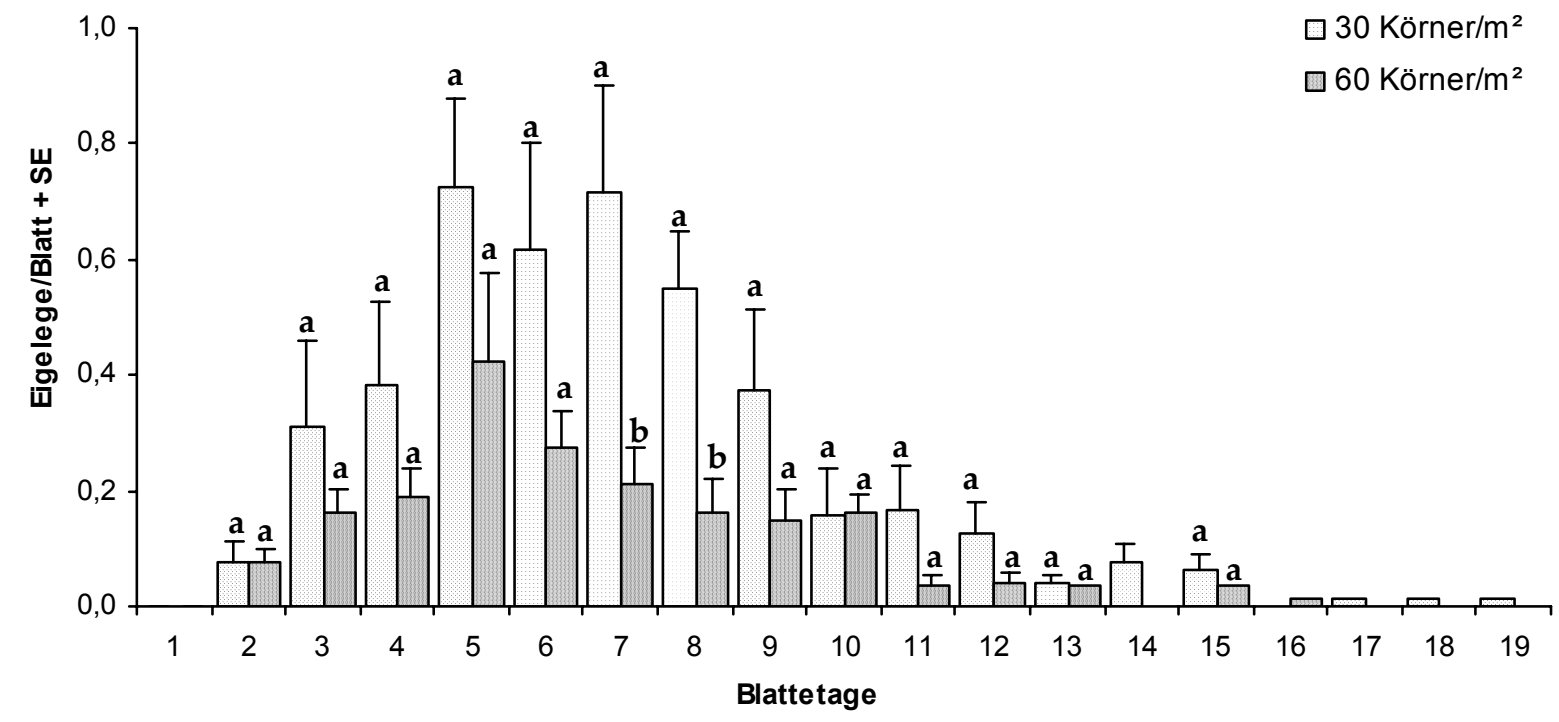

Abb. 40: Mittlere Anzahl der Eigelege/Blattetage von $C$. pallidactylus in Pflanzen unterschiedlicher Saatstärken im April 2002 am Standort Otto-Hahn-Straße (Unterschiedliche Buchstaben stellen signifikante Unterschiede zwischen den Saatstärken dar, Mann-Whitney U-Test, $\mathrm{p}<0,05$ )

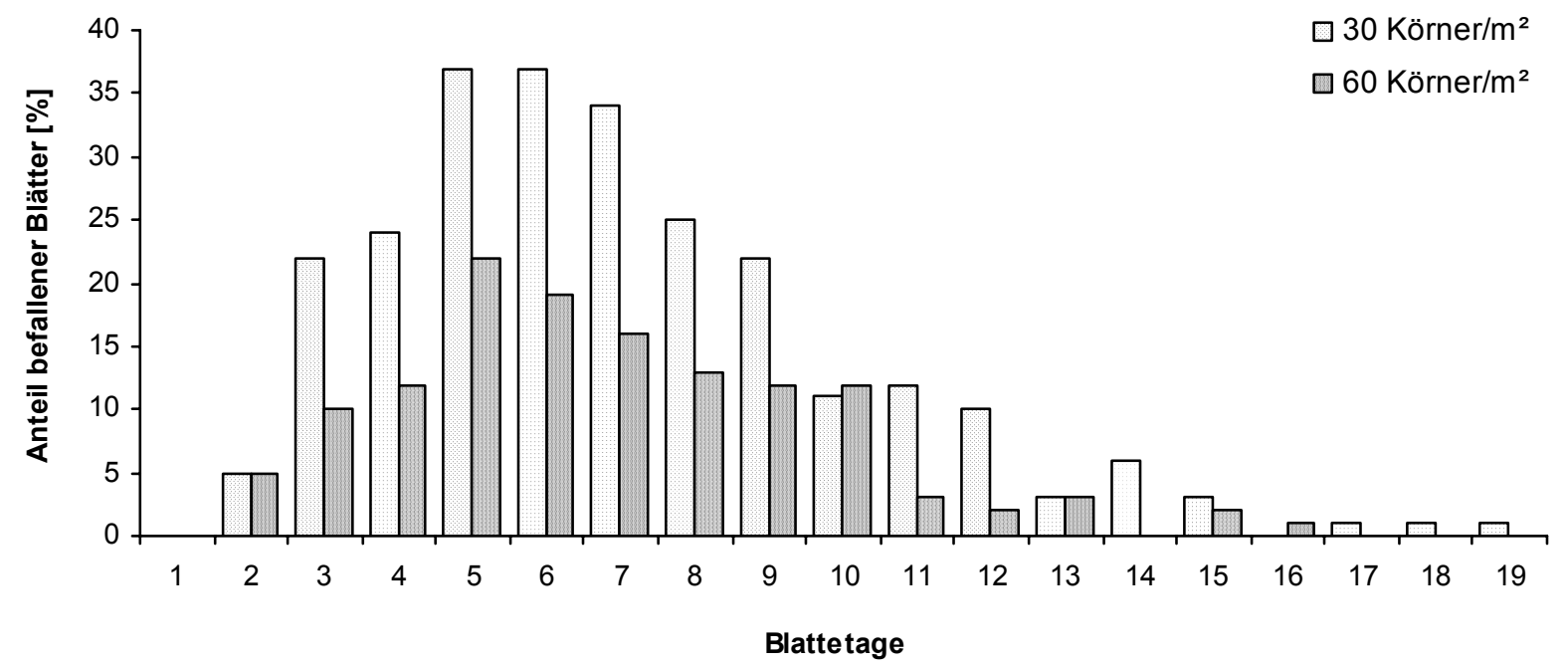

Abb. 41: Mittlere Befallshäufigkeit von C. pallidactylus in verschiedenen Blattetagen der Pflanzen unterschiedlicher Saatstärken im April 2002 am Standort Otto-Hahn-Straße 


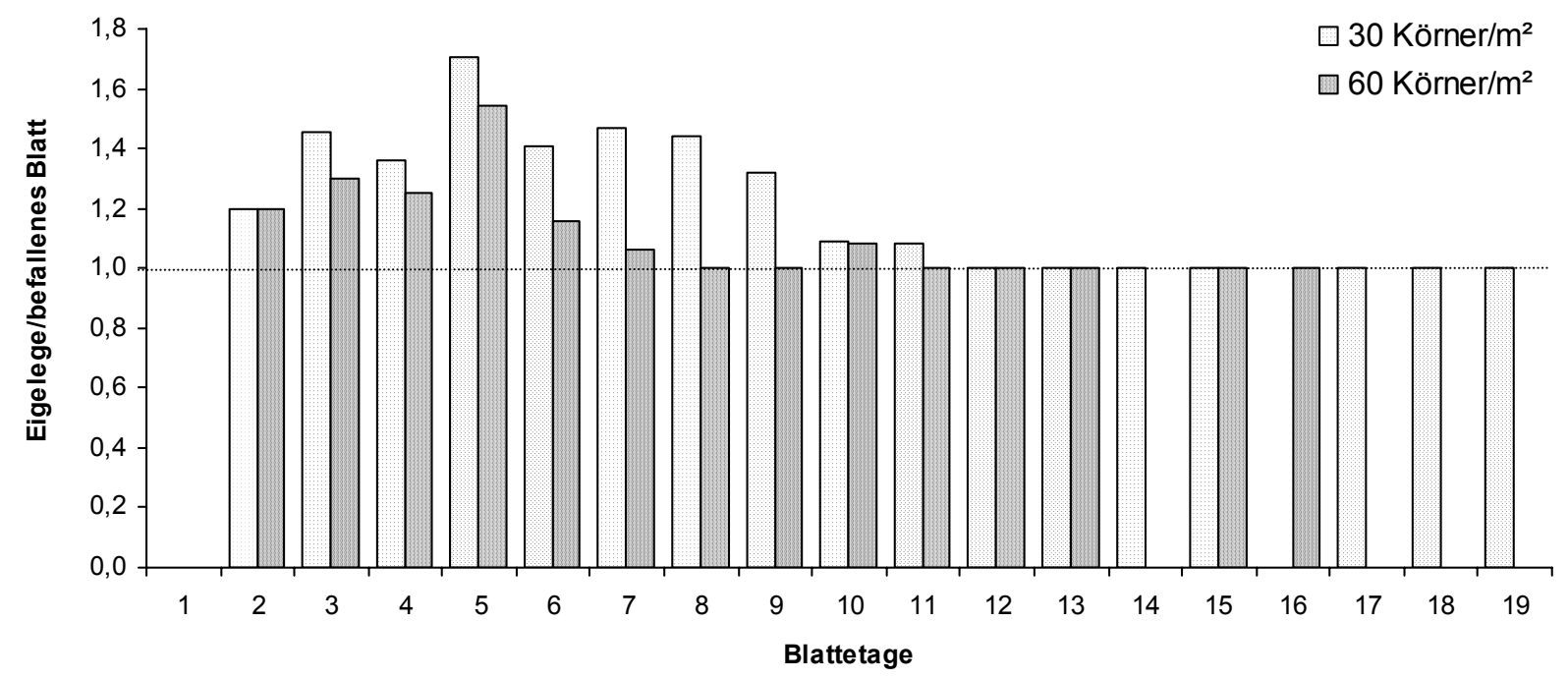

Abb. 42: Mittlere Anzahl der Eigelege/Blattetage von C. pallidactylus in befallenen Blättern der Pflanzen unterschiedlicher Saatstärken im April 2002 am Standort Otto-HahnStraße

\subsubsection{Verteilung der Eigelege von $C$. pallidactylus auf das basale, mittlere und apikale}

\section{Drittel der Blätter}

Bei der getrennten Erfassung der Eigelege in drei gleich großen Blattabschnitten (basales, mittleres und apikales Drittel) im April 2002 zeigte sich, dass die Eiablage von C. pallidactylus in verschiedene Blattbereiche durch die Saatstärke nicht signifikant beeinflusst wurde (ANOVA, Covariate(s)-Test, Saatstärke als Covariate und Blattabschnitte als Variate, $F=0,025$, $p=0,876$ bzw. $F=23,385, p=0,000)$. Bei dem Vergleich der drei Blattabschnitte einzelner Saatstärken ergab sich für die Saatstärke $60 \mathrm{Kö.} / \mathrm{m}^{2}$ eine signifikante Bevorzugung des mittleren Blattbereichs gegenüber dem basalen und dem apikalen Bereich (Abb. 43). Bei der Saatstärke $30 \mathrm{Kö.} / \mathrm{m}^{2}$ war lediglich ein signifikanter Unterschied zwischen dem mittleren und dem basalen Blattabschnitt festzustellen. Darüber hinaus fiel bei dieser Saatstärke die Häufigkeit der Eiablagestellen in Richtung Blattspitze weniger stark ab als bei der Saatstärke $60 \mathrm{Kö.} / \mathrm{m}^{2}$. 


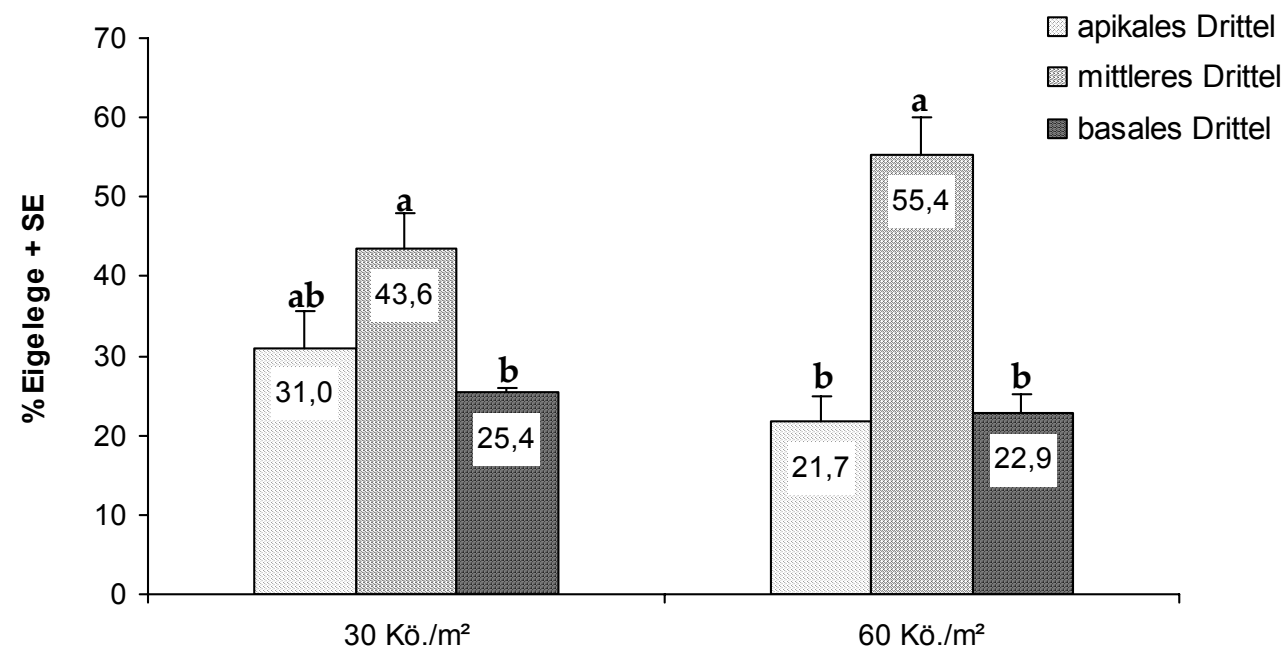

Abb. 43: Verteilung der Eigelege von C. pallidactylus in verschiedenen Blattbereichen einzelner Saatstärken im April 2002 am Standort Otto-Hahn-Straße (Unterschiedliche Buchstaben indizieren signifikante Unterschiede innerhalb der Säulengruppe, ARCSIN-Transformation, Kruskal-Wallis-Test, $\mathrm{p}<0,05$ )

\subsubsection{Häufigkeitsverteilung unterschiedlich großer Eigelege von $C$. pallidactylus an den Blättern der Haupttriebe}

Zur Feststellung der Eigelegegröße von C. pallidactylus an den Blättern der Haupttriebe wurden die Daten des Apriltermins aus dem Jahr 2002 verwendet, weil nur in diesem Jahr eine ausreichend hohe Anzahl der Eigelege von C. pallidactylus an den Blättern der Haupttriebe gegeben war. Der relativ hohe Anteil der Eiablagestellen mit Wundkallusbildung im April 2000 und eine insgesamt sehr geringe Eiablage im April 2001 ließen in diesen Jahren keine weitergehende Auswertung zu. Es wurden nur die Eigelege berücksichtigt, deren Eier noch nicht durch Wundkallus der Pflanze im Bereich der Eiablageorte zerquetscht oder herausgedrückt worden waren.

In Abbildung 44 ist die Häufigkeitsverteilung der Eigelege in Klassen mit 1 bis 11 Eiern pro Gelege bei zwei unterschiedlichen Saatstärken dargestellt. Die beiden Häufigkeitsverteilungen lassen sich mit Hilfe der Regressionsanalyse beschreiben. Während sich bei der Saatstärke 60 Kö. $/ \mathrm{m}^{2}$ ein größerer Anteil von Eigelegen im Bereich von 4-5 Eiern/Gelege findet, ist bei der Saatstärke $30 \mathrm{Kö.} / \mathrm{m}^{2}$ ein höherer Prozentsatz von kleineren Eigelegen mit 3 und weniger Eiern festzustellen. Dies wird auch anhand des Mittelwertes deutlich: Bei der Saatstärke $60 \mathrm{Kö.} / \mathrm{m}^{2}$ waren 3,9 Eier/Gelege vorhanden, bei der Saatstärke $30 \mathrm{Kö.} / \mathrm{m}^{2}$ 3,4 Eier/Gelege. 


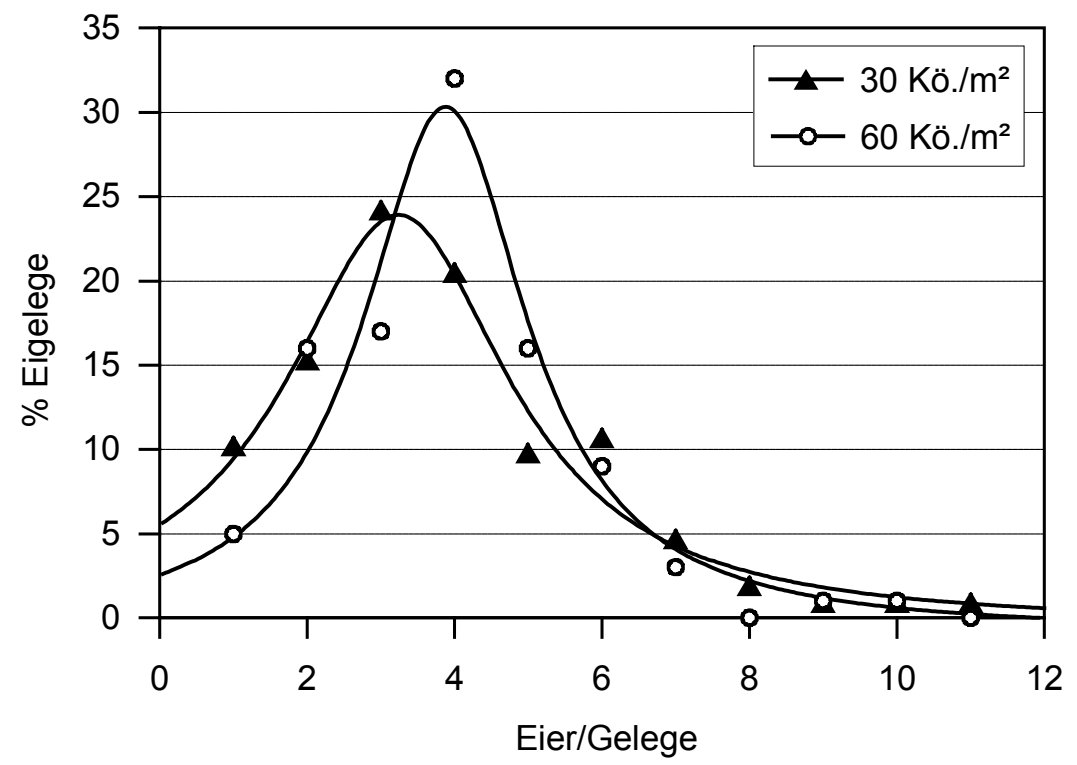

\begin{tabular}{ccccc}
\hline Variante & $\mathrm{n}$ & Gleichung & $\mathrm{r}^{2}$ & $\mathrm{p}$ \\
\hline $30 \mathrm{Kö.} / \mathrm{m}^{2}$ & 11 & $\mathrm{f}(\mathrm{x})=-0,47+\left(2^{*} 141,86 / \mathrm{PI}\right)^{*}\left(3,70 /\left(4^{*}(\mathrm{x}-3,23)^{\wedge} 2+3,70^{\wedge} 2\right)\right)$ & 0,97 & $\leq 0,00004$ \\
$60 \mathrm{Kö.} / \mathrm{m}^{2}$ & 11 & $\mathrm{f}(\mathrm{x})=-0,87+\left(2^{*} 133,02 / \mathrm{PI}\right)^{*}\left(2,72 /\left(4^{*}(\mathrm{x}-3,88)^{\wedge} 2+2,72^{\wedge} 2\right)\right)$ & 0,93 & $\leq 0,00012$ \\
\hline
\end{tabular}

Abb. 44: Häufigkeitsverteilung der Eigelege von $C$. pallidactylus mit verschiedener Anzahl Eier pro Gelege an Blättern der Haupttriebe bei verschiedenen Saatstärken im April 2002 am Standort Otto-Hahn-Straße (Nichtlineare Regression nach Lorentz)

\subsubsection{Verteilung und Größe der Eigelege von $C$. pallidactylus in den Blattstielen, in dem an den Blattstiel angrenzenden Bereich der basalen Blattfiedern und der Blattspreitenbasis sowie in den Verzweigungen der Blattadern}

Bei der Untersuchung der Verteilung der Eigelege von C. pallidactylus in den Blättern der Haupttriebe im April 2002 zeigte sich, dass neben den Blattstielen und -mittelrippen auch der an den Blattstiel angrenzende Bereich der basalen Blattfiedern und der Blattspreitenbasis sowie die Verzweigungen der Blattadern in der Blattspreite mit Eiern belegt werden können. Bei der Blattspreitenbasis erfolgte die Eiablage seitlich sowie auf der Ober- und Unterseite der Blätter, bei den Verzweigungen der Blattadern stets auf der Unterseite der Blätter (Abb. 45). 


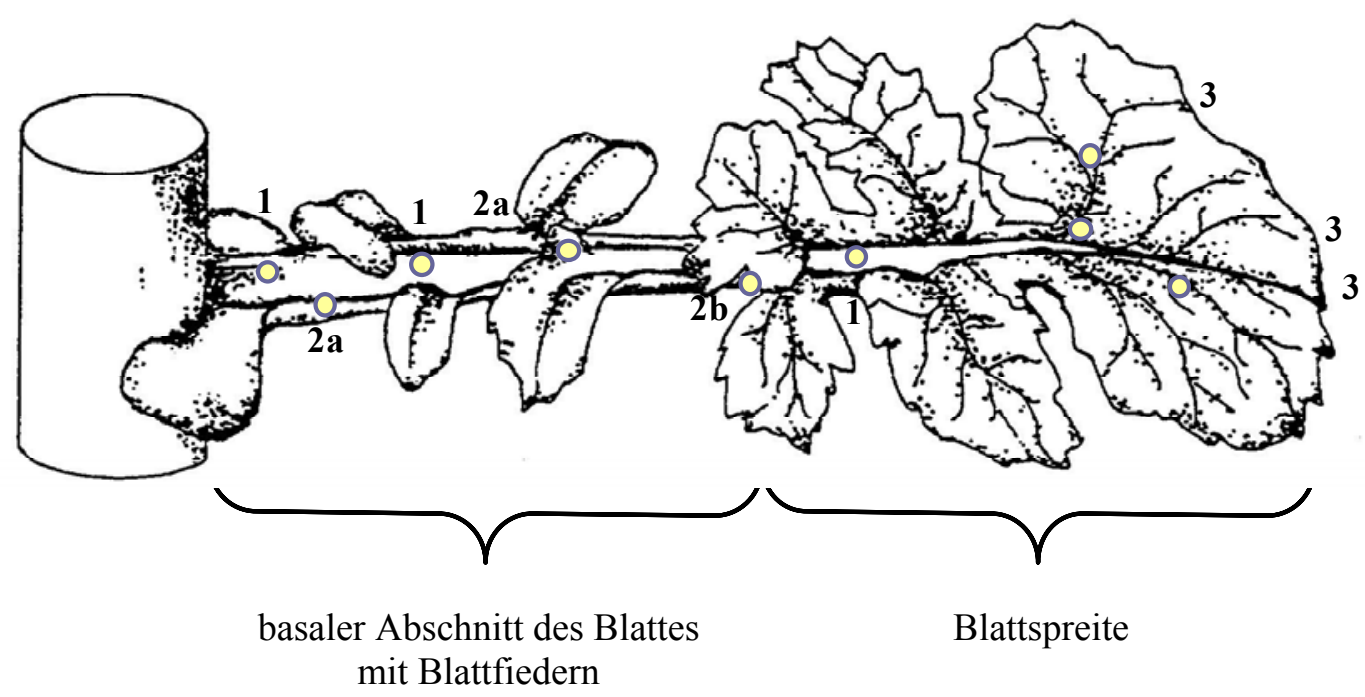

1 Eigelege im Blattstiel und in der Mittelrippe des Blattes

2 Eigelege in dem an den Blattstiel angrenzenden Bereich der basalen Blattfiedern und der Blattspreitenbasis

2a Übergang zwischen dem Blattstiel und den basalen Blattfiedern

2b Übergang zwischen dem Blattstiel und der Blattspreite

3 Verzweigungen der Blattadern

Abb. 45: Positionen der Eigelege im Blattstiel und in der Mittelrippe, in dem an den Blattstiel angrenzenden Bereich der basalen Blattfiedern und der Blattspreitenbasis sowie in den Verzweigungen der Blattadern in den Blättern der Rapspflanzen (verändert nach NISSEN 1997)

Der größte Teil der Eier fand sich im Blattstiel und in der Mittelrippe (Abb. 46). Es wird deutlich, dass sowohl der an den Blattstiel angrenzende Bereich der basalen Blattfiedern und der Blattspreitenbasis als auch die Verzweigungen der Blattadern bei der Saatstärke $30 \mathrm{Kö.} / \mathrm{m}^{2}$ signifikant häufiger mit Eiern belegt wurden als bei der Saatstärke $60 \mathrm{Kö.} / \mathrm{m}^{2}$ (Abb. 46). Der Anteil der Eigelege in den Blattstielen verhielt sich konträr dazu.

In Abbildung 47 ist die mittlere Anzahl der Eier pro Eigelege von C. pallidactylus in den oben genannten Bereichen des Blattes dargestellt. Dabei wurden nur die Eigelege berücksichtigt, die noch nicht durch die Wundkallusbildung herausgedrückt worden waren. Die mittlere Größe der Eigelege wies weder im Blattstiel (Minimum: 1 Ei, Maximum: 11 Eier) noch in dem an den Blattstiel angrenzende Bereich der basalen Blattfiedern und der Blattspreitenbasis (Minimum: 1 Ei, Maximum: 9 Eier) gesicherte Unterschiede zwischen den beiden Saatstärken auf. Der geringfügige Anteil der Eigelege in den Verzweigungen der Blattadern (Minimum: 1 Ei, Maximum: 7 Eier) ließ bei der Saatstärke $60 \mathrm{Kö.} / \mathrm{m}^{2}$ keine weitergehende Auswertung zu. 


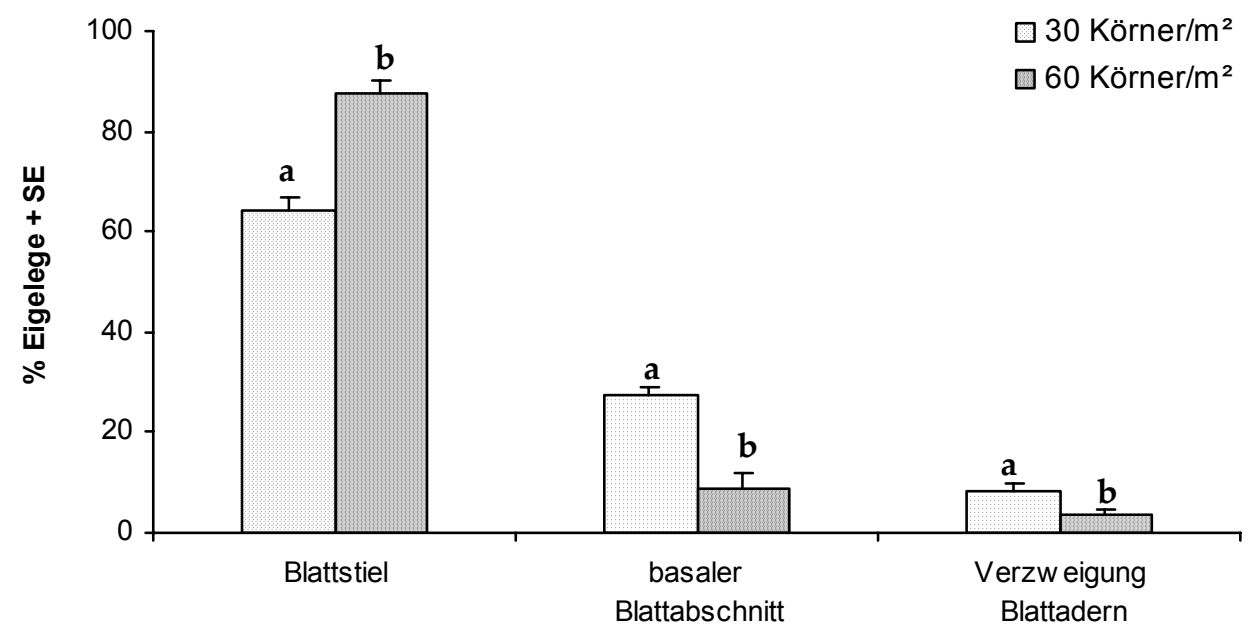

Abb. 46: Verteilung der Eigelege von $C$. pallidactylus auf Blattstiele, an den Blattstiel angrenzende Bereiche der basalen Blattfiedern und der Blattspreitenbasis (basaler Blattabschnitt) sowie Verzweigungen der Blattadern in Blättern der Haupttriebe verschiedener Saatstärken im April 2002 am Standort Otto-Hahn-Straße (Unterschiedliche Buchstaben indizieren signifikante Unterschiede zwischen den Saatstärken, ARCSIN-Transformation, Mann-Whitney U-Test, $\mathrm{p}<0,05$ )

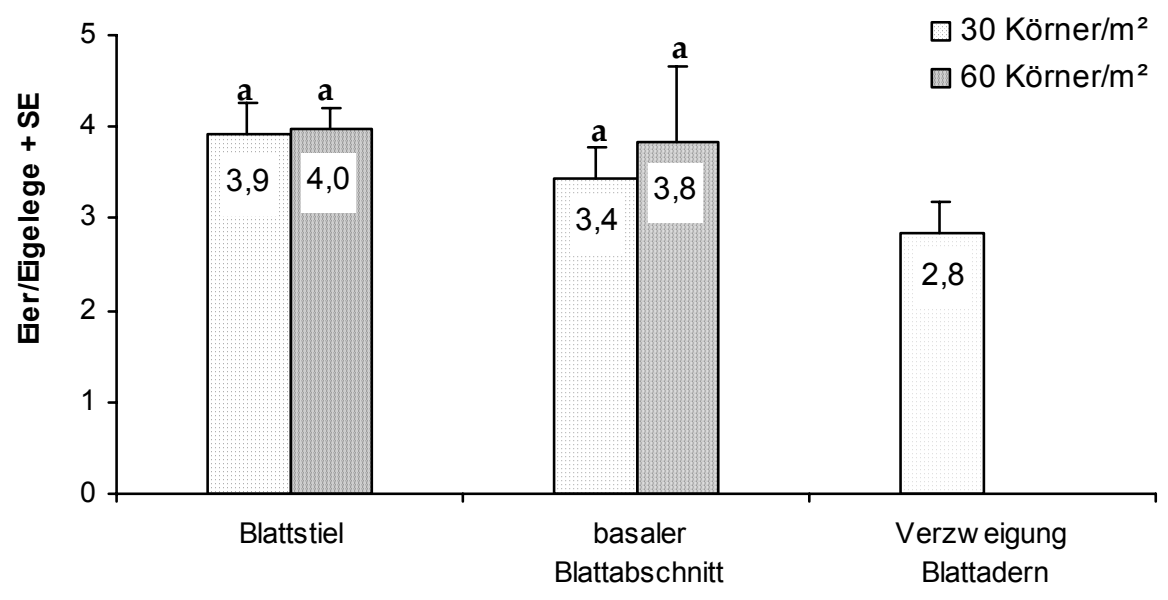

Abb. 47: Mittlere Anzahl der Eier/Eigelege von C. pallidactylus in den Blattstielen, in die an den Blattstiel angrenzenden Bereiche der basalen Blattfiedern und der Blattspreitenbasis (basaler Blattabschnitt) sowie in den Verzweigungen der Blattadern in Blättern verschiedener Saatstärken im April 2002 am Standort Otto-Hahn-Straße (Unterschiedliche Buchstaben indizieren signifikante Unterschiede zwischen den Saatstärken, Mann-Whitney U-Test, $\mathrm{p}<0,05$ ) 


\subsubsection{Verteilung verschiedener Larvenstadien von $C$. napi und $C$. pallidactylus auf Haupt- und Seitentriebe}

\subsubsection{Altersstruktur der Larven von $C$. napi und $C$. pallidactylus}

Die Altersstruktur der Larven von C. napi und C. pallidactylus wurde jeweils im Mai kurz vor Beginn der Abwanderung der Larven von C. napi bestimmt. Während sich im Jahr 2000 in den Pflanzen aller drei Saatstärken lediglich L2- und L3-Larven von C. napi fanden, waren in den folgenden Jahren auch L1-Larven (2001 und 2002) sowie Eier (2002) zu verzeichnen (Abb. 48). Die L3-Larven stellten in allen Jahren den bei weitem größten Anteil. Bei dem Vergleich der verschiedenen Saatvarianten zeigten sich lediglich im Mai 2002 gesicherte Unterschiede im Auftreten der Eier: Bei der Saatstärke $30 \mathrm{Kö.} / \mathrm{m}^{2}$ war der Anteil der Eier von $C$. napi am Gesamtbefall um den Faktor 2 höher als bei der Saatstärke $60 \mathrm{Kö.} / \mathrm{m}^{2}$. Auch die L1und L2-Larven waren bei der Saatstärke $30 \mathrm{Kö.} / \mathrm{m}^{2}$ tendenziell häufiger als bei der Saatstärke $60 \mathrm{Kö.} / \mathrm{m}^{2}$, wie auch im Jahr 2001. Dagegen war der Anteil der L2-Larven in Saatvariante 30 $\mathrm{Kö.} / \mathrm{m}^{2} \mathrm{im}$ Jahr 2000 deutlich geringer als in den Varianten 60 und $90 \mathrm{Kö.} / \mathrm{m}^{2}$.

Bei der Altersstruktur der Larven von C. pallidactylus fanden sich in allen Saatvarianten der drei Versuchsjahre Larven der Entwicklungsstadien L1 bis L3 (Abb. 49). Die Anteile der einzelnen Larvenstadien unterschieden sich nicht signifikant zwischen den einzelnen Varianten. Auffällig war jedoch der hohe Anteil der L1-Larven bei der Saatstärke $30 \mathrm{Kö.} / \mathrm{m}^{2} \mathrm{im}$ Versuchsjahr 2001, der den der beiden höheren Saatvarianten deutlich übertraf. Der Anteil der L3Larven war in dieser Variante entsprechend reduziert. 

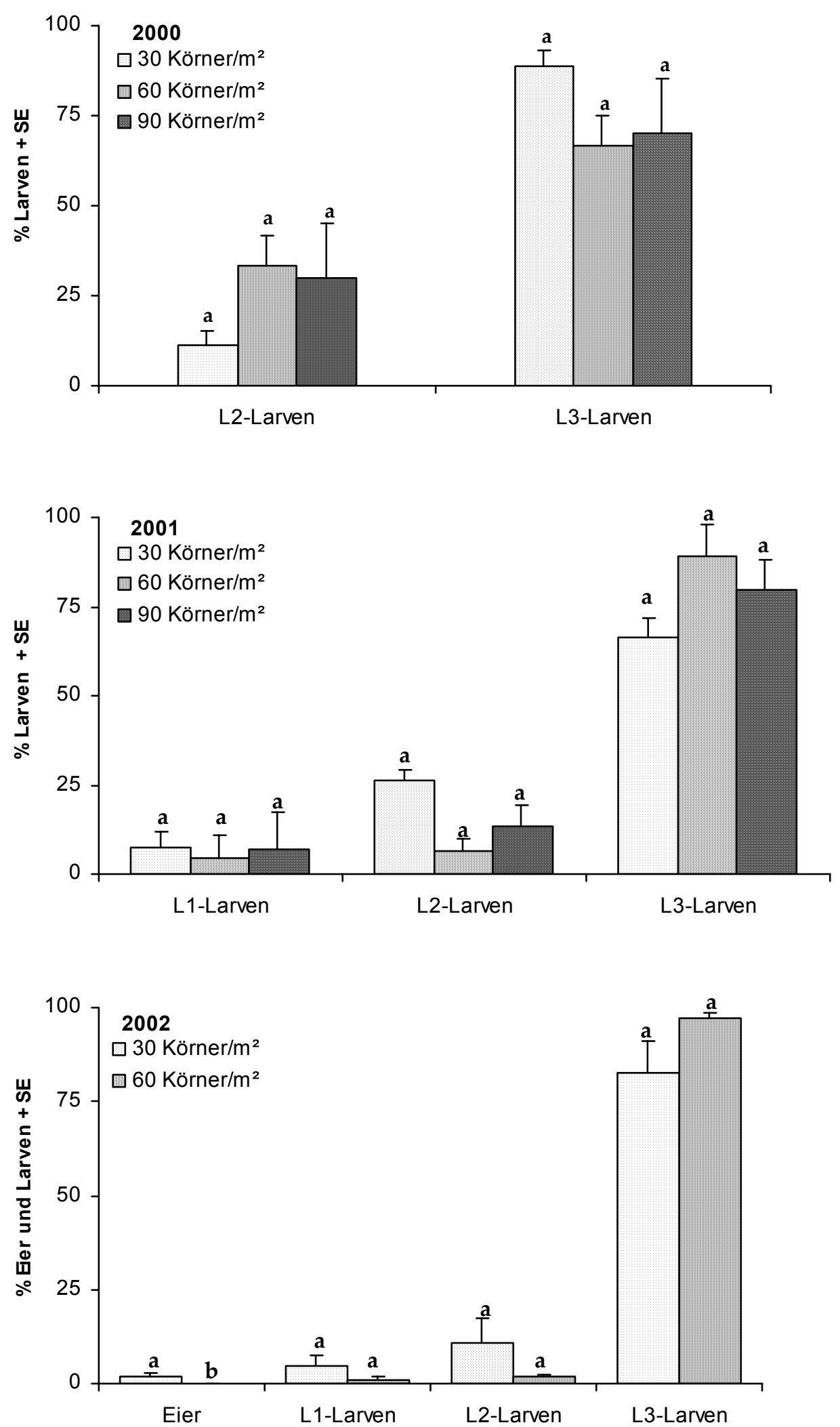

Abb. 48: Altersstruktur von $C$. napi in den Pflanzen unterschiedlicher Saatstärken im Mai 2000 und 2001 am Standort Weendelsbreite und im Mai 2002 am Standort OttoHahn-Straße (Unterschiedliche Buchstaben stellen signifikante Unterschiede zwischen den Saatstärken dar, ARCSIN-Transformation, 2000 und 2001: ANOVA, Tukey-Test, $\mathrm{p}<0,05,2002$ : Mann-Whitney U-Test, $\mathrm{p} \leq 0,05$ ) 

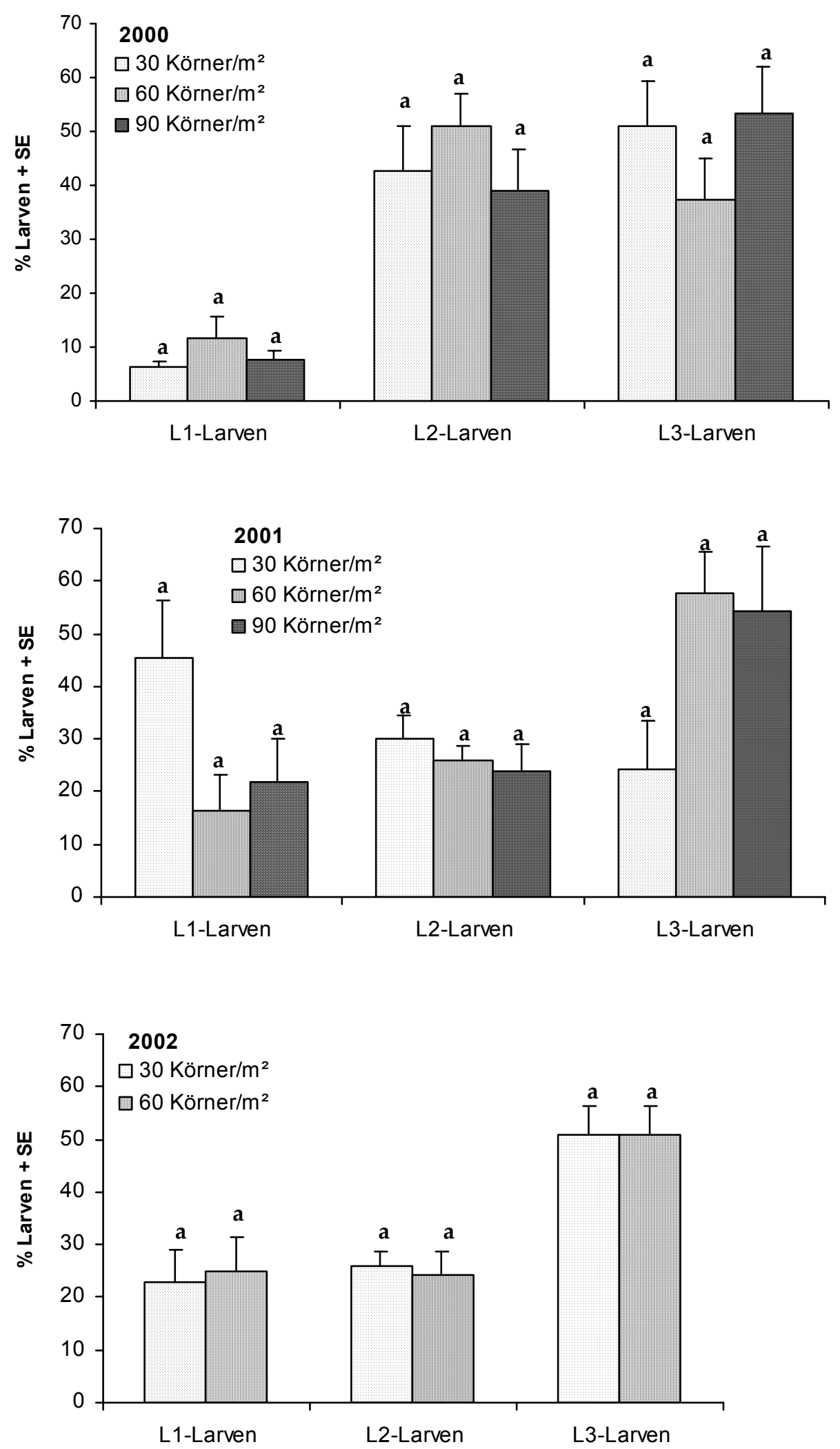

Abb. 49: Altersstruktur von C. pallidactylus in den Pflanzen unterschiedlicher Saatstärken im Mai 2000 und 2001 am Standort Weendelsbreite und im Mai 2002 am Standort Otto-Hahn-Straße (Unterschiedliche Buchstaben stellen signifikante Unterschiede zwischen den Saatstärken dar, ARCSIN-Transformation, 2000 und 2001: ANOVA, Tukey-Test, 2002: Mann-Whitney U-Test, $\mathrm{p}<0,05$ ) 


\subsubsection{Verteilung verschiedener Larvenstadien von $C$. napi auf Haupt- und Seiten- triebe}

Die Verteilung der unterschiedlichen Larvenstadien von C. napi auf die Haupt- und Seitentriebe im Mai der Versuchsjahre 2000 bis 2002 ließ im Befall der Seitentriebe mit den L3-Larven deutliche Differenzen zwischen den Saatstärken erkennen (Abb. 50). Der Anteil der L3Larven in den Seitentrieben war in allen drei Versuchen bei der Saatstärke $30 \mathrm{Kö.} / \mathrm{m}^{2}$ am höchsten und fiel mit zunehmender Saatstärke ab. Die deutlichsten Unterschiede zwischen den Saatvarianten traten in den Versuchen 2000 und 2002 auf. Der Anteil der L3-Larven in den Seitentrieben in der Variante $30 \mathrm{Kö.} / \mathrm{m}^{2}$ unterschied sich in beiden Versuchen signifikant von dem der übrigen Varianten. Im Versuch 2001 waren die Unterschiede geringer und konnten, bedingt durch die große Streuung der Einzelwerte, statistisch nicht abgesichert werden. Darüber hinaus war der Anteil der L3-Larven in den Seitentrieben im Versuch 2001 bei den Saatstärken 60 und $90 \mathrm{Kö.} / \mathrm{m}^{2}$ deutlich höher als bei den Versuchen 2000 und 2002.

Der Anteil der L3-Larven in den Haupttrieben stieg dagegen in den Versuchen 2001 und 2002 mit zunehmender Saatstärke an. Im Versuch 2001 war der Anteil der L3-Larven in den Haupttrieben bei der Saatstärke $30 \mathrm{Kö.} / \mathrm{m}^{2}$ deutlich geringer als bei den Saatstärken 60 und 90 Kö./m², die ähnliche Werte aufwiesen. Im Versuch 2002 unterschieden sich die beiden Saatstärken 30 und $60 \mathrm{Kö.} / \mathrm{m}^{2}$ signifikant voneinander.

Bei dem Anteil der jüngeren Larvenstadien L1 und L2 in den Seitentrieben zeigten sich nur geringe, nicht statistisch sicherbare Abweichungen zwischen den Saatstärken. Der nur im Versuch 2002 zu beobachtende Befall der Seitentriebe mit den Eiern von C. napi war bei der Saatstärke $30 \mathrm{Kö.} / \mathrm{m}^{2}$ signifikant höher als bei der Saatstärke $60 \mathrm{Kö.} / \mathrm{m}^{2}$.

In den Haupttrieben war nur bei den L2-Larven im Versuch 2000 eine deutliche Abstufung zwischen den Saatstärken zu verzeichnen: Der Anteil der L2-Larven in den Haupttrieben stieg mit zunehmender Saatstärke an. 

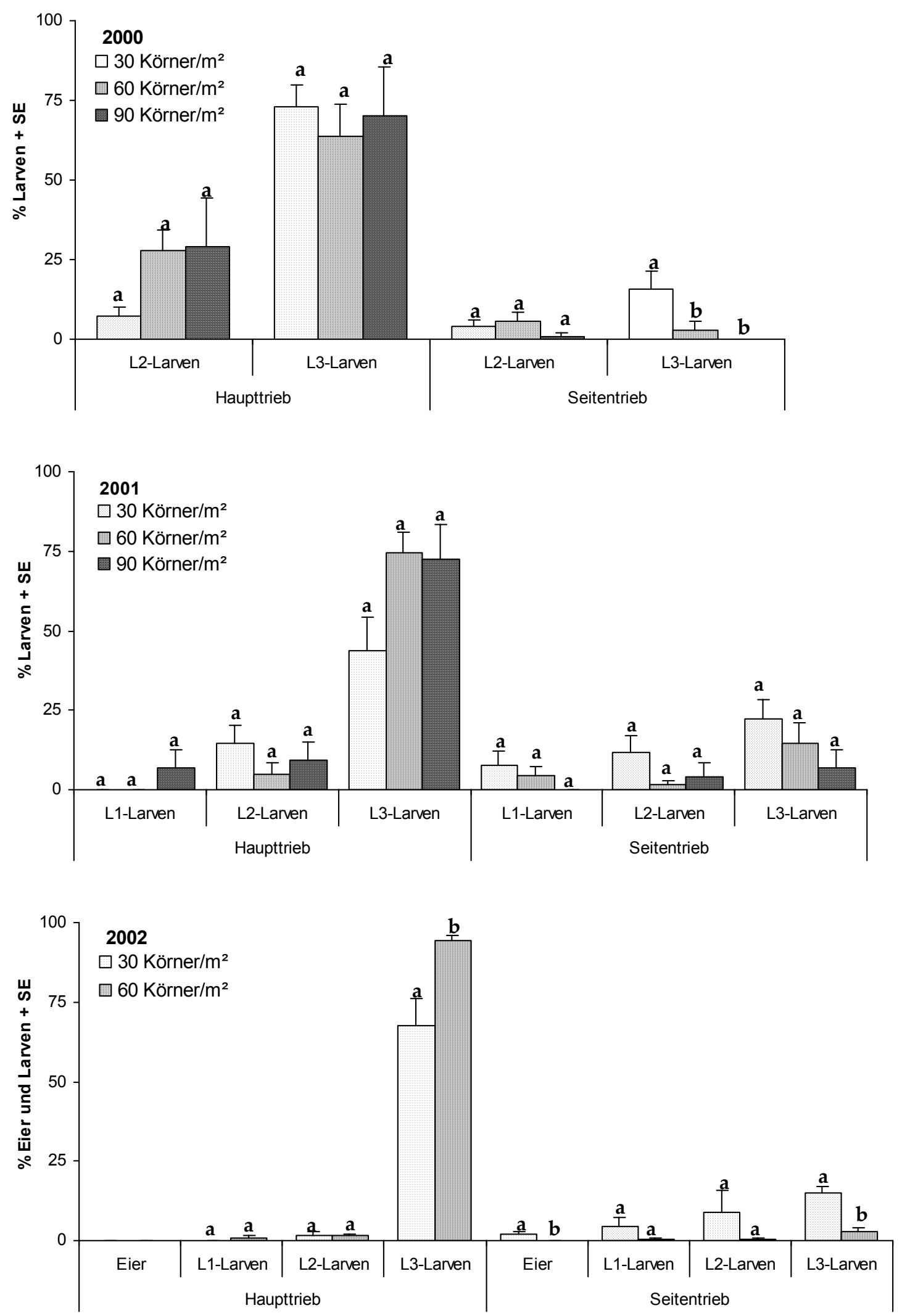

Abb. 50: Verteilung unterschiedlicher Präimaginalstadien von C. napi auf die Haupt- und Seitentriebe der Pflanzen verschiedener Saatstärken im Mai 2000 und 2001 am Standort Weendelsbreite und im Mai 2002 am Standort Otto-Hahn-Straße (Verschiedene Buchstaben indizieren signifikante Unterschiede zwischen den Saatstärken, ARCSIN-Transformation, 2000 und 2001: ANOVA, Tukey-Test, $p<0,05$, 2002: Mann-Whitney U-Test, $p \leq 0,05$ ) 


\subsubsection{Verteilung verschiedener Larvenstadien von $C$. pallidactylus auf Haupt- und Seitentriebe}

Bei der Verteilung der unterschiedlichen Larvenstadien von C. pallidactylus auf die Hauptund Seitentriebe im Mai der Versuchsjahre 2000 bis 2002 wird deutlich, dass die Seitentriebe im Versuch 2000 weniger stark mit Eiern belegt wurden als in den Versuchen 2001 und 2002 (Abb. 51). Auffällig ist zudem der relativ hohe Anteil der L1-Larven in den Seitentrieben in den Versuchen 2001 und 2002 gegenüber dem Versuch 2000. Der Anteil der L2-Larven in den Haupttrieben verhielt sich entgegengesetzt dazu.

Im Versuch 2000 zeigte sich nur für den Befall der Seitentriebe mit den L2- und L3-Larven eine deutliche Abstufung zwischen den Saatstärken: Der Anteil der L2- und L3-Larven war bei der Saatstärke $30 \mathrm{Kö.} / \mathrm{m}^{2}$ signifikant höher als bei der Saatstärke $90 \mathrm{Kö.} / \mathrm{m}^{2}$. Im Versuch 2001 waren sowohl für die Haupt- als auch für die Seitentriebe deutliche Unterschiede zwischen den Saatstärken zu verzeichnen. Der Anteil der L1- und L2-Larven in den Seitentrieben war bei der Saatstärke $30 \mathrm{Kö.} / \mathrm{m}^{2}$ am höchsten und nahm mit ansteigender Saatstärke ab. Die Saatstärke $60 \mathrm{Kö.} / \mathrm{m}^{2}$ führte nur bei den L2-Larven nicht zu signifikanten Unterschieden. Die Haupttriebe der Saatstärken 60 und $90 \mathrm{Kö.} / \mathrm{m}^{2}$ zeigten einen 3-fach höheren Anteil an L3Larven als die Haupttriebe der Saatstärke $30 \mathrm{Kö.} / \mathrm{m}^{2}$. Im Versuch 2002 bot sich ein ähnliches Bild wie 2001: Ein signifikanter Unterschied zwischen den Saatstärken war jedoch nur für den Befall der Seitentriebe mit den L3-Larven zu beobachten. 

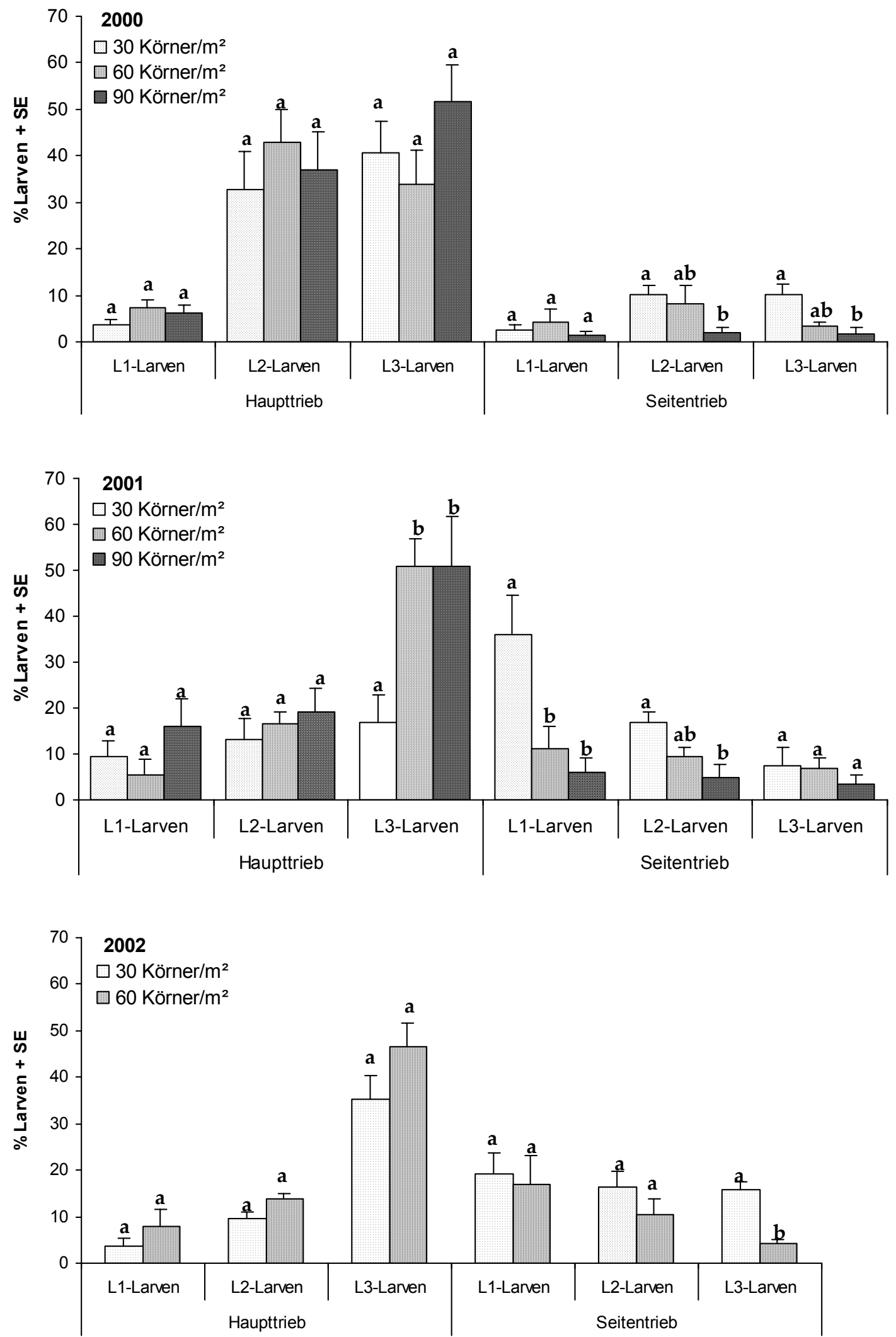

Abb. 51: Verteilung unterschiedlicher Larvenstadien von C. pallidactylus auf die Haupt- und Seitentriebe der Pflanzen verschiedener Saatstärken im Mai 2000 und 2001 am Standort Weendelsbreite und im Mai 2002 am Standort Otto-Hahn-Straße (Verschiedene Buchstaben indizieren signifikante Unterschiede zwischen den Saatstärken, ARCSIN-Transformation, 2000 und 2001: ANOVA, Tukey-Test, 2002: MannWhitney U-Test, $\mathrm{p}<0,05$ ) 


\subsubsection{Einfluss des Stängelbasisdurchmessers der Rapspflanzen auf die Eiablage von}

\section{C. napi und C. pallidactylus}

Um zu prüfen, ob die Weibchen von $C$. napi und $C$. pallidactylus eine Eiablagepräferenz für Pflanzen einer bestimmten Stängeldicke besitzen, wurden am 2. April 2002 in einer gesonderten Pflanzenprobe zu einem frühen Zeitpunkt der Eiablage Pflanzen mit großen und kleinen Stängeldurchmessern aus der Mantelsaat untersucht. In Tabelle 42 sind die Wachstumsparameter der Rapspflanzen mit dicken und dünnen Stängeln wiedergegeben. Die Anzahl der Blätter und Seitentriebe sowie die Länge der Pflanzen waren bei den Pflanzen mit dicken Stängeln signifikant höher als bei den schwächeren Pflanzen. Darüber hinaus hatten die Pflanzen mit dicken Stängeln bereits ausgebildete Seitentriebe mit sichtbarem Längen- und Dickenwachstum. Bei den Pflanzen mit dünnen Stängeln hingegen war der bei weitem größte Anteil der Seitentriebanlagen noch im Knospenstadium.

Tab. 42: Mittelwerte der Wachstumsparameter ( \pm SD) der Rapspflanzen mit dicken und dünnen Stängeln (BBCH 50-52) im April 2002 am Standort Otto-Hahn-Straße

\begin{tabular}{|c|c|c|c|c|c|c|}
\hline Variante & $\begin{array}{l}\text { Sprossdurch- } \\
\text { messer am } \\
\text { Wurzelhals } \\
{[\mathrm{mm}]}\end{array}$ & $\begin{array}{l}\text { Anzahl } \\
\text { Blätter }\end{array}$ & $\begin{array}{c}\text { Anzahl } \\
\text { Seitentriebe }\end{array}$ & $\begin{array}{c}\text { Spross- } \\
\text { länge } \\
{[\mathrm{cm}]}\end{array}$ & $\begin{array}{c}\text { Länge } \\
\text { der } \\
\text { Seitentriebe } \\
{[\mathrm{cm}]}\end{array}$ & $\begin{array}{c}\text { Durch- } \\
\text { messer der } \\
\text { Seitentriebe } \\
{[\mathrm{mm}]}\end{array}$ \\
\hline & $\mathrm{X}_{\mathrm{m}} \pm \mathrm{SD}$ & $\mathrm{X}_{\mathrm{m}} \pm \mathrm{SD}$ & $\mathrm{X}_{\mathrm{m}} \pm \mathrm{SD}$ & $\mathrm{X}_{\mathrm{m}} \pm \mathrm{SD}$ & $\mathrm{X}_{\mathrm{m}} \pm \mathrm{SD}$ & $\mathrm{X}_{\mathrm{m}} \pm \mathrm{SD}$ \\
\hline dick & $17,3 \pm 2,0$ a & $23,4 \pm 1,8 a$ & $10,1 \pm 6,5 a$ & $28,0 \pm 3,9 a$ & $1,5 \pm 0,2$ & $2,0 \pm 0,2$ \\
\hline dünn & $9,2 \pm 1,1 b$ & $16,4 \pm 1,9 b$ & $1,1 \pm 2,1 b$ & $23,9 \pm 3,0 b$ & - & - \\
\hline
\end{tabular}

Verschiedene Buchstaben indizieren signifikante Unterschiede zwischen den Saatstärken, ANOVA, LSD-Test, $p \leq 0,0001$

In den Pflanzen mit den dicken Stängeln war die Dichte der Eigelege bei C. napi und C. pallidactylus mit 8,4 bzw. 2,3 Eiablagestellen/Pflanze jeweils signifikant höher als in den Pflanzen mit den dünnen Stängeln (2,0 bzw. 1,1 Eiablagestellen/Pflanze) (Abb. 52).

Eine Eiablage von C. napi in die Seitentriebe war lediglich bei den Pflanzen mit dicken Stängeln möglich. Der Anteil belegter Seitentriebe betrug 10,4 \%.

Die Weibchen von C. pallidactylus legten bei den dicken und dünnen Pflanzen den bei weitem größten Teil der Eier in die Blätter der Haupttriebe ab (vgl. Kap. 3.3.9.3). Nur bei den dicken Pflanzen fanden sich darüber hinaus 1,2 \% der Eigelege in den Blättern der Seitentriebe. Bei den dünnen Pflanzen wurden neben den Blättern der Haupttriebe auch die Haupttriebe selbst mit Eigelegen versehen. Hier waren 2,2 \% der Eigelege nachzuweisen. 


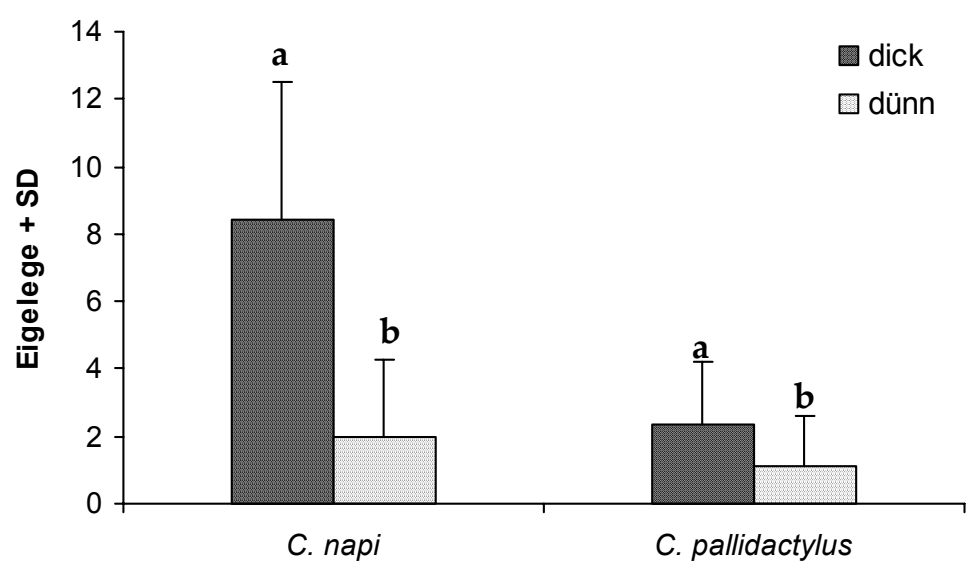

Abb. 52: Mittlere Anzahl Eigelege/Pflanze von C. napi und C. pallidactylus in Rapspflanzen mit dicken und dünnen Stängeln (BBCH 50-52) im April 2002 am Standort OttoHahn-Straße (Verschiedene Buchstaben indizieren signifikante Unterschiede zwischen den dicken und dünnen Pflanzen, $C$. napi: ANOVA, LSD-Test, $\mathrm{p} \leq 0,001, C$. pallidactylus: Two Sample KS-Test, $\mathrm{p} \leq 0,05$ )

In Abbildung 53 ist die Beziehung zwischen dem Durchmesser des Rapsstängels an der Basis und der Anzahl Eiablagestellen/Pflanze von C. napi und C. pallidactylus dargestellt. Es zeigte sich für beide Ceutorhynchus-Arten ein hochsignifikanter Zusammenhang zwischen den beiden Parametern. Mit einer Zunahme des Stängeldurchmessers kam es zu einer drastischen Zunahme der Anzahl Eiablagestellen pro Pflanze. Bezogen auf den Bestimmtheitsmaß war diese Beziehung bei $C$. pallidactylus allerdings deutlich schwächer ausgeprägt als bei $C$. napi.
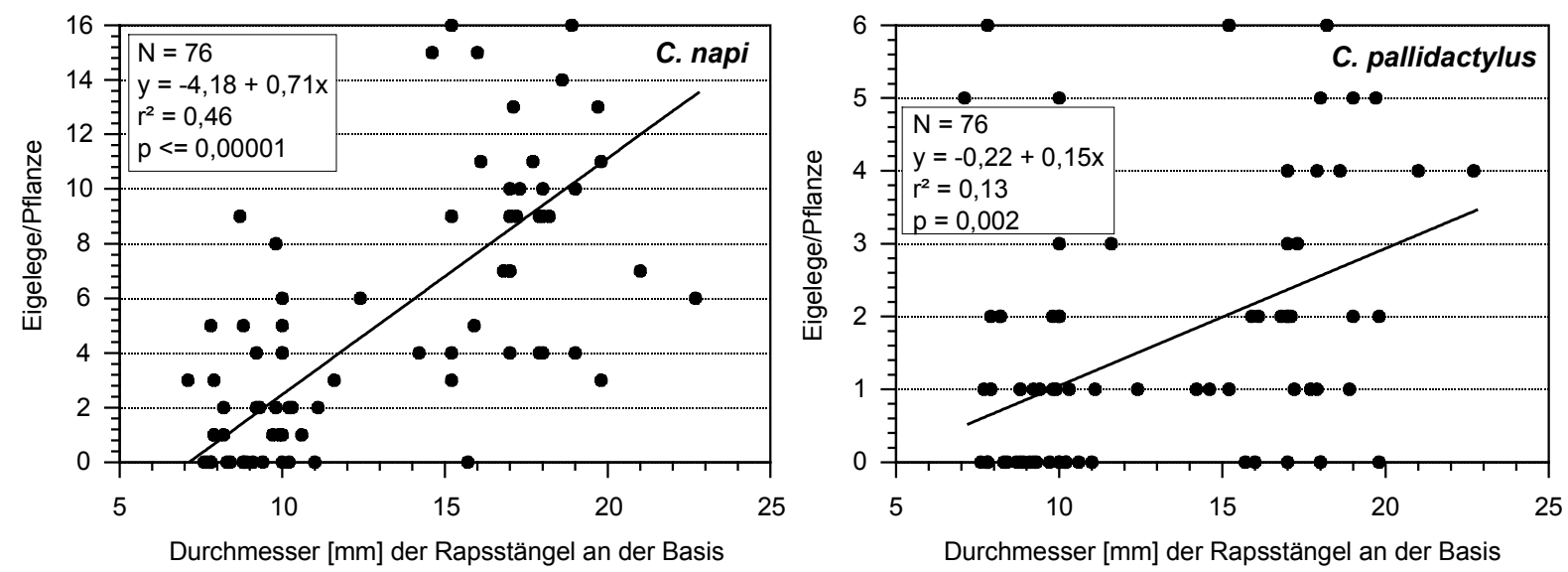

Abb. 53: Anzahl Eigelege/Pflanze von $C$. napi und C. pallidactylus in Abhängigkeit vom Durchmesser der Rapsstängel an der Basis im April 2002 (BBCH 50-52) am Standort Otto-Hahn-Straße 


\subsection{Kornertrag in Abhängigkeit von Pflanzendichte und -architektur}

\subsubsection{Kornertrag}

Die in allen drei Anbaujahren (1999/2000 bis 2001/2002) ermittelten Flächenerträge wiesen sowohl in den Insektizid-behandelten Parzellen (Versuche 1999/00 bis 2001/02) als auch in den unbehandelten Parzellen (Versuche 2000/01 und 2001/02) nur geringe Unterschiede zwischen den Saatvarianten auf. Sie ließen keinen Einfluss der Saatstärke erkennen (Tab. 43). Bei der Liniensorte Mohican im Versuch 1999/00 war das mittlere Ertragsniveau mit 33,1 dt/ha relativ gering. Bei der Hybridsorte Artus im Versuch 2000/01 lag es mit 40,5 dt/ha in den behandelten Parzellen deutlich höher. Im folgenden Anbaujahr 2001/02 erreichte diese Hybridsorte jedoch mit durchschnittlich 30,0 dt/ha den geringsten Ernteertrag im gesamten Untersuchungszeitraum. Ein signifikanter Einfluss der Insektizidbehandlung auf den Ertrag war nur bei der Aussaatvariante $60 \mathrm{Kö.} / \mathrm{m}^{2}$ festzustellen: Die behandelte Saatvariante $60 \mathrm{Kö.} / \mathrm{m}^{2}$ brachte in den Versuchen 2000/01 und 2001/02 einen signifikanten Mehrertrag von 2,1 dt/ha bzw. 3,1 dt/ha. Der Ertragsunterschied zwischen der behandelten und unbehandelten Saatvariante $30 \mathrm{Kö.} / \mathrm{m}^{2}$ im Versuch 2001/02 war nicht absicherbar.

Der Kornertrag/Pflanze nahm sowohl in den behandelten als auch in den unbehandelten Parzellen mit abnehmender Saatstärke signifikant zu (Tab. 44). Die Sorteneigenschaften der Hybridsorte, gekoppelt mit optimalen Aussaat- und Wachstumsbedingungen, führten zu dem im Anbaujahr 2000/01 ermittelten höheren Ertragsniveau pro Pflanze. Die Insektizidbehandlung hatte in den Versuchen 2000/01 und 2001/02 keinen Einfluss auf den Ertrag/Pflanze.

Bei dem Tausendkorngewicht zeigten sich in allen Untersuchungsjahren nur geringe, nicht statistisch sicherbare Unterschiede zwischen den Saatvarianten (Tab. 45). Die Insektizidapplikation hatte keine signifikanten Auswirkungen auf das TKG.

Tab. 43: Mittlerer Kornertrag/ha [dt/ha] ( \pm SE) in Abhängigkeit von der Saatstärke in den Anbaujahren 1999/00 und 2000/01 am Standort Weendelsbreite und im Anbaujahr 2001/02 am Standort Otto-Hahn-Straße

\begin{tabular}{lccccc}
\hline Saatstärke & $1999 / 00$ & \multicolumn{2}{c}{$2000 / 01$} & \multicolumn{2}{c}{$2001 / 02$} \\
& behandelt & behandelt & unbehandelt & behandelt & unbehandelt \\
& $X_{\mathrm{m}} \pm \mathrm{SE}$ & $\mathrm{X}_{\mathrm{m}} \pm \mathrm{SE}$ & $\mathrm{X}_{\mathrm{m}} \pm \mathrm{SE}$ & $\mathrm{X}_{\mathrm{m}} \pm \mathrm{SE}$ & $\mathrm{X}_{\mathrm{m}} \pm \mathrm{SE}$ \\
\hline $30 \mathrm{Körner} / \mathrm{m}^{2}$ & $31,6 \pm 1,2$ a & $39,4 \pm 0,3$ a A & $38,9 \pm 0,7$ a $\mathrm{A}$ & $31,1 \pm 0,9$ a A & $26,2 \pm 2,1$ a $\mathrm{A}$ \\
$60 \mathrm{Körner} / \mathrm{m}^{2}$ & $33,8 \pm 0,8$ a & $41,2 \pm 0,6$ a A & $39,1 \pm 0,3$ a B & $28,9 \pm 0,8$ a A & $25,7 \pm 1,1$ a B \\
$90 \mathrm{Körner} / \mathrm{m}^{2}$ & $33,7 \pm 1,4$ a & $41,0 \pm 0,7$ a A & $39,5 \pm 0,8$ a A & - & - \\
\hline
\end{tabular}

Unterschiedliche Klein- und Großbuchstaben indizieren signifikante Unterschiede zwischen den Saatstärken bzw. Behandlungsvarianten, 1999/00 und 2000/01: ANOVA, Tukey-Test, 2001/02: Mann-Whitney U-Test, $p<0,05$ 
Tab. 44: Mittlerer Kornertrag/Pflanze [g/Pflanze] $( \pm \mathrm{SE})$ in Abhängigkeit von der Saatstärke in den Anbaujahren 1999/00 und 2000/01 am Standort Weendelsbreite und im Anbaujahr 2001/02 am Standort Otto-Hahn-Straße

\begin{tabular}{|c|c|c|c|c|c|}
\hline \multirow[t]{2}{*}{ Saatstärke } & \multirow{2}{*}{$\begin{array}{c}1999 / 00 \\
\text { behandelt } \\
\mathrm{X}_{\mathrm{m}} \pm \mathrm{SE}\end{array}$} & \multicolumn{2}{|c|}{$2000 / 01$} & \multicolumn{2}{|c|}{$2001 / 02$} \\
\hline & & $\begin{array}{l}\text { behandelt } \\
X_{m} \pm S E\end{array}$ & $\begin{array}{l}\text { unbehandelt } \\
X_{m} \pm S E\end{array}$ & $\begin{array}{l}\text { behandelt } \\
X_{m} \pm S E\end{array}$ & $\begin{array}{l}\text { unbehandelt } \\
X_{m} \pm S E\end{array}$ \\
\hline 30 Körner/m² & $8,5 \pm 0,3 a$ & $13,5 \pm 1,1$ a $A$ & $14,1 \pm 0,6$ a $A$ & $13,0 \pm 0,6$ a $A$ & $10,6 \pm 1,2$ a $A$ \\
\hline 60 Körner/m² & $5,1 \pm 0,1 b$ & $7,7 \pm 0,3$ b $A$ & $8,4 \pm 0,3$ b A & $5,9 \pm 0,2 b A$ & $5,2 \pm 0,3$ b $A$ \\
\hline 90 Körner/m² & $4,1 \pm 0,2 \mathrm{c}$ & $5,4 \pm 0,2 b \mathrm{~b}$ & $5,7 \pm 0,2 \subset A$ & - & - \\
\hline
\end{tabular}

Unterschiedliche Klein- und Großbuchstaben indizieren signifikante Unterschiede zwischen den Saatstärken bzw. Behandlungsvarianten, 1999/00 und 2000/01: ANOVA, Tukey-Test, 2001/02: Mann-Whitney U-Test, $p<0,05$

Tab. 45: Mittleres Tausendkorngewicht $[\mathrm{g}]( \pm \mathrm{SE})$ in Abhängigkeit von der Saatstärke in den Anbaujahren 1999/00 und 2000/01 am Standort Weendelsbreite und im Anbaujahr 2001/02 am Standort Otto-Hahn-Straße

\begin{tabular}{llllll}
\hline Saatstärke & \multicolumn{1}{c}{$1999 / 00$} & \multicolumn{2}{c}{$2000 / 01$} & \multicolumn{2}{c}{$2001 / 02$} \\
& behandelt & behandelt & unbehandelt & behandelt & unbehandelt \\
& $\mathrm{X}_{\mathrm{m}} \pm \mathrm{SE}$ & $\mathrm{X}_{\mathrm{m}} \pm \mathrm{SE}$ & $\mathrm{X}_{\mathrm{m}} \pm \mathrm{SE}$ & $\mathrm{X}_{\mathrm{m}} \pm \mathrm{SE}$ & $\mathrm{X}_{\mathrm{m}} \pm \mathrm{SE}$ \\
\hline $30 \mathrm{Körner} / \mathrm{m}^{2}$ & $4,6 \pm 0,1$ a & $4,5 \pm 0,0$ a A & $4,6 \pm 0,0$ a A & $4,4 \pm 0,1$ a A & $4,2 \pm 0,1$ a $\mathrm{A}$ \\
$60 \mathrm{Körner} / \mathrm{m}^{2}$ & $4,6 \pm 0,1$ a & $4,4 \pm 0,0$ a A & $4,5 \pm 0,0$ a A & $4,5 \pm 0,0$ a A & $4,3 \pm 0,1$ a A \\
$90 \mathrm{Körner} / \mathrm{m}^{2}$ & $4,6 \pm 0,0$ a & $4,5 \pm 0,1$ a A & $4,5 \pm 0,1$ a A & - & - \\
\hline
\end{tabular}

Unterschiedliche Klein- und Großbuchstaben indizieren signifikante Unterschiede zwischen den Saatstärken bzw. Behandlungsvarianten, 1999/00 und 2000/01: ANOVA, Tukey-Test, 2001/02: Mann-Whitney U-Test, $p<0,05$

\subsubsection{Beziehung zwischen den Ertragsparametern der Pflanzen und der Pflanzen- dichte}

Zwischen dem Kornertrag/ha bzw. dem TKG und der Pflanzendichte ergaben sich keine signifikanten Korrelationen (Tab. 46). Dies galt sowohl für die Insektizid-behandelten als auch für die unbehandelten Teilflächen. Für den Einzelpflanzenertrag hingegen fanden sich in allen Versuchen und Behandlungsstufen hochsignifikante und eng korrelierte, negative Beziehungen zur Pflanzenzahl $/ \mathrm{m}^{2}$. Eine Reduktion der Pflanzendichte führte zu einer deutlichen Steigerung des Ertrages/Pflanze. 
Tab. 46: Korrelationen zwischen den verschiedenen Ertragsparametern und der Pflanzendichte im Juli 2000 und 2001 am Standort Weendelsbreite und im Juli 2002 am Standort Otto-Hahn-Straße

\begin{tabular}{lccccc}
\hline \multicolumn{2}{c}{$\begin{array}{c}\text { Korrelierende } \\
\text { Merkmale }\end{array}$} & Jahr & $r$ & $\mathrm{p}$ & $\mathrm{B}[\%]$ \\
\hline Kornertrag [dt/ha] & - Pflanzendichte & 2000 & $+0,18$ & 0,489 & 3,1 \\
behandelt & behandelt & 2001 & $+0,33$ & 0,173 & 11,1 \\
& & 2002 & $-0,54$ & 0,134 & 28,8 \\
Kornertrag [dt/ha] & - Pflanzendichte & 2000 & - & & \\
unbehandelt & unbehandelt & 2001 & $+0,10$ & 0,683 & 1,1 \\
& & 2002 & $-0,13$ & 0,738 & 1,7 \\
\hline TKG [g] & Pflanzendichte & 2000 & $+0,06$ & 0,810 & 0,4 \\
behandelt & behandelt & 2001 & $-0,21$ & 0,415 & 4,3 \\
& & 2002 & $+0,41$ & 0,269 & 17,1 \\
TKG [g] & Pflanzendichte & 2000 & - & & \\
unbehandelt & unbehandelt & 2001 & $-0,19$ & 0,453 & 3,7 \\
& & 2002 & $-0,06$ & 0,874 & 0,4 \\
\hline Ertrag/Pflanze [g/Pfl.] & - Pflanzendichte & 2000 & $-0,90$ & 0,000 & 80,2 \\
behandelt & behandelt & 2001 & $-0,95$ & 0,000 & 89,3 \\
& & 2002 & $-0,98$ & 0,000 & 95,1 \\
Ertrag/Pflanze [g/Pfl.] & - Pflanzendichte & 2000 & - & & \\
unbehandelt & unbehandelt & 2001 & $-0,96$ & 0,000 & 91,4 \\
& & 2002 & $-0,92$ & 0,001 & 85,2 \\
\hline
\end{tabular}

\subsubsection{Beziehung zwischen den Ertragsparametern der Pflanzen und der Pflanzen- architektur}

Im Folgenden wird nur die Beziehung zwischen dem Einzelpflanzenertrag und den Wachstumsparametern der Rapspflanzen überprüft, da für die Ertragsparameter Flächenertrag und TKG keine signifikanten Beziehungen zur Pflanzendichte festgestellt wurden (Kap. 3.4.2) und die Pflanzendichte die Entwicklung der Pflanzenarchitektur signifikant beeinflusst (Kap. 3.1.4, Kap. 3.1.5). Die Daten der Wachstumsparameter der Pflanzen an den Probeterminen im November, April und Mai sowie die Daten des Ertrages pro Pflanze stammten mit nur einer Ausnahme aus den Insektizid-unbehandelten Teilflächen. Lediglich im Versuch 1999/00 kamen die Ergebnisse der frühsommerlichen Pflanzenentwicklung (April und Mai) und des Ertrages pro Pflanze aus den behandelten Parzellen.

In Tabelle 47 sind die linearen Beziehungen zwischen dem Ertrag/Pflanze und verschiedenen Wachstumsparametern der Pflanzen in den Monaten November (Tab. 47a), April (Tab. 47b) und Mai (Tab. 47c) in den drei Versuchsjahren 1999/00 bis 2001/02 wiedergegeben. Zwischen dem Ertrag/Pflanze und den Pflanzenparametern ergaben sich mit nur wenigen Ausnahmen signifikante, eng korrelierte positive Beziehungen. Lediglich für die Sprosslänge bestand im 
November der Versuche 2000/01 und 2001/02 kein statistischer Zusammenhang. Weiterhin bestand im April 2000 und 2002 sowie im Mai 2002 keine signifikante Beziehung zur Sprosslänge. Auch für den Sprossdurchmesser in $50 \mathrm{~cm}$ Höhe und die Anzahl primärer Seitentriebe im Mai 2002 konnten keine statistisch gesicherten Zusammenhänge hergestellt werden.

Tab. 47a: Korrelationen zwischen dem Ertrag/Pflanze und verschiedenen Wachstumsparametern der Pflanzen im November in den Versuchen 1999/00 und 2000/01 am Standort Weendelsbreite und im Versuch 2001/02 am Standort Otto-Hahn-Straße

\begin{tabular}{|c|c|c|c|c|}
\hline $\begin{array}{l}\text { Korrelierende } \\
\text { Merkmale* }^{*}\end{array}$ & Versuch & $r$ & $\mathrm{p}$ & $\mathrm{B}[\%]$ \\
\hline 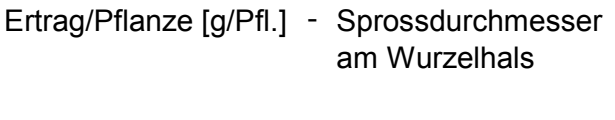 & $\begin{array}{l}1999 / 00 \\
2000 / 01 \\
2001 / 02\end{array}$ & $\begin{array}{l}+0,81 \\
+0,92 \\
+0,75\end{array}$ & $\begin{array}{l}0,000 \\
0,000 \\
0,019\end{array}$ & $\begin{array}{l}65,1 \\
85,2 \\
56,8\end{array}$ \\
\hline Ertrag/Pflanze [g/Pfl.] - Anzahl Blätter & $\begin{array}{l}1999 / 00 \\
2000 / 01 \\
2001 / 02\end{array}$ & $\begin{array}{l}+0,80 \\
+0,95 \\
+0,77\end{array}$ & $\begin{array}{l}0,000 \\
0,000 \\
0,014\end{array}$ & $\begin{array}{l}63,7 \\
90,2 \\
60,1\end{array}$ \\
\hline Ertrag/Pflanze [g/Pfl.] - Sprosslänge & $\begin{array}{l}1999 / 00 \\
2000 / 01 \\
2001 / 02\end{array}$ & $\begin{array}{l}+0,91 \\
+0,39 \\
-0,11\end{array}$ & $\begin{array}{l}0,000 \\
0,111 \\
0,766\end{array}$ & $\begin{array}{c}82,7 \\
14,9 \\
1,3\end{array}$ \\
\hline
\end{tabular}

* 1999/00 wurden die vorwinterlichen pflanzlichen Merkmale in den Insektizid-unbehandelten, der Ertrag/Pflanze in den behandelten Teilflächen untersucht, 2000/01 und 2001/02 wurden alle Merkmale in den unbehandelten Teilflächen untersucht

Tab. 47b: Korrelationen zwischen dem Ertrag/Pflanze und verschiedenen Wachstumsparametern der Pflanzen im April in den Versuchen 2000 und 2001 am Standort Weendelsbreite und im Versuch 2002 am Standort Otto-Hahn-Straße

\begin{tabular}{|c|c|c|c|c|}
\hline $\begin{array}{c}\text { Korrelierende } \\
\text { Merkmale* }^{*}\end{array}$ & Versuch & $r$ & $p$ & $\mathrm{~B}[\%]$ \\
\hline $\begin{array}{c}\text { Ertrag/Pflanze [g/Pfl.] - Sprossdurchmesser } \\
\text { am Wurzelhals }\end{array}$ & $\begin{array}{l}2000 \\
2001 \\
2002\end{array}$ & $\begin{array}{r}+0,69 \\
+0,95 \\
+0,93\end{array}$ & $\begin{array}{l}0,002 \\
0,000 \\
0,000\end{array}$ & $\begin{array}{l}47,0 \\
90,1 \\
87,4\end{array}$ \\
\hline Ertrag/Pflanze [g/Pfl.] - Anzahl Blätter & $\begin{array}{l}2000 \\
2001 \\
2002\end{array}$ & $\begin{array}{l}+0,65 \\
+0,73 \\
+0,72\end{array}$ & $\begin{array}{l}0,004 \\
0,001 \\
0,027\end{array}$ & $\begin{array}{l}41,8 \\
54,0 \\
52,4\end{array}$ \\
\hline Ertrag/Pflanze [g/Pfl.] - Sprosslänge & $\begin{array}{l}2000 \\
2001 \\
2002\end{array}$ & $\begin{array}{l}+0,41 \\
+0,91 \\
+0,62\end{array}$ & $\begin{array}{l}0,086 \\
0,000 \\
0,071\end{array}$ & $\begin{array}{l}17,0 \\
83,1 \\
38,9\end{array}$ \\
\hline Ertrag/Pflanze [g/Pfl.] - Anzahl Seitentriebe & $\begin{array}{l}2000 \\
2001 \\
2002\end{array}$ & $\begin{array}{l}+0,76 \\
+0,91 \\
+0,92\end{array}$ & $\begin{array}{l}0,000 \\
0,000 \\
0,001\end{array}$ & $\begin{array}{l}58,1 \\
81,9 \\
85,6\end{array}$ \\
\hline $\begin{aligned} \text { Ertrag/Pflanze [g/Pfl.] - } & \text { Klassen mit unterschied- } \\
& \text { licher Seitentrieblänge }\end{aligned}$ & $\begin{array}{l}2000 \\
2001 \\
2002\end{array}$ & $\begin{array}{c}+0,79 \\
+0,86 \\
-\end{array}$ & $\begin{array}{l}0,000 \\
0,000\end{array}$ & $\begin{array}{l}62,6 \\
73,2\end{array}$ \\
\hline $\begin{aligned} \text { Ertrag/Pflanze [g/Pfl.] - } & \text { Länge der } \\
& \text { Seitentriebe }\end{aligned}$ & $\begin{array}{l}2000 \\
2001 \\
2002\end{array}$ & $\begin{array}{c}- \\
+0,91 \\
+0,81\end{array}$ & $\begin{array}{l}0,000 \\
0,008\end{array}$ & $\begin{array}{l}82,0 \\
66,0\end{array}$ \\
\hline $\begin{array}{c}\text { Ertrag/Pflanze [g/Pfl.] - Durchmesser der } \\
\text { Seitentriebe }\end{array}$ & $\begin{array}{l}2000 \\
2001 \\
2002\end{array}$ & $\begin{array}{c}- \\
+0,91 \\
+0,86\end{array}$ & $\begin{array}{l}0,000 \\
0,003\end{array}$ & $\begin{array}{l}82,1 \\
73,9\end{array}$ \\
\hline
\end{tabular}

* 2000 wurden alle Merkmale in den Insektizid-behandelten Teifflächen, 2001 und 2002 in den unbehandelten Teilflächen untersucht 
Tab. 47c: Korrelationen zwischen dem Ertrag/Pflanze und verschiedenen Wachstumsparametern der Pflanzen im Mai in den Versuchen 2000 und 2001 am Standort Weendelsbreite und im Versuch 2002 am Standort Otto-Hahn-Straße

\begin{tabular}{|c|c|c|c|c|c|}
\hline $\begin{array}{r}\text { Korrelie } \\
\text { Merkn }\end{array}$ & $\begin{array}{l}\text { erende } \\
\text { nale* }\end{array}$ & Versuch & $r$ & $\mathrm{p}$ & $\mathrm{B}[\%]$ \\
\hline Ertrag/Pflanze [g/Pfl.] & $\begin{array}{l}\text { - Sprossdurchmesser } \\
\text { am Wurzelhals }\end{array}$ & $\begin{array}{l}2000 \\
2001 \\
2002\end{array}$ & $\begin{array}{l}+0,86 \\
+0,94 \\
+0,69\end{array}$ & $\begin{array}{l}0,000 \\
0,000 \\
0,037\end{array}$ & $\begin{array}{l}74,5 \\
88,9 \\
48,3\end{array}$ \\
\hline Ertrag/Pflanze [g/Pfl.] & $\begin{array}{l}\text { - Sprossdurchmesser } \\
\text { in } 50 \mathrm{~cm} \text { Höhe }\end{array}$ & $\begin{array}{l}2000 \\
2001 \\
2002\end{array}$ & $\begin{array}{l}+0,82 \\
+0,96 \\
+0,48\end{array}$ & $\begin{array}{l}0,000 \\
0,000 \\
0,193\end{array}$ & $\begin{array}{l}66,5 \\
91,4 \\
22,7\end{array}$ \\
\hline Ertrag/Pflanze [g/Pfl.] & $\begin{array}{l}\text { - Anzahl primärer } \\
\text { Seitentriebe }\end{array}$ & $\begin{array}{l}2000 \\
2001 \\
2002\end{array}$ & $\begin{array}{l}+0,85 \\
+0,92 \\
+0,64\end{array}$ & $\begin{array}{l}0,000 \\
0,000 \\
0,063\end{array}$ & $\begin{array}{l}72,7 \\
84,6 \\
40,6\end{array}$ \\
\hline Ertrag/Pflanze [g/Pfl.] & $\begin{array}{l}\text { - Anzahl sekundärer } \\
\text { Seitentriebe }\end{array}$ & $\begin{array}{l}2000 \\
2001 \\
2002\end{array}$ & $\begin{array}{l}+0,90 \\
+0,87 \\
+0,78\end{array}$ & $\begin{array}{l}0,000 \\
0,000 \\
0,013\end{array}$ & $\begin{array}{l}81,4 \\
76,3 \\
61,1\end{array}$ \\
\hline Ertrag/Pflanze [g/Pfl.] & $\begin{array}{l}\text { - Anzahl } \\
\text { Seitentriebe }\end{array}$ & $\begin{array}{l}2000 \\
2001 \\
2002\end{array}$ & $\begin{array}{c}- \\
- \\
+0,75\end{array}$ & 0,020 & 55,9 \\
\hline Ertrag/Pflanze [g/Pfl.] & - Sprosslänge & $\begin{array}{l}2000 \\
2001 \\
2002\end{array}$ & $\begin{array}{l}+0,74 \\
+0,82 \\
+0,43\end{array}$ & $\begin{array}{l}0,001 \\
0,000 \\
0,246\end{array}$ & $\begin{array}{l}54,9 \\
67,5 \\
18,6\end{array}$ \\
\hline
\end{tabular}

* 2000 wurden alle Merkmale in den Insektizid-behandelten Teilflächen, 2001 und 2002 in den unbehandelten Teilflächen untersucht 


\section{Diskussion}

Die vorliegende Arbeit beschäftigt sich mit den Auswirkungen unterschiedlicher Aussaatstärken von Winterraps und der daraus resultierenden Pflanzenarchitektur auf Befallsdichte, Verteilungsmuster und Schadwirkung der stängelminierenden Schadinsekten sowie den Ertrag. Dabei war auch die Bewertung reduzierter Pflanzendichten hinsichtlich der Kompensationsfähigkeit der Rapspflanzen gegenüber dem Schädlingsbefall und die sich daraus ergebenden Konsequenzen für die Ertragssicherheit von Interesse. Zu diesem Zweck wurden die Befallsdichten und die räumlich-zeitliche Verteilung der Triebschädlinge mit Gelb- und Fangschalen, Bodenphotoeklektoren, Boden- und Pflanzenproben erfasst. Die Ertragsparameter wurden durch Beerntung von Exaktparzellen ermittelt. Anhand von Korrelations- und Regressionsrechnungen erfolgte eine Analyse des Zusammenhanges zwischen der Befallsdichte der Triebschädlinge sowie den Pflanzen- und Ertragsparametern. Begleitend wurden die Pflanzendichten und die Wachstumsparameter der Rapspflanzen erfasst.

\subsection{Entwicklung der Pflanzendichten}

Die unterschiedlichen Pflanzendichten von Winterraps in den Versuchsvarianten wurden in den drei Versuchsjahren durch entsprechend abgestufte Aussaatstärken eingestellt. Die im ersten Versuchsjahr 1999/00 in allen Saatvarianten um 8 bis 13 Pflanzen/m² gegenüber der Aussaatdichte erhöhten Pflanzendichten waren die Folge der überliegenden Rapssaat früherer Rapsanbaujahre. Da die Erhöhung der Pflanzendichte in allen Saatstärkevarianten nahezu gleichmäßig erfolgte, konnten die abgestuften Dichten für die Untersuchung dennoch in vollem Umfang genutzt werden. Die Optimierung von Saatbettbereitung und Aussaattechnik ermöglichte es in den folgenden Versuchsjahren 2000/01 und 2001/02, die angestrebten unterschiedlichen Pflanzendichten zum Versuchsbeginn im Herbst relativ exakt einzustellen. In zahlreichen Feldversuchen, in denen der Einfluss unterschiedlicher Rapsdichten auf Ertrag und Schaderregerbefall in der Vergangenheit untersucht wurde, wurden die erwünschten Pflanzendichten erst nach dem Auflaufen des Rapses durch aufwändige Handvereinzelung eingestellt (HenNing 1979, Boelcke 1981, Stoy 1983, Broschewitz 1985, GeißLer \& StOy 1987, Sierts 1987, McGRegor 1987, WAHMHOFF 2000). Dabei kann es zu Beschädigungen der Pflanzen kommen. Neuere Untersuchungen haben gezeigt, dass die Pflanzenzahl pro $\mathrm{m}^{2}$ mit der modernen Drillsaattechnik sehr gut über die Saatdichte kontrolliert werden kann (DIEPENBROCK 1999). Die Einstellung unterschiedlicher Pflanzendichten über die Wahl der Saat- 
stärke war in dieser Untersuchung bei Kenntnis der standortbedingten Einflüsse gut möglich. Neuere Feldversuche von Aigner (2003) haben gezeigt, dass bei einer optimalen Saatbettbereitung und der Verwendung der Einzelkornsätechnik ein noch höherer und gleichmäßigerer Feldaufgang erreicht werden kann als mit der hier verwendeten Drillsaattechnik.

In Abhängigkeit von der Saatstärke kann es im gesamten Entwicklungsverlauf des Rapsbestandes zu unterschiedlichen Pflanzenverlusten kommen. Bei höheren Ausgangspflanzendichten kommt es häufig während des Winters zu höheren Pflanzenverluste als bei niedrigeren Pflanzendichten (Morice 1960, STOY 1981, 1983, GeißLER \& STOY 1987, SiERTS et al. 1987, Schulz 1994, Sauermann 1997, Aigner 2003). Eine erhöhte Pflanzenmortalität wurde in den beiden ersten Versuchsjahren dieser Arbeit nur bei der hohen Saatstärke $\left(90 \mathrm{Kö.} / \mathrm{m}^{2}\right)$ registriert. Als mögliche Ursachen für den Pflanzenverlust kommen ein unterschiedlicher Befall der Rapspflanzen durch die Larven des Rapserdflohs oder eine unterschiedliche Kältetoleranz bei verschiedenen Pflanzendichten in Frage.

Ein Einfluss des Befalls durch die Larven des Rapserdflohs auf die Pflanzenmortalität wurde von Schulz (1993), NiLSSON (2002), PAUL (2003) (vgl. Kap. 4.6) festgestellt. In vorliegender Arbeit war eine deutliche Absenkung der Pflanzenverluste durch die chemische Bekämpfung des Rapserdflohs lediglich im Versuch 2000/01 bei der Saatstärke 90 Kö./m² zu verzeichnen. Die Pflanzenverluste lagen mit 19,4 \% in den unbehandelten Parzellen deutlich höher als in den behandelten mit 11,4\%. Dies zeigt, dass der Rapserdflohbefall in diesem Versuchsjahr zur Reduktion der Pflanzendichten beigetragen hat. Für den ersten Versuch 1999/00 ist keine Aussage zur Rolle des Rapserdflohes möglich, da die gesamten Parzellenflächen mit Insektizid behandelt wurden. Die Pflanzenverluste beliefen sich trotz der Insektizidbehandlung auf $20 \%$. Dies macht deutlich, dass neben dem Schädlingsbefall auch andere Faktoren zur Auswinterung in dichten Rapsbeständen beitragen.

Daneben ist die starke Auswinterung der Pflanzen bei der hohen Saatstärke $90 \mathrm{Kö.} / \mathrm{m}^{2}$ in den beiden ersten Versuchsjahren vermutlich zu einem erheblichen Teil auf die wesentlich schwächere Entwicklung der dichtstehenden Einzelpflanzen in dieser Variante vor Winter zurückzuführen. Das Wurzel- und Blattwachstum sowie die Trockenmassebildung der Pflanzen im Herbst werden bei höheren Saatstärken durch die engeren Standräume der Pflanzen eingeschränkt (HenNing 1979, Stoy 1981, DenNert \& FischBeck 2000). Der für die Pflanzen zur Verfügung stehende Standraum beeinflusst ihre Kältetoleranz insofern, als bei den dichtstehenden Pflanzen aufgrund der intraspezifischen Konkurrenz zwischen den Pflanzen um den Wachstumsfaktor Licht die Streckung der Sprossachse früher einsetzt, wobei sich der Vegetationspunkt vom schützenden Erdboden entfernt und damit frostanfälliger wird (HENNING 1983, 
DenNerT \& FischBecK 2000, Aigner 2003). Geisler (1981), STOY (1981) und SiERTS (1987) stellten bei Untersuchungen mit verschiedenen Reihenabständen fest, dass die Rapspflanzen sich bei geringen Reihenweiten infolge der größeren Abstände zur Nachbarpflanze in den Reihen kräftiger entwickelten und weniger von Auswinterungsverlusten betroffen waren als die Pflanzen großer Reihenweiten. Eine stärkere Reduktion der Pflanzenzahl ist bei hohen Pflanzendichten vor allem dann zu erwarten, wenn es nach sehr frühem Aussaattermin bereits vor dem Winter zu einem starken Längenwachstum der Sprossachse kommt (BoELCKE 1981). In der vorliegenden Untersuchung war eine deutliche Abnahme der Pflanzenzahl $/ \mathrm{m}^{2}$ infolge einer bereits vor dem Winter einsetzenden Stängelbildung im Versuch 2000/01 lediglich bei den Pflanzen der hohen Saatstärke $90 \mathrm{Kö.} / \mathrm{m}^{2}$ festzustellen, so dass dieser Faktor neben dem Rapserdflohbefall für die deutliche Reduktion der Pflanzendichte in diesem Versuchsjahr verantwortlich ist. In den beiden anderen Versuchsjahren wurde die kritische Länge der Sprossachse von etwa 20 mm (MAKOWSKI 1995) in keiner der Saatstärkenvarianten überschritten.

Im Versuchsjahr 2001/02 war allerdings auch bei den geringen Saatstärken (30 und $60 \mathrm{Kö.} / \mathrm{m}^{2}$ ) eine statistisch gesicherte Reduktion der Pflanzendichte über Winter zu beobachten. Diese Pflanzenverluste wurden jedoch vor allem durch Kaninchen und Feldmäuse verursacht, die sowohl in der Umgebung als auch auf der Versuchsfläche selbst zahlreich zu beobachten waren. Eine Auswinterung der Pflanzen durch die Schadwirkung der Rapserdflohlarven kann in diesem Versuch ausgeschlossen werden, da sowohl in den Insektizid-behandelten als auch in den unbehandelten Teilflächen des Versuches eine ähnliche Pflanzenreduktion bis zum Frühjahr zu verzeichnen war.

\subsection{Einfluss der Pflanzendichte auf die Wachstumsparameter der Pflanzen}

Durch die Variation der Saatstärke kann die Einzelpflanzenentwicklung gezielt beeinflusst werden. Wie bereits von anderen Autoren festgestellt wurde (KöRTING 1942, SCHRIMPF 1954, HenNing 1979, GeißLer \& Henning 1981a, Stoy 1981, SCARISBrick et al. 1982, Geisler \& Stoy 1987, Schulz 1994, Makowski 1995, МомоH \& ZHOU 2001, Aigner 2003), bewirkt die Reduktion der Saatstärke bzw. der Pflanzendichte eine deutliche Zunahme der Anzahl der Blätter und Seitentriebe, der Pflanzenlänge, des Wurzelhalsdurchmessers und der Trockenmasseproduktion der Einzelpflanzen. Die Unterschiede in der Ausbildung dieser Wachstumsparameter zwischen den drei Versuchen verweisen auf jahresbedingte Einflüsse, die vor allem auf unterschiedliche Witterungs- und Wachstumsbedingungen im Herbst zurückzuführen sind. Daneben waren auch die Sorteneigenschaften der im 1. Jahr angebauten Linienrapssorte Mo- 
hican und der im 2. und 3. Versuchsjahr verwendeten Hybridrapssorte Artus für das unterschiedliche Wachstum der Pflanzen verantwortlich. Die vorwinterliche Entwicklung der Sprosslänge wurde insbesondere bei der Liniensorte signifikant durch die Pflanzendichte beeinflusst. Die Pflanzen der wuchsstarken Hybridrapssorte hingegen wiesen unabhängig von der Pflanzendichte eine nahezu gleiche Länge der Hauptsprossachse auf. Diese Sorteneigenschaft des Hybridrapses, die auf der starken Wüchsigkeit dieser Sorten beruht (SAUERMANN \& Gronow 1999, Aigner 2003), war lediglich im Herbst festzustellen. Im Frühjahr stieg die Pflanzenlänge mit abnehmender Bestandesdichte bei der Linien- und Hybridrapssorte signifikant an.

Ein Einfluss der Witterung auf die Entwicklung der Pflanzen war bei der Hybridrapssorte im 3. Versuchsjahr zu beobachten. Die nasskalte Witterung während der Keimungsphase hatte einen verzögerten Auflauf der Saat zur Folge. Durch die warme Witterung im Oktober in Kombination mit der hohen Vitalität und Wüchsigkeit der Hybridsorte wurde zwar noch eine ausreichende Entwicklung der Pflanzen vor dem Winter erreicht, sie entsprach allerdings lediglich der Entwicklung der Linienrapssorte im 1. Versuchsjahr. Erst im April (BBCH 55-59) zeigte die später aufgelaufene Hybridrapssorte eine schnellere Entwicklung als die Linienrapssorte. Im Mai glich sie sich der Morphologie der früh aufgelaufenen Hybridrapssorte (BBCH 67-69) des 2. Jahres an. Das raschere Wachstum der Hybridrapssorte im 3. Versuchsjahr ist vermutlich darauf zurückzuführen, dass den Pflanzen aufgrund der Auswinterungsverluste mehr Standraum zur Verfügung stand. AULD et al. (1984) machten bei Versuchen mit unterschiedlichen Saatzeitpunkten von Winterraps eine ähnliche Beobachtung: Nur die Spätsaatvarianten, die im Winter besonders hohe Pflanzenverluste aufwiesen, konnten die noch zu Blühbeginn bestehende geringere Verzweigung im weiteren Verlauf der Vegetationsperiode bis zu der Verzweigungsdichte der frühen Saattermine angleichen. Außerdem ist zu berücksichtigen, dass diese Ergebnisse mit einer Hybridrapssorte ermittelt wurden, die aufgrund ihrer Wüchsigkeit wesentlich deutlicher auf die sich ändernden Konkurrenzverhältnisse im Bestand reagieren kann als die weniger wuchsstarke Linienrapssorte.

Die vorliegende Untersuchung verdeutlicht, dass auch die Anzahl schotentragender Seitentriebe eine starke Abhängigkeit von der Pflanzendichte aufweist. Die Fähigkeit kräftiger Pflanzen, die vorhandenen Blüten an einer größeren Anzahl von Seitentrieben bis zur Schote zu entwickeln, beruhte auf der unterschiedlichen Standraumverteilung im Frühjahr. So beobachteten auch SCHRIMPF (1954) und STOY (1983) einen höheren Schotenansatz bei kräftigen Pflanzen mit hohen Pflanzengewichten und weiterem Standraum. Nach STOY (1983) könnte der höhere Schotenansatz der Pflanzen auf die höhere Lichtintensität im weniger dichten Bestand und 
damit auf die Ausbildung hoher Trockenmassen und auf eine größere Blattfläche zurückzuführen sein.

Die Beobachtungen im Frühjahr zeigten darüber hinaus, dass die wesentlich bessere vorwinterliche Einzelpflanzenentwicklung der Hybridrapssorte im 2. Jahr ein relativ früh einsetzendes, intensiveres Wachstum der Haupt- und Seitensprossachsen im Frühjahr zur Folge hatte. Ein ähnliches Ergebnis war im 1. und 3. Versuchsjahr nur bei den Pflanzen der geringen Saatstärke festzustellen: Sie entwickelten sich gegenüber den Pflanzen der hohen Saatstärken vor dem Winter weiter, so dass im Frühjahr ein schnelleres Längenwachstum der Haupttriebe und eine relativ frühe Ausbildung der Seitentriebe möglich waren. Auch STOY (1983) machte ähnliche Beobachtungen zum Sprosswachstum der Pflanzen im Frühjahr und stellte mit zunehmender Pflanzendichte einen verzögerten Anstieg der Trockenmasseproduktion fest. VULLIOUD (1974) und MENDHAM \& SCOTT (1975) fanden bei spät gesäten Rapspflanzen eine nur langsame Zunahme der Wurzeltrockenmasse im Frühjahr. Die Entwicklung dieser Pflanzen ist mit der Entwicklung der Pflanzen aus den hohen Aussaatdichten in eigener Untersuchung durchaus vergleichbar.

Die zum großen Teil negativen Korrelationen zwischen der Pflanzendichte und den Wachstumsparametern der Rapspflanzen vor und nach der Überwinterung bestätigen die oben angeführten Überlegungen: Mit abnehmender Saatstärke bzw. Pflanzendichte tritt eine Förderung der Einzelpflanzenentwicklung ein. Die fehlenden Korrelationen zwischen der Pflanzendichte und den Pflanzenparametern Sprosslänge und Sprossdurchmesser in $50 \mathrm{~cm}$ Höhe sind vermutlich auf die oben genannten Sortenunterschiede und auf die Unterschiede im Befallsdruck mit den Triebschädlingen in den entsprechenden Versuchsjahren zurückzuführen. Die Entwicklung dieser beiden Parameter im Jahr 2002 korrespondierte mit einem relativ hohen Befall von C. napi (Kap. 3.3.3), der sich stark auf Sprosslänge und -durchmesser auswirkt (ALFORD et al. 2003, PAUl 2003). Aus den engen Beziehungen zwischen der Pflanzendichte und den Pflanzenparametern des Hybridrapses gegenüber dem Linienraps kann geschlossen werden, dass der Hybridraps infolge seiner Wüchsigkeit und Vitalität besser auf unterschiedliche Konkurrenzbedingungen im Bestand reagieren kann als der Linienraps.

\subsection{Beurteilung der Methoden zur Erfassung der Triebschädlinge}

Die Wahl der Erfassungsmethode beeinflusst maßgeblich die ermittelten Ergebnisse, da jede freilandökologische Methode spezifische Grenzen aufweist und einzelne Tiergruppen oder arten von der jeweiligen Methode mit unterschiedlicher Effizienz erfasst werden (SUNDER- 
LAND 1987, TROMMER 1991). In der vorliegenden Arbeit kamen für die Bestimmung der Befallsentwicklung von $P$. chrysocephala, $C$. napi und $C$. pallidactylus sowohl Methoden zur Erfassung der Phänologie und Aktivitätsdichte (Gelbschalen, Fangschalen) als auch Methoden zur näherungsweisen Dichteerfassung (Photoeklektoren, Extraktion aus Bodenproben, Präparation von Pflanzenproben) zum Einsatz. Mit Ausnahme der Gelbschalen, die außerhalb der Versuchsparzellen installiert wurden und lediglich der Kontrolle des Aktivitätsbeginns und verlaufs der genannten Triebschädlinge dienten, fanden die übrigen Methoden innerhalb der Parzellen mit unterschiedlichen Pflanzendichten Verwendung.

\section{$\underline{\text { Gelbschalen }}$}

Der Gelbschalenfang erwies sich in den drei Untersuchungsjahren als eine geeignete Methode zur Erfassung des Erstauftretens und der Zuwanderungsaktivität der drei endophagen Rapsschädlinge. Obwohl P. chrysocephala nicht durch die gelbe Farbe der Fangschalen angelockt wird (SCHRÖDTER \& NOLTE 1954), sondern durch seine springende Fortbewegungsweise zufällig in die Gelbschalen gelangt, bieten sich die Gelbschalenfänge nach HOßFELD (1993) dennoch als Hilfsmittel bei der Bekämpfungsentscheidung für diesen Schädling an. Seinen Untersuchungen zufolge lässt sich zwischen den Schalenfängen und dem späteren Larvenbesatz in den Rapspflanzen durchaus ein qualitativer Zusammenhang herstellen. Hohe Käferzahlen sind zwar nicht immer mit einer hohen Larvendichte verbunden, geringe Fangzahlen zeigen hingegen zuverlässig geringe Larvenzahlen an. Wie die Ergebnisse von insgesamt 49 Standorten zeigten, zog ein Käferfang unterhalb des Schwellenwertes von 50 Käfern je Gelbschale innerhalb von 3 Wochen während der Hauptzuwanderung (BBCH 14-16) in keinem Fall einen kritischen Larvenbesatz (Schadensschwellenwert: 3 - 5 Larven/Pflanze) nach sich (HOßFELD 1993).

Bei C. napi und C. pallidactylus, die erst im Frühjahr in die Rapsbestände einfliegen, erlauben die Fangzahlen der Gelbschalen hingegen nur eingeschränkt Rückschlüsse auf die Befallsstärke der Pflanze (Lauenstein 1993, Seigner 1993, Debouzie \& Ballanger 1993). In den Untersuchungen von ULBER (1994) in den Jahren 1989 bis 1993 wurde zwar ein positiver Zusammenhang zwischen den während der gesamten Zuflugperiode aufsummierten Gelbschalenfangzahlen von C. napi und C. pallidactylus und den nach Abschluss des Zufluges ermittelten Abundanzen der Larven pro $\mathrm{m}^{2}$ festgestellt. In den eigenen Untersuchungen konnte jedoch keine positive Beziehung zwischen den Gelbschalenfangzahlen und der Larvenzahl pro $\mathrm{m}^{2}$ aufgezeigt werden (Tab. A-6, Anhang). Die Larvenabundanz wurde offenbar durch die Mortalität der Käfer nach der Zuwanderung und durch den Einfluss der Witterung auf die Eiablage 
und Eimortalität von Jahr zu Jahr unterschiedlich beeinflusst. Für beide Schädlingsarten liegen lediglich „Hilfswerte“ für die Bekämpfungsentscheidung vor (10 Käfer pro Gelbschale in 3 Tagen), die einer weiteren Überprüfung bedürfen (GARBE et al. 1996). Die Gelbschalenfänge liefern dennoch wichtige Hinweise auf den Beginn der Besiedlung und damit zur optimalen Terminierung der Insektizidbehandlungen im Frühjahr. Nach BÜCHS (1997) ist erst nach dem Auftreten eiertragender Weibchen in den Gelbschalen eine Insektizidanwendung sinnvoll (Bekämpfungsschwelle: $>20 \%$ der Käfer einer Stichprobe eiertragend). In vielen Fällen ist es möglich, mit einer einzigen Behandlung sämtliche auftretenden Schädlinge im Raps ausreichend zu reduzieren (BÜCHS 1997).

\section{$\underline{\text { Fangschalen }}$}

Der Einsatz von Fangschalen am Boden der Parzellen ermöglichte eine genauere Darstellung der Aktivitätsdichte von $P$. chrysocephala in Abhängigkeit von der Pflanzendichte. Da die Fangeffizienz der Schalen sehr stark von der Aktivität der Tiere abhängt (MüHLENBERG 1993), wurde eine mehrtägige Fangperiode gewählt. Zudem berücksichtigt eine mehrtätige Standzeit der Schalen das tageszeitliche Verhalten der Rapserdflöhe. Mit Beginn der Fraßtätigkeit der Käfer bildet sich eine Lichtempfindlichkeit aus, was zu einer Aktivitätsverschiebung auf die Dämmerungs- und Nachtstunden führt (SCHRÖDTER \& NOLTE 1954). Um die Störung der Fangergebnisse durch die sich ab Anfang Oktober langsam schließende Pflanzendecke, insbesondere in den Parzellen mit hohen Pflanzendichten, möglichst gering zu halten, mussten die Schalen regelmäßig kontrolliert und ggf. umgesetzt werden. Der Fang könnte dennoch durch die unterschiedlichen Pflanzendichten in der Umgebung der Fangschale beeinflusst worden sein.

\section{$\underline{\text { Photoeklektoren }}$}

Dieser Fallentyp wird üblicherweise zur Erfassung der Schlupfabundanz positiv phototaktisch reagierender Tiere auf einer durch den Eklektorring begrenzten Fläche eingesetzt. Die dämmerungsaktiven Imagines von $P$. chrysocephala befanden sich zum Zeitpunkt der Probenahme verborgen im oder auf dem Boden und gerieten später im Zuge ihrer Aktivität in die Kopfdosen. Die relative lange Standdauer der Eklektoren hatte das Ziel, alle auf der Fläche eingeschlossenen Tiere zu erfassen. Die höheren Temperaturen im Eklektorzelt haben dies noch begünstigt. Das Aufsetzen der Eklektoren im Bestand ist vermutlich ein Schwachpunkt der Methode, da einige Tiere in diesem Moment aus der vorgesehenen Fläche entkommen konnten. Die Photoeklektoren stellen eine Momentaufnahme der Individuendichte auf der vom 
Eklektorring eingeschlossenen Fläche dar. Dies erklärt möglicherweise die zwischen den Photoeklektoren und Fangschalen mitunter deutlich abweichenden Ergebnisse.

\section{Extraktion der Eier von P. chrysocephala aus Bodenproben}

Eine Möglichkeit zur Abundanzschätzung von edaphischen Lebensformen der Arthropoden bildete die Aufschwemmmethode mit gesättigter Kochsalzlösung aus Bodenproben. Diese Methode wurde bereits zur Erfassung der Eidichte der Brachfliege erfolgreich eingesetzt (SOL 1971). Wie eigene Vorversuche gezeigt haben, können auch die Eier von P. chrysocephala mit einer gesättigten Kochsalzlösung aus dem Bodensubstrat effizient isoliert werden. Die Funktion der Kochsalzlösung besteht darin, die im Boden befindlichen, relativ leichten Eier zum Aufschwimmen zu bringen. Der Nachweis der Erdfloheier war allerdings nur unter einem lichtstarken Binokular möglich. Dabei konnten auch die leeren Eischalen und die Eilarven des Rapserdflohs detektiert werden.

Die Untersuchung der Bodenproben in den Versuchen 2000 und 2001 lieferte teilweise nur relativ ungenaue Ergebnisse. Wie die Tabellen A-7 bzw. A-8 im Anhang zeigen, liegt der Variationskoeffizient für die Eidichten des Rapserdflohs bei verschiedenen Entfernungsstufen von der Pflanze und verschiedenen Saatstärken zwischen 11,5 \% und 28,4 \% im Versuch 2000 und zwischen 6,7 \% und 60,7 \% im Versuch 2001. Der Variationskoeffizient für die Gesamtabundanz der Eier im Versuch 2001 belief sich bei der Saatstärke $30 \mathrm{Kö.} / \mathrm{m}^{2}$ auf 49,8 \% und bei der Saatstärke 60 Kö. $/ \mathrm{m}^{2}$ auf 55,7 \%. Die starke Streuung der Parzellenmittelwerte ist vermutlich auf die aggregierte Verteilung der Eier im Boden zurückzuführen. Erst eine Vervielfachung der Zahl der Proben könnte die Varianz reduzieren. Da das Aufschwemmen der Eier aus den Bodenproben sich als außerordentlich arbeits- und zeitaufwändig herausstellte, war jedoch eine weitere Erhöhung der Stichprobenzahl nicht möglich.

\section{$\underline{\text { Pflanzenproben }}$}

Durch die Präparation der Blätter sowie der Haupt- und Seitentriebe konnten sowohl die Dichte und innerpflanzliche Verteilung der Eier als auch der verschiedenen Larvenstadien der Triebschädlinge in den verschiedenen Saatvarianten ermittelt werden. Zahlreiche Autoren haben diese Methode in früheren Untersuchungen zur Ermittlung der Befallsdichte der endophagen Schadinsekten erfolgreich verwendet (MEUCHE 1940, 1942, KÖRTING 1942, GÜNTHART 1949, Godan 1950, Dosse 1951, Mohr 1960, Palosz 1980, Schulz 1983, Broschewitz 1985, Broschewitz \& DAeBeler 1987, Ulber 1994, BÜCHI 1996, WiLliams et al. 2003). Die Präparation umfangreicher Pflanzenproben ist sehr zeitaufwändig (2 - 3 Stunden pro 10 
Pflanzen); bisher wurde jedoch kein geeignetes Verfahren entwickelt, welches bei geringerem Aufwand Ergebnisse von vergleichbarer Qualität liefern könnte.

\subsection{Phänologie der Triebschädlinge $P$. chrysocephala, $C$. napi und $C$. pallidactylus}

Die Zuwanderung von P. chrysocephala in die Rapsflächen war in allen Versuchsjahren nach Überschreiten einer Temperatur von $16^{\circ} \mathrm{C}$ bis $20^{\circ} \mathrm{C}$ zu verzeichnen; diese Temperaturschwelle wurde auch in Untersuchungen anderer Autoren festgestellt (GODAN 1948, EBBE-NYMANN 1952, SCHRÖDTER \& NOLTE 1954, MOHR 1960, SCHOTT 1962). Diese Temperaturbedingungen waren in den Jahren 1999 und 2000 jeweils in der ersten Septemberdekade erreicht. Die lang anhaltende, kühle Witterung im September 2001 führte zu einer drei Wochen späteren Zuwanderung der Erdflöhe. Auch NolTE (1953) fand bei kühler Witterung im September einen deutlich verzögerten Beginn der Zuwanderung.

Die Zuwanderung des Rapserdflohs war in allen drei Versuchsjahren außerordentlich stark. Die zwischen dem 4- und 6-Blattstadium des Rapses innerhalb von drei Wochen aufsummierten Fangzahlen pro Gelbschale waren mit 180 bzw. 216 Käfern pro Gelbschale in den ersten Jahren um den Faktor 3,6 bzw. 4,3 höher als der Schwellenwert für eine Bekämpfung, der nach HoSSFELD (1993), LAUENSTEIN (1993) und GARBE et al. (1996) bei 50 Käfern pro Gelbschale liegt. Im letzten Jahr blieb die Fangsumme von 40,7 Individuen pro Gelbschale innerhalb von 3 Wochen hingegen unterhalb der Bekämpfungsschwelle.

Wie auch bei $P$. chrysocephala erfolgten der Aktivitätsbeginn und der Zuflug von C. napi und C. pallidactylus in allen drei Versuchsjahren in Abhängigkeit von der Temperatur. Nach BROSCHEWITZ (2001) wird C. napi bereits bei Bodentemperaturen ab $5{ }^{\circ} \mathrm{C}$ auf den vorjährigen Rapsflächen aktiv, während C. pallidactylus erst ab $8-10{ }^{\circ} \mathrm{C}$ in den Winterlagern (Streuschicht von Laubwäldern) erwacht. In allen Versuchsjahren begann die Aktivität von C. napi auf den Überwinterungsfeldern nach Überschreiten einer Lufttemperaturschwelle von $9{ }^{\circ} \mathrm{C}$. Diese Temperatur war in den beiden ersten Jahren in der ersten bzw. letzten Märzdekade, im dritten Versuchsjahr schon in der ersten Februardekade zu verzeichnen. Die Besiedlung der Versuchsflächen durch beide Ceutorhynchus-Arten verlief in allen Jahren nahezu synchron. Der maximale Fang in den Gelbschalen war bei $C$. napi nach dem Überschreiten einer Temperaturschwelle von $12{ }^{\circ} \mathrm{C}$ und einer mittleren Tagestemperatur von $8-9^{\circ} \mathrm{C}$ festzustellen. Nach JOHNEN \& MEIER (2000) lässt sich die Wahrscheinlichkeit des Zufluges von C. pallidactylus in Abhängigkeit von der maximalen Tagestemperatur, der Sonnenscheindauer und der Tagesdurchschnittstemperatur bestimmen. In der vorliegenden Untersuchung wurde der maximale 
Fang von C. pallidactylus in den Gelbschalen nach dem Erreichen einer Maximaltemperatur von $15{ }^{\circ} \mathrm{C}$ und einer mittleren Tagestemperatur von $9{ }^{\circ} \mathrm{C}$ ermittelt. Die Temperaturwerte für beide Ceutorhynchus-Arten entsprechen weitgehend den Angaben von DEBOUZIE \& BALLANGER (1993), JOHNEN \& MEIER (2000), JOHNEN (2001), BRosCHEWITZ (2001) und LindENBERG (2003).

Die eigene Untersuchung zeigte darüber hinaus, dass C. pallidactylus im Versuch 2001/02 in den Gelbschalen auf der Bodenoberfläche ab Ende März (ca. 20 Tage nach dem Zuflugsmaximum) in deutlich höheren Fangzahlen erfasst wurde als in den Gelbschalen in Bestandeshöhe. Dies ist möglicherweise mit der vertikalen Verteilung dieser Art im Bestand zu erklären. Nach BÜCHI (1988), KURRE (1997) und eigenen Untersuchungen befinden sich die meisten Eigelege des Gefleckten Kohltriebrüsslers in den unteren Blattetagen der Rapspflanzen. Die am Boden belassenen Gelbschalen werden von den sich bodennah aufhaltenden Käfern wahrscheinlich besser wahrgenommen und weisen daher höhere Fangzahlen auf. Außerdem kann das Fangergebnis der Gelbschalen am Boden auch dadurch erhöht worden sein, dass die Käfer zufällig von den Pflanzen in die Schalen gefallen waren. C. pallidactylus lässt sich nach eigenen Beobachtungen bereits bei geringfügigen Störungen zu Boden fallen. Auch FRITZSCHE (1957) stellte bei seinen Untersuchungen an Meligethes-Arten hohe Fangzahlen in den Bodenschalen fest, die er darauf zurückführte, dass ein Teil der Käfer durch die Bewegungen der Pflanzen passiv in die am Boden stehenden Schalen gelangte.

Das Geschlechterverhältnis von C. napi und C. pallidactylus in den Gelbschalenfängen war in diesem Versuch sehr stark zugunsten männlicher Tiere verschoben. Dies könnte zum einen mit einer unterschiedlichen Attraktivität der Gelbschalen für Männchen und Weibchen zusammenhängen. Zum anderen sind die Männchen bei ihrer Suche nach den Weibchen möglicherweise aktiver als die Weibchen.

\subsection{Einfluss der Pflanzendichte und -architektur auf die Befallsdichte von $P$. chryso- cephala}

Die vorliegenden Untersuchungen an P. chrysocephala zeigen, dass mit der Zunahme der Saatstärke bzw. der Pflanzendichte von Winterraps eine höhere Aktivitätsdichte der Imagines sowie eine höhere Ei- und Larvendichte pro $\mathrm{m}^{2}$ einhergeht. Eine Präferenz des Rapserdflohs für hohe Pflanzendichten stellte auch KAUFMANN (1941b) in einem Freilandversuch bei einer Saatmenge von $12 \mathrm{~kg} / \mathrm{ha}$ und einer Reihenentfernung von $40 \mathrm{~cm}$ fest. Dabei wurden unterschiedliche Pflanzendichten durch Vereinzelung der Pflanzen innerhalb der Reihen auf eine 
Entfernung von $2 \mathrm{~cm}, 4 \mathrm{~cm}$ und $8 \mathrm{~cm}$ erreicht; dies würde bei einer $100 \%$ igen Keimfähigkeit 125, 67 bzw. 31 Pflanzen $/ \mathrm{m}^{2}$ entsprechen. Seinen Ergebnissen zufolge fanden sich in den Parzellen mit den hohen Pflanzendichten mehr Käfer bzw. Larven pro $\mathrm{m}^{2}$ als auf den Flächen mit geringen Dichten.

Die Präferenz von $P$. chrysocephala für höhere Pflanzendichten erklärt sich wahrscheinlich aus den Temperatur- und Feuchtigkeitsansprüchen dieser Art. Die Imagines sind als Winterbrüter feuchtigkeits- und schattenliebend (KAUFMANN 1941b). Darüber hinaus benötigt der Rapserdfloh für die Entwicklung der Eier im Boden ausreichende Feuchtigkeit, da die Eier während der Embryonalentwicklung auf den Kontakt mit tropfbar flüssigem Wasser angewiesen sind (KAUFMANN 1941a, GODAN 1947, SchUlZ 1985). Nach KAUFMANN (1941a) werden die Eier nur in Mikrohabitate mit genügender Bodenfeuchte, die mit der sehr beweglichen Legescheide der Tiere ertastet werden, abgesetzt. Darüber hinaus ist auch anzunehmen, das die schlüpfenden Eilarven für ihre Wanderung zur Wirtspflanze eine ausreichende Bodenfeuchte benötigen. Untersuchungen von WAHMHOFF (2000) zur relativen Luftfeuchtigkeit in unterschiedlich dichten Rapsbeständen zeigten, dass der Rapserdfloh diese Bedingungen in den dichteren Pflanzenbeständen vermutlich besser verwirklicht findet als in den dünnen Beständen. Im Zeitraum von Mitte Oktober bis Mitte November war die durchschnittliche Luftfeuchtigkeit in den dichteren Rapsbeständen (100 Pfl. $\left./ \mathrm{m}^{2}\right)$ sowohl nachts als auch tags um 1 - $2 \%$ gegenüber dünnen Beständen (25 Pfl. $/ \mathrm{m}^{2}$ ) erhöht. Eine Bevorzugung von hohen Pflanzendichten mit einer höheren Luftfeuchtigkeit ist daher naheliegend.

Die höheren Dichten der Käfer pro $\mathrm{m}^{2}$ bei mittlerer $\left(60 \mathrm{Kö} . / \mathrm{m}^{2}\right)$ und hoher Pflanzendichte (90 $\mathrm{Kö.} / \mathrm{m}^{2}$ ) wurden allerdings nur in einem von zwei Versuchsjahren (2001) festgestellt. Im September 2000 hatten die Unterschiede in der Feuchtigkeit anders als im niederschlagsärmeren Oktober 2001 aufgrund überdurchschnittlich hoher Niederschläge im Zeitraum der Probenahme vermutlich keinen Einfluss auf die Käferdichten in den unterschiedlichen Saatvarianten. Auch bei der Pflanzendichte $30 \mathrm{Kö.} / \mathrm{m}^{2}$ dürfte in diesem Jahr eine ausreichend hohe Feuchtigkeit vorhanden gewesen sein. Dagegen hat möglicherweise das größere Angebot an Nahrungspflanzen und an Pflanzen für die Eiablage bei den hohen Pflanzendichten im Versuch $2000 \mathrm{zu}$ höheren Aktivitätsdichten geführt.

Ein weiterer Hinweis, der das Präferenzverhalten von $P$. chrysocephala erklären könnte, findet sich bei CIPOLLINI \& BERGELSON (2001). Die Autoren postulierten, dass das Abwehrvermögen der Rapspflanzen gegenüber herbivoren Insekten in dichten Beständen durch die intraspezifische Konkurrenz zwischen den Pflanzen herabgesetzt sein könnte. Sie stellten in ihren Untersuchungen fest, dass die geringere Nährstoffverfügbarkeit bei hohen Sommerrapsdichten eine 
Reduktion der Konzentration von Trypsininhibitoren in den Pflanzen bewirkte. Trypsininhibitoren stellen fraßinduzierte pflanzliche Abwehrstoffe dar, von denen in Laborversuchen gezeigt werden konnte, dass sie das Wachstum und die Überlebensdauer vieler Insektenarten verringern (CIPOLLINI \& BERGELSON 2001). Dies könnte die Attraktivität von Wirtspflanzen in hohen Bestandesdichten zusätzlich steigern.

Die höheren Ei- und Larvendichten pro $\mathrm{m}^{2}$ in Parzellen mit hoher Pflanzendichte sind nicht nur auf die höhere Pflanzenzahl pro $\mathrm{m}^{2}$, sondern auch auf die etwas höhere Eiablage des Rapserdflohs in größerer Entfernung von der Pflanze zurückzuführen. In Übereinstimmung mit den Befunden von KAUfMANN (1941a) legten die Weibchen von P. chrysocephala in beiden Versuchsjahren einen Teil der Eier auch in größeren Entfernungen von der Pflanze ab. Mit zunehmendem Abstand von der Pflanze war bei den beiden Pflanzendichten (30 und $60 \mathrm{Kö.} / \mathrm{m}^{2}$ ) im Versuch 2001 eine kontinuierliche Abnahme der Eidichten im Boden zu beobachten, die jedoch bei der geringen Pflanzendichte deutlich stärker ausfiel. Eine mögliche Ursache dafür könnte sein, dass die Transpiration von Bodenwasser in dichtstehenden Pflanzenbeständen aufgrund der Beschattung eingeschränkt ist, so dass die Bodenfeuchte auch in größerem Abstand von der Pflanze für die Eiablage ausreichte. In unmittelbarer Nähe der Pflanzen wurde in den Versuchen 2000 und 2001 unabhängig von der Pflanzendichte eine nahezu gleiche Anzahl Eier abgelegt. Eine genaue Analyse der Ursachen für dieses Verhalten der Erdflohweibchen muss Gegenstand weiterer Untersuchungen sein. Einige andere Schadinsekten passen ihr Eiablageverhalten stärker an die vorhandene Wirtspflanzendichte an. In Untersuchungen von DosDALL et al. (1996) zur Eiablage der Kohlfliege Delia spp. in Parzellen mit vergleichsweise sehr hohen Sommerrapsdichten $\left(95,183,434\right.$ und 763 Pfl. $\left./ \mathrm{m}^{2}\right)$ führte die Erhöhung der Pflanzendichte von 95 Pflanzen $/ \mathrm{m}^{2}$ auf 434 Pflanzen $/ \mathrm{m}^{2}$ zu einer Reduktion der Eizahl. Dies konnten die Autoren allerdings ebenfalls nur in einem von zwei Versuchsjahren feststellen.

Bezogen auf den Einzelpflanzenbefall wiesen die Pflanzen geringer Pflanzendichte in der vorliegenden Untersuchung eine deutlich höhere Larvenzahl pro Pflanze auf als die Pflanzen mittlerer und hoher Pflanzendichten. $\mathrm{Zu}$ einer ähnlichen Feststellung kam auch KAUFMANN (1941b) im Rahmen des Standweitenversuches, in dem mit zunehmender Vereinzelung der Pflanzen eine Zunahme der Larvenzahl pro Pflanze festgestellt wurde. MEUCHE (1940) und SCHULZ (1983) beobachteten in ihren Freilandversuchen ebenfalls den höchsten Larvenbefall von $P$. chrysocephala in großen und kräftigen Rapspflanzen mit weiterem Standraum.

In der Literatur findet sich eine Reihe von Hinweisen, nach denen herbivore Insekten bei der Eiablage systematisch kräftige und große Pflanzen als Wirtspflanze auswählen (GÜNTHART 1949, CARR et al. 1998). Im Gegensatz zu der von KAUFMANN (1944) und SCHULz (1983) ge- 
äußerten Vermutung spielte die Größe der Pflanzen in dieser Untersuchung keine Rolle bei der Wirtswahl der Weibchen. Das Merkmal Pflanzengröße hat möglicherweise bei Herbivoren, die wie die Triebschädlinge C. napi und C. pallidactylus ihre Eier direkt ins pflanzliche Gewebe ablegen, eine größere Bedeutung. Die Tatsache, dass die Eiablage des Rapserdflohs vorwiegend nachts und in den frühen Morgenstunden vonstatten geht (KAUFMANN 1941a), wenn der visuellen Sinneswahrnehmung eine geringere Bedeutung zukommt, spricht vielmehr für andere Faktoren.

Die bisherigen Ausführungen machen deutlich, dass die Verteilung des Rapserdflohs auf die Parzellen mit unterschiedlichen Pflanzendichten wahrscheinlich in erster Linie durch die Feuchtepräferenz der Imagines und Präimaginalstadien bestimmt wird. Die Reduktion des Einzelpflanzenbefalls in den Parzellen mit hohen Pflanzendichten ist darauf zurückzuführen, dass sich die bei hohen Pflanzendichten schlüpfenden Larven auf mehr Pflanzen in unmittelbarer Nähe verteilen konnten. In der geringen Pflanzendichte (30 Kö. $\left./ \mathrm{m}^{2}\right)$ kommt es hingegen zu einer stärkeren Konzentration der Larven auf die einzelne Pflanze, so dass der Larvenbefall pro Pflanze ansteigt.

\subsection{Einfluss der Pflanzendichte und -architektur auf die innerpflanzliche Verteilung und Schadwirkung von $P$. chrysocephala}

Die unterschiedlichen Pflanzendichten beeinflussten nicht nur die Entwicklung der Einzelpflanzenarchitektur (Kap. 3.1.4) und die Höhe des Einzelpflanzenbefalls (Kap. 3.2.5), sondern hatten auch Auswirkungen auf die innerpflanzliche Verteilung der Rapserdflohlarven. Diese steht wiederum in direkter Verbindung zur Schadwirkung von P. chrysocephala. Die Hauptschädigung des Rapserdflohs entsteht, wenn die Larven vor allem bei tiefen und wechselnden Temperaturen im Winter aus den Blattstielen in die Endknospe einwandern, dort den Vegetationspunkt zerstören und so zu einer Schwächung der Pflanzen oder zur parasitären Auswinterung führen (GODAN 1950, BUHL 1959).

Mit zunehmender Larvenzahl pro Pflanze erhöht sich die Wahrscheinlichkeit eines Endknospenbefalls deutlich. Nach ScHULZ (1983) können bei Linienraps mit einer Pflanzendichte von ca. 80 Pflanzen $/ \mathrm{m}^{2}$ und einem Befall von 5 bis 9 Larven pro Pflanze etwa $25 \%$ der Larven in den Endknospen auftreten. Bei 15 bis 19 Larven pro Pflanze erhöht sich der Endknospenbefall bereits auf $80 \%$. Die eigenen Untersuchungen mit der Linienrapssorte Mohican bestätigen dies. Während bei dieser Sorte im Versuch 1999/00 mit ansteigendem Einzelpflanzenbefall eine signifikante Zunahme des Endknospenbefalls festzustellen war, konnte bei der Hybrid- 
rapssorte Artus in den Versuchen 2000/01 und 2001/02 kein derartiger Zusammenhang nachgewiesen werden. Bei den wüchsigen Hybridrapssorten steigt die Wahrscheinlichkeit des schädlichen Endknospenbefalls mit zunehmendem Befallsniveau demnach nicht in gleichem Maße wie bei den Linienrapssorten.

Stirbt die Rapspflanze trotz des Endknospenbefalls nicht ab, können tiefer liegende Seitenknospen aktiviert werden (SCHULZ \& DAEBELER 1984) und den Verlust des Haupttriebes durch verstärkte Ausbildung von Seitentrieben teilweise kompensieren. Solche Pflanzen waren im Versuch 2000/01 in allen Saatstärkevarianten zu verzeichnen. Ein signifikanter Unterschied im Anteil der Pflanzen ohne Haupttrieb konnte jedoch zwischen den drei Versuchspflanzendichten nicht nachgewiesen werden.

In der vorliegenden Untersuchung war nur im einem Versuchsjahr ein Einfluss der Pflanzendichte auf den Befall der Endknospen und damit auf die Hauptschädigung des Rapserdflohes festzustellen (vgl. Kap. 4.1). Bei der geringen Bestandesdichte der wuchsstarken Hybridrapspflanzen war der Larvenbefall der Endknospen im Herbst des Versuches 2000/01 um den Faktor 3 geringer als bei den mittleren und hohen Pflanzendichten. Dies wurde vor allem durch die Verteilung der Larven auf eine wesentlich größere Anzahl stärker entwickelter Blätter und Seitentriebknospen in der geringen Saatstärke erreicht. Im darauffolgenden Winter kam es bei allen Pflanzendichten zu einem deutlich geringeren Endknospenbefall; gleichzeitig nahm der Befall der Seitentriebknospen bei allen Pflanzendichten drastisch zu. Dies wurde durch ein beschleunigtes Wachstum der jungen Blätter unterhalb der Endknospen, begünstigt durch kurze Wärmeperioden im Dezember, und durch die Umverteilung der Larven auf die heranwachsenden Seitentriebknospen möglich. GODAN (1950) stellte ebenfalls eine Minderung der Schadwirkung der Rapserdflohlarven fest, wenn das Wachstum der Pflanzen durch Wärmeperioden während des Winters gefördert wurde. Der Temperaturanstieg steigert nicht nur die Fraßaktivität der Larven, sondern auch das Wachstum der Pflanzen, so dass eine Kompensation erfolgen kann. Im Winter des 3. Versuchsjahres 2001/02 kam es hingegen zu einer leichten Erhöhung des Endknospenbefalls. Die verzögerte vorwinterliche Entwicklung der Pflanzen sowie anhaltend tiefe Wintertemperaturen hatten zur Folge, dass kein ausreichendes Angebot an Blättern und Seitentriebknospen vorhanden war.

Die Zerstörung der Endknospen und die damit verbundene Auswinterung der Pflanzen kann Auswirkungen auf den Ertrag haben. Wie der Vergleich zwischen Insektizid-behandelten und unbehandelten Parzellen zeigte, war das Niveau der Ertragseinbußen im Versuch 2000/01 jedoch sehr gering. Die geringste Ertragsminderung durch den Rapserdflohbefall war mit 1,3\% bei der Pflanzendichte $30 \mathrm{Kö.} / \mathrm{m}^{2}$ festzustellen. Bei den höheren Pflanzendichten lagen die 
Ertragseinbußen etwas höher (60 Kö./m²: 5,1 \%, 90 Kö./m²: 3,7 \%). Die Ertragsunterschiede waren nur bei der Pflanzendichte $60 \mathrm{Kö.} / \mathrm{m}^{2}$ signifikant.

In den unbehandelten Parzellen war über Winter bei der Pflanzendichte $90 \mathrm{Kö.} / \mathrm{m}^{2}$ ein um 8,0 \% höherer Anstieg der Pflanzenverluste als in den behandelten Teilflächen zu beobachten. Dennoch kam es nicht, wie in der mittleren Pflanzendichte, zu einem signifikanten Mehrertrag in den behandelten Parzellen. Dies beruhte vermutlich auf der trotz Insektizidbehandlung auch in den behandelten Parzellen eintretenden starken Reduktion der Pflanzenzahl über Winter, so dass sich die Differenz zwischen den vitalen Pflanzen in den behandelten und unbehandelten Parzellen verringerte.

Dieses Ergebnis macht deutlich, dass die kräftigen Pflanzen der geringen Pflanzendichte den Verlust der Haupttriebe durch Regeneration, d. h. durch Mobilisierung von Seitentriebknospen, im Vergleich zu den höheren Pflanzendichten am besten kompensieren konnten.

Für die Schadwirkung des Rapserdflohes ist neben dem Befall der empfindlichen Endknospen auch das Verhältnis zwischen der Biomasse und dem Larvenbefall der Pflanzen von Bedeutung. In Übereinstimmung mit den Ergebnissen von ScHULZ (1983) hat die eigene Untersuchung im Herbst des Versuches 2001/02 ergeben, dass die größeren Pflanzen der geringen Pflanzendichte $\left(30 \mathrm{Kö.} / \mathrm{m}^{2}\right.$ ) bezogen auf ihre Biomasse einen deutlich geringeren Larvenbefall aufwiesen als die kleinen Pflanzen der hohen Pflanzendichte $\left(60 \mathrm{Kö.} / \mathrm{m}^{2}\right)$. Im Februar 2001 und Januar 2002 war die Larvenzahl/g Biomasse in allen Varianten etwa gleich, d. h. trotz der Blattverluste im Winter und eines absolut höheren Einzelpflanzenbefalls stand den Larven in den Pflanzen der geringen Bestandesdichte $\left(30 \mathrm{Kö.} / \mathrm{m}^{2}\right)$ eine etwa ebenso große Biomasse pro Larve zur Verfügung wie in den Pflanzen der mittleren und hohen Bestandesdichten (60 und $90 \mathrm{Kö.} / \mathrm{m}^{2}$ ). Rapspflanzen mit mehr oberirdischer Biomasse können im Herbst größere Assimilatreserven in die Wurzeln einlagern, so dass das Regenerationsvermögen der Rapspflanze sich deutlich erhöht (HENNING 1979). Sie können vermutlich die durch den Minierfraß der Larven entstandene Schädigung besser kompensieren als Pflanzen mit einer geringeren Biobzw. Wurzelmasse. Abnehmende Pflanzendichten erhöhen also die Toleranz der Pflanzen gegenüber dem Rapserdflohbefall, was hinsichtlich der Ertragsstabilität der Pflanzen von entscheidender Bedeutung ist.

Für die Bewertung der Schadwirkung des Rapserdflohs ist auch die Anzahl der Larven von Bedeutung, die eine Pflanze ohne Ertragseinbußen tolerieren kann. Als Bekämpfungsschwelle für den Larvenbefall werden 3 bis 5 Larven pro Pflanze angegeben (GODAN 1950, BUHL 1959, Hoßfeld 1993, LAUENSTEIN 1993). In Untersuchungen von Schulz (1983 und mdl. Mitt.) an Linienraps mit einer Pflanzendichte von ca. 80 Pflanzen $/ \mathrm{m}^{2}$ traten bei einem Befall von 3 bis 7 
Larven pro Pflanze noch keine Ertragsverluste auf. In den eigenen Versuchen der Jahre 2000/01 und 2001/02 tolerierten die kräftigen Hybridrapspflanzen bei geringer Pflanzendichte (30 Kö./m²) sogar einen Larvenbefall von 16,4 bzw. 11,9 Larven pro Pflanze ohne Ertragseinbußen. Im Versuch 2000/01 kam es bei den Pflanzen der hohen Pflanzendichte $\left(90 \mathrm{Kö} . / \mathrm{m}^{2}\right)$ bei einem Larvenbefall von 6,4 Larven je Pflanze nicht zu einem signifikanten Ertragsabfall, wohingegen in der mittleren Pflanzendichte $\left(60 \mathrm{Kö.} / \mathrm{m}^{2}\right)$ ein Larvenbefall von 10,3 Larven je Pflanze zu einer Ertragsdepression von 5,1 \% führte. Der höhere Ertragsabfall von 11,1 \% bei einem Befall von 8,1 Larven je Pflanze in der Variante $60 \mathrm{Kö.} / \mathrm{m}^{2}$ im Versuch 2001/02 ist auf die verzögerte vorwinterliche Entwicklung der Pflanzen zurückzuführen.

Aus den vorgestellten Ergebnissen können erste Ansätze für eine Anpassung der Bekämpfungsschwelle für die Rapserdflohlarven bei Anbau von Hybridraps mit reduzierten Saatstärken abgeleitet werden. Da ein Larvenbefall von 12 - 16 Larven je Pflanze bei geringer Pflanzendichte $\left(30 \mathrm{Kö.} / \mathrm{m}^{2}\right)$ des Hybridrapses noch keine Ertragsdepression hervorrief, ist zu erwägen den Schwellenwert von 3 - 5 Larven pro Pflanze auf eine mindestens doppelt so hohe Larvenzahl je Pflanze heraufzusetzen. Um dies genauer bestimmen zu können, sind jedoch weitere Untersuchungen notwendig. Einschränkend muss festgestellt werden, dass die Kultur Raps von einigen weiteren Schadinsekten besiedelt wird, deren Wirkungen auf den Ertrag in dieser Untersuchung nicht berücksichtigt werden konnte (vgl. LERIN 1995). Darüber hinaus bestehen enge Wechselbeziehungen zwischen dem Befall des Winterrapses mit P. chrysocephala und der Wurzelhals- und Stängelfäule Leptosphaeria maculans (Desm.) Ces. \& de Not. (Nebenfruchtform: Phoma lingam Tode ex Fries) (Broschewitz \& DAEbeler 1987, Steinbach 1990, Broschewitz et al. 1993, WAHMHOFF 2000). Nach Schulz \& DAEBELER (1984) können die Larven des Rapserdflohs einen 2,5-fach höheren Phoma-Befall an Blattstielen und Trieben induzieren. Die Ertragsverluste beliefen sich in den befallenen Parzellen im dreijährigen Mittel auf etwa 15 \%. Auch in späteren Untersuchungen fand SCHULZ (1993) einen deutlich stärkeren Phoma-Befall der Pflanzen, deren Blattstiele von Rapserdflohlarven befallen waren. Die Wurzelhals- und Stängelfäule hat ihrerseits Auswirkungen auf den Ertrag der Winterrapskulturen (WILLIAMS et al. 1999, HOWLETT et al. 2001) und überlagert die Schadwirkung der Triebschädlinge. 


\subsection{Einfluss der Pflanzendichte und -architektur auf die Befallsdichte von $C$. napi und C. pallidactylus}

Insgesamt waren die Befallsstärken von C. napi und C. pallidactylus in den Untersuchungsjahren auf einem ungewöhnlich hohen Niveau. Zwischen den drei Jahren zeigten sich jedoch bei beiden Arten deutliche Unterschiede. Diese Unterschiede waren insbesondere auf die Stärke der Besiedlung in Abhängigkeit vom Standort (Nähe zum Winterlager), den Witterungsverlauf während der Eiablagephase und die Pflanzenarchitektur zurückzuführen. Bei C. napi war die Larvenabundanz pro $\mathrm{m}^{2}$ im Jahr 2002 dreimal so hoch wie in den Jahren 2000 und 2001, bei C. pallidactylus dagegen im Jahr 2000 etwa doppelt so hoch wie in den Jahren 2001 und 2002 (Tab. A-6, Anhang). Die unmittelbare Nähe der Versuchsfläche zum Überwinterungsort (Wald) des Kohltriebrüsslers hatte im Jahr 2000 vermutlich eine starke Zuwanderung der Käfer zur Folge. Bei dem Rapsstängelrüssler führte offenbar die spät einsetzende starke Verzweigung der Pflanzen im Versuch 2002 zu einem optimalen Angebot an Eiablageorten.

Aus den Ergebnissen der vorliegenden Untersuchung geht hervor, dass die Pflanzen der geringen Pflanzendichte eine höhere Befallsstärke mit Eigelegen und Larven von C. pallidactylus aufwiesen als die der hohen Pflanzendichten. Bei C. napi war nur im Versuch 2000/01 mit abnehmender Pflanzendichte ein signifikanter Anstieg der Befallsdichte pro Pflanze zu beobachten. Bezogen auf die Dichte der Eigelege und Larven pro $\mathrm{m}^{2}$ wiesen C. napi und C. pallidactylus jedoch keine signifikanten Unterschiede zwischen den Pflanzendichten auf.

Nach BroschewiTz (1985) werden dichte Winterrapsbestände (100 Pfl. $\left./ \mathrm{m}^{2}\right)$ stärker durch $C$. pallidactylus befallen, weil sie den Weibchen mehr Ressourcen für die Eiablage bieten. Diese Angaben beziehen sich auf den Anteil befallener Rapspflanzen; er stieg mit zunehmender Pflanzendichte an. BENEDEK (1984) gibt ebenfalls an, dass der Befall durch C. pallidactylus in dichten Winterrapsbeständen weitaus höher ist als in dünnen Beständen. In Untersuchungen von FINCH \& SKINNER (1976) in Blumenkohlparzellen mit 1,5 bis 82,6 Pflanzen $/ \mathrm{m}^{2}$ war die Dichte der Larven von C. pallidactylus pro $\mathrm{m}^{2}$ bei den hohen Pflanzendichten höher als bei den geringen Pflanzendichten; der Befall der Einzelpflanzen unterschied sich dabei nicht. Die Kulturen Kohl und Raps sind in Bezug auf ihr Potenzial an Eiablagemöglichkeiten jedoch nicht direkt vergleichbar. Im Gegensatz zu der starken Verzweigung von Raps bei der geringen Pflanzendichte und der wesentlich höheren Anzahl von Blättern an Haupt- und Seitentrieben treten bei dem Blumenkohl keine Seitentriebe auf. Bei den stärker verzweigten Winterrapspflanzen der geringen Pflanzendichte waren auch bei hohem Befallsniveau von C. pallidactylus genügend Eiablageressourcen vorhanden, um den Einzelpflanzenbefall gegenüber den hohen Pflanzendichten deutlich zu erhöhen. 
Der höhere Ei- und Larvenbefall der Einzelpflanzen in den Parzellen mit geringer Pflanzendichte entspricht recht gut den theoretisch erwarteten Befallsunterschieden. Unter der Voraussetzung, dass die Weibchen in allen Varianten die gleiche Dichte aufwiesen und sie ihren gesamten Eivorrat bei geringer Pflanzendichte auf entsprechend weniger Pflanzen verteilen als bei hoher Bestandesdichte, müsste eine Halbierung der Pflanzendichte theoretisch den Befall pro Einzelpflanze verdoppeln. Wie die eigenen Ergebnisse zeigen, kam es in den Jahren 2000, 2001 und 2002 bei der Pflanzendichte $30 \mathrm{Kö.} / \mathrm{m}^{2}$ zu einem 1,7-, 1,4- bzw. 2,5-fach höheren Einzelpflanzenbefall von C. pallidactylus als bei der Pflanzendichte $60 \mathrm{Kö.} / \mathrm{m}^{2}$. Im Vergleich zu der Pflanzendichte $90 \mathrm{Kö.} / \mathrm{m}^{2} \mathrm{kam}$ es bei der Dichte $30 \mathrm{Kö.} / \mathrm{m}^{2}$ in den Jahren 2000 und $2001 \mathrm{zu}$ einem 2,2- bzw. 3,0-fach höheren Einzelpflanzenbefall. Die Abweichungen von der theoretisch zu erwartenden Verdoppelung bzw. Verdreifachung des Einzelpflanzenbefalls bei der Pflanzendichte $30 \mathrm{Kö.} / \mathrm{m}^{2}$ sind wahrscheinlich auf das von Jahr zu Jahr variierende Angebot an Eiablageressourcen sowie die unterschiedliche Eimortalität und Konkurrenz zwischen den Weibchen um Eiablageplätze in den einzelnen Jahren zurückzuführen. Auch die Pflanzendichten wichen von der mit der Saatstärke angestrebten Relation von $1: 2: 3$ in den Saatvarianten $\mathrm{ab}$.

Die größeren Pflanzen der geringen Bestandesdichte könnten aufgrund ihrer besseren Wirtspflanzenqualität für die Larvenentwicklung bevorzugt werden. Nach CARR et al. (1998) bevorzugt die Blattwespe Nematus oligospilus (Hym.: Tenthredinidae) bei der Eiablage gezielt längere Triebe der Weidenart Salix lasiolepis, was sowohl bei den Larven als auch bei der Folgegeneration zu einer Steigerung der Fitness führt. Der höhere Herbivorenbefall auf großen Pflanzen führt zudem häufig zu einem verminderten Prädationsrisiko für das einzelne Individuum (HAMiLTON 1971, BEGON et al. 1991). Hinzu kommt noch, dass die Weibchen bei der Eiablage möglicherweise längere Zeit auf den größeren Pflanzen mit mehr Blättern verbringen, wohingegen sie in einem dichten Pflanzenbestand häufiger auf andere Pflanzen überwechseln.

Da der Larvenbefall von C. pallidactylus pro $\mathrm{m}^{2}$ in der vorliegenden Untersuchung bei allen Pflanzendichten annähernd gleich war, spricht dies für eine zufällige Verteilung der Käfer über alle Versuchsparzellen. Auch GÜNTHART (1949) stellte bei Untersuchungen an Blumenkohl fest, dass sich die Imagines von $C$. pallidactylus unabhängig von der Pflanzengröße auf der Fläche zufällig verteilten. Die zufällige Verteilung der Käfer und Eigelege im Bestand könnte auf einer Strategie zur Risikoverminderung basieren. Die Risikominimierung ist ein wichtiger Faktor bei dem Eiablageverhalten vieler phytophager Insekten (ROOT \& KAREIVA 1984). Das langsame Wachstum und die geringe Mobilität der frühen Entwicklungsstadien 
vieler Herbivoren verknüpft deren Schicksal eng mit dem ihrer Wirtspflanze. Umwelteinflüsse, die die Vitalität der einzelnen Pflanze beeinflussen, wirken sich auch unmittelbar auf die Fitness der Herbivoren aus. Die Verteilung der Eier auf möglichst viele Wirtspflanzen wirkt diesen Risiken entgegen (RoOT \& KAREIVA 1984, SHEA et al. 2000).

Aus der vorliegenden Untersuchung lässt sich ableiten, dass C. pallidactylus bei der Eiablage keine Präferenz für bestimmte Pflanzendichten aufweist. Die Weibchen verteilen ihre Eier auf die Blätter der vorhandenen Haupt- und Seitentriebe. Der unterschiedliche Larvenbefall pro Pflanze ist vorrangig auf die Unterschiede in der Pflanzenzahl zurückzuführen.

Die Eiablage von C. napi wird im Gegensatz zu C. pallidactylus wesentlich durch die Pflanzenarchitektur beeinflusst. Nach JOURDHEUIL (1961) und BÜCHI (1996) ist die Eiablage von $C$. napi in die Haupttriebe ab einer Pflanzenhöhe von ca. $22 \mathrm{~cm}$ erheblich vermindert. BÜCHI (1996) führt dies auf die Präferenz der Weibchen für junges Gewebe zurück. Die meisten Eier des Rapsstängelrüsslers sind daher unmittelbar unter den Triebspitzen lokalisiert, wo sich junges und zartes Gewebe befindet (Meuche 1942, GÜNTHART 1949, DEUBERT 1955). Neben den Haupttrieben werden von $C$. napi auch die unteren markreichen Seitentriebe mit Eiern belegt (MeUChe 1942, GÜNTHART 1949, KURRE 1997). In den eigenen Untersuchungen hat sich gezeigt, dass die Eiablage von C. napi zusätzlich durch den Durchmesser der Haupttriebe beeinflusst wird. In einer gesonderten Pflanzenprobe am 2. April 2002 fanden sich in den Pflanzen mit dem größeren Stängelbasisdurchmesser $\left(\mathrm{X}_{\mathrm{m}} \pm \mathrm{SD}, \varnothing: 17,3 \pm 2,0 \mathrm{~mm}\right.$, Trieblänge: $28,0 \pm$ 3,9 cm) im Mittel 8,4 \pm 4,1 Eier, in den Pflanzen mit dem geringen Stängelbasisdurchmesser ( $\varnothing: 9,2 \pm 1,1 \mathrm{~mm}$, Trieblänge 23,9 $\pm 3,0 \mathrm{~cm}$ ) dagegen nur 2,0 $\pm 2,3$ Eier.

Wie auch bei C. pallidactylus kam es durch die Reduzierung der Pflanzendichte von 60 zu 30 Kö./m² in den Jahren 2000, 2001 und 2002 zu einem 1,3-, 1,9- bzw. 1,6-fachen Anstieg der Larvenzahl/Pflanze von C. napi. Im Vergleich zu der Pflanzendichte $90 \mathrm{Kö.} / \mathrm{m}^{2} \mathrm{kam}$ es bei der Dichte $30 \mathrm{Kö.} / \mathrm{m}^{2}$ in den Jahren 2000 und $2001 \mathrm{zu}$ einem 1,3- bzw. 3,9-fach höheren Einzelpflanzenbefall. Auch hier waren die Abweichungen von den theoretisch erwarteten Relationen vermutlich durch die unterschiedlichen Pflanzendichten, Rapssorten und unterschiedlichen Mortalitätsfaktoren der Imagines, Eier und Larven bedingt.

Der höhere Befall der Pflanzen mit C. napi bei der geringen Pflanzendichte im Vergleich zu den beiden hohen Pflanzendichten kann im Versuch 2000/01 durch die Wüchsigkeit der Hybridrapsorte, die bessere Entwicklung der Pflanzen vor dem Winter und den früheren Beginn des Längenwachstums im Frühjahr erklärt werden. Dies führte insgesamt im Vergleich zu den anderen Versuchsjahren zu einem früheren Angebot der Eiablageressourcen in den Haupttrie- 
ben (s. Tab. A-4, Anhang). In der gesonderten Pflanzenprobe konnte gezeigt werden, dass $C$. napi zu Beginn der Eiablage kräftige Rapspflanzen mit größerem Stängelbasisdurchmesser bevorzugt, wie sie für die geringe Pflanzendichte charakteristisch sind (s. Kap. 3.3.10.4). Zu ähnlichen Feststellungen für einzeln stehende Rapspflanzen kamen auch GoDAN (1950) und WAHMHOFF (2000). Gleichzeitig mindert ein großer Stängeldurchmesser den Prädationsdruck durch Parasitoide. Nach NitZSCHE (1998) sinkt die Parasitierung der Larven von C. napi mit zunehmendem Stängeldurchmesser, da die maximale Einstichtiefe des Ovipositors der Weibchen von Tersilochus fulvipes (Hym.: Tersilochinae) auf ca. $4 \mathrm{~mm}$ begrenzt ist. Außerdem hatten die günstigen vorwinterlichen Wachstumsbedingungen der wuchsstarken Hybridsorte bei geringer Pflanzendichte im Versuch 2000/01 eine frühzeitige Ausbildung geeigneter Seitentriebe zur Folge, die den Weibchen für einen längeren Zeitraum gute Eiablagemöglichkeiten boten. Da die Eiablage von C. napi in die Haupttriebe ab einer Höhe von $22 \mathrm{~cm}$ stark reduziert ist (BÜCHI 1996), konzentriert sich die weitere Eiablage weitgehend auf die Seitentriebe. Kleinere Seitentriebe mit einem Triebdurchmesser unter 1,7 mm wurden noch nicht mit Eiern belegt. Die gesamte Eiablageperiode wurde so offenbar verlängert, so dass die Anzahl der Eigelege pro Pflanze erhöht war. Die stärkere Wüchsigkeit der Haupttriebe und die frühzeitige Ausbildung von Seitentrieben bei der Hybridrapssorte beeinflussten vor allem die Pflanzen der geringen Bestandesdichte, die aufgrund ihres größeren Standraumes über das größere Verzweigungspotenzial verfügten.

In den Versuchsjahren 1999/00 und 2001/02 konnte im Einzelpflanzenbefall von C. napi kein signifikanter Unterschied zwischen den Saatstärkevarianten festgestellt werden. Bei der Linienrapssorte im Versuch 1999/00 wurden die Seitentriebe erst relativ spät ausgebildet, so dass sie für die Eiablage von C. napi auch bei geringer Pflanzendichte ohne Bedeutung blieben. Bei der im Versuch 2001/02 angebauten Hybridrapssorte kam es aufgrund der verhaltenen vorwinterlichen Entwicklung der Pflanzen zu einer erst später einsetzenden, verstärkten Ausbildung der Seitentriebe. Dies ermöglichte eine bis in die 3. Maidekade anhaltende Eiablage in die Seitentriebe, insbesondere bei der geringen Pflanzendichte (s. Kap. 3.3.10.2, Abb. 50). Die hohe Schwankungsbreite der Einzelwerte in der geringen Pflanzendichte $\left(30 \mathrm{Kö.} / \mathrm{m}^{2}:\right.$ 0,3 bis 10,3 Eier und Larven pro Pflanze und Seitentrieb) hat jedoch eine statistische Absicherung in diesem Jahr verhindert.

Die bei allen Saatstärken nahezu gleiche Befallsdichte von C. napi pro Flächeneinheit deutet darauf hin, dass die geringere Anzahl der Haupttriebe in der Saatvariante $30 \mathrm{Kö} . / \mathrm{m}^{2}$ durch die entsprechend höhere Anzahl von Seitentrieben, die für die Eiablage zur Verfügung standen, kompensiert wurde. 
Die Reduktion der Pflanzendichte kann also den Einzelpflanzenbefall von C. napi in Abhängigkeit von der Wüchsigkeit und dem Verzweigungspotenzial der Rapssorte sowie der vorwinterlichen Entwicklung der Pflanzen in unterschiedlichem Ausmaß fördern. Der unterschiedliche Einzelpflanzenbefall der beiden Ceutorhynchus-Arten lässt sich durch die Eiablage in die unterschiedlichen Pflanzenorgane und deren Verfügbarkeit und Eignung erklären.

\subsection{Einfluss der Pflanzendichte und -architektur auf die innerpflanzliche Verteilung und Schadwirkung von $C$. napi und $C$. pallidactylus}

Die durch die Pflanzendichte und Rapssorte hervorgerufenen Unterschiede in der phänologischen und morphologischen Entwicklung der Rapspflanzen hatten eine unterschiedliche Verteilung der Eigelege und Larven von C. napi und C. pallidactylus in den Trieben und Blättern zur Folge. Die Verteilung der Eigelege von C. napi und C. pallidactylus auf die Haupt- und Seitentriebe war stark von der Rapssorte abhängig. Bei der Hybridrapssorte hatten die Eigelege beider Schädlingsarten in den Seitentrieben einen deutlich höheren Anteil am Gesamtbefall als bei der Linienrapssorte. Dies beruhte auf dem höheren Verzweigungspotential der Hybridrapssorte und somit einem größeren Angebot an Eiablagemöglichkeiten.

Anhand der Verteilung der älteren Larvenstadien kann auf die zeitliche Abfolge der Eiablage in verschiedene Organe der Pflanzen geschlossen werden. Der höhere Anteil der L3-Larven von C. napi in den Seitentrieben der geringen Pflanzendichte weist darauf hin, dass die Seitentriebe dieser Variante früher für die Eiablage zur Verfügung standen. Auch bei C. pallidactylus standen im ersten und dritten Versuchsjahr die Blätter der Seitentriebe bei geringer Pflanzendichte früher für die Eiablage zur Verfügung als bei höheren Pflanzendichten. Im 2. Versuchsjahr kam es unmittelbar nach dem Zuflug zu einer zweiwöchigen Kälteperiode, die vermutlich die Eiablage von C. pallidactylus verzögert hat. Ein um das 3-fache höherer Anteil der L3-Larven in den Haupttrieben der mittleren und hohen Pflanzendichte im Vergleich zur geringen Pflanzendichte deutet darauf hin, dass die dichteren Bestände in diesem Jahr zunächst bevorzugt wurden. Mit ansteigenden Temperaturen und fortschreitender Eiablage wurden die Pflanzen der geringen Bestandesdichte von C. pallidactylus verstärkt mit Eigelegen versehen, was an dem hohen Befallsniveau mit den L1- und L2-Larven in den Seitentrieben zu erkennen ist.

Die Verteilung der Eigelege von C. pallidactylus auf die Blätter und Triebe der Rapspflanzen zeigte darüber hinaus, dass neben den Blättern an den Haupt- und Seitentrieben auch die Triebe selbst mit Eiern belegt werden können. Im Versuchsjahr 1999/00 wurde ein vergleichswei- 
se hoher Anteil von Eigelegen unter die Epidermis der Haupttriebe in den mittleren und hohen Pflanzendichte abgelegt (s. Kap. 3.3.9.3, Abb. 37). Dies liegt wahrscheinlich darin begründet, dass die in diesem Jahr verwendete Linienrapssorte insbesondere bei höheren Bestandesdichten deutlich kleinere und damit für die Eiablage günstigere Pflanzenstängel aufwies als die wuchsstarke Hybridrapssorte in den übrigen Jahren. Die dünnen Haupttriebe der Linienrapssorte waren in diesem Jahr möglicherweise für die Eiablage besser geeignet als die kräftigeren Triebe der Linien- und der Hybridrapssorte in den anderen Jahren.

Die Verteilung der Eigelege von C. pallidactylus auf die einzelnen Blattetagen und verschiedenen Blattbereiche (apikales, mittleres und basales Drittel) erklärt sich aus der unterschiedlichen Größe der Blätter in den verschiedenen Pflanzendichten. Bei der geringen Pflanzendichte waren in allen Jahren deutlich größere Blätter zu beobachten, die aufgrund ihrer Blattmorphologie zusätzliche Eiablageressourcen boten. Während bei den kleineren Blättern vorwiegend die Blattstiele für die Eiablage genutzt wurden, konnten bei den kräftiger entwickelten Blättern auch der an den Blattstiel angrenzende Bereich der basalen Blattfiedern und der Blattspreitenbasis sowie die Verzweigungen der Blattadern verstärkt mit Eiern belegt werden. Die Weibchen von C. pallidactylus sind demnach in der Lage, auf die sich ändernde Pflanzendichte sehr plastisch zu reagieren, in dem sie alle ihnen zur Verfügung stehenden Eiablageorte an den Pflanzen ausschöpfen. Sie passen dabei die Größe ihrer Eigelege den vorhandenen Eiablagemöglichkeiten an, indem sie in größeren und kräftigen Blättern häufiger kleinere Eigelege absetzen als in kleinen Blättern. Da die größeren Blätter häufig eine höhere Anzahl von Eigelegen aufweisen, könnte dies als Reaktion der Weibchen zur Vermeidung von intraspezifischer Konkurrenz zwischen den Larven gedeutet werden.

Da im Frühjahr alle Parzellen ohne Insektizidbehandlung blieben, erlauben die in der vorliegenden Untersuchung erhobenen Ertragsdaten keine genauen quantitativen Aussagen zum Einfluss unterschiedlicher Pflanzendichten auf die Schadwirkung von C. napi und C. pallidactylus. Das besonders hohe Befallsniveau der Larven beider Schadinsekten im Versuch 2001/02 könnte jedoch durchaus zu dem vergleichsweise geringen Kornertrag in diesem Versuch beigetragen haben.

Neben $P$. chrysocephala gehört $C$. napi in Mitteleuropa zu den wirtschaftlich bedeutendsten Schadinsektenarten im Winterraps (Еквом 1995). Die Eiablage von C. napi löst an den Pflanzen gallenartige Wucherungen, Stängelverkrümmungen und Stängelstauchungen aus; insbesondere bei einem frühen Beginn der Eiablage in noch kleine Pflanzen und wüchsigem Wetter nach der Eiablage platzt der Stängel an den Eiablagestellen häufig auf (GÜNTHART 1949, DOSSE 1951, JOHNEN 2001). Die Ertragsminderung durch C. napi kann nach BALLANGER (1987) in 
den humiden Regionen Mittelfrankreichs bis zu $40 \%$ und in den trockeneren Regionen Südfrankreichs bis zu $80 \%$ betragen.

Das Schadenspotenzial von C. pallidactylus wird in der Literatur vergleichsweise gering eingestuft (KöRTing 1942, Broschewitz 1985, Broschewitz \& DAeBeler 1987, Winfield 1992, GARBE et al. 1996). Nach KELM et al. (1995) ist die Schadwirkung von C. pallidactylus insbesondere in den niederschlagsreichen Jahren gering. Die Wundkallusbildung der Pflanze im Bereich der Eiablagestellen wird durch die Feuchtigkeit während der Eiablageperiode gefördert (KAZDA 1956). Nach BROSCHEWITZ \& DAEBELER (1987) werden ca. 40 \% der abgelegten Eier, nach PALOSZ (1980) sogar bis zu 70 \% der Eier von C. pallidactylus durch die Wundkallusbildung zerstört. In vorliegender Untersuchung wurden auf diese Weise ebenfalls Verluste der Eigelege in Höhe von 36 \% (2002) bzw. 76 \% (2000) festgestellt. Dieser Unterschied ist jedoch eher auf den Termin der Untersuchung als auf die Feuchtigkeit in diesen Jahren zurückzuführen. Ein Einfluss der Pflanzendichte auf diese Abwehrreaktion der Pflanze konnte hierbei nicht nachgewiesen werden. Das feuchte Wetter trägt weiterhin dazu bei, dass die Pflanzen die Schadwirkung der C. pallidactylus-Larven besser durch Wachstum kompensieren können (KELM \& KLUKOWSKI 2000). In den klimatisch ungünstigen, trockeneren Anbaugebieten in Polen wird C. pallidactylus daher ein erheblich größerer Einfluss auf den Ertrag zugeschrieben als in feuchten Lagen. In Abhängigkeit von der Niederschlagsmenge können sich die Ertragsverluste durch C. pallidactylus bis auf $32 \%$ belaufen (KELM \& KLUKOWSKI 2000). Demzufolge dürften die überdurchschnittlich hohen Niederschläge im Versuch 2001/02 die Kompensationsfähigkeit des Hybridrapses gestärkt und ein noch weiteres Absinken des Kornertrages verhindert haben.

Zur Kompensation der Schadwirkung von C. napi und C. pallidactylus in kräftiger entwickelten Rapspflanzen liegen in der Literatur zahlreiche Hinweise vor. WAHMHOFF (2000) beurteilte die Schädigung der Rapspflanzen anhand der Ausdehnung der durch die Larven im Stängelmark verursachten Fraßminen; dabei hat er allerdings nicht zwischen den drei im Stängel minierenden Triebschädlingsarten ( $P$. chrysocephala, C. napi, C. pallidactylus) unterschieden. Er stellte fest, dass trotz eines höheren Larvenbefalls der Einzelpflanzen im Ausmaß der Fraßminen nur geringe Unterschiede zwischen den Pflanzendichten (25, 50 und $\left.100 \mathrm{Pfl} . / \mathrm{m}^{2}\right)$ vorlagen. Dies führt der Autor auf die größeren Stängeldurchmesser der Pflanzen in den niedrigen Bestandesdichten zurück: In den dickeren Stängeln mit großem Markvolumen wurde ein geringerer Anteil des Marks gefressen als in dünnen Stängeln. Somit stieg das Ausmaß des Schadens in kräftigen Pflanzen trotz höheren Larvenbefalls/Pflanze nicht entsprechend an. $\mathrm{Zu}$ einem ähnlichen Schluss kam auch BROSCHEWITZ (1985) bei seinen Untersuchungen zur 
Schadwirkung von C. pallidactylus. Der Anteil des Fraßgangs am Durchmesser des Stängels war bei dünnen Winterrapspflanzen (Triebdurchmesser 6 bis $7 \mathrm{~mm}$ ) mit 54 bis $71 \%$ deutlich höher als bei dicken Pflanzen (Triebdurchmesser 10 bis $13 \mathrm{~mm}$ ) mit 35 bis $49 \%$. PALOSZ (1980) fand bei C. pallidactylus in schwach entwickelten Rapspflanzen mit weniger als 4 Seitentrieben eine negative Beziehung zwischen dem Befallsgrad und dem mittleren Kornertrag pro Schote. Ähnliche Beobachtungen wurden auch von BROSCHEWITZ (1985) gemacht. Bei einer Pflanzendichte von 100 Pflanzen $/ \mathrm{m}^{2}$ zur Ernte erzielten die mit C. pallidactylus befallenen Rapspflanzen nur etwa 90 \% des Ertrages der Pflanzen ohne Befall. Demgegenüber konnten kräftige Rapspflanzen, die sich bei Pflanzendichten von 50 bis 80 Pflanzen $/ \mathrm{m}^{2}$ entwickeln, einen Befall von 14 bis 20 C. pallidactylus-Larven pro Pflanze ohne Ertragseinbußen tolerieren (BRosChewitz \& DAEBELER 1987). Die Schadwirkung von C. pallidactylus ist demzufolge bei den kräftigen Pflanzen der geringen Pflanzendichten erheblich geringer einzuschätzen.

Neben dem Kornertrag werden auch die Standfestigkeit des Winterrapses sowie der Befall der Pflanzen mit Leptosphaeria maculans durch die Fraßaktivität der Larven von C. napi und $C$. pallidactylus beeinflusst. Nach BROSCHEWITZ \& DAEBELER (1987) weisen Rapspflanzen hoher Bestandesdichten bei Befall mit Larven von C. pallidactylus eine geringere Standfestigkeit auf als Pflanzen mittlerer Bestandesdichten, ohne aber genauere Angaben zu diesen Bestandesdichten zu machen. Die fördernde Wirkung von C. pallidactylus auf den Befall der Pflanzen mit Leptosphaeria maculans ist geringer einzuschätzen als die von C. napi, der dem Pilz aufgrund seines Schadbildes (Aufplatzen des Stängels im Bereich der Eigelege) großflächige Infektionsmöglichkeiten bietet (BROSCHEWITZ 1985, BROSCHEWITZ \& DAEBELER 1987). Nach DAebeler et al. (1981) ist die Ausbreitung des Phoma-Befalls im Stängel umso stärker, je geringer der Stängeldurchmesser ist. Dies verdeutlicht, dass schwächere Rapspflanzen, wie sie bei höheren Bestandesdichten gegeben sind, bei einem kombinierten Befall mit den Larven der Triebschädlinge und Leptosphaeria maculans stärker gefährdet sind als kräftige Pflanzen. In den eigenen Versuchen trat kein Lager auf, der Phoma-Befall der Stängel wurde nicht erfasst.

\subsection{Einfluss der Pflanzendichte und -architektur auf den Kornertrag}

In allen drei Versuchsjahren hatte die Pflanzendichte trotz der großen Abstände zwischen den Dichtestufen (30, 60 und $\left.90 \mathrm{Kö.} / \mathrm{m}^{2}\right)$ keinen Einfluss auf den Kornertrag und das Tausendkorngewicht der Winterrapsparzellen. Der Ertrag pro Einzelpflanze nahm mit abnehmender Pflanzendichte ungeachtet des höheren Schädlingsbefalls signifikant zu. 
Zahlreiche Untersuchungen zum Einfluss der Bestandesdichte auf den Kornertrag im Rapsanbau führten zu dem Ergebnis, dass die Pflanzenzahl $/ \mathrm{m}^{2}$ in einem weiten Bereich unterschiedlicher Pflanzendichten kaum Auswirkungen auf den Kornertrag hat. SCARISBRICK et al. (1982) konnten im einem Bereich von 24 bis 126 Pflanzen $/ \mathrm{m}^{2}$ keinen Einfluss auf den Flächenertrag feststellen. Auch mehrjährige Untersuchungen von BoELKE (1981) ergaben, dass bei einer großen Bandbreite von 30 bis 100 Pflanzen $/ \mathrm{m}^{2}$ vergleichbare Höchstertrage zu erzielen sind. SIERTS (1987) fand bei Bestandesdichten von 40 bis 80 Pflanzen $/ \mathrm{m}^{2}$ ähnlich hohe Kornerträge. In Untersuchungen von GeISLER \& STOY (1987), in denen Pflanzendichten von 10 bis 185 Pflanzen $/ \mathrm{m}^{2}$ verglichen wurden, wurde mit 45 Pflanzen $/ \mathrm{m}^{2}$ im Herbst, entsprechend 43 Pflanzen $/ \mathrm{m}^{2}$ im Frühjahr, bereits der höchste Flächenertrag erreicht. Die Obergrenze der Frühjahrsbestandesdichte, bei der noch ein vergleichbarer Flächenertrag erzielt wurde, lag bei 80 Pflanzen $/ \mathrm{m}^{2}$. Die hier dargestellten Ergebnisse verschiedener Autoren beziehen sich auf Linienrapssorten. Bei den neuerdings verstärkt angebauten Hybridrapssorten, die auch in der vorliegenden Arbeit einbezogen wurden, sind Spitzenerträge schon mit Pflanzendichten von 30 bis 40 Pflanzen $/ \mathrm{m}^{2}$ bei der Ernte möglich (DiEPENBRock 1999, Aigner 2000). Bei früher Saat und günstigen Feldaufgangs- und Wachstumsbedingungen wird bei Hybridraps daher eine Saatstärke von 35 bis 40 Körnern/m² empfohlen (DENNERT \& FISCHBECK 2000, SAUERMANN \& GRONOW 2000). Nach neueren Ergebnissen von AIGNER (2003) konnte weder bei den Hybridsorten noch bei den Linienrapssorten durch die Reduktion der Aussaatstärke auf $31 \mathrm{Körner} / \mathrm{m}^{2}$ ein wirtschaftlicher Ertragsrückgang festgestellt werden. Bereits ein Winterrapsbestand mit 20 ausreichend über die Fläche verteilten, gesunden und kräftigen Pflanzen pro $\mathrm{m}^{2}$ zur Ernte kann Spitzenerträge erzielen. Die eigenen Ergebnisse zum Kornertrag in den Saatvarianten stimmen mit diesen Literaturangaben überein.

Eine naheliegende Erklärung für den geringen Einfluss der Pflanzendichte auf den Ertrag besteht darin, dass die Rapspflanze in ihrer Ertragsbildung in einem weiten Bereich von Pflanzendichten sehr plastisch auf den Standraum reagiert (GEISLER \& STOY 1987, McGREGOR 1987). Der Rapsertrag setzt sich aus den Komponenten Pflanzen $/ \mathrm{m}^{2}$, Anzahl Schoten pro Pflanze (bestehend aus Anzahl schotentragender Sprossachsen pro Pflanze und Schoten pro Sprossachse), Anzahl Samen pro Schote und Einzelsamengewicht zusammen (GEISLER \& HENNING 1981a, DiePENBROCK et al. 1999). Während das Samengewicht sowie die Anzahl Samen/Schote Komponenten des Ertrages darstellen, die kaum auf Umwelteinflüssen reagieren, sind die Anzahl der Seitentriebe und die Schotenzahl pro Seitentrieb stark von den Wachstumsbedingungen im Bestand abhängig (GEISLER \& STOY 1987, AigNER 2003). Bei einer Reduktion der Saatstärke wird der Ertrag der Rapspflanze daher wesentlich durch die 
Zahl der Seitentriebe und die Schotenzahl bestimmt (MCGregor 1987, Aigner 2003). Mit abnehmender Pflanzendichte und verminderter Standraumkonkurrenz der Rapspflanzen werden die Ertragsparameter Anzahl der Seitentriebe, Schotenzahl/Pflanze und Einzelpflanzenertrag gefördert (HenNing 1979, Stoy 1983, Geisler \& StOy 1987, MCGREGOR 1987, SiERTS et al. 1987, МомOH \& ZHOU 2001, AigNER 2003). Auch in der vorliegenden Untersuchung war die Anzahl der Seitentriebe und der Einzelpflanzenertrag mit abnehmender Bestandesdichte signifikant erhöht. In den geringen Pflanzendichten entfällt ein größerer Anteil der Gesamtschotenzahl auf die Seitentriebe des basalen Sprossbereiches, während bei hohem Konkurrenzdruck in dichten Beständen die Haupt- und Seitentriebe des oberen Sprossbereiches den größten Teil der Gesamtschotenmenge stellen (HENNING 1979, BoELKE 1981). Eine genauere Analyse der Einflüsse der Triebschädlinge auf die einzelnen Ertragskomponenten konnte im Rahmen dieser Arbeit nicht vorgenommen werden, da alle Parzellen im Frühjahr ohne Insektizidbehandlung blieben. Nach LERIN (1988) vermindert der Befall durch C. napi die Ertragsleistung des Haupttriebes durch eine geringfügige Reduktion der Schotenzahl und der Schotenproduktivität. OPPERMANN (1990) ermittelte in einem Käfigversuch bei einer Dichte von 2 Imagines von C. napi je $\mathrm{m}^{2}$ einen Pflanzenbefall von 12,5 \% und eine Reduktion der Schotenzahl um 7,6 \%. Darüber hinaus kommt es zu einer vermehrten Mobilisierung von Seitentrieben (SCHRIMPF 1954, LERIN 1988). Die Ergebnisse der vorliegenden Untersuchung lassen erkennen, dass auch nach einem starken Befall durch $P$. chrysocephala eine Mobilisierung von Seitentrieben erfolgt. Bei C. pallidactylus fanden KELM \& WALCZAK (1998) einen positiven Zusammenhang zwischen der Länge der Miniergänge der Larven in den Haupt- und Seitentrieben und deren Schotenansatz.

Das Samengewicht ist grundsätzlich von der Position der Schote abhängig. Die höchsten Samengewichte finden sich in den Schoten am Haupttrieb, während an den Seitentrieben 1. bzw. 2. Ordnung geringere Samengewichte vorliegen (GEISLER \& HENNING 1981b). Ein signifikanter Einfluss auf das Tausendkorngewicht konnte in dieser Arbeit wie auch in den anderen Untersuchungen (GEISLER \& HENNING 1981b, SCARISBRICK et al. 1982, AignER 2003) nicht nachgewiesen werden. Dies war vermutlich auf die sich mit der Bestandesdichte ändernden Standraumzumessungen der Rapspflanzen zurückzuführen.

Die vorliegende Untersuchung wie auch die Befunde anderer Autoren bestätigen die große Anpassungsfähigkeit der Rapspflanze, die durch die Variation der Anzahl Verzweigungen und Schoten in der Lage ist, vorhandene Standräume für den Ertragsaufbau auszunutzen (BOELKE 1981, AIGNER 2003). 


\section{Zusammenfassung}

In den Anbaujahren 1999/00 bis 2001/02 wurden im Raum Göttingen Untersuchungen zum Einfluss der Pflanzendichte und -architektur auf die Abundanz und innerpflanzliche Verteilung der Triebschädlinge $P$. chrysocephala, C. napi und C. pallidactylus in Winterraps durchgeführt. Dazu wurden in den drei Jahren Parzellenversuche mit verschiedenen Saatstärken (30, 60 und $90 \mathrm{Kö.} / \mathrm{m}^{2}$; 2001/02: 30 und $60 \mathrm{Kö.} / \mathrm{m}^{2}$ ) und je 6 Wiederholungen angelegt. Im Versuch 1999/00 wurde die Linienrapssorte Mohican, in den Versuchen 2000/01 und 2001/02 die Hybridrapssorte Artus angebaut. Mit Hilfe von Gelbschalen wurden der Beginn der Aktivität von $C$. napi auf den Überwinterungsfeldern und die Zuwanderung der drei Triebschädlinge in die Versuchsfelder erfasst. Die Aktivitätsdichte und Abundanz der Imagines von P. chrysocephala wurden mit Fangschalen und Photoeklektoren, die Eidichten durch Flotation aus Bodenproben bestimmt. Die Abundanz und räumlich-zeitliche Verteilung der Larven von $P$. chrysocephala und der Eigelege und Larven von C. napi und C. pallidactylus in den Pflanzen sowie die pflanzenmorphologischen Parameter wurden jeweils von Oktober bis Mai an Pflanzenproben ermittelt.

Die verschiedenen Saatstärken führten in allen Versuchsjahren zu entsprechend abgestuften, signifikant verschiedenen Bestandesdichten: Die Saatstärke $30 \mathrm{Kö.} / \mathrm{m}^{2}$ hatte gegenüber den Saatstärken 60 und $90 \mathrm{Kö.} / \mathrm{m}^{2}$ eine jeweils signifikant geringere Pflanzendichte und wesentlich kräftiger entwickelte Einzelpflanzen zur Folge. Mit zunehmender Saatstärke sanken Anzahl der Blätter und Seitentriebe, Pflanzenlänge, Sprossdurchmesser am Wurzelhals und in $50 \mathrm{~cm}$ Höhe, Länge und Durchmesser der Seitentriebe, Anzahl schotentragender Seitentriebe sowie Biomasseproduktion der Rapspflanzen signifikant ab.

Die Imagines von $P$. chrysocephala zeigten in einem von zwei Versuchen eine statistisch gesicherte Präferenz für hohe Rapsdichten. Ihre Aktivitätsdichte war bei geringer Pflanzendichte deutlich niedriger als bei den hohen Pflanzendichten. Die Eidichte von P. chrysocephala pro $\mathrm{m}^{2}$ stieg im Versuch 2001/02 mit der Pflanzendichte an. Mit zunehmendem Abstand von der Pflanze sank die Eidichte bei geringer Pflanzendichte stärker ab als bei hoher Pflanzendichte. Die Larvenzahl pro $\mathrm{m}^{2}$ stieg in allen Versuchen mit der Pflanzendichte an; sie unterschied sich bei den verschiedenen Pflanzendichten jedoch nur im Versuch 2001/02 signifikant. Die Larvenzahl pro Pflanze war in der geringen Pflanzendichte gegenüber den hohen Pflanzendichten 
signifikant erhöht; bezogen auf Gramm Biomasse der Pflanzen glich sich dieser Unterschied allerdings aus.

Die räumlich-zeitliche Verteilung der Larven von P. chrysocephala auf die einzelnen Blattetagen der Rapspflanzen zeigte, dass die Larven mit fortschreitender Besiedlung in den schwach entwickelten Pflanzen früher die jüngsten Blätter unter der Endknospe erreichten als in den kräftigen Pflanzen. Ein Einfluss der Pflanzendichte auf den Befall der Endknospen war nur im Versuch 2000/01 bei der Hybridrapssorte Artus zu beobachten. Während der Endknospenbefall bei der geringen Pflanzendichte durch die Verteilung der Larven auf eine erheblich größere Anzahl gut entwickelter Blätter und Seitentriebknospen vermindert war, stieg er bei den beiden hohen Pflanzendichten signifikant an. Zwischen der Abundanz der Larven von P. chrysocephala in den Pflanzen und der Abundanz der Larven in den Endknospen zeigte sich nur bei der Linienrapssorte Mohican ein signifikanter, positiver Zusammenhang. Bei der Hybridrapssorte stieg der für die Schadwirkung bedeutende Endknospenbefall dagegen mit zunehmender Larvenzahl nur in geringem Maße an.

Ein signifikanter Einfluss der chemischen Bekämpfung der Larven von P. chrysocephala auf den Kornertrag war nur bei der Saatvariante $60 \mathrm{Kö.} / \mathrm{m}^{2}$ in den Versuchen 2000/01 und 2001/02 festzustellen: Ein Larvenbefall von 10,3 bzw. 8,1 Larven je Pflanze führte zu einer Ertragsdepression von 5,1 \% bzw. 11,1 \%. Der höhere Ertragsabfall im Versuch 2001/02 wurde auf die verzögerte vorwinterliche Entwicklung der Pflanzen zurückgeführt. Die kräftigen Hybridrapspflanzen der geringen Pflanzendichte $\left(30 \mathrm{Kö.} / \mathrm{m}^{2}\right)$ tolerierten einen Larvenbefall von $16,4 \mathrm{bzw}$. 11,9 Larven je Pflanze ohne Ertragseinbußen. Bei den Pflanzen der hohen Pflanzendichte (90 Kö. $/ \mathrm{m}^{2}$ ) kam es im Versuch 2000/01 bei einem Larvenbefall von 6,4 Larven je Pflanze nicht zu einem Ertragsabfall.

Die Abundanz der Eigelege und Larven von C. pallidactylus pro Pflanze war in allen Versuchen bei geringer Pflanzendichte höher als bei den hohen Pflanzendichten. Bei C. napi war nur im Versuch 2000/01 mit abnehmender Pflanzendichte ein signifikanter Anstieg der Befallsdichte pro Pflanze zu beobachten. Bezogen auf die Abundanz der Eigelege und Larven pro $\mathrm{m}^{2}$ zeigten C. napi und C. pallidactylus in allen Versuchen keine signifikanten Unterschiede zwischen den Pflanzendichten. Demnach wiesen beide Ceutorhynchus-Arten bei der Eiablage keine Präferenz für bestimmte Pflanzendichten auf.

Die durch die Pflanzendichte hervorgerufenen Unterschiede in der phänologischen und morphologischen Entwicklung der Rapspflanzen hatten eine unterschiedliche Verteilung der Eigelege und Larven von C. napi und C. pallidactylus in Trieben und Blättern zur Folge; dies ist 
auf die Eiablagepräferenz der Weibchen für bestimmte Pflanzenorgane sowie deren Verfügbarkeit und Entwicklungsstand zurückzuführen. Die Weibchen von C. napi verteilten ihre Eier auf die Haupt- und Seitentriebe, wobei Seitentriebe erst ab einem Triebdurchmesser von 1,7 mm mit Eiern belegt wurden. Die Weibchen von C. pallidactylus legten ihre Eier in die Blätter an Haupt- und Seitentrieben sowie zu einem geringen Anteil in die Triebe selbst ab.

Die Verteilung der Eigelege von C. napi und C. pallidactylus auf die Haupt- und Seitentriebe bzw. deren Blätter war stark von der Rapssorte abhängig. Bei der Hybridrapssorte, die eine frühere und stärkere Verzweigung aufwies, hatten die Eigelege beider Arten in den Seitentrieben einen deutlich höheren Anteil am Gesamtbefall als bei der Linienrapssorte. In einer gesonderten Pflanzenprobe wurde gezeigt, dass C. napi zu Beginn der Eiablage kräftige Rapspflanzen mit größerem Stängelbasisdurchmesser bevorzugt, wie sie für die geringe Pflanzendichte charakteristisch sind.

Die unterschiedliche Verteilung der Eigelege von C. pallidactylus auf die einzelnen Blattetagen und verschiedenen Blattbereiche (apikales, mittleres und basales Drittel) wurden mit der unterschiedlichen Größe der Blätter in den verschiedenen Pflanzendichten erklärt. Die Blätter der geringen Pflanzendichte boten aufgrund ihrer Blattmorphologie zusätzliche Eiablageressourcen. Die Weibchen von C. pallidactylus waren in der Lage, auf die sich ändernde Pflanzendichte sehr plastisch zu reagieren, indem sie die zur Verfügung stehenden Eiablageorte an den Pflanzen ausschöpften. Sie passten dabei die Größe ihrer Eigelege den Eiablageressourcen an, indem sie in größere und kräftige Blätter häufiger kleinere Eigelege absetzten als in kleine Blätter. Da die größeren Blätter eine höhere Anzahl von Eigelegen aufwiesen, ist dies als Strategie zur Verminderung intraspezifischer Konkurrenz zwischen den Larven zu deuten.

Zwischen dem Kornertrag/ha und dem Tausendkorngewicht in verschiedenen Pflanzendichten konnten keine signifikanten Unterschiede festgestellt werden. Der Ertrag pro Einzelpflanze nahm mit abnehmender Pflanzendichte trotz höheren Schädlingsbefalls signifikant zu. Dies wurde auf das große Kompensationsvermögen der Rapspflanze, die durch die Anpassung der Anzahl Verzweigungen und Schoten in der Lage ist, auch höhere Befallsdichten ohne Ertragsminderung zu tolerieren, zurückgeführt.

Die Ergebnisse dieser Untersuchung zeigen insgesamt, dass das Risiko der Pflanzenschäden durch die untersuchten Triebschädlinge bei einer Reduzierung der Pflanzendichte nicht erhöht ist; unter günstigen Wachstumsbedingungen können die Pflanzen bei geringen Pflanzendichten die Schäden besser kompensieren. 


\section{Literaturverzeichnis}

Aigner, A. (2000): Bayern: Hybridsorten vor dem Durchbruch? - Top Agrar 7, 64-65.

Aigner, A. (2003): Kann die Aussaatstärke bei Winterraps weiter gesenkt werden? - Schule und Beratung 6, Seiten III-1 - III-5.

Alford, D. V., Nilsson, C. \& Ulber, B. (2003): 2. Insects pests of oilseed rape crops. - In: ALFORD, D. V. (Hrsg.): Biocontrol of oilseed rape pests. - Blackwell Verlag, Berlin, 941 .

Asjakin, B. P. (1986): [Die Besonderheit des Verhaltens der Kohlfliege in Bezug auf die Spezialisierung und Zentralisierung im Gemüseanbau. - Arbeiten der entomologischen Gesellschaft der Sowjetunion.] Bd. 68. Ak. Wiss. UdSSR, Leningrad, 165-169.

Auld, D. L., BetTis, B. L. \& DiAL, M. J. (1984): Planting date and cultivar effect on winter rape production. - Agron. J. 76, 197-200.

BALlanger, Y. (1987): Raisonnement de la lutte contre le gros Charancon de la tige du colza (Ceutorrhynchus napi Gyll.) - IOBC wprs Bull. 10, 79-84.

BASKY, Z. \& HOPPER, K. R. (2000): Impact of plant density and natural enemy exclosure on abundance of Duraphis noxia (KURDJUMOv) and Rhopalosiphum padi (L.) (Hom., Aphididae) in Hungary. - J. Appl. Entomol. 124, 99-103.

Begon, M., Harper, J. L., Townsend, C. R. (1991): Ökologie - Individuen, Populationen und Lebensgemeinschaften. - 1. Aufl., Birkhäuser Verlag, Basel, Boston, Berlin, 1024 S.

BENEDEK, P. (1984): Moderner Pflanzenschutz beim Rapsanbau. - Internat. Z. Landwirtsch. 6, 524-527.

BoElCKe, B. (1981): Untersuchungen zur optimalen Bestandesdichte bei Winterraps. - Arch. Acker- u. Pflanzenbau u. Bodenkd. 25, 637-643.

Broschewitz, B. (1985): Untersuchungen zur Biologie und Schadwirkung des Gefleckten Kohltriebrüßlers (Ceutorhynchus quadridens Panzer) am Winterraps (Brassica napus L. var. oleifera Metzg.). - Diss. Rostock, 149 S.

Broschewitz, B. (2001): Vorblüteschädlinge im Winterraps. Überwachung und Schadwirkung. - Raps 19, 8-12.

Broschewitz, B. \& DAebeler, F. (1987): Beitrag zur Biologie und Schadwirkung des Gefleckten Kohltriebrüßlers (Ceutorhynchus quadridens Panz.) am Winterraps. Nachrichtenbl. Pflanzenschutz DDR 41, 34-37. 
Broschewitz, B., Stenbach, P. \& Goltermann, S. (1993): Einfluss stengelbewohnender tierischer Schaderreger auf den Befall von Winterraps mit Phoma lingam und Botrytis cinerea. - Ges. Pflanzen 45, 106-110.

BÜCHI, R. (1988): Neue Bekämpfungsschwelle für den Rapsstengelrüssler Ceutorhynchus napi Gyll. - Mitt. Schweiz. Landw. 36, 110-117.

BÜCHI, R. (1996): Eiablage des Rapsstengelrüßlers Ceutorhynchus napi Gyll. in Abhängigkeit der Stengellänge bei verschiedenen Rapssorten. - Anz. Schädlingskde., Pflanzenschutz, Umweltschutz 69, 136-139.

BÜCHS, W. (1997): Gefleckten Kohltriebrüßler und Großen Rapsstengelrüßler gezielt bekämpfen. - Raps 15, 39-41.

BuHL, K. (1959): Beobachtungen und Untersuchungen über Biologie und Bekämpfung des Rapserdflohs (Psylliodes chrysocephala L.) in Schleswig-Holstein. - Z. Pflanzenkrankh. (Pflanzenpath.) Pflanzenschutz 66, 6, 321-338.

CARr, T. G., RoIninen, H. \& Price, P. W. (1998): Oviposition preference and larval performance of Nematus oligospilus (Hymenoptera: Tenthredinidae) in relation to host plant vigor. - Environ. Entomol. 27, 615-625.

Cipollini, D. F. \& Bergelson, J. (2001): Plant density and nutrient availability constrain constitutive and wound-induced expression of trypsin inhibitors in Brassica napus. - J. Chem. Ecol. 27, 593-610.

Clarke, J. M. \& Simpson, G. M. (1978): Influence of irrigation and seeding rates on yield and yield components of Brassica napus cv. Tower. - Can. J. Plant Sci. 58, 731-737.

Cloyd, R. A. \& SAdOF, C. S. (2000): Effects of plant architecture on the attack rate of Leptomastix dactylopii (Hymenoptera: Encyrtidae) a parasitoid of the citrus mealybug (Homoptera: Pseudococcidae). - Environ. Entomol. 29, 535-541.

Costa-Arbulú, C. \& SÁnchez, A. M. (2000): Does patch density of Gnaphalium robustum PHIL. influence herbivory by Helicoverpa zea (BODDIE) larvae? - Rev. Chil. Entomol. 26, 69-71.

Cromartie, W. J. (1975) : The effect of stand size and vegetational background on the colonisation of cruciferous plants by herbivorous insects. - J. Appl. Ecol. 12, 517-533.

DAebeler, F., Amelung, D. \& SeIDEl, D. (1981): Die wichtigsten pilzparasitären Krankheiten des Rapses und Möglichkeiten zu ihrer Einschränkung. - Nachr.-Bl. Pflanzenschutz DDR 35, 249-251.

Debouzie, D. \& Ballanger, Y. (1993): Dynamics of a Ceutorhynchus napi population in winter rape fields. - Acta Oecol. 14, 603-618. 
DenNeRT, J. \& FISCHBECK, G. (2000): Anbaumanagement von Winterraps. - Raps 18, 106110.

DeuberT, K.-H. (1955): Beiträge zu den Beziehungen zwischen Ceuthorrhynchus napi Gyll. (Col. Curc.) und Winterraps hinsichtlich der Gallenbildung mit Ovarienuntersuchungen an verschiedenen Ceuthorrhynchus-Arten. - Wiss. Z. Univ. Halle, Math.-Nat. 4, 909932.

DiEPENBROCK, W. (1999): Das Ertragspotential von Winterraps. - Raps 17, 166-169.

Diepenbrock, W., Fischbeck, G., Heyland, K.-U. \& Knauer, N. (1999): Spezieller Pflanzenbau. - 3. Aufl., Ulmer Verlag, Stuttgart, 523 S.

Dмосн, J. (1958): Wstepne obserwacje nad biologia chowaczy: czterozebnego Ceuthorrhynchus quadridens Panz. i podobnika C. assimilis Payk. - Polskie Pismo Entomologiczne, Seria B, Zeszyt 1-2, 15-20, Wroclaw.

Dмосн, J. (1959): Badania nad chowaczem czterozebnym Ceuthorrhynchus quadridens Panz. (Morfologia, biologia, ekologia oraz znaczenie dla rzepaku). - Prace Naukowe Instytutu Ochrony Roslin, Tom I, Zeszyt 3, 37-74, Warszawa.

Dosdall, L. M., Herbut, M. J., Cowle, N. T. \& Micklich, T. M. (1996): The effect of seeding date and plant density on infestations of root maggots, Delia spp. (Diptera: Anthomyiidae), in canola. - Can. J. Plant Sci. 76, 169-177.

Dosse, G. (1951): Der Große Kohltriebrüßler Ceutorhynchus napi (Gyll.). Biologie, Schadauftreten und Bekämpfung unter besonderer Berücksichtigung der „Gallbildung“ an Kohlpflanzen. - Z. angew. Entomol. 32, 489-566.

Dosse, G. (1953): Zur Biologie und Morphologie des Schwarzen Triebrüßlers (Ceutorhynchus picitarsis Gyll.) mit differential-diagnostischen Angaben zur Untersuchung der Larven von C. napi Gyll., C. quadridens Panz. und C. picitarsis Gyll.. - Z. angew. Entomol. 34, 303-312.

EbBe-Nymann, E. (1952): Rapsjordloppan Psylliodes chrysocephala L. Bidrag till kännedom om dess biologi och bekämpning. - Statens Växtskyddsanst. Medd. 63, 103 S., Ref. Z. Pflanzenkrankh. 61 (1954), 36.

Ekbom, B. (1995): Insect Pests. - In: Kimber, D., McGregor, R. (Hrsg.): Brassica oilseeds: Production and utilization. - CAB International, Wallingford, 141-152.

Finch, S. \& SKInNER, G. (1976): The effect of plant density on populations of the cabbage root fly (Erioischia brassicae (Bch.)) and the cabbage stem weevil (Ceutorhynchus quadridens (Panz.)) on cauliflowers. - Bull. ent. Res. 66, 113-123. 
Freude, H., Harde, K. W. \& Lohse, G. A. (1983): Die Käfer Mitteleuropas, Bd. 11, Verlag Goecke \& Evers, Krefeld, 342 S.

FRITZSCHE, R. (1956): Untersuchungen zur Bekämpfung der Rapsschädlinge IV: Beitrag zur Ökologie und Bekämpfung des Großen Rapsstengelrüßlers (Ceuthorrhynchus napi Gyll.). - Nachrichtenbl. Dt. Pflanzenschutzdienst (Berlin) NF 10, 97-105.

FRITZSCHE, R. (1957): Zur Biologie und Ökologie der Rapsschädlinge aus der Gattung Meligethes. - Z. angew. Entomol. 40, 222-280.

Furlan, F. (1991): Schädlingskontrolle im Körnerrapsanbau. - Der Pflanzenarzt 44, 9-10.

Garbe, V., Broschewitz, B., Erichsen, E., Hoßfeld, R., Lauenstein, G., Steinbach, P., Ulber, B. \& Zellner, M. (1996): Schadensschwellen bei Rapsschädlingen. - Raps 14, 58-63.

Geisler, G. (1981): Die Bestandesdichte beim Raps. - Bauernbl. Schleswig-Holstein 28, 5256.

GeIsLeR, G. \& HenNING, K. (1981a): Untersuchungen zur Ertragsstruktur von Raps (Brassica napus L. var. napus). I: Die vegetative Entwicklung der Rapspflanze in Abhängigkeit von der Bestandesdichte. - Bayer. Landw. Jb. 58, 203-211.

GeISLER, G. \& HENNING, K. (1981b): Untersuchungen zur Ertragsstruktur von Raps (Brassica napus L. var. napus). II: Die generative Entwicklung der Rapspflanze in Abhängigkeit von der Bestandesdichte. - Bayer. Landw. Jb. 58, 322-332.

GeISLER, G. \& Stoy, A. (1987): Untersuchungen zum Einfluß der Bestandesdichte auf das Ertragspotential von Rapspflanzen (Brassica napus L. var. napus). - J. Agron. Crop Sci. 159, 232-240.

Godan, D. (1947): Der Einfluss der Witterung auf den Massenwechsel des Rapserdflohs (Psylliodes chrysocephala L.). - Nachrichtenbl. Dt. Pflanzenschutzdienst. (Berlin) NF 1, 101-104.

GoDAN, D. (1948): Über Prognosestellungen, betreffend Massenvermehrungen von Raps- und Rübsenschädlingen. - Nachrichtenbl. Dt. Pflanzenschutzdienst. (Berlin) NF 2, 148152.

GodAN, D. (1950): Über die Wirkung des Rapserdflohlarvenbefalls auf die Rapspflanze. Mitt. Biol. Zentralanst. Land.- u. Forstwirtsch. 69, 1-35.

GÜNTHART, E. (1949): Beiträge zur Lebensweise und Bekämpfung von Ceutorrhynchus quadridens Panz. und Ceutorrhynchus napi Gyll. mit Beobachtungen an weiteren Kohl- und Rapschädlingen. - Mitt. Schweiz. Entomol. Ges. 22, 441-591.

Hamilton, W. D. (1971): Geometry for the selfish herd. - J. Theor. Biol. 31, 295-311. 
HASKEN, K.-H. (2001): Der Einfluß von Extensivierung auf das Auftreten von Getreideblattläusen und ihren natürlichen Gegenspielern im Winterweizen. - Diss. Univ. Göttingen, $121 \mathrm{~S}$.

HENNING, K. (1979): Untersuchungen über die Entwicklung und Ertragsbildung zweier Winterrapssorten unter besonderer Berücksichtigung des Einflusses von Bestandesdichte und Saatzeit. - Diss. Univ. Kiel, 154 S.

HenNING, K. (1983): Die Bedeutung der optimalen Standraumausnutzung bei Winterraps erste Erkenntnisse über die Einzelkornsaat. - Proc. 6th Int. Rapeseed Conf., Paris, 694698.

HonÉK, A. (1979): Plant density and occurrence of Coccinella septempunctata and Propylaea quatuordecimpunctata (Coleoptera, Coccinellidae) in cereals. - Acta entomol. Bohem. 75, 308-312.

HONÉK, A. (1983): Factors affecting the distribution of larvae of aphid predators (Col., Coccinellidae and Dipt. Syrphidae) in cereal stands. - Z. angew. Entomol. 95, 336-343.

HonÉK, A. (1985): Plant density and abundance of cereal aphids (Hom. Aphidina). - Z. angew. Entomol. 100, 399-409.

HONÉK, A. (1988): The effect of crop density and microclimate on pitfall trap catches of Carabidae, Staphylinidae (Coleoptera) and Lycosidae (Aranae) in cereal fields. - Pedobiologia 32, 233-242.

HonÉK, A. \& JARosík, V. (2000): The role of crop density, seed and aphid presence in diversification of field communities of Carabidae (Coleoptera). - Eur. J. Entomol. 97, 517525.

HonÉk, A. \& MARTinkova, Z. (1999): Host-plant mediated influences on development of Sitobion avenae (Sternorrhyncha: Aphididae). - Eur. J. Entomol. 96, 135-141.

HonÉk, A. \& MARTinkova, Z. (2004): The effect of environmentally induced variation of host-plant vigour on abundance of cereal aphids. - In: Simon, J.-C., DeDRYver, C.-A., RisPe, C. \& Hullé, M. (Hrsg.): Aphids in a new millennium, INRA, Paris, 319-324.

Honék, A., Martinkova, Z. \& LipavskÁ, H. (1998): Distribution of Metopolophium dirhodum in maize and cereals. - In: Nieto Nafria, J. M. \& Dixon, A. F. G. (Hrsg.): Aphids in natural and managed ecosystems, Universidad de León, León, 569-578.

HoßFELD, R. (1993): Die Gelbschale als Entscheidungshilfe bei der Bekämpfung des Rapserdflohs (Psylliodes chrysocephala L.). - Ges. Pflanzen 45, 291-295.

Howlett, B. J., Idnurm, A. \& Pedras, M. S. C. (2001): Leptosphaeria maculans, the causal agent of blackleg disease of Brassicas. - Fungal Genet. Biol. 33, 1-14. 
JoHnEN, A. (2001): Optimale Schädlingskontrolle im Frühjahr. Entscheidungsgrundlagen und Bekämpfungsstrategien. - Raps 19, 14-18.

Johnen, A. \& MeIER, H. (2000): A weather-based decision support system for managing oilseed rape pests. - BCPC Conference Pests \& Diseases, Brighton, 13-16.

Jourdheuil, P. (1961): Recherches récentes sur le charancon de la tige du colza, Ceuthorhynchus napi Gyll. - Oléagineux 6, 389-395.

Kaufmann, O. (1941a): Zur Biologie des Rapserdflohes (Psylliodes chrysocephala L.). - Z. PflKrankh. (Pflanzenpath.) PflSchutz 51, 305-324.

Kaufmann, O. (1941b): Epidemiologie und Massenwechsel des Rapserdflohs (Psylliodes chrysocephala L.). - Z. Pflanzenkrankh. (Pflanzenpath.) Pflanzenschutz 51, 342-369.

Kaufmann, O. (1944): Zur Epidemiologie und Bekämpfung des Rapserdflohs (Psylliodes chrysocephala L.). - Z. Pflanzenkrankh. (Pflanzenpath.) Pflanzenschutz 54, 257-276.

KAZDA, V. (1956): Die in der Tschechoslowakei den Kohl- und Rapspflanzen schädlichen Rüsselkäfer, unter besonderer Berücksichtigung des Ceuthorrhynchus napi Gyll. Mededeelingen der Landbouwhogeschool en der opzoekingsstations van den Staat te Gent 21, 411-420.

Kelm, M. \& KLUKOwsKi, Z. (2000): Weather as a factor determining damage caused by oilseed rape pests. - IOBC wprs Bull. 23, 119-124.

Kelm, M., WalczaK, B. \& Williams, I. H. (1995): Changes in growth and yield parameters of winter rape as a result of injury by the stem weevil (Ceutorhynchus quadridens). Proc. $9^{\text {th }}$ Int. Rapeseed Congress Cambridge UK, 4 - 7 Juli, 673-675.

Koubaiti, K., Lerin, J. (1992): Fecundity and egg laying dynamics of Baris coerulescens Scop. (Col., Curculionidae) on oilseed rape. - J. Appl. Entomol. 114, 289-297.

KÖRTING, A. (1942): Über die Lebensweise des gefleckten Kohltriebrüßlers (Ceutorhynchus quadridens Panz.) und seine Bedeutung als Ölfruchtschädling. - Arb. physiol. angew. Ent. 9, 207-237.

KURRE, H.-H. (1997): Untersuchung zur Eiablage, Larvenentwicklung und Schadwirkung des Großen Rapsstengelrüßlers (Ceutorhynchus napi Gyll.) und Gefleckten Kohltriebrüßlers (Ceutorhynchus quadridens Mrsh.) bei getrenntem und kombiniertem Befall von Rapspflanzen. - Diplomarbeit Univ. Göttingen, 84 S.

LAMB, R. J. (1989): Entomology of oilseed Brassica crops. - Ann. Rev. Entomol. 34, 211 229.

LAuEnSteIn, G. (1993): Schwellenwerte für die Bekämpfung von Rapsschädlingen. - Raps 11, 27-31. 
LINDENBERG, H. (2003): Vorblüteschädlinge in Winterraps. Großer Rapsstängelrüssler, Gefleckten Kohltriebrüssler und Rapsglanzkäfer. - Raps 1, 10-14.

LERIN, J. (1988): Pertes de rendement associées à 2 ravageurs successifs (Ceutorrhnychus napi Gyll. et Meligethes aeneus F.) sur colza d'hiver (variété Bienvenu). - Agronomie 8, 251-256.

LERIN, J. (1995): Assessment of yield losses caused by insects in winter oilseed rape, a critical review. - IOBC wprs Bull. 95-101.

MAKOWSKI, N. (1995): Raps reagiert stark auf die Aussaat. - Hannoversche Land- und Forstwirtschaftliche Zeitung 32, 7-8.

MCGREGOR, D. I. (1987): Effect of plant density on development and yield of rapeseed and its significance to recovery from hail injury. - Can. J. Plant Sci. 67, 43-51.

Mendham, N. J. \& ScotT, R. K. (1975): The limiting effect of plant size at inflorescence initiation on subsequent growth and yield of oilseed rape (Brassica napus). - J. agric. Sci. Camb. 84, 487-502.

Meuche, A. (1940): Untersuchungen am Rapserdfloh (Psylliodes chrysocephala L.) in Ostholstein. - Z. angew. Entomol. 27, 464-492.

Meuche, A. (1942): Zur Ökologie und Bekämpfung des Großen Rapsstengelrüßlers (Ceutorrhynchus napi Gyll.). - Z. PflKrankh. (Pflanzenpath.) PflSchutz 52, 1-29.

Meuche, A. (1944): Zur Überwinterung des Rapserdflohs (Psylliodes chrysocephala L.). - Z. PflKrankh. (Pflanzenpath.) PflSchutz 54, 138-153.

Mohr, K.-H. (1960): Erdflöhe (Col. Chrys. Halticinae). - Die Neue Brehm-Bücherei, A. Ziemsen Verlag, Wittenberg Lutherstadt, 48 S.

Mомон, E. J. J. \& ZHOU, W. (2001): Growth and yield responses to plant density and stage of transplanting in winter oilseed rape (Brassica napus L.). - J. Agron. Crop Sci. 186, 253 $-259$.

Morice, M. (1960): La sélection du colza d'hiver basée sur l'étude des composantes du rendete-ment. - Ann. amélior. plant. 10, 85-116 (deutsche Zusammenfassung).

MüHLEnBERG, M. (1993): Freilandökologie. - 3. überarb. Aufl., Verlag Quelle \& Meyer, Heidelberg, Wiesbaden:, $512 \mathrm{~S}$.

NiLSSON, C. (2002): Strategies for the control of cabbage stem flea beetle on winter rape in Sweden. - IOBC/WPRS Bull. 25, 133-139.

NisSEN, U. (1997): Ökologische Studien zum Auftreten von Schadinsekten und ihren Parasitoiden an Winterraps norddeutscher Anbaugebiete. - Diss. Univ. Kiel, $141 \mathrm{~S}$. 
NitzSCHE, O. (1998): Auftreten und Effizienz von Parasitoiden als natürliche Gegenspieler von Schadinsekten im Winterraps unter besonderer Berücksichtigung unterschiedlicher Bodenbearbeitungsmaßnahmen nach Winterraps. - Diss. Univ. Göttingen, 166 S.

Nolte, H. W. (1953): Beiträge zur Epidemiologie und Prognose des Rapserdflohs (Psylliodes chrysocephala L.). - Beitr. Ent. 3, 518-529.

OpPERMANN, C. (1990): Untersuchungen zum Auftreten des Rapsstengelrüßlers (Ceutorhynchus napi Gyllenhal) am Winterraps (Brassica napus L. var. oleifera Metzg.) und Möglichkeiten der Überwachung und Bekämpfung. - Diss. Univ. Rostock, 100 S.

PAlosz, F. (1980): Einschätzung der Schadenskoeffizienten einiger Rapsschädlinge. - Tag.Ber. Akad. Landwirtsch.-Wiss. DDR 181, 135-143.

PAul, V. H. (2003): Raps - Krankheiten, Schädlinge, Schadpflanzen. - 3. überarb. Aufl., Th. Mann Verlag, Gelsenkirchen-Buer, 200 S.

PIMENTEL, D. (1961): The influence of plant spatial patterns on insect populations. - Ann. Entomol. Soc. Am. 54, 61-69.

READER, R. J. (1992): Herbivory as a confounding factor in an experiment measuring competition among plants. - Ecology 73, 373-376.

Root, R. B. (1973): Organisation of a plant-arthropod association in simple and diverse habitats: the fauna of collards, Brassica oleracea - Ecol. Monogr. 43, 95-124.

RoOT, R. B. \& KAREIVA, P. M. (1984): The search for resources by cabbage butterflies (Pieris rapae): Ecological consequences and adaptive significance of Markovian movements in a patchy environment. - Ecology 65, 147-165.

SAUERMANN, W. (1997): Kann die Saatstärke von Hybridraps reduziert werden? - Raps 15, 122-124.

SAUERMANN, W. \& GRONOW, J. (1999): Optimale Saatstärke von Hybridraps. - Raps 17, $124-$ 127.

SAuERmanN, W. \& GRONOW, J. (2000): Anbautechnik Hybridraps. Ist eine sorten- und standortspezifische Intensität möglich? - Raps 18, 4-8.

SCARISBrick, D. H., DANiEls, R. W. \& NoOR RAWI, A. B. (1982): The effect of varying seed rate on the yield and yield components of oil-seed rape (Brassica napus). - J. Agric. Sci. Camb. 99, $561-568$.

SCHERF, H. (1964): Die Entwicklungsstadien der mitteleuropäischen Curculioniden (Morphologie, Bionomie, Ökologie). - Abh. senckenb. naturf. Ges. 506, 1-335.

SCHMUTTERER, H. (1956): Zur Lebensweise und Bekämpfung des Großen Rapsstengelrüßlers Ceuthorrhynchus napi (Gyll.). - Z. angew. Entomol. 39, 302-315. 
Schотт, H. (1962): Untersuchungen über den Massenwechsel des Rapserdflohes (Psylliodes chrysocephala L.) von 1955 bis 1958 in den Bezirken Dresden, Leipzig und KarlMarx-Stadt. - Nachrichtenbl. Dt. Pflanzenschutzdienst. (Berlin) NF 16, 61-65.

SCHRIMPF, D. (1954): Untersuchungen über den Blüten- und Schotenansatz bei Raps, Rübsen und Senf. - Z. f. Acker- und Pflanzenbau 97, 305-336.

SchröDter, H. \& Nolte, H.-W. (1954): Die Abhängigkeit der Aktivität des Rapserdflohs (Psylliodes chrysocephala L.) von klimatischen Faktoren, insbesondere Licht, Temperatur und Feuchtigkeit. - Beitr. Ent. 4, 528-543.

SchUlz, R.-R. (1983): Zur Biologie, Ökologie und Schadwirkung des Rapserdflohs (Psylliodes chrysocephala L.) am Winterraps (Brassica napus L. var. oleifera Metzg.). - Diss. Univ. Rostock, $147 \mathrm{~S}$.

SCHULZ, R.-R. (1985): Untersuchungen zur Vermehrungsrate des Rapserdflohs (Psylliodes chrysocephala L.). - Arch. Phytopathol. Pfl. 21, 305-311

SchUlz, R.-R. (1993): Biologie und Bekämpfung des Rapserdflohs. - Raps 11, 176-177.

Schulz, R.-R. (1994): Untersuchungen zum Einfluß von Aussaat und Witterung auf die Pflanzenentwicklung von Raps. - Rapssymposium zu Fragen der Phytopathologie und des Pflanzenschutzes, Th. Mann Verlag, Gelsenkirchen, 139-143.

Schulz, R.-R. \& DAebeler, F. (1984): Zum Schaden durch den Rapserdfloh (Psylliodes chrysocephala L.), insbesondere seiner Imagines. - Nachrichtenbl. Pflanzenschutz DDR 38, 113-115.

SchwerdTfEger, F. (1968): Demökologie. Struktur und Dynamik tierischer Populationen. Paul Parey Verlag, Hamburg-Berlin, 448 S.

SEIGNER, L. (1993): Untersuchungen zur Verbesserung der Fängigkeit von Gelbschalen. Ges. Pflanzen 45, 83-85.

Shea, K., Symth, M., Sheppard, A., Morton, R. \& Chalimbaud, J. (2000): Effect of patch size and plant density of Paterson's curse (Echium plantagineum) on the oviposition of a specialist weevil, Mogulones larvatus. - Oecologia 124, 615-621.

SIERTS, H.-P. (1987): Untersuchungen zum Einfluß der intraspezifischen Konkurrenz auf Ausprägung und Stabilität (Ökovalenz) der leistungsbestimmenden Merkmale bei Winterraps (Brassica napus L.) unter Berücksichtigung der Ertragsstruktur. - Diss. Univ. Kiel, $95 \mathrm{~S}$.

Sierts, H.-P., Geißler, G., LÉOn, J. \& DiePenBrock, W. (1987): Stability of Yield Components from Winter Oil-Seed Rape (Brassica napus L.). - J. Agron. Crop Sci. 158, 107113. 
SoKal, R. R. \& Rohlf, F. J. (1995): Biometry. - 3. Aufl., Verlag W. H. Freeman and Company, New York, 887 S.

Sol, R. (1971): Beitrag zur Frage der Eiablage der Brachfliege (Phorbia coarctata Fall.) auf Kulturflächen, künstlicher Brache und im Laboratorium sowie ihres Fluges im Freien. - Z. angew. Entomol 67, 397-411.

SPEYER, W. (1921): Beitrag zur Biologie des Gefleckten Kohltriebrüßlers (Ceutorrhynchus quadridens Panz. - Entomologische Blätter Berlin 17, 118-124.

Stauß, R., Bleiholder, H., van den Boom, T., Buhr, L., Hack, H., Heß, M., Klose, R., MEIER, U. \& WEBER, E. (1994): Einheitliche Codierung der phänologischen Entwicklungsstadien mono- und dikotyler Pflanzen. Erweiterte BBCH-Skala. - Ciba-Geigy AG, Basel.

SteInBACH, P. (1990): Untersuchungen zur Pathogenese und Bekämpfung der durch Leptosphaeria maculans (Desm.) Ces et de Not. (Pyknidienstadium: Phoma lingam (Tode ex Fr.) Desm.) verursachten Wurzelhals- und Stengelfäule an Winterraps (Brassica napus L. var. oleifera Metzger). - Diss. Univ. Rostock, 186 S.

StOY, A. (1981): Ertragsbildung bei Raps in Anhängigkeit von der Bestandesdichte. - Schriftenreihe Agrarwiss. 32, 61-67.

STOY, A. (1983): Untersuchungen zur Konkurrenz bei Winterraps (Brassica napus L. var. napus) vor und nach dem Überwintern und deren Bedeutung für das Ertragspotential des Bestandes. - Diss. Univ. Kiel, 97 S.

SUNDERLAND, K. D. (1987): A review of methods of quantifying invertebrate predation occurring in the field. - Acta Phytopathol. Entomol. Hung. 22, 13-34.

Trommer, R. (1991): Methoden zur Ermittlung der Populationsdichte tierischer Schädlinge mit Hilfe von gruppierten Stichproben, erläutert am Beispiel der Wintersaateule (Agrotis segetum (Schiff. et Den.)). - Arch. Phytopathol. Pflanzenschutz 27, 289-298.

UlBer, B. (1994): Untersuchungen zur Abundanzdynamik von Ceutorhynchus napi Gyll. und Ceutorhynchus pallidactylus (Mrsh.) im Raum Göttingen. - Rapssymposium zu Fragen der Phytopathologie und des Pflanzenschutzes, Th. Mann Verlag, Gelsenkirchen, 9598.

Vullioud, P. (1974): Etude de l'influence de la date de semis sur le developpment du colza d' automne. - 4. Intern. Rapskongress, Gießen, 4.-8. Juni, 153-171.

WAHMHOFF, W. (2000): Integrierter Rapsanbau: Untersuchungen zur Entwicklung integrierter Produktionsverfahren am Beispiel des Winterrapses (Brassica napus L.). - Erich Schmidt Verlag, Berlin, 284 S. 
WinfIELD, A. L. (1992): Management of oilseed rape pests in Europe. - Zool. Rev. 5, 51-95.

Williams, R. H. \& FiTT, B. D. L. (1999): Differentiating A and B groups of Leptosphaeria maculans, causal agent of stem canker (blackleg) of oilseed rape: a review. - Plant Pathol. 46, 161-175.

Williams, I. H., BÜCHI, R. \& UlBeR, B. (2003): 10. Sampling, trapping and rearing oilseed rape pests and their parasitoids. - In: ALFORD, D. V. (Hrsg.): Biocontrol of oilseed rape pests. - Blackwell Verlag, Berlin, 145-160. 


\section{Anhang}

Tab. A-1: Mittlere Larvendichte von $P$. chrysocephala in Pflanzen ( \pm SE) verschiedener Saatstärken im Versuch 1999/00 am Standort Weendelsbreite

\begin{tabular}{cccc}
\hline \multirow{2}{*}{ Saatstärke } & \multicolumn{3}{c}{11.11 .1999} \\
& Larven/ & Larven/ \\
& Pflanze & & Endknospe \\
& $\mathrm{X}_{\mathrm{m}} \pm \mathrm{SE}$ & & $\mathrm{X}_{\mathrm{m}} \pm \mathrm{SE}$ \\
\hline $30 \mathrm{Körner} / \mathrm{m}^{2}$ & $11,1 \pm 1,0$ & a & $1,6 \pm 0,2$ \\
$60 \mathrm{Körner} / \mathrm{m}^{2}$ & $8,5 \pm 1,4$ & ab & $1,5 \pm 0,2$ \\
$90 \mathrm{Körner} / \mathrm{m}^{2}$ & $6,6 \pm 0,8$ & $\mathrm{~b}$ & $1,3 \pm 0,3$ \\
\hline Verschiedene Buchstaben indizieren signifikante Unterschiede zwischen
\end{tabular}

den Saatstärken im jeweiligen Jahr, ANOVA, Tukey-Test, $p<0,05$

Tab. A-2: Mittlere Larvendichte/Pflanze von P. chrysocephala in Pflanzen ( \pm SE) verschiedener Saatstärken im Versuch 2000/01 am Standort Weendelsbreite

\begin{tabular}{|c|c|c|c|c|c|c|c|}
\hline Saatstärke & $\begin{array}{r}20.10 .00 \\
X_{m} \pm S E\end{array}$ & & $\begin{array}{r}31.10 .00 \\
X_{m} \pm S E\end{array}$ & & $\begin{array}{r}16.11 .00 \\
X_{m} \pm S E\end{array}$ & & $\begin{array}{r}15.02 .01 \\
X_{m} \pm S E\end{array}$ \\
\hline 30 Körner/m² & $7,6 \pm 0,7$ & $a$ & $13,7 \pm 0,9$ & $a$ & $16,4 \pm 1,4$ & $a$ & $18,9 \pm 2,0$ \\
\hline 60 Körner/m² & $4,3 \pm 0,4$ & $b$ & $7,9 \pm 1,2$ & $b$ & $10,3 \pm 1,1$ & $b$ & $9,6 \pm 1,3$ \\
\hline 90 Körner/m² & $2,4 \pm 0,2$ & $\mathrm{C}$ & $4,7 \pm 0,7$ & $\mathrm{C}$ & $6,4 \pm 0,5$ & $b$ & $6,5 \pm 0,5$ \\
\hline
\end{tabular}

Verschiedene Buchstaben indizieren signifikante Unterschiede zwischen den Saatstärken im jeweiligen Jahr, ANOVA, Tukey-Test, 2000: $p<0,05,2001: p<0,01$

Tab. A-3: Mittlere Larvendichte/Pflanze von $P$. chrysocephala in Pflanzen ( \pm SE) verschiedener Saatstärken im Versuch 2001/02 am Standort Weendelsbreite

\begin{tabular}{|c|c|c|c|c|c|}
\hline Saatstärke & $\begin{array}{r}12.11 .01 \\
\mathrm{X}_{\mathrm{m}} \pm \mathrm{SE} \\
\end{array}$ & & $\begin{array}{r}29.11 .01 \\
X_{m} \pm S E \\
\end{array}$ & & $\begin{array}{r}31.01 .02 \\
X_{m} \pm S E \\
\end{array}$ \\
\hline 30 Körner $/ \mathrm{m}^{2}$ & $7,9 \pm 1,0$ & $d$ & $11,9 \pm 1,4$ & $a$ & $15,2 \pm 1,6 \quad a$ \\
\hline 60 Körner/m² & $5,5 \pm 0,8$ & a & $8,1 \pm 0,7$ & b & $13,3 \pm 1,5$ a \\
\hline
\end{tabular}

Verschiedene Buchstaben stellen signifikante Unterschiede zwischen den Saatstärken im jeweiligen Jahr dar, 2001: ANOVA, Tukey-Test, $p<0,05$, 2002: Mann-Whitney U-Test, $p<$ 0,01 
Tab. A-4: Mittlere Dichte der Eigelege und Larven von C. napi in den Haupt- und Seitentrieben der Pflanzen $( \pm \mathrm{SE})$ verschiedener Saatstärken in den Monaten April bzw. Mai der Jahre 2000 und 2001 am Standort Weendelsbreite und des Jahres 2002 am Standort Otto-Hahn-Straße

\begin{tabular}{|c|c|c|c|c|c|c|c|c|}
\hline \multirow[t]{2}{*}{ Jahr } & \multirow[t]{2}{*}{ Saatstärke } & \multicolumn{4}{|c|}{ Eiablagestellen } & \multicolumn{3}{|c|}{ Larven } \\
\hline & & $\begin{array}{l}\text { Haupttrieb } \\
X_{m} \pm S E\end{array}$ & & $\begin{array}{l}\text { Seitentrieb } \\
X_{m} \pm S E\end{array}$ & & $\begin{array}{l}\text { Haupttrieb } \\
X_{m} \pm S E\end{array}$ & & $\begin{array}{l}\text { Seitentrieb } \\
X_{m} \pm S E\end{array}$ \\
\hline \multirow[t]{3}{*}{2000} & 30 Körner/m² & $1,4 \pm 0,2$ & $a$ & $0,2 \pm 0,1$ & $\bar{a}$ & $0,8 \pm 0,2$ & $a$ & $0,2 \pm 0,1$ \\
\hline & 60 Körner/m² & $1,1 \pm 0,2$ & $a$ & $0,0 \pm 0,0$ & a & $0,7 \pm 0,2$ & $a$ & $0,1 \pm 0,0$ \\
\hline & 90 Körner/m² & $0,9 \pm 0,2$ & $a$ & $0,0 \pm 0,0$ & a & $0,7 \pm 0,2$ & $a$ & $0,0 \pm 0,0$ \\
\hline \multirow[t]{3}{*}{2001} & 30 Körner/m² & $1,8 \pm 0,4$ & $a$ & $0,4 \pm 0,1$ & $a$ & $1,2 \pm 0,4$ & $a$ & $0,9 \pm 0,3$ \\
\hline & 60 Körner/m² & $1,0 \pm 0,1$ & $a b$ & $0,1 \pm 0,1$ & $a$ & $0,9 \pm 0,2$ & $a$ & $0,2 \pm 0,1 a$ \\
\hline & 90 Körner/m² & $0,6 \pm 0,2$ & $\mathrm{~b}$ & $0,1 \pm 0,0$ & $a$ & $0,5 \pm 0,2$ & $a$ & $0,0 \pm 0,0$ \\
\hline \multirow[t]{2}{*}{2002} & 30 Körner/m² & $7,4 \pm 1,2$ & $a$ & $1,0 \pm 0,2$ & $a$ & $3,1 \pm 0,8$ & $a$ & $2,6 \pm 1,9$ \\
\hline & 60 Körner/m² & $6,1 \pm 1,0$ & $a$ & $0,2 \pm 0,1$ & $b$ & $3,4 \pm 0,4$ & $a$ & $0,1 \pm 0,1$ \\
\hline
\end{tabular}

Unterschiedliche Buchstaben indizieren signifikante Unterschiede zwischen den Saatstärken im jeweiligen Jahr, 2000 und 2001: ANOVA, Tukey-Test, 2002: Mann-Whitney U-Test, $p<0,01$

Tab. A-5: Mittlere Dichte der Eigelege und Larven von C. pallidactylus in den Haupt- und Seitentrieben der Pflanzen $( \pm \mathrm{SE})$ verschiedener Saatstärken in den Monaten April bzw. Mai der Jahre 2000 und 2001 am Standort Weendelsbreite und des Jahres 2002 am Standort Otto-Hahn-Straße

\begin{tabular}{|c|c|c|c|c|c|c|c|}
\hline \multirow[t]{3}{*}{ Jahr } & \multirow[t]{3}{*}{ Saatstärke } & \multicolumn{4}{|c|}{ Eiablagestellen } & \multicolumn{2}{|c|}{ Larven } \\
\hline & & $\begin{array}{l}\text { Blätter am } \\
\text { Haupttrieb }\end{array}$ & $\begin{array}{l}\text { Blätter am } \\
\text { Seitentrieb }\end{array}$ & Haupttrieb & Seitentrieb & Haupttrieb & Seitentrieb \\
\hline & & $X_{m} \pm S E$ & $X_{m} \pm S E$ & $X_{m} \pm S E$ & $\mathrm{X}_{\mathrm{m}} \pm \mathrm{SE}$ & $X_{m} \pm S E$ & $\mathrm{X}_{\mathrm{m}} \pm \mathrm{SE}$ \\
\hline \multirow[t]{3}{*}{2000} & 30 Körner/m² & $1,3 \pm 0,2 a$ & $0,2 \pm 0,1 \mathrm{a}$ & $0,0 \pm 0,0 \mathrm{a}$ & $0,0 \pm 0,0 a$ & $8,5 \pm 1,1 \mathrm{a}$ & $2,5 \pm 0,5 a$ \\
\hline & 60 Körner/m² & $0,7 \pm 0,1 b$ & $0,0 \pm 0,0 \mathrm{~b}$ & $0,1 \pm 0,0 b$ & $0,0 \pm 0,0 a$ & $5,5 \pm 0,8 a b$ & $1,0 \pm 0,4 b$ \\
\hline & 90 Körner/m² & $0,5=$ & $0,0 \pm$ & $0,1 \pm 0,1 b$ & $0,0 \pm 0,0 a$ & $4,6 \pm 0,5 b$ & $0,1 \mathrm{~b}$ \\
\hline \multirow[t]{3}{*}{2001} & 30 Körner/m² & $0,5 \pm 0,2 \mathrm{a}$ & $0,0 \pm 0,0 \mathrm{a}$ & $0,0 \pm 0,0 a$ & $0,0 \pm 0,0 a$ & $2,5 \pm 0,6 a$ & $3,4 \pm 0,5 a$ \\
\hline & 60 Körner/m² & $0,3 \pm 0,0 \mathrm{a}$ & $0,0 \pm 0,0 \mathrm{a}$ & $0,0 \pm 0,0 \mathrm{a}$ & $0,0 \pm 0,0 \mathrm{a}$ & $3,2 \pm 0,8 a$ & $1,1 \pm 0,3 b$ \\
\hline & 90 Körner/m² & $0,1 \pm 0,0 \mathrm{a}$ & $0,0 \pm 0,0 a$ & $0,0 \pm 0,0 a$ & $0,0 \pm 0,0 a$ & $1,6 \pm 0,3 a$ & $0,3 \pm 0,1 b$ \\
\hline \multirow[t]{2}{*}{2002} & 30 Körner/m² & $4,5 \pm 1,0 \mathrm{a}$ & $0,0 \pm 0,0 \mathrm{a}$ & $0,1 \pm 0,1 \mathrm{a}$ & $0,1 \pm 0,0 \mathrm{a}$ & $5,0 \pm 1,0 a$ & $6,3 \pm 2,4 a$ \\
\hline & 60 Körner/m² & $2,0 \pm 0,3 b$ & $0,0 \pm 0,0 \mathrm{a}$ & $0,0 \pm 0,0 a$ & $0,0 \pm 0,0 \mathrm{a}$ & $3,1 \pm 0,3 a$ & $1,5 \pm 0,4 a$ \\
\hline
\end{tabular}

Unterschiedliche Buchstaben indizieren signifikante Unterschiede zwischen den Saatstärken im jeweiligen Jahr, 2000 und 2001: ANOVA, Tukey-Test, 2002: Mann-Whitney U-Test, $p<0,01$ 
Tab. A-6: Mittlere Fangsumme der Imagines in Gelbschalen und mittlere Larvenabundanzen von C. napi und C. pallidactylus in den Jahren 2000 bis 2002

\begin{tabular}{ccccc}
\hline Versuchsjahr & \multicolumn{2}{c}{ C. napi } & \multicolumn{2}{c}{ C. pallidactylus } \\
& $\begin{array}{c}\text { Mittlere Summe } \\
\text { Käfer/Gelbschale } \\
\text { (bis BBCH 50) }\end{array}$ & $\begin{array}{c}\text { Mittlere Anzahl } \\
\text { Larven/m² }\end{array}$ & $\begin{array}{c}\text { Mittlere Summe } \\
\text { Käfer/Gelbschale } \\
\text { (bis BBCH 50) }\end{array}$ & $\begin{array}{c}\text { Mittlere Anzahl } \\
\text { Larven/m² }\end{array}$ \\
\hline 2000 & 10,0 & 50,3 & 16,0 & 413,6 \\
2001 & 38,5 & 49,9 & 56,0 & 168,4 \\
2002 & 35,4 & 152,5 & 34,5 & 252,7 \\
\hline
\end{tabular}

Tab. A-7: Mittlere Eidichte $( \pm c v[\%])$ von $P$. chrysocephala innerhalb des Wurzelbereiches der Pflanze und an pflanzenfreien Stellen (Eier/255 $\left.\mathrm{cm}^{2}\right)$ in Parzellen mit unterschiedlichen Saatstärken am Standort Weendelsbreite im Versuch 2000

\begin{tabular}{ccc}
\hline Saatstärke & \multicolumn{2}{c}{ Eier/255 $\mathrm{cm}^{2}$} \\
& Wurzelbereich & pflanzenfreie Stellen \\
& $X_{m} \pm c v[\%]$ & $X_{m} \pm c v[\%]$ \\
\hline $30 \mathrm{Körner} / \mathrm{m}^{2}$ & $5,2 \pm 11,5$ & $1,6 \pm 25$ \\
$60 \mathrm{Körner} / \mathrm{m}^{2}$ & $5,3 \pm 26,3$ & - \\
$90 \mathrm{Körner} / \mathrm{m}^{2}$ & $3,9 \pm 28,4$ & - \\
\hline
\end{tabular}

Tab. A-8: Mittlere Eidichte ( $\pm c v[\%])$ von $P$. chrysocephala bei verschiedenen Entfernungsstufen $\left(\right.$ Eier $\left./ 90 \mathrm{~cm}^{2}\right)$ und pro $\mathrm{m}^{2}$ in Parzellen mit unterschiedlichen Saatstärken am Standort Otto-Hahn-Straße im Versuch 2001

\begin{tabular}{lcccr}
\hline Saatstärke & \multicolumn{5}{c}{ Eier $/ 90 \mathrm{~cm}^{2}$} & Eier $/ \mathrm{m}^{2}$ \\
& $0-5 \mathrm{~cm}$ & $5-10 \mathrm{~cm}$ & $10-15 \mathrm{~cm}$ & \\
& $\mathrm{X}_{\mathrm{m}} \pm \mathrm{CV}[\%]$ & $\mathrm{X}_{\mathrm{m}} \pm \mathrm{CV}[\%]$ & $\mathrm{X}_{\mathrm{m}} \pm \mathrm{CV}[\%]$ & $\mathrm{X}_{\mathrm{m}} \pm \mathrm{CV}[\%]$ \\
\hline $30 \mathrm{Körner} / \mathrm{m}^{2}$ & $3,8 \pm 18,4$ & $1,5 \pm 60,7$ & $1,3 \pm 36,8$ & $66,7 \pm 49,8$ \\
$60 \mathrm{Körner} / \mathrm{m}^{2}$ & $4,1 \pm 19,1$ & $2,8 \pm 6,7$ & $2,1 \pm 38,1$ & $99,7 \pm 55,7$ \\
\hline
\end{tabular}




\section{Danksagung}

Herrn Prof. Dr. Stefan Vidal danke ich für die Übernahme des Referats, für die Stellung des Themas in Zusammenarbeit mit Herrn Dr. Bernd Ulber, die wertvollen Ratschläge, die Bereitstellung eines Arbeitsplatzes sowie die Ermöglichung der Feldversuche.

Prof. Dr. Andreas von Tiedemann danke ich für die Übernahme des Korreferates.

Mein besonderer Dank gilt Herrn Dr. Bernd Ulber für seine zahlreichen Anregungen und die stete Diskussions- und Hilfsbereitschaft.

Herrn Dr. Udo Heimbach und Herrn Dr. Ralf-Rainer Schulz danke ich für ihre jederzeit motivierende und kritische Diskussionsbereitschaft.

Frau Katharina Lohaus, Frau Ines Mosebach, Frau Julia Morzfeld und Frau Yvonne Seidel danke für ihre äußerst gewissenhafte und engagierte Unterstützung bei der Erledigung der Feld- und Laborarbeiten.

Allen Mitarbeiterinnen und Mitarbeitern der entomologischen Abteilung danke ich für eine angenehm-konstruktive Arbeitsatmosphäre und die spontane Hilfe bei der Bewältigung verschiedener Probleme.

Vor allem danke ich meinem Mann und meinen Eltern, die mich in allen Phasen meiner Arbeit durch viel Geduld und entspannende Ablenkung unterstützt haben.

Die Arbeit wurde aus Mitteln des DFG-Graduiertenkollegs „Landwirtschaft und Umwelt“ gefördert. 


\section{Lebenslauf}

Persönliche Daten

Helene Nuss

geboren am 13.12.1970 in Leninabad/Tadschikistan

Schulausbildung

09/1978 - 08/1988

Mittelschule in Leninabad, Realschulabschluss: Abitur (SU)

$03 / 1991-02 / 1993$

Sonderlehrgang beim Eichsfeldgymnasium Duderstadt

Abitur im Februar 1993

\section{Hochschulausbildung}

$09 / 1988-06 / 1989$

Polytechnische Hochschule in Tomsk, Fachbereich: Robotertechnische Systeme

Abbruch wegen der Ausreise

$10 / 1993-05 / 1999$

Studium der Biologie an der TU Braunschweig

$09 / 1999-07 / 2004$

Promotion im Fachbereich Agrarwissenschaften der GeorgAugust-Universität Göttingen

\section{Berufliche Tätigkeiten}

04/1999-08/1999

Wissenschaftliche Mitarbeiterin am Institut für Pflanzenschutz in Ackerbau und Grünland der Biologischen Bundesanstalt Braunschweig

$01 / 2002-12 / 2002$

Wissenschaftliche Mitarbeiterin am Institut für Pflanzenpathologie und Pflanzenschutz der Georg-August-Universität Göttingen 